\title{
Site-Selective Catalytic Deaminative Alkylation of Unactivated Olefins
}

\author{
Shang-Zheng Sun, ${ }^{\dagger}$ Ciro Romano ${ }^{\dagger}$ and Ruben Martin ${ }^{\dagger \S *}$ \\ rmartinromo@iciq.es \\ $\dagger$ Institute of Chemical Research of Catalonia (ICIQ), The Barcelona Institute of Science and \\ Technology, Av. Països Catalans 16, 43007 Tarragona, Spain \\ $\S$ ICREA, Passeig Lluís Companys, 23, 08010, Barcelona, Spain
}

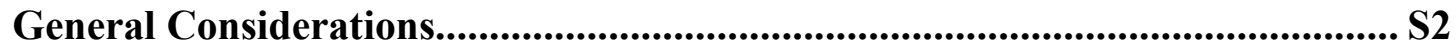

Optimization of the Reaction Conditions .............................................................. S3

Synthesis of Ligands and Starting Materials ......................................................... S6

Ni-Catalyzed Deaminative Alkylation with Terminal Olefins............................ S113

Ni-Catalyzed Deaminative Alkylation with Internal Olefins................................ S25

Large Scale Reaction and Synthetic Applications.............................................. S404

Mechanistic Experiments.................................................................................... S47

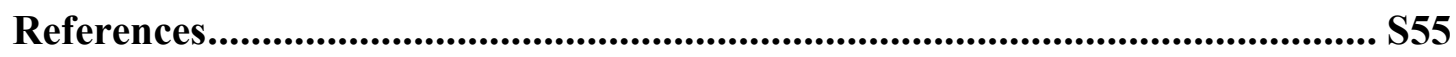

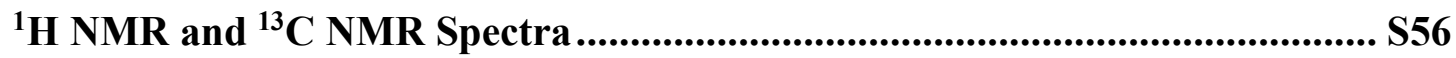




\section{General Considerations}

Reagents. Commercially available materials were used as received without further purification. $\mathrm{NiI}_{2}$ and $\mathrm{NiBr}_{2} \cdot \mathrm{DME}$ (97\% purity) were purchased from Aldrich, $(\mathrm{EtO})_{2} \mathrm{MeSiH}$ (DEMS, 97\% purity) and (EtO) $)_{3} \mathrm{SiH}$ were purchased from Fluorochem and Alfa Aesar. Sodium phosphate dibasic $\left(\mathrm{Na}_{2} \mathrm{HPO}_{4}\right)$ was purchased from Scharlau. Anhydrous $N, N$-Dimethylacetamide (NMP, 99.5\% purity), Anhydrous 1,4-dioxane (99.5\% purity) and Dimethyl sulfoxide (DMSO, 99.5 purity) were purchased from Acros.

Analytical methods. ${ }^{1} \mathrm{H}$ and ${ }^{13} \mathrm{C}$ NMR spectra were recorded on Bruker $300 \mathrm{MHz}$, Bruker $400 \mathrm{MHz}$ and Bruker $500 \mathrm{MHz}$ at $20^{\circ} \mathrm{C}$. All ${ }^{1} \mathrm{H}$ NMR spectra are reported in parts per million (ppm) downfield of TMS and were calibrated using the residual solvent peak of $\mathrm{CHCl}_{3}\left(7.26 \mathrm{ppm}\right.$ ), unless otherwise indicated. All ${ }^{13} \mathrm{C}$ NMR spectra are reported in ppm relative to TMS, were calibrated using the signal of residual $\mathrm{CHCl}_{3}(77.16 \mathrm{ppm}),{ }^{11} \mathrm{~B} \mathrm{NMR}$ and ${ }^{19} \mathrm{~F}$ NMR were obtained with ${ }^{1} \mathrm{H}$ decoupling unless otherwise indicated. Coupling constants, $J$, are reported in Hertz. Melting points were measured using open glass capillaries in a Büchi B540 apparatus. Gas chromatographic analyses were performed on Hewlett-Packard 6890 gas chromatography instrument with FID detector. Flash chromatography was performed with EM Science silica gel 60 (230-400 mesh). Thin layer chromatography was used to monitor reaction progress and analyze fractions from column chromatography. To this purpose TLC Silica gel $60 \mathrm{~F}_{254}$ aluminium sheets from Merck were used and visualization was achieved using UV irradiation and/or staining with $\mathrm{KMnO}_{4}$. The yields reported in Table 2-3 and scheme 2 refer to isolated yields and represent an average of at least two independent runs. The procedures described in this section are representative. Thus, the yields may differ slightly from those given in the Schemes of the manuscript. In the cases the High-Resolution Mass Spectra of the molecular ion could not be obtained using ESI and APCI ionization modes the GC-MS of the compound was given. 


\section{Optimization of the Reaction Conditions}

General procedure: An oven-dried $8 \mathrm{~mL}$ screw-cap test tube containing a stirring bar was charged with $\mathrm{NiBr}_{2} \cdot \mathrm{DME}$ (10 mol\%), the corresponding ligand (15 mol\%), $\mathrm{Na}_{2} \mathrm{HPO}_{4}(0.4$ mmol, $56.8 \mathrm{mg}$ ) and 1-(1-(tert-butoxycarbonyl)piperidin-4-yl)-2,4,6-triphenylpyridin-1ium tetrafluoroborate (1a, $113.6 \mathrm{mg}, 0.2 \mathrm{mmol}$ ). Subsequently, the tube was sealed with a Teflon-lined screw cap, then evacuated and back-filled with Ar (3 times). Afterwards, 4phenyl-1-butene (2a, $90 \mu \mathrm{l}, 0.6 \mathrm{mmol}, 3.0$ equiv), (EtO) ${ }_{2} \mathrm{MeSiH}$ (DEMS, $96 \mu \mathrm{l}, 0.60 \mathrm{mmol}$, 3.0 equiv), NMP and 1.4-dioxane (4:1, 1.0 mL) were added via syringe. Then, the tube was stirred at $40{ }^{\circ} \mathrm{C}$ for $24 \mathrm{~h}$. After the reaction was completed, the mixture was diluted with EtOAc, filtered through silica gel and concentrated under vacuum. The yields were determined by GC FID analysis using 1-decane as internal standard, and the product was purified by column chromatography on silica gel (Hexane/ EtOAc 120:1 to 60:1). 
Table S1. Optimization of the Deaminative Alkylation with Terminal Olefins ${ }^{a, b}$

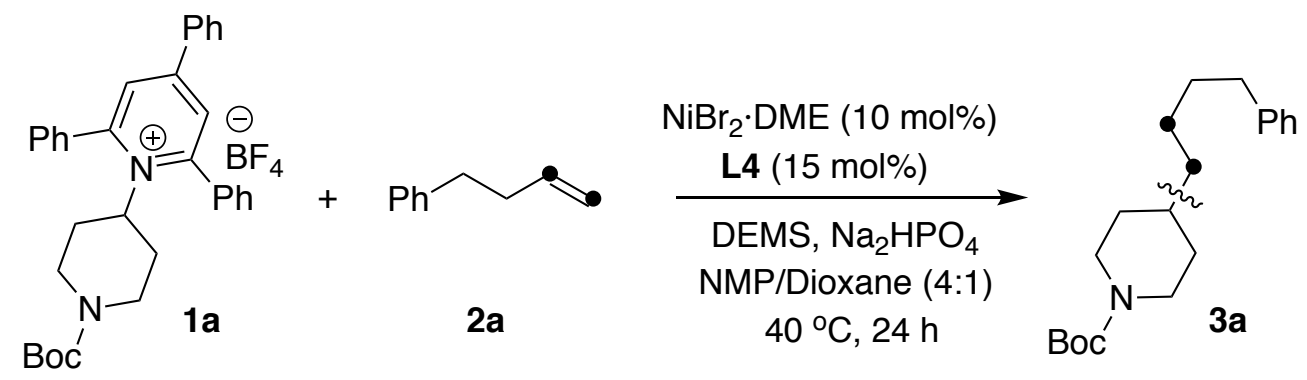

\begin{tabular}{|c|c|c|c|}
\hline Entry & Deviation standard conditions & $3 \mathbf{a}(\%)$ & \\
\hline 1 & $\mathrm{NiBr}_{2} \cdot \mathrm{DME}$ & $81(80)^{c}$ & \\
\hline 2 & Using $\mathrm{Nil}_{2}$ & trace & \\
\hline 3 & Using $\mathrm{NiBr}_{2}$ & trace & \\
\hline 4 & Using $\mathrm{NiBr}_{2} \cdot$ diglyme & 70 & $R^{1}$ \\
\hline 5 & Using $\mathrm{NiBr}_{2} \cdot(\mathrm{bpy})$ & 40 & $\mathrm{R}^{1}=\mathrm{H} ; \mathrm{R}^{2}=\mathrm{OMe}(\mathrm{L} \mathbf{1})$ \\
\hline 6 & Using $\mathrm{Ni}(\mathrm{COD})_{2}$ & 33 & $\mathrm{R}^{1}=\mathrm{H} ; \mathrm{R}^{2}=\mathrm{CF}_{3}(\mathrm{~L} 2)$ \\
\hline 7 & L1 instead of L4 & 66 & $\mathrm{R}^{1}=\mathrm{R}^{2}=\mathrm{H}(\mathrm{L} 3)$ \\
\hline 8 & L2 instead of L4 & 52 & $\mathrm{R}^{1}=\mathrm{H} ; \mathrm{R}^{2}=t-\mathrm{Bu}(\mathrm{L} 4)$ \\
\hline 9 & L3 instead of L4 & 58 & $\mathrm{R}^{1}=\mathrm{Me} ; \mathrm{R}^{2}=t-\mathrm{Bu}(\mathrm{L} 5)$ \\
\hline 10 & L5 instead of L4 & 3 & \\
\hline 11 & L6 instead of L4 & 11 & \\
\hline 12 & L7 instead of L4 & trace & \\
\hline 13 & L8 instead of L4 & 52 & 6 \\
\hline 14 & Using $(\mathrm{MeO})_{3} \mathrm{SiH}$ & 63 & \\
\hline 15 & Using $(\mathrm{EtO})_{3} \mathrm{SiH}$ & 32 & \\
\hline 16 & Using PMHS & 38 & Mé \\
\hline 17 & Using NMP & 51 & \\
\hline 18 & Using DMA & 42 & \\
\hline 19 & Using DMSO & 41 & \\
\hline 20 & Using 1,4-Dioxane & 6 & \\
\hline 21 & at $30^{\circ} \mathrm{C}$ & 54 & L8 \\
\hline 22 & no $\mathrm{Ni}, \mathrm{L} 4, \mathrm{Na}_{2} \mathrm{HPO}_{4}$ or DEMS & 0 & \\
\hline
\end{tabular}

${ }^{a}$ Conditions: 1a $\left(0.20 \mathrm{mmol}, 1.0\right.$ equiv), 2a (0.60 mmol, 3.0 equiv), $\mathrm{NiBr}_{2} \cdot \mathrm{DME}(10$ mol\%), L4 (15 mol\%), DEMS (0.60 mmol, 3.0 equiv), $\mathrm{Na}_{2} \mathrm{HPO}_{4}(0.40 \mathrm{mmol}, 2.0$ equiv), NMP/1,4-dioxane (4:1, $1.0 \mathrm{~mL}), 40{ }^{\circ} \mathrm{C}, 24 \mathrm{~h} .{ }^{\mathrm{b}}$ Yields were determined by GC FID, using 1-decane as the internal standard. DEMS $=$ Diethoxymethylsilane $\left((\mathrm{EtO})_{2} \mathrm{MeSiH}\right)$. PMHS = Polymethylhydrosiloxane. 
Table S2. Optimization of the Deaminative Alkylation with Internal Olefins ${ }^{a, b}$

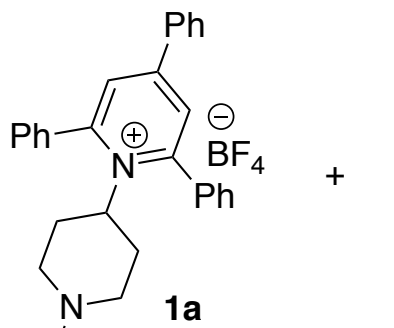

Boc'
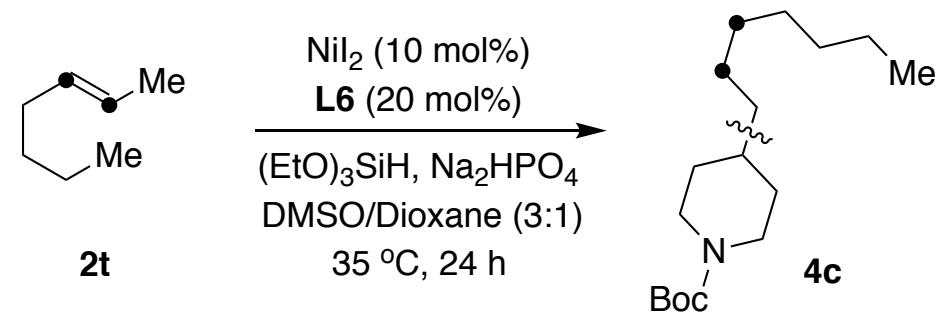

\section{Entry}

1

2

3

4

5

6

7

8

9

10

11

12

13

14

15

16

17

18
Deviation standard conditions

$\mathrm{Nil}_{2}$

Using $\mathrm{NiBr}_{2}$

Using $\mathrm{NiBr}_{2} \cdot \mathrm{DME}$

L5 instead of L6

L7 instead of L6

L9 instead of L6

L10 instead of 6

Using (EtO) $)_{2} \mathrm{MeSiH}$

Using $(\mathrm{MeO})_{3} \mathrm{SiH}$

Using $\mathrm{Et}_{3} \mathrm{SiH}$

Using PMHS

Using NMP

Using DMF

Using DMSO

Using 1,4-Dioxane

Using $\mathrm{KHCO} 3$

at $30^{\circ} \mathrm{C}$

no Ni, L6, $\mathrm{Na}_{2} \mathrm{HPO}_{4}$ or $(\mathrm{EtO})_{3} \mathrm{SiH}$

\section{4c $(\%)$}

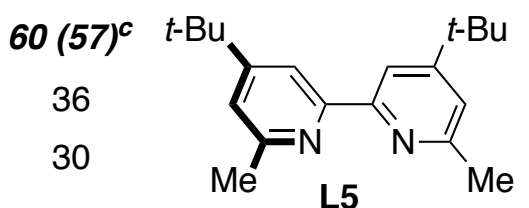

8

30

11

trace

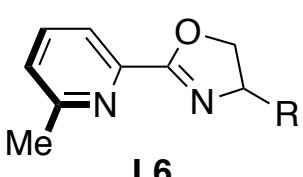

42

43

0

48

30

24

51

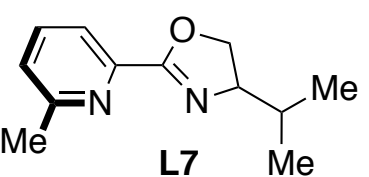

L7

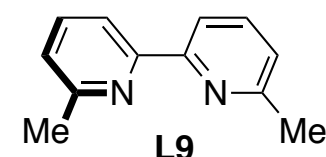

9

41

34

0

${ }^{a}$ Conditions: 1a (0.20 mmol, 1.0 equiv), $2 t$ ( $0.60 \mathrm{mmol}, 3.0$ equiv), $\mathrm{NiI}_{2}(10 \mathrm{~mol} \%), \mathbf{L 6}$ (20 mol\%), (EtO) $)_{3} \mathrm{SiH}$ (0.40 mmol, 2.0 equiv), $\mathrm{Na}_{2} \mathrm{HPO}_{4}(0.80 \mathrm{mmol}, 4.0$ equiv), DMSO/1,4-dioxane (3:1, $1.2 \mathrm{~mL}), 35{ }^{\circ} \mathrm{C}, 24 \mathrm{~h} .{ }^{\mathrm{b}}$ Yields were determined by GC FID, using 1-decane as the internal standard. PMHS = Polymethylhydrosiloxane. 


\section{Synthesis of Ligands and Starting Materials}

Commercially available compounds were used as received without further purification. 2-(6-methylpyridin-2-yl)-4,5-dihydrooxazole (L6), ${ }^{1}$ 4-isopropyl-2-(6-methylpyridin2-yl)-4,5-dihydrooxazole (L7), ${ }^{1} \quad$ 1-(1-(tert-butoxycarbonyl)piperidin-4-yl)-2,4,6triphenylpyridin-1-ium tetrafluoroborate (1a), ${ }^{2}$ 1-cyclohexyl-2,4,6-triphenylpyridin-1ium tetrafluoroborate (1b), ${ }^{2}$ 1-(4,4-difluorocyclohexyl)-2,4,6-triphenylpyridin-1-ium tetrafluoroborate (1c), ${ }^{2}$ 2,4,6-triphenyl-1-(tetrahydro-2H-pyran-4-yl)pyridin-1-ium tetrafluoroborate (1e), ${ }^{2} \quad 1$-(1-(tert-butoxycarbonyl)pyrrolidin-3-yl)-2,4,6triphenylpyridin-1-ium tetrafluoroborate (1f), ${ }^{3}$ 2,4,6-triphenyl-1-(4-phenylbutan-2yl)pyridin-1-ium tetrafluoroborate (1g), ${ }^{2}$ 1-(1-methoxy-4-methyl-1-oxopentan-2-yl)2,4,6-triphenylpyridin-1-ium tetrafluoroborate $\quad(\mathbf{1 j}),{ }^{4} \quad 1-(1-(2,6-$ dimethylphenoxy)propan-2-yl)-2,4,6-triphenylpyridin-1-ium tetrafluoroborate $(\mathbf{1 k}){ }^{2}$ (S)-1-(1-methoxy-1-oxopropan-2-yl)-2,4,6-triphenylpyridin-1-ium tetrafluoroborate (1n),$^{5}$ ((allyloxy)methyl)benzene (2b) ${ }^{6}$ tert-butyl(hex-5-en-1-yloxy)dimethylsilane (2c) ${ }^{7} \quad$ Hex-5-en-1-yl 4-fluorobenzoate $\quad(\mathbf{2 h}),{ }^{8} \quad$ (E)-tert-butyl(hex-4-en-1-yloxy) dimethylsilane (2u), ${ }^{9}$ 2-(oct-5-en-1-yl)isoindoline-1,3-dione (2x), ${ }^{10}$ 3-methyl-1-(oct-5en-1-yl)-1H-indole (2z), ${ }^{1} \quad$ tert-butyl 4-(prop-1-en-1-yl)piperidine-1-carboxylate (2aa),$^{11} \quad(8 R, 9 S, 13 S, 14 S)$-3-(hex-5-en-1-yloxy)-13-methyl-6, 7, 8,9,11,12,13,14,15,16decahydro-17H-cyclopenta $[a]$ phenanthrene-17-one (2ac), ${ }^{12}$ tert-butyl 2,5-dihydro1H-pyrrole-1-carboxylate (18), ${ }^{13}$ (1-vinylcyclopropyl)benzene $(\mathbf{2 1})^{14}$ were prepared by known procedures, the NMR data matched those reported previously in the literature.

\section{General Procedure for the Preparation of L5}

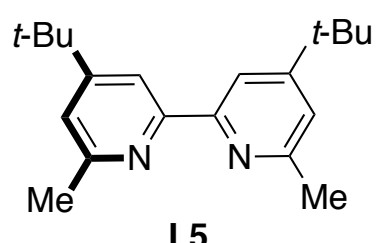

$\mathbf{L} 5$

4,4'-di-tert-butyl-6,6'-dimethyl-2,2'-bipyridine (L5). To a solution of 4,4'-di-tertbutyl-2,2'-bipyridine $(4.02 \mathrm{~g}, 15.0 \mathrm{mmol}, 1$ equiv. $)$ in THF $(75 \mathrm{~mL})$ at $0{ }^{\circ} \mathrm{C}$ under argon atmospheres was added a 1.6 M solution of $\mathrm{MeLi}$ in $\mathrm{Et}_{2} \mathrm{O}(47 \mathrm{~mL}, 75 \mathrm{mmol}, 5.0$ equiv.) and the mixture was stirred for $14 \mathrm{~h}$. The solution was then quenched water and extracted with $\mathrm{Et}_{2} \mathrm{O}$, dried over $\mathrm{MgSO}$, and concentrated under reduced pressure. The latter was then dissolved in $\mathrm{DCM}(50 \mathrm{~mL})$ and $\mathrm{MnO}_{2}$ (13 g, $150 \mathrm{mmol}, 10$ equiv.) was 
added and the mixture stirred at $60{ }^{\circ} \mathrm{C}$ for $4 \mathrm{~h}$. At this point the solution was filtered through a short pad of silica and concentrated under vacuum. The product was purified by flash chromatography (Hexane/EtOAc), obtained pale yellow solid $2.58 \mathrm{~g}$ (58\%). Mp 190-192 ${ }^{\circ} \mathrm{C} .{ }^{1} \mathbf{H}$ NMR $\left(400 \mathrm{MHz}, \mathrm{CDCl}_{3}\right): \delta 8.13(\mathrm{~d}, J=1.8 \mathrm{~Hz}, 2 \mathrm{H}), 7.14(\mathrm{~d}, J=$ $1.8 \mathrm{~Hz}, 2 \mathrm{H}), 2.63$ (s, 6H), 1.37 (s, 18H) ppm. ${ }^{13} \mathbf{C}$ NMR (101 MHz, $\left.\mathrm{CDCl}_{3}\right): \delta$ 161.0, 157.8, 156.7, 120.2, 115.8, 35.0, 30.8, 25.0 ppm. IR (neat, $\mathrm{cm}^{-1}$ ): 2959, 2903, 2867, 1591, 1557, 1460, 1390, 1290, 917, 863. HRMS calcd. for $\left(\mathrm{C}_{20} \mathrm{H}_{29} \mathrm{~N}_{2}\right)[\mathrm{M}+\mathrm{H}]^{+}$: 297.2325 found 297.2316 .

\section{General Procedure for the Preparation of Katritzky Pridinium Salts}<smiles>[R]C(N)N</smiles>

General Procedure: 2,4,6-triphenylpyrylium tetrafluoroborate (1.0 equiv) and an amine (1.2 equiv) were added to a Schlenk containing a stirring bar. This was followed by addition of dry EtOH $(1.0 \mathrm{M})$, resulting in a color change from yellow to black orange. The mixture was then stirred and heated at reflux in an oil bath at $90{ }^{\circ} \mathrm{C}$ for $5 \mathrm{~h}$. At that time, the mixture was allowed to cool to room temperature. $\mathrm{Et}_{2} \mathrm{O}$ was then added $(15 \mathrm{~mL})$ and shaken vigorously, forming a solid precipitate that was filtered, washed with $\mathrm{Et}_{2} \mathrm{O}(2 \times 15 \mathrm{~mL})$ and dried under high vacuum. If the pyridinium salt failed to precipitate, it was subjected to flash column chromatography, eluting with $\mathrm{DCM} /$ Acetone mixtures.

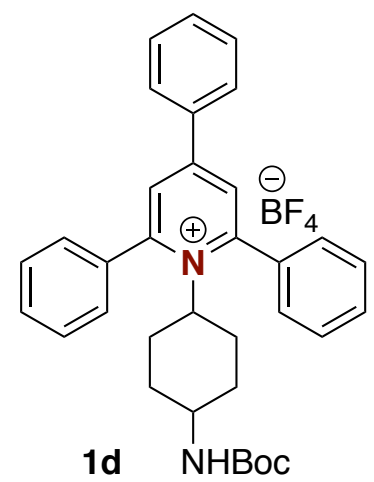

1-(4-((tert-butoxycarbonyl)amino)cyclohexyl)-2,4,6-triphenylpyridin-1-ium tetra-

fluoroborate (1d): Following the general procedure, but using tert-butyl (4aminocyclohexyl)carbamate (trans/cis $=1.5: 1$ ratio) $(1.28 \mathrm{~g}, 6 \mathrm{mmol})$, afforded $\mathbf{1 f}(1.78$ g, $60 \%$ yield) as a white solid. Mp $192-193{ }^{\circ} \mathrm{C} .{ }^{1} \mathbf{H}$ NMR $\left(400 \mathrm{MHz}, \mathrm{CDCl}_{3}\right): \delta 7.76$ 
- $7.64(\mathrm{~m}, 8 \mathrm{H}), 7.59-7.46(\mathrm{~m}, 7 \mathrm{H}), 7.39(\mathrm{td}, J=8.2,7.3,2.4 \mathrm{~Hz}, 2 \mathrm{H}), 4.56(\mathrm{t}, J=12.2$ $\mathrm{Hz}, 1 \mathrm{H}), 4.28-4.05$ (m, 1H), 2.88 (brs, 1H), 2.14 (d, $J=12.2 \mathrm{~Hz}, 2 \mathrm{H}), 1.80$ (d, $J=$ $12.8 \mathrm{~Hz}, 2 \mathrm{H}), 1.64-1.51(\mathrm{~m}, 2 \mathrm{H}), 1.38-1.27$ (s, 9H), 0.60 (q, $J=12.5 \mathrm{~Hz}, 2 \mathrm{H}) \mathrm{ppm}$. ${ }^{13}$ C NMR (101 MHz, $\left.\mathrm{CDCl}_{3}\right): \delta 157.1,155.2,155.0,134.1,134.0,132.0,131.1,129.6$, 129.4, 129.0, 128.4, 128.2 , 79.5, 70.6, 48.0, 33.0, 31.7, 28.4 ppm. ${ }^{19} \mathbf{F}$ NMR $(376 \mathrm{MHz}$, $\left.\mathrm{CDCl}_{3}\right) \delta-152.6(\mathrm{~s}),-152.7$ (s). ${ }^{11} \mathbf{B}$ NMR $\left(128 \mathrm{MHz}, \mathrm{CDCl}_{3}\right) \delta$-119.2. IR (neat, $\left.\mathrm{cm}^{-1}\right)$ : $3368,3244,2944,1682,1529,1181,1030,1026,853$. HRMS calcd. for $\left(\mathrm{C}_{34} \mathrm{H}_{37} \mathrm{~N}_{2} \mathrm{O}_{2}\right)$ $\left[\mathrm{M}-\mathrm{BF}_{4}\right]^{+}: 505.2850$ found 505.2866.

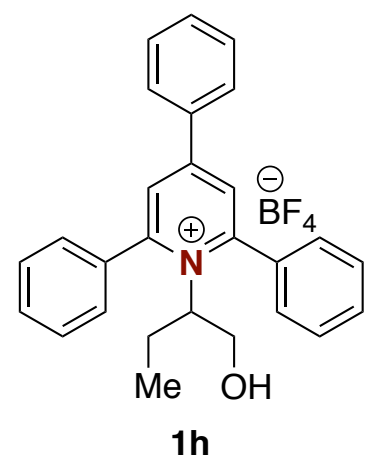

1-(1-hydroxybutan-2-yl)-2,4,6-triphenylpyridin-1-ium tetrafluoroborate (1h):

Following the general procedure, but using 2-aminobutan-1-ol $(0.54 \mathrm{~mL}, 6 \mathrm{mmol})$, afforded $\mathbf{1 h}$ (1.5 g, 65\% yield) as a white solid. Mp $97-98^{\circ} \mathrm{C} .{ }^{1} \mathbf{H}$ NMR (400 MHz, $\left.\mathrm{CDCl}_{3}\right): \delta 8.11-7.67(\mathrm{~m}, 6 \mathrm{H}), 7.66-7.29(\mathrm{~m}, 11 \mathrm{H}), 5.17-5.00(\mathrm{~m}, 1 \mathrm{H}), 3.81-3.64$ (m, 2H), $3.62-3.51(\mathrm{~m}, 1 \mathrm{H}), 1.72(\mathrm{dt}, J=14.6,7.3 \mathrm{~Hz}, 1 \mathrm{H}), 1.64(\mathrm{~s}, 1 \mathrm{H}), 1.46-1.38$ $(\mathrm{m}, 1 \mathrm{H}), 0.68(\mathrm{t}, J=7.4 \mathrm{~Hz}, 3 \mathrm{H}) \mathrm{ppm} .{ }^{13} \mathbf{C} \mathbf{N M R}\left(101 \mathrm{MHz}, \mathrm{CDCl}_{3}\right): \delta 155.3,133.7$, 132.5, 129.9, 128.4, 74.0, 63.1, 25.7, 11.2 ppm. ${ }^{19}$ F NMR (376 MHz, $\left.\mathrm{CDCl}_{3}\right) \delta-153.1$ (s), -153.2 (s) ppm. ${ }^{11} \mathbf{B}$ NMR (128 MHz, $\left.\mathrm{CDCl}_{3}\right) \delta-1.2 \mathrm{ppm}$. IR (neat, $\mathrm{cm}^{-1}$ ): 3524 , 3063, 2971, 2881, 1617, 1599, 1561, 1410, 1053, 1049, 890. HRMS calcd. for $\left(\mathrm{C}_{27} \mathrm{H}_{26} \mathrm{NO}\right)\left[\mathrm{M}-\mathrm{BF}_{4}\right]^{+}: 380.2009$ found 380.2000.

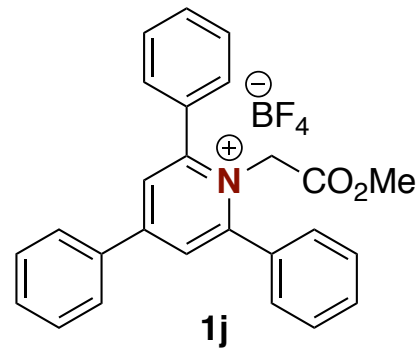

1-(2-methoxy-2-oxoethyl)-2,4,6-triphenylpyridin-1-ium tetrafluoroborate (1j): Following the general procedure, but using glycine methyl ester hydrochloride $(753 \mathrm{mg}$, $6.0 \mathrm{mmol})$ and $\mathrm{Et}_{3} \mathrm{~N}(0.83 \mathrm{~mL}, 6 \mathrm{mmol})$, afforded $\mathbf{1 j}$ (1.68 g, 72\% yield) as a white 
solid. Mp 183 - $184{ }^{\circ} \mathrm{C} .{ }^{1} \mathbf{H}$ NMR (400 MHz, $\left.\mathrm{CDCl}_{3}\right)$ : $\delta 7.96$ (s, 2H), $7.84-7.79(\mathrm{~m}$, 2H), 7.71 (brs, 3H), $7.63-7.50(\mathrm{~m}, 10 \mathrm{H}), 5.11$ (s, 2H), 3.54 (s, 3H) ppm. ${ }^{13} \mathbf{C}$ NMR (101 MHz, $\left.\mathrm{CDCl}_{3}\right): \delta 167.5,157.4,157.1,134.0,132.6,132.2,131.5,129.9,129.0$, 128.4, 126.3, 56.4, 53.4 ppm. ${ }^{19} \mathbf{F}$ NMR (376 MHz, $\left.\mathrm{CDCl}_{3}\right) \delta-153.0$ (s), -153.1(s) ppm. ${ }^{11} \mathrm{~B}$ NMR (128 MHz, $\left.\mathrm{CDCl}_{3}\right) \delta-119.1$ ppm. IR (neat, $\mathrm{cm}^{-1}$ ): 3041, 3010, 1752, 1619 , 1594, 1561, 1414, 1369, 1220, 1184, 1039, 997, 904. HRMS calcd. for $\left(\mathrm{C}_{26} \mathrm{H}_{22} \mathrm{NO}_{2}\right)$ $\left[\mathrm{M}-\mathrm{BF}_{4}\right]^{+}: 380.1645$ found 380.1642 .

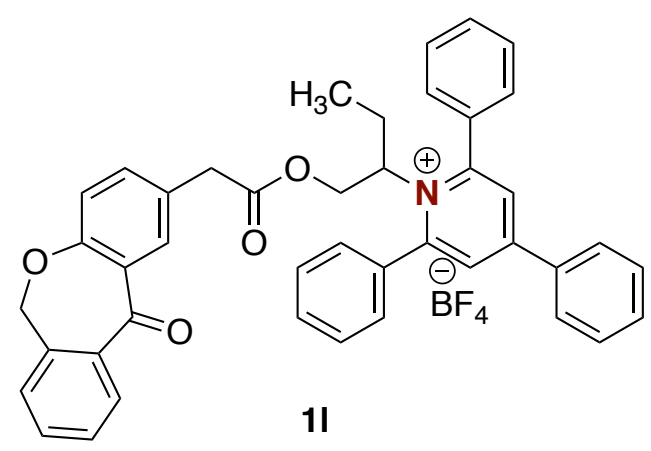

\section{1-(1-(2-(11-oxo-6,11-dihydrodibenzo[b,e]oxepin-2-yl)acetoxy)butan-2-yl)-2,4,6-}

triphenylpyridin-1-ium tetrafluoroborate (11): To a solution of 6,11-Dihydro-11oxodibenz[b,e]oxepin-2-acetic acid (1 mmol, $268 \mathrm{mg})$ and 1-(1-hydroxybutan-2-yl)2,4,6-triphenylpyridin-1-ium tetrafluoroborate (1h, $1 \mathrm{mmol}, 437 \mathrm{mg})$ in dry DCM (10 $\mathrm{mL})$ was added DCC (1 mmol, $206 \mathrm{mg})$ and DMAP $(0.05 \mathrm{mmol}, 6 \mathrm{mg})$. The reaction mixture was stirring at $\mathrm{rt}$ for $24 \mathrm{~h}$, then remove the solid by filter. The residue was purified by flash column chromatography $(\mathrm{DCM} /$ acetone $=10: 1)$ to provide $\mathbf{1 l}(516 \mathrm{mg}$, $72 \%)$ as a white solid. Mp $115-116^{\circ} \mathrm{C} .{ }^{1} \mathbf{H}$ NMR (400 MHz, $\left.\mathrm{CDCl}_{3}\right): \delta 8.00(\mathrm{~d}, J=$ $2.3 \mathrm{~Hz}, 1 \mathrm{H}), 7.94-7.65(\mathrm{~m}, 7 \mathrm{H}), 7.64-7.35$ (m, 12H), $7.34-7.25$ (m, 3H), 6.92 (d, $J$ $=8.4 \mathrm{~Hz}, 1 \mathrm{H}), 4.98(\mathrm{~d}, J=1.6 \mathrm{~Hz}, 2 \mathrm{H}), 4.95-4.86(\mathrm{~m}, 1 \mathrm{H}), 4.03-3.92(\mathrm{~m}, 2 \mathrm{H}), 3.59$ $(\mathrm{s}, 2 \mathrm{H}), 2.38-2.30(\mathrm{~m}, 1 \mathrm{H}), 1.60-1.48(\mathrm{~m}, 1 \mathrm{H}), 0.64(\mathrm{t}, J=7.3 \mathrm{~Hz}, 3 \mathrm{H}) \mathrm{ppm} .{ }^{13} \mathrm{C}$ NMR (101 MHz, $\left.\mathrm{CDCl}_{3}\right): \delta 190.5,170.1,160.7,155.7,140.1,136.2,135.6,133.6$, 133.2, 132.4, 132.3, 131.3, 129.7, 129.5, 128.6, 128.1, 127.0, 125.3, 121.5, 73.7, 69.9, 64.1, 40.2, 26.9, $11.5 \mathrm{ppm}$ (2 carbons missing due to signal broadening). ${ }^{19} \mathbf{F}$ NMR (376 $\mathrm{MHz}, \mathrm{CDCl}_{3}$ ) $\delta-153.08$ (s), -153.09 (s) ppm. ${ }^{11} \mathrm{~B} \mathrm{NMR}\left(128 \mathrm{MHz}, \mathrm{CDCl}_{3}\right) \delta-1.4 \mathrm{ppm}$. IR (neat, $\mathrm{cm}^{-1}$ ): 3061, 2940, 2928, 2852, 1738, 1644, 1617, 1598, 1562, 1490, 1412, 1300, 1242, 1139, 1050. HRMS calcd. for $\left(\mathrm{C}_{43} \mathrm{H}_{36} \mathrm{NO}_{4}\right)\left[\mathrm{M}-\mathrm{BF}_{4}\right]^{+}: 630.2639$ found 630.2630 . 


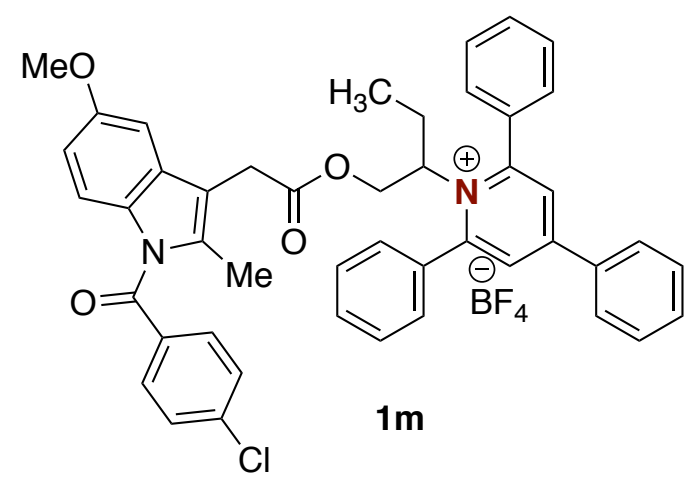

1-(1-(2-(1-(4-chlorobenzoyl)-5-methoxy-2-methyl-1H-indol-3-yl)acetoxy)butan-2yl)-2,4,6-triphenylpyridin-1-ium tetrafluoroborate $(1 \mathrm{~m})$ : To a solution of 1-(4Chlorobenzoyl)-5-methoxy-2-methyl-3-indoleacetic Acid(2 mmol, $717.6 \mathrm{mg})$ and 1(1-hydroxybutan-2-yl)-2,4,6-triphenylpyridin-1-ium tetrafluoroborate (1h, 2mmol, 934 $\mathrm{mg})$ in dry DCM $(20 \mathrm{~mL})$ was added DCC (2 mmol, $412 \mathrm{mg})$ and DMAP (0.1 mmol, $12 \mathrm{mg}$ ). The reaction mixture was stirring at $\mathrm{rt}$ for $24 \mathrm{~h}$, then remove the solid by filter. The residue was purified by flash column chromatography $(\mathrm{DCM} /$ acetone $=10: 1)$ to provide 1m (1.2 mg, 74\%) as a yellow solid. Mp $149-150{ }^{\circ} \mathrm{C} .{ }^{1} \mathbf{H}$ NMR (400 MHz, $\left.\mathrm{CDCl}_{3}\right): \delta 7.89-7.30(\mathrm{~m}, 20 \mathrm{H}), 7.03(\mathrm{~s}, 1 \mathrm{H}), 6.83(\mathrm{~d}, J=2.5 \mathrm{~Hz}, 1 \mathrm{H}), 6.69(\mathrm{~d}, J=9.0$ $\mathrm{Hz}, 1 \mathrm{H}), 6.52(\mathrm{dd}, J=9.0,2.5 \mathrm{~Hz}, 1 \mathrm{H}), 4.91-4.84(\mathrm{~m}, J=9.7,5.2 \mathrm{~Hz}, 1 \mathrm{H}), 4.03$ (dd, $J=12.6,5.5 \mathrm{~Hz}, 1 \mathrm{H}), 3.86-3.79(\mathrm{~m}, 1 \mathrm{H}), 3.71-3.63(\mathrm{~s}, 5 \mathrm{H}), 2.29-2.23(\mathrm{~m}, 4 \mathrm{H})$, $1.61-1.48(\mathrm{~m}, 1 \mathrm{H}), 0.60(\mathrm{t}, J=7.4 \mathrm{~Hz}, 3 \mathrm{H}) \mathrm{ppm} .{ }^{13} \mathrm{C}$ NMR $\left(101 \mathrm{MHz}, \mathrm{CDCl}_{3}\right): \delta$ $169.7,168.2$, 156.1 155.5, 139.9, 136.1, 133.6, 133.3, 132.4, 131.3, 130.7, 130.4, 129.7, $129.3,128.8,128.7,128.6,114.9,111.8,111.5,101.8,69.6,64.0,55.9,30.2,26.9,13.2$, 11.4 ppm. ${ }^{19}$ F NMR $\left(376 \mathrm{MHz}, \mathrm{CDCl}_{3}\right) \delta-152.8(\mathrm{~s}),-152.9(\mathrm{~s}) \mathrm{ppm} .{ }^{11} \mathrm{~B}$ NMR (128 $\mathrm{MHz}, \mathrm{CDCl}_{3}$ ) $\delta-1.38$ ppm. IR (neat, $\mathrm{cm}^{-1}$ ): 3064, 2931, 2852, 1739, 1679, 1617, 1598, 1561, 1477, 1457, 1355, 1315, 1256, 1223, 1143, 1051, 1030. HRMS calcd. for $\left(\mathrm{C}_{46} \mathrm{H}_{40} \mathrm{ClN}_{2} \mathrm{O}_{4}\right)\left[\mathrm{M}-\mathrm{BF}_{4}\right]^{+}: 719.2671$ found 719.2690 .

\section{Procedure for the Preparation of Olefins}

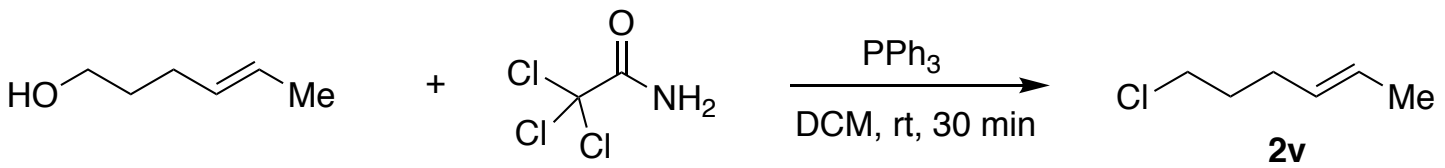

6-chloro-2-hexene (2v): To a stirred solution of the (E)-hex-4-en-1-ol (0.59 mL, $5.0 \mathrm{mmol})$ and $\mathrm{PPh}_{3}(2.62 \mathrm{~g}, 10.0 \mathrm{mmol})$ in dry DCM $(10 \mathrm{~mL})$ was added 2,2,2-trichloroacetamide (1.62 $\mathrm{g}, 10.0 \mathrm{mmol})$ under Ar. The reaction mixture was stirring at $\mathrm{rt}$ for $30 \mathrm{~min}$, then quenched with cold water. The aqueous phase was extracted with DCM (3 x $10 \mathrm{~mL})$, and 
the combined organic phases were dried over $\mathrm{MgSO}_{4}$ then concentrated. The residue was purified by flash column chromatography (Hexane/EtOAc $=100: 1)$ to provide $2 \mathbf{v}(390 \mathrm{mg}$, $66 \%$ ) as a colorless oil. ${ }^{1} \mathbf{H}$ NMR $\left(400 \mathrm{MHz}, \mathrm{CDCl}_{3}\right): \delta 5.56-5.43(\mathrm{~m}, 1 \mathrm{H}), 5.43-5.31$ $(\mathrm{m}, 1 \mathrm{H}), 3.53(\mathrm{t}, J=6.7 \mathrm{~Hz}, 2 \mathrm{H}), 2.16-2.10(\mathrm{~m}, 2 \mathrm{H}), 1.85-1.78(\mathrm{~m}, 2 \mathrm{H}), 1.65(\mathrm{dq}, J=$ 6.3, 1.3 Hz, 3H) ppm. ${ }^{13} \mathbf{C}$ NMR (101 MHz, $\left.\mathrm{CDCl}_{3}\right): \delta 129.5,126.5,44.6,32.5,29.8,18.0$ ppm. IR (neat, $\mathrm{cm}^{-1}$ ): 2959, 2936, 2919, 2855, 1441, 1561, 1414, 1283, 965, 734, 653. GCMS: $\left(\mathrm{C}_{6} \mathrm{H}_{11} \mathrm{Cl}\right)[\mathrm{M}]+$ : found $\mathrm{t}=4.663 \mathrm{~min}, \mathrm{~m} / \mathrm{z} 118.1$.<smiles>CC=CC(CCC=C(C)C)CCC(C)C(C)(C)OCC</smiles>

2,6-dimethylnona-2,7-diene (2w): To a stirred solution of the ethyltriphenylphosphonium iodide (4.6 g, $11.0 \mathrm{mmol})$ and $t$-BuOK $(1.23 \mathrm{~g}, 11.0 \mathrm{mmol})$ in $\mathrm{Et}_{2} \mathrm{O}(30 \mathrm{~mL})$ was added 2,6-dimethylhept-5-enal $(1.59 \mathrm{~mL}, 10.0 \mathrm{mmol})$ by syringe. The reaction mixture was refluxed for $12 \mathrm{~h}$. The crude reaction mixture was purified by flash column chromatography $($ Hexane/EtOAc $=300: 1)$ to provide $\mathbf{2 w}(1.08 \mathrm{~g}, 71 \%)$ as a colorless oil. ${ }^{1} \mathbf{H}$ NMR $\left(400 \mathrm{MHz}, \mathrm{CDCl}_{3}\right): \delta 5.43-5.35(\mathrm{~m}, 1 \mathrm{H}), 5.20-5.06(\mathrm{~m}$, $2 \mathrm{H}), 2.53-2.41(\mathrm{~m}, 1 \mathrm{H}), 2.00-1.88(\mathrm{~m}, 2 \mathrm{H}), 1.69(\mathrm{~d}, J=1.4 \mathrm{~Hz}, 3 \mathrm{H}), 1.60(\mathrm{dd}, J=$ 6.8, $1.8 \mathrm{~Hz}, 6 \mathrm{H}), 1.38-1.17(\mathrm{~m}, 2 \mathrm{H}), 0.94(\mathrm{~d}, J=6.7 \mathrm{~Hz}, 3 \mathrm{H}) \mathrm{ppm} .{ }^{13} \mathbf{C}$ NMR (101 $\left.\mathrm{MHz}_{2} \mathrm{CDCl}_{3}\right): \delta 137.3,131.3,125.0,122.5,37.8,31.1,26.2,25.9,21.2,17.8,13.1$. IR (neat, $\left.\mathrm{cm}^{-1}\right)$ : 2963, 2916, 2855, 1452, 1375, 967, 949, 720. HRMS calcd. for $\left(\mathrm{C}_{11} \mathrm{H}_{19}\right)$ $[\mathrm{M}-\mathrm{H}]^{+}: 151.1481$ found 151.1477.<smiles>C=CCCCCBr</smiles><smiles>C=CCCCCO[C@H](Cc1ccc(Cl)cc1)n1cncn1</smiles>

\section{1-((2R,3R)-1-(4-chlorophenyl)-3-(hex-5-en-1-yloxy)-4,4-dimethylpentan-2-yl)-}

1H-1,2,4-triazole (2ad): An oven-dried $50 \mathrm{~mL}$ flask containing a stirring bar was charged with Paclobutrazol (2.93 g, $10 \mathrm{mmol})$ and dry DMF (10 mL), cooling down to $0{ }^{\circ} \mathrm{C}$, the $60 \% \mathrm{NaH}$ ( $720 \mathrm{mg}, 30 \mathrm{mmol}$ ) was added slowly. The reaction mixture was stirring at $\mathrm{rt}$ for $15 \mathrm{~min}$ until $\mathrm{H}_{2}$ bubbling ceased. 6-bromo-1-hexene (1.55 mL, 11 mmol) was added dropwise and the reaction stirred overnight. The reaction mixture 
was quenched by saturated $\mathrm{NH}_{4} \mathrm{Cl}$ under $0{ }^{\circ} \mathrm{C}$, extracted with $\mathrm{DCM}$, dried over $\mathrm{MgSO}_{4}$ and the volatiles were removed under vacuum. Flash column chromatography (hexanes/ethyl acetate 5:1) of the crude mixture afforded the title compound $2.36 \mathrm{~g}$ (63\%) as white solid. Mp 54.5 - 55.6 ${ }^{\circ} \mathrm{C} .{ }^{1} \mathbf{H}$ NMR (400 MHz, $\left.\mathrm{CDCl}_{3}\right): \delta 8.38(\mathrm{~s}, 1 \mathrm{H})$, $7.75(\mathrm{~d}, J=2.2 \mathrm{~Hz}, 1 \mathrm{H}), 7.18(\mathrm{dd}, J=8.5,2.1 \mathrm{~Hz}, 2 \mathrm{H}), 6.92(\mathrm{dd}, J=8.4,1.8 \mathrm{~Hz}, 2 \mathrm{H})$, $5.87-5.77(\mathrm{~m}, 1 \mathrm{H}), 5.08-4.95(\mathrm{~m}, 2 \mathrm{H}), 4.82-4.77(\mathrm{~m}, 1 \mathrm{H}), 3.69(\mathrm{td}, J=6.4,1.5 \mathrm{~Hz}$, $2 \mathrm{H}), 3.27-3.05(\mathrm{~m}, 3 \mathrm{H}), 2.14-2.08(\mathrm{~m}, 2 \mathrm{H}), 1.75-1.62(\mathrm{~m}, 2 \mathrm{H}), 1.60-1.48(\mathrm{~m}$, $2 \mathrm{H}), 0.74(\mathrm{~d}, J=1.5 \mathrm{~Hz}, 9 \mathrm{H}) \mathrm{ppm} .{ }^{13} \mathbf{C} \mathbf{N M R}\left(101 \mathrm{MHz}, \mathrm{CDCl}_{3}\right): \delta 150.0,143.7,138.5$, 135.4, 133.0, 130.0, 129.0, 115.0, 87.3, 75.5, 63.6, 40.9, 36.4, 33.7, 30.1, 26.6, 25.8 ppm. IR (neat, $\mathrm{cm}^{-1}$ ): 2973, 2938, 2897, 2874, 1504, 1491, 1370, 1267, 1139, 1088, 1014, 906, 754. HRMS calcd. for $\left(\mathrm{C}_{21} \mathrm{H}_{31} \mathrm{ClN}_{3} \mathrm{O}\right)[\mathrm{M}+\mathrm{H}]^{+}: 376.2150$ found 376.2149 .<smiles>CC1(C)OC2C3OC(C)(C)O[C@H]3C(CO)O[C@H]2O1</smiles><smiles>C=CCCCCBr</smiles><smiles>C=CCCCOCC1O[C@@H]2OC(C)(C)O[C@H]2C2OC(C)(C)OC12</smiles>

(3aR,5R,5aS,8aS,8bR)-5-((hex-5-en-1-yloxy)methyl)-2,2,7,7

tetramethyltetrahydro-5H-bis([1,3]dioxolo) $\left[4,5-b: 4^{\prime}, 5^{\prime}-d\right]$ pyran (2ae): An ovendried $50 \mathrm{~mL}$ flask containing a stirring bar was charged with 1,2:3,4-Di-Oisopropylidene- $\alpha$-D-galactopyranose $(2.6 \mathrm{~g}, 10 \mathrm{mmol})$ and dry DMF $(10 \mathrm{~mL})$, cooling down to $0{ }^{\circ} \mathrm{C}$, the $60 \% \mathrm{NaH}$ ( $720 \mathrm{mg}, 30 \mathrm{mmol}$ ) was added slowly. The reaction mixture was stirring at $\mathrm{rt}$ for $15 \mathrm{~min}$ until $\mathrm{H}_{2}$ bubbling ceased. 6-bromo-1-hexene (1.55 mL, 11 mool) was added dropwise and the reaction stirred overnight. The reaction mixture was quenched by saturated $\mathrm{NH}_{4} \mathrm{Cl}$ under $0{ }^{\circ} \mathrm{C}$, extracted with $\mathrm{DCM}$, dried over $\mathrm{MgSO}_{4}$ and the volatiles were removed under vacuum. Flash column chromatography (hexanes/ethyl acetate 5:1) of the crude mixture afforded the title compound $2.4 \mathrm{~g}$ (70\%) as colorless. ${ }^{1} \mathbf{H}$ NMR $\left(400 \mathrm{MHz}, \mathrm{CDCl}_{3}\right): \delta 4.82-5.72(\mathrm{~m}, 1 \mathrm{H}), 5.50(\mathrm{~d}, J=$ $5.0 \mathrm{~Hz}, 1 \mathrm{H}), 5.02-4.87(\mathrm{~m}, 2 \mathrm{H}), 4.57(\mathrm{dd}, J=7.9,2.4 \mathrm{~Hz}, 1 \mathrm{H}), 4.33-4.20(\mathrm{~m}, 2 \mathrm{H})$, $3.93(\mathrm{td}, J=6.3,1.9 \mathrm{~Hz}, 1 \mathrm{H}), 3.63-3.40(\mathrm{~m}, 4 \mathrm{H}), 2.07-2.01(\mathrm{~m}, 2 \mathrm{H}), 1.61-1.54(\mathrm{~m}$, 2H), $1.51(\mathrm{~s}, 3 \mathrm{H}), 1.46-1.38(\mathrm{~m}, 5 \mathrm{H}), 1.34-1.27(\mathrm{~m}, 6 \mathrm{H}) \mathrm{ppm} .{ }^{13} \mathbf{C} \mathbf{N M R}(101 \mathrm{MHz}$, $\left.\mathrm{CDCl}_{3}\right): \delta 139.0,114.6,109.3,108.7,96.5,71.5,71.4,70.81,70.80,69.5,66.9,33.7$, 29.2, 26.2, 26.14, 25.5, 25.1, 24.6 ppm. IR (neat, $\left.\mathrm{cm}^{-1}\right)$ : 2982, 2934, 2865, 1640, 1380, 1254, 1210, 1111, 1068, 999, 916. HRMS calcd. for $\left(\mathrm{C}_{18} \mathrm{H}_{30} \mathrm{NaO}_{6}\right)[\mathrm{M}+\mathrm{Na}]^{+}: 365.1935$ found 365.1937 . 


\section{Ni-Catalyzed Deaminative Alkylation with Terminal Olefins (Table 2)}

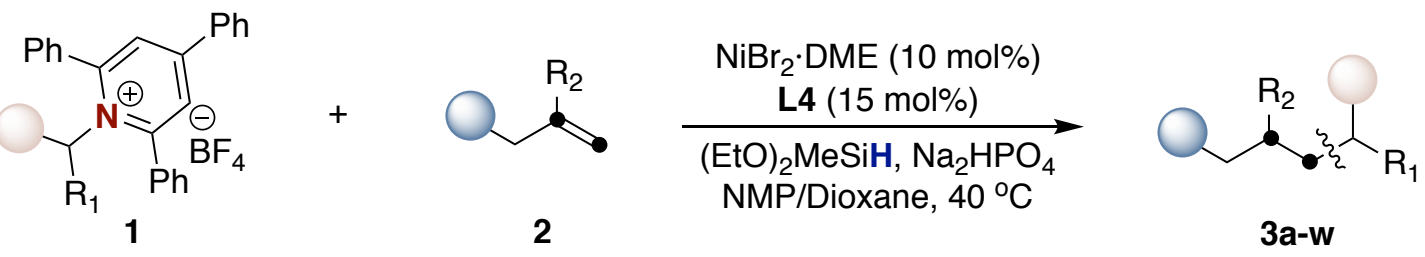

General procedure: An oven-dried $8 \mathrm{~mL}$ screw-cap test tube containing a stirring bar was charged with $\mathrm{NiBr}_{2} \cdot \mathrm{DME}$ (6.2 mg, $10 \mathrm{~mol} \%$ ), 4,4'-di-tert-butyl-2,2'-dipyridyl (L4, $8.0 \mathrm{mg}$, $15 \mathrm{~mol} \%), \mathrm{Na}_{2} \mathrm{HPO}_{4}(56.8 \mathrm{mg}, 0.40 \mathrm{mmol})$ and pyridinium salt (1, $\left.0.20 \mathrm{mmol}\right)$. Subsequently, the tube was sealed with a Teflon-lined screw cap, then evacuated and backfilled with $\operatorname{Ar}$ (3 times). Afterwards, $\alpha$-olefin (2, 0.6 mmol), (EtO) ${ }_{2} \mathrm{MeSiH}$ (DEMS, 96 ul, $0.60 \mathrm{mmol})$, NMP and 1.4-dioxane $(4: 1,1.0 \mathrm{~mL})$ were added via syringe. Then, the tube was stirred at $40{ }^{\circ} \mathrm{C}$ for $24 \mathrm{~h}$. After the reaction was completed, the mixture was diluted with EtOAc, filtered through silica gel and concentrated under vacuum. The corresponding product was purified by column chromatography on silica gel. Note: if the corresponding product possesses a similar $R_{f}$ to that of 2,4,6-triphenylpyridine, then toluene should be used as eluent followed by hexane/EtOAc mixtures.

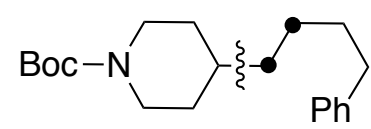

3a

tert-butyl 4-(4-phenylbutyl)piperidine-1-carboxylate (3a): Following the general procedure, but using 4-phenyl-1-butene (90 $\mu \mathrm{L}, 0.60 \mathrm{mmol}$ ), afforded 3a (51 mg, 80\% yield) as a colorless oil. In an independent experiment, $46 \mathrm{mg}$ (73\% yield) were obtained, giving an average of $76 \%$ yield. ${ }^{1} \mathbf{H}$ NMR (400 $\mathrm{MHz}, \mathrm{CDCl}_{3}$ ): $\delta 7.31-7.25$ (m, 2H), $7.21-7.14(\mathrm{~m}, 3 \mathrm{H}), 4.08(\mathrm{br}, 2 \mathrm{H}), 2.66(\mathrm{t}, J=12.8 \mathrm{~Hz}, 2 \mathrm{H}), 2.62(\mathrm{t}, J=3.6$ $\mathrm{Hz}, 2 \mathrm{H}), 1.72-1.56(\mathrm{~m}, 4 \mathrm{H}), 1.47$ (s, 9H), $1.40-1.30(\mathrm{~m}, 3 \mathrm{H}), 1.30-1.23(\mathrm{~m}, 2 \mathrm{H})$, $1.12-1.02(\mathrm{~m}, 2 \mathrm{H}) \mathrm{ppm} .{ }^{13} \mathbf{C} \mathbf{N M R}\left(101 \mathrm{MHz}, \mathrm{CDCl}_{3}\right): \delta 154.9,142.7,128.4,128.3$, 125.7, 79.1, 44.1, 36.4, 36.0, 35.9, 32.3, 31.7, 28.5, 26.3 ppm. IR (neat, $\mathrm{cm}^{-1}$ ): 2975, 2927, 2853, 1689, 1419, 1364, 1277, 1239, 1154, 866. HRMS calcd. for $\left(\mathrm{C}_{20} \mathrm{H}_{31} \mathrm{NNaO}_{2}\right)[\mathrm{M}+\mathrm{Na}]^{+}: 340.2247$ found 340.2244 . 


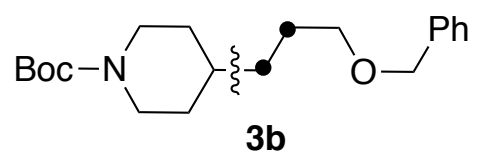

tert-butyl 4-(3-(benzyloxy)propyl)piperidine-1-carboxylate (3b): Following the general procedure, but using ((allyloxy)methyl)benzene ( $88.8 \mathrm{mg}, 0.60 \mathrm{mmol})$, afforded $3 \mathbf{b}$ (44 mg, 66\% yield) as a colorless oil. In an independent experiment, $42 \mathrm{mg}$ (63\% yield) were obtained, giving an average of 64\% yield. ${ }^{1} \mathbf{H}$ NMR (400 MHz, $\left.\mathrm{CDCl}_{3}\right) \delta 7.38-7.27(\mathrm{~m}, 5 \mathrm{H}), 4.50(\mathrm{~s}, 2 \mathrm{H}), 4.06(\mathrm{~d}, J=13.1 \mathrm{~Hz}, 2 \mathrm{H}), 3.46(\mathrm{t}, J=6.6$ $\mathrm{Hz}, 2 \mathrm{H}), 2.65$ (t, $J=11.9 \mathrm{~Hz}, 2 \mathrm{H}), 1.72-1.59$ (m, 4H), 1.45 (s, 9H), $1.39-1.26$ (m, $3 \mathrm{H}), 1.11-1.05(\mathrm{~m}, 2 \mathrm{H}) \mathrm{ppm} .{ }^{13} \mathbf{C}$ NMR $\left(101 \mathrm{MHz}, \mathrm{CDCl}_{3}\right): \delta 155.0,138.7,128.5$, 127.7, 127.6, 79.3, 73.0, 70.6, 44.2, 36.0, 33.1, 32.3, 28.6, 27.0 ppm. IR (neat, $\mathrm{cm}^{-1}$ ): 2974, 2929, 2851, 1688, 1420, 1364, 1277, 1242, 1167, 1145, 1097, 938, 864. HRMS calcd. for $\left(\mathrm{C}_{20} \mathrm{H}_{31} \mathrm{NNaO}_{3}\right)[\mathrm{M}+\mathrm{Na}]^{+}: 356.2196$ found 356.2199.

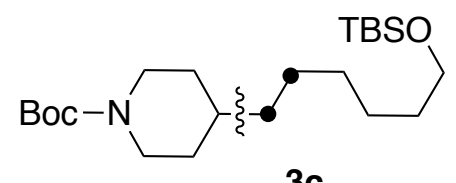

$3 c$

tert-butyl 4-(6-((tert-butyldimethylsilyl)oxy)hexyl)piperidine-1-carboxylate (3c):

Method A: Following the general procedure, but using tert-butyl(hex-5-en-1yloxy)dimethylsilane (128.4 $\mathrm{mg}, 0.60 \mathrm{mmol})$, afforded $3 \mathbf{c}(58 \mathrm{mg}, 73 \%$ yield) as a colorless oil. In an independent experiment, $60 \mathrm{mg}$ (75\% yield) were obtained, giving an average of $74 \%$ yield.

Method B: Following the general procedure, but using $\mathrm{NiI}_{2}(6.2 \mathrm{mg}, 10 \mathrm{~mol} \%)$, 2-(6methylpyridin-2-yl)-4,5-dihydrooxazole (L6, $6.4 \mathrm{mg}, 20 \mathrm{~mol} \%$ ), $\mathrm{Na}_{2} \mathrm{HPO}_{4}$ (113.6 mg, $0.80 \mathrm{mmol}),(\mathrm{EtO})_{3} \mathrm{SiH}(74 \mu \mathrm{L}, 0.40 \mathrm{mmol})$ in $\mathrm{DMSO} /$ dioxane and $(E)$-tert-butyl(hex4-en-1-yloxy)dimethylsilane (2u, $128.4 \mathrm{mg}, 0.60 \mathrm{mmol}$ ), afforded $\mathbf{3 c}$ (38 mg, 48\% yield) as a colorless oil. In an independent experiment, $41 \mathrm{mg}$ (52\% yield) were obtained, giving an average of 50\% yield, $48: 1$ ratio. ${ }^{1} \mathbf{H}$ NMR $\left(400 \mathrm{MHz}, \mathrm{CDCl}_{3}\right) \delta$ $4.05(\mathrm{~d}, J=13.1 \mathrm{~Hz}, 2 \mathrm{H}), 3.59$ (t, $J=6.6 \mathrm{~Hz}, 2 \mathrm{H}), 2.65(\mathrm{t}, J=12.3 \mathrm{~Hz}, 2 \mathrm{H}), 1.63$ (d, $J$ $=12.9 \mathrm{~Hz}, 2 \mathrm{H}), 1.49(\mathrm{t}, J=6.5 \mathrm{~Hz}, 2 \mathrm{H}), 1.44(\mathrm{~s}, 9 \mathrm{H}), 1.37-1.14(\mathrm{~m}, 9 \mathrm{H}), 1.11-0.96$ $(\mathrm{m}, 2 \mathrm{H}), 0.88(\mathrm{~s}, 9 \mathrm{H}), 0.04(\mathrm{~s}, 6 \mathrm{H}) \mathrm{ppm} .{ }^{13} \mathrm{C}$ NMR (101 MHz, $\left.\mathrm{CDCl}_{3}\right): \delta$ 155.1, 79.2, 63.4, 44.2, 36.6, 36.1, 33.0, 32.4, 29.7, 28.6, 26.7, 26.1, 25.9, 18.5, -5.1 ppm. IR (neat, $\mathrm{cm}^{-1}$ ): 2975, 2927, 2855, 1695, 1420, 1364, 1247, 1160, 1097, 834. HRMS calcd. for $\left(\mathrm{C}_{22} \mathrm{H}_{45} \mathrm{NNaO}_{3} \mathrm{Si}\right)[\mathrm{M}+\mathrm{Na}]^{+}: 422.3061$ found 422.3069. 


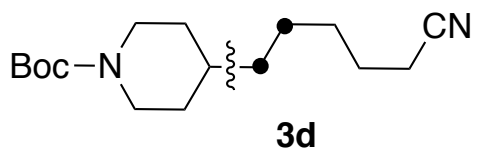

tert-butyl 4-(5-cyanopentyl)piperidine-1-carboxylate (3d): Following the general procedure, but using hex-5-enenitrile $(68.1 \mu \mathrm{L}, 0.60 \mathrm{mmol})$, afforded $3 \mathbf{d}(39 \mathrm{mg}, 70 \%$ yield) as a light yellow oil. In an independent experiment, $38 \mathrm{mg}$ (68\% yield) were obtained, giving an average of $69 \%$ yield. ${ }^{1} \mathbf{H}$ NMR $\left(400 \mathrm{MHz}, \mathrm{CDCl}_{3}\right) \delta 4.05(\mathrm{~d}, J=$ $13.4 \mathrm{~Hz}, 2 \mathrm{H}), 2.64$ (t, $J=12.8 \mathrm{~Hz}, 2 \mathrm{H}), 2.32$ (t, $J=7.1 \mathrm{~Hz}, 2 \mathrm{H}), 1.75-1.55$ (m, 4H), $1.43(\mathrm{~s}, 9 \mathrm{H}), 1.43-1.38(\mathrm{~m}, 2 \mathrm{H}), 1.38-1.28$ (m, 3H), $1.27-1.19(\mathrm{~m}, 2 \mathrm{H}), 1.10-0.99$ $(\mathrm{m}, 2 \mathrm{H}) \mathrm{ppm} .{ }^{13} \mathbf{C} \mathbf{N M R}\left(101 \mathrm{MHz}, \mathrm{CDCl}_{3}\right) \delta 155.0,119.8,79.3,44.1,36.3,36.0,32.2$, 28.9, 28.6, 25.9, 25.4, 17.2 ppm. IR (neat, $\mathrm{cm}^{-1}$ ): 2974, 2927, 2854, 1687, 1421, 1365, 1276, 1245, 1155, 1088, 865. HRMS calcd. for $\left(\mathrm{C}_{16} \mathrm{H}_{28} \mathrm{~N}_{2} \mathrm{NaO}_{2}\right)[\mathrm{M}+\mathrm{Na}]^{+}: 303.2043$ found 303.2044.

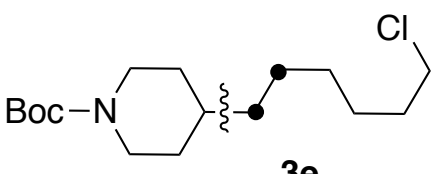

$3 e$

tert-butyl 4-(6-chlorohexyl)piperidine-1-carboxylate (3e): Method A: Following the general procedure, but using 6-chloro-1-hexene (79 $\mu \mathrm{L}, 0.60 \mathrm{mmol})$, afforded $3 \mathbf{e}$ (40 $\mathrm{mg}, 66 \%$ yield) as a colorless oil. In an independent experiment, $40 \mathrm{mg}$ (66\% yield) were obtained, giving an average of $66 \%$ yield.

Method B: Following the general procedure, but using $\mathrm{NiI}_{2}(6.2 \mathrm{mg}, 10 \mathrm{~mol} \%)$, 2-(6methylpyridin-2-yl)-4,5-dihydrooxazole (L6, $6.4 \mathrm{mg}, 20$ mol\%), $\mathrm{Na}_{2} \mathrm{HPO}_{4}$ (113.6 mg, $0.80 \mathrm{mmol}),(\mathrm{EtO})_{3} \mathrm{SiH}(74 \mu \mathrm{L}, 0.40 \mathrm{mmol})$ in DMSO/dioxane and (Z)-6-chloro-2hexene (2v, $70.8 \mathrm{mg}, 0.60 \mathrm{mmol})$, afforded $3 \mathbf{e}(30 \mathrm{mg}, 50 \%$ yield) as a colorless oil. In an independent experiment, $27 \mathrm{mg}$ (45\% yield) were obtained, giving an average of $47 \%$ yield, 28:1 ratio. ${ }^{1} \mathbf{H}$ NMR $\left(400 \mathrm{MHz}, \mathrm{CDCl}_{3}\right) \delta 4.05(\mathrm{~d}, J=13.2 \mathrm{~Hz}, 2 \mathrm{H}), 3.51(\mathrm{t}, J=$ $6.7 \mathrm{~Hz}, 2 \mathrm{H}), 2.65(\mathrm{t}, J=12.7 \mathrm{~Hz}, 2 \mathrm{H}), 1.79-1.72(\mathrm{~m}, 2 \mathrm{H}), 1.66-1.58(\mathrm{~m}, 2 \mathrm{H}), 1.44$ (s, 9H), $1.42-1.37(\mathrm{~m}, 2 \mathrm{H}), 1.35-1.25(\mathrm{~m}, 5 \mathrm{H}), 1.23-1.19(\mathrm{~m}, 2 \mathrm{H}), 1.10-0.99(\mathrm{~m}$, 2H) ppm. ${ }^{13} \mathbf{C}$ NMR $\left(101 \mathrm{MHz}, \mathrm{CDCl}_{3}\right) \delta 155.0,79.2,45.2,44.2,36.5,36.1,32.7,32.3$, 29.2, 28.6, 27.0, 26.6 ppm. IR (neat, $\mathrm{cm}^{-1}$ ): 2975, 2926, 2853, 1688, 1419, 1364, 1277 , 1242, 1153, 965, 868. HRMS calcd. for $\left(\mathrm{C}_{16} \mathrm{H}_{30} \mathrm{ClNNaO}_{2}\right)[\mathrm{M}+\mathrm{Na}]^{+}: 326.1857$ found 326.1859 . 


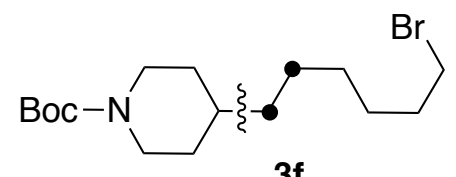

$3 f$

tert-butyl 4-(6-bromohexyl)piperidine-1-carboxylate (3f): Following the general procedure, but using 6-bromo-1-hexene ( $80 \mu \mathrm{L}, 0.60 \mathrm{mmol}$ ), afforded $\mathbf{3 f}$ (49 $\mathrm{mg}, 71 \%$ yield) as a colorless oil. In an independent experiment, $52 \mathrm{mg}$ (75\% yield) were obtained, giving an average of $73 \%$ yield. ${ }^{1} \mathbf{H}$ NMR (400 MHz, $\left.\mathrm{CDCl}_{3}\right) \delta 4.05(\mathrm{~d}, J=$ $13.3 \mathrm{~Hz}, 2 \mathrm{H}), 3.39$ (t, $J=6.8 \mathrm{~Hz}, 2 \mathrm{H}), 2.65(\mathrm{td}, J=12.7,2.7 \mathrm{~Hz}, 2 \mathrm{H}), 1.93-1.77(\mathrm{~m}$, $2 \mathrm{H}), 1.62(\mathrm{dt}, J=13.3,3.1 \mathrm{~Hz}, 2 \mathrm{H}), 1.44(\mathrm{~s}, 9 \mathrm{H}), 1.43-1.37(\mathrm{~m}, 2 \mathrm{H}), 1.36-1.27(\mathrm{~m}$, $5 \mathrm{H}), 1.25-1.16(\mathrm{~m}, 2 \mathrm{H}), 1.10-1.00(\mathrm{~m}, 2 \mathrm{H}) \mathrm{ppm} .{ }^{13} \mathbf{C ~ N M R}\left(101 \mathrm{MHz}, \mathrm{CDCl}_{3}\right) \delta$ 155.0, 79.3, 44.2, 36.5, 36.1, 34.1, 32.9, 32.3, 29.1, 28.6, 28.3, 26.5 ppm. IR (neat, cm 1): 2974, 2926, 2853, 1689, 1420, 1364, 1276, 1242, 1154, 964, 868. HRMS calcd. for $\left(\mathrm{C}_{16} \mathrm{H}_{30} \mathrm{BrNNaO}_{2}\right)[\mathrm{M}+\mathrm{Na}]^{+}: 370.1352$ found 370.1356 .

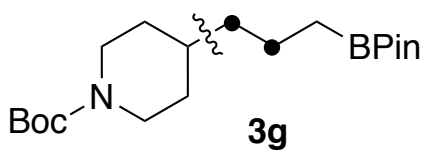

tert-butyl 4-(3-(4,4,5,5-tetramethyl-1,3,2-dioxaborolan-2-yl)propyl)piperidine-1carboxylate (3g): Following the general procedure, but using 2-allyl-4,4,5,5tetramethyl-1,3,2-dioxaborolane (112.6 $\mu \mathrm{L}, 0.60 \mathrm{mmol})$, afforded $3 \mathrm{~g}$ (40 $\mathrm{mg}, 56 \%$ yield) as a colorless oil. In an independent experiment, $41 \mathrm{mg}$ (58\% yield) were obtained, giving an average of $57 \%$ yield. ${ }^{1} \mathbf{H}$ NMR $\left(400 \mathrm{MHz}, \mathrm{CDCl}_{3}\right) \delta 4.03(\mathrm{~d}, J=13.2 \mathrm{~Hz}$, 2H), $2.64(\mathrm{td}, J=14,2.8 \mathrm{~Hz}, 2 \mathrm{H}), 1.72-1.56(\mathrm{~m}, 2 \mathrm{H}), 1.43(\mathrm{~s}, 9 \mathrm{H}), 1.42-1.29$ (m, $3 \mathrm{H}), 1.22(\mathrm{~s}, 12 \mathrm{H}), 1.19(\mathrm{t}, J=3.3 \mathrm{~Hz}, 2 \mathrm{H}), 1.09-0.98(\mathrm{~m}, 2 \mathrm{H}), 0.73(\mathrm{t}, J=7.8 \mathrm{~Hz}$, 2H) ppm. ${ }^{13} \mathbf{C}$ NMR $\left(101 \mathrm{MHz}, \mathrm{CDCl}_{3}\right) \delta 155.1,83.0,79.2,44.2,39.4,36.8,35.9,32.3$, 28.6, 24.9, 21.2 ppm. ${ }^{11} \mathbf{B}$ NMR (128 MHz, $\left.\mathrm{CDCl}_{3}\right) \delta 34.2 \mathrm{ppm}$. IR (neat, $\mathrm{cm}^{-1}$ ): 2976, 2927, 2859, 1693, 1420, 1365, 1319, 1276, 1246, 1144, 966, 847. HRMS calcd. for $\left(\mathrm{C}_{19} \mathrm{H}_{36} \mathrm{NNaBO}_{4}\right)[\mathrm{M}+\mathrm{Na}]^{+}: 375.2666$ found 375.2666. 


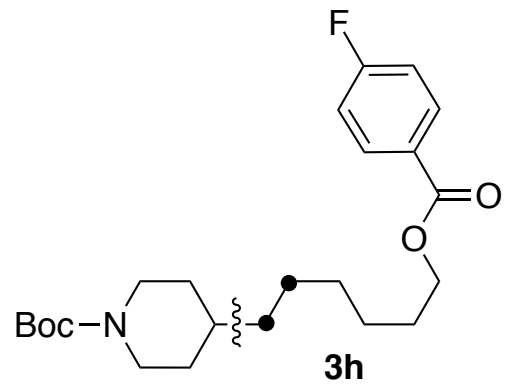

tert-butyl 4-(6-((4-fluorobenzoyl)oxy)hexyl)piperidine-1-carboxylate (3h): Following the general procedure, but using hex-5-en-1-yl 4-fluorobenzoate (133.2 mg, 0.60 mmol), afforded $\mathbf{3 h}$ (62 mg, 77\% yield) as a light yellow oil. In an independent experiment, $62 \mathrm{mg}$ (77\% yield) were obtained, giving an average of $77 \%$ yield. ${ }^{1} \mathbf{H}$ NMR (400 MHz, CDCl3) $\delta 8.02(\mathrm{dd}, \mathrm{J}=8.9,5.4 \mathrm{~Hz}, 2 \mathrm{H}), 7.07$ (t, J = 8.7 Hz, 2H), 4.27 $(\mathrm{t}, \mathrm{J}=6.7 \mathrm{~Hz}, 2 \mathrm{H}), 4.03(\mathrm{~d}, \mathrm{~J}=13.2 \mathrm{~Hz}, 2 \mathrm{H}), 2.73-2.56(\mathrm{~m}, 2 \mathrm{H}), 1.77-1.68(\mathrm{~m}, 2 \mathrm{H})$, $1.65-1.55(\mathrm{~m}, 2 \mathrm{H}), 1.42(\mathrm{~s}, 9 \mathrm{H}), 1.41-1.36(\mathrm{~m}, 2 \mathrm{H}), 1.35-1.26(\mathrm{~m}, 5 \mathrm{H}), 1.23-1.16$ (m, 2H), $1.08-0.98(\mathrm{~m}, 2 \mathrm{H}) \mathrm{ppm} .{ }^{13} \mathbf{C}$ NMR $\left(101 \mathrm{MHz}, \mathrm{CDCl}_{3}\right) \delta 165.7(\mathrm{~d}, J=252.2$ $\mathrm{Hz}), 165.8,155.0,132.2$ (d, $J=9.3 \mathrm{~Hz}), 126.9$ (d, $J=3.1 \mathrm{~Hz}), 115.6$ (d, $J=22.1 \mathrm{~Hz})$, 79.2, 65.3, 44.2, 36.5, 36.0, 32.3, 29.5, 28.8, 28.5, 26.6, 26.1 ppm. ${ }^{19}$ F NMR (376 MHz, $\left.\mathrm{CDCl}_{3}\right) \delta$-106.1. IR (neat, $\mathrm{cm}^{-1}$ ): 2974, 2929, 2856, 1716, 1693, 1603, 1508, 1412, 1366, 1270, 1239, 1153, 1112, 1090, 854. HRMS calcd. for $\left(\mathrm{C}_{23} \mathrm{H}_{34} \mathrm{FNNaO}_{4}\right)[\mathrm{M}+\mathrm{Na}]$ ${ }^{+}: 430.2364$ found 430.2358 .

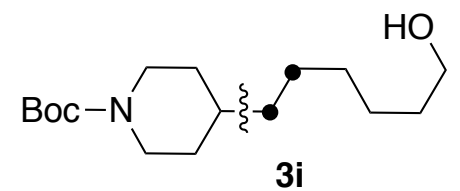

tert-butyl 4-(6-hydroxyhexyl)piperidine-1-carboxylate (3i): Following the general procedure, but using 5-hexene-1-ol (70.6 $\mu \mathrm{L}, 0.60 \mathrm{mmol})$, afforded $3 \mathbf{i}$ (35 mg, 61\% yield) as a light yellow solid. Mp $47-48^{\circ} \mathrm{C}$. In an independent experiment, $34 \mathrm{mg}(61 \%$ yield) were obtained, giving an average of $61 \%$ yield. ${ }^{1} \mathbf{H}$ NMR $\left(400 \mathrm{MHz}, \mathrm{CDCl}_{3}\right) \delta$ $4.05(\mathrm{~d}, J=13.2 \mathrm{~Hz}, 2 \mathrm{H}), 3.62(\mathrm{t}, J=6.6 \mathrm{~Hz}, 2 \mathrm{H}), 2.65(\mathrm{td}, J=12.8,2.7 \mathrm{~Hz}, 2 \mathrm{H}), 1.68$ - $1.58(\mathrm{~m}, 2 \mathrm{H}), 1.58-1.52(\mathrm{~m}, 2 \mathrm{H}), 1.44(\mathrm{~s}, 9 \mathrm{H}), 1.39-1.26(\mathrm{~m}, 8 \mathrm{H}), 1.25-1.18(\mathrm{~m}$, 2H), $1.10-0.99$ (m, 2H) ppm. ${ }^{13} \mathbf{C}$ NMR (101 MHz, $\left.\mathrm{CDCl}_{3}\right) \delta 155.1,79.3,63.1,44.2$, 36.6, 36.1, 32.9, 32.4, 29.7, 28.6, 26.7, 25.9 ppm. IR (neat, $\mathrm{cm}^{-1}$ ): 2971, 2931, 2854, $1686,1468,1408,1365,1277,1245,1171,1150,1124,1095,865$. The observed spectral data are in agreement with the ones reported in literature. ${ }^{1}$

\footnotetext{
${ }^{1}$ Bazin, H. G.; Li, Y.; Khalaf, J. K.; Mwakawari, S.; Livesay, M. T.; Evans, J. T.; Johnson, D. A.; Bioorg. Med. Chem. Lett. 2015, 25, 1318
} 


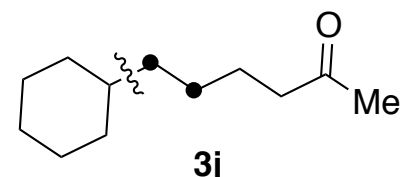

6-cyclohexylhexan-2-one (3j): Following the general procedure, but using 1cyclohexyl-2,4,6-triphenylpyridin-1-ium tetrafluoroborate (1b, $95.8 \mathrm{mg}, 0.20 \mathrm{mmol})$ and 5-hexene-2-one ( $69.3 \mu \mathrm{L}, 0.60 \mathrm{mmol})$, afforded $\mathbf{3 j}$ (19 $\mathrm{mg}, 52 \%$ yield) as a light yellow oil. In an independent experiment, $22 \mathrm{mg}$ (60\% yield) were obtained, giving an average of $56 \%$ yield. ${ }^{1} \mathbf{H}$ NMR $\left(400 \mathrm{MHz}, \mathrm{CDCl}_{3}\right) \delta 2.41(\mathrm{t}, J=7.5 \mathrm{~Hz}, 2 \mathrm{H}), 2.13(\mathrm{~s}$, $3 \mathrm{H}), 1.76-1.59(\mathrm{~m}, 5 \mathrm{H}), 1.54(\mathrm{dt}, J=14.8,7.6 \mathrm{~Hz}, 2 \mathrm{H}), 1.32-1.23(\mathrm{~m}, 3 \mathrm{H}), 1.23-$ $1.10(\mathrm{~m}, 5 \mathrm{H}), 0.95-0.72(\mathrm{~m}, 2 \mathrm{H}) \mathrm{ppm} .{ }^{13} \mathbf{C ~ N M R}\left(101 \mathrm{MHz}, \mathrm{CDCl}_{3}\right) \delta$ 209.5, 44.0, 37.6, 37.4, 33.5, 30.0, 26.9, 26.57, 26.6, 24.3 ppm. IR (neat, $\mathrm{cm}^{-1}$ ): 2920, 2850, 1715, $1448,1357,1163,962,890$. HRMS calcd. for $\left(\mathrm{C}_{12} \mathrm{H}_{22} \mathrm{NaO}\right)[\mathrm{M}+\mathrm{Na}]^{+}: 205.1563$ found 205.1558 .

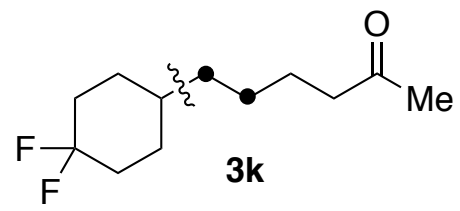

6-(4,4-difluorocyclohexyl)hexan-2-one (3k): Following the general procedure, but using 1-(4,4-difluorocyclohexyl)-2,4,6-triphenylpyridin-1-ium tetrafluoroborate (1c, $102.6 \mathrm{mg}, 0.20 \mathrm{mmol})$ and 5-hexene-2-one $(69.3 \mu \mathrm{L}, 0.60 \mathrm{mmol})$, afforded 3k (30 mg, $69 \%$ yield) as a light yellow oil. In an independent experiment, $29 \mathrm{mg}$ (67\% yield) were obtained, giving an average of $68 \%$ yield. ${ }^{1} \mathbf{H}$ NMR $\left(400 \mathrm{MHz}, \mathrm{CDCl}_{3}\right) \delta 2.42(\mathrm{t}, J=$ $7.4 \mathrm{~Hz}, 2 \mathrm{H}), 2.13$ (s, 3H), $2.10-1.99$ (m, 2H), $1.84-1.67$ (m, 3H), $1.64-1.49(\mathrm{~m}$, $3 \mathrm{H}), 1.42-1.17(\mathrm{~m}, 7 \mathrm{H})$. ppm. ${ }^{13} \mathbf{C}$ NMR $\left(101 \mathrm{MHz}, \mathrm{CDCl}_{3}\right) \delta 209.2,124.0(\mathrm{dd}, J=$ 241.8, 239.4 Hz), 43.8, 35.65 (dd, $J=5.9,2.1 \mathrm{~Hz}), 33.6(\mathrm{dd}, J=25.4,22.3 \mathrm{~Hz}), 30.0$, 29.1, 29.0, 26.8, 24.0 ppm. ${ }^{19}$ F NMR (376 MHz, $\left.\mathrm{CDCl}_{3}\right) \delta-91.55$ (d, $\left.J=234.3 \mathrm{~Hz}\right)$, $102.04(\mathrm{~d}, J=234.2 \mathrm{~Hz})$. IR (neat, $\left.\mathrm{cm}^{-1}\right): 2935,2863,1715,1449,1358,1272,1164$, 1115, 1081, 960. GC-MS: $\left(\mathrm{C}_{12} \mathrm{H}_{20} \mathrm{~F}_{2} \mathrm{O}\right)[\mathrm{M}]+$ : found $\mathrm{t}=5.492 \mathrm{~min}, \mathrm{~m} / \mathrm{z} 218.2$.<smiles>CC(C)(C)OC(=O)N1CCC(CCCc2cccc(Cl)c2)CC1</smiles>

tert-butyl 4-(3-(3-chlorophenyl)propyl)piperidine-1-carboxylate (31): Following the general procedure, but using 1-allyl-3-chlorobenzene $(87.2 \mu \mathrm{L}, 0.60 \mathrm{mmol})$, afforded 31 (42 mg, 62\% yield) as a colorless oil. In an independent experiment, $44 \mathrm{mg}$ (65\% 
yield) were obtained, giving an average of $63 \%$ yield. ${ }^{1} \mathbf{H}$ NMR $\left(400 \mathrm{MHz}, \mathrm{CDCl}_{3}\right) \delta$ $7.23-7.12(\mathrm{~m}, 3 \mathrm{H}), 7.05-7.02(\mathrm{~m}, 1 \mathrm{H}), 4.06(\mathrm{~d}, J=12.2 \mathrm{~Hz}, 2 \mathrm{H}), 2.65(\mathrm{t}, J=12.7$ $\mathrm{Hz}, 2 \mathrm{H}), 2.56(\mathrm{t}, J=7.7 \mathrm{~Hz}, 2 \mathrm{H}), 1.79-1.54(\mathrm{~m}, 4 \mathrm{H}), 1.45(\mathrm{~s}, 9 \mathrm{H}), 1.40-1.33(\mathrm{~m}$, $1 \mathrm{H}), 1.33-1.21(\mathrm{~m}, 2 \mathrm{H}), 1.13-0.99(\mathrm{~m}, 2 \mathrm{H}) \mathrm{ppm} .{ }^{13} \mathbf{C} \mathbf{N M R}\left(101 \mathrm{MHz}, \mathrm{CDCl}_{3}\right) \delta$ 155.0, 144.7, 134.1, 129.6, 128.6, 126.7, 126.0, 79.3, 44.1, 36.2, 36.0, 35.9, 32.3, 28.6, 28.4 ppm. IR (neat, $\mathrm{cm}^{-1}$ ): 2975, 2928, 2854, 1687, 1476, 1420, 1364, 1276, 1243, 1155 , 1120, 1079, 967, 867. HRMS calcd. for $\left(\mathrm{C}_{19} \mathrm{H}_{28} \mathrm{ClNNaO}_{2}\right)[\mathrm{M}+\mathrm{Na}]^{+}: 360.1701$ found 360.1705 .

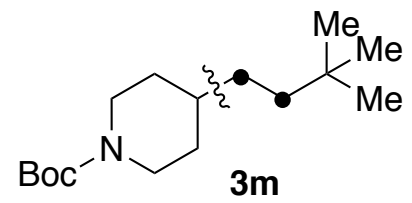

tert-butyl 4-(3,3-dimethylbutyl)piperidine-1-carboxylate $(3 \mathrm{~m})$ : Following the general procedure, but using 3,3-dimethylbut-1-ene ( $77 \mu \mathrm{L}, 0.60 \mathrm{mmol})$, afforded $3 \mathbf{m}$ (32 mg, 59\% yield) as a colorless oil. In an independent experiment, $33 \mathrm{mg}$ (61\% yield) were obtained, giving an average of $60 \%$ yield. ${ }^{1} \mathbf{H} \mathbf{~ N M R}\left(400 \mathrm{MHz}, \mathrm{CDCl}_{3}\right) \delta 4.05(\mathrm{~d}$, $J=13.1 \mathrm{~Hz}, 2 \mathrm{H}), 2.65(\mathrm{td}, J=12.8,2.7 \mathrm{~Hz}, 2 \mathrm{H}), 1.67-1.62(\mathrm{~m}, 2 \mathrm{H}), 1.44(\mathrm{~s}, 9 \mathrm{H}), 1.30$ $-1.22(\mathrm{~m}, 1 \mathrm{H}), 1.17(\mathrm{~d}, J=3.2 \mathrm{~Hz}, 4 \mathrm{H}), 1.11-1.00(\mathrm{~m}, 2 \mathrm{H}), 0.85(\mathrm{~s}, 9 \mathrm{H}) \mathrm{ppm} .{ }^{13} \mathrm{C}$ NMR $\left(101 \mathrm{MHz}, \mathrm{CDCl}_{3}\right) \delta 155.1,79.2,44.3,41.2,37.0,32.5,31.4,30.3,29.5,28.6$ ppm. IR (neat, $\mathrm{cm}^{-1}$ ): 2975, 2924, 2855, 1693, 1468, 1420, 1364, 1277, 1248, 1231, 1156, 965, 868. HRMS calcd. for $\left(\mathrm{C}_{16} \mathrm{H}_{31} \mathrm{NNaO}_{2}\right)[\mathrm{M}+\mathrm{Na}]^{+}: 292.2247$ found 292.2240

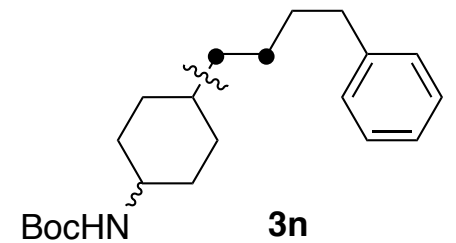

tert-butyl (4-(4-phenylbutyl)cyclohexyl)carbamate (3n): Following the general procedure, but using 1-(4-((tert-butoxycarbonyl)amino)cyclohexyl)-2,4,6triphenylpyridin-1-ium tetrafluoroborate (1d, $118.4 \mathrm{mg}, 0.20 \mathrm{mmol})$ and 4-phenyl-1butene (90 $\mu \mathrm{L}, 0.60 \mathrm{mmol})$, afforded $3 \mathrm{n}$ (34 $\mathrm{mg}, 51 \%$ yield) as a white solid. In an independent experiment, $33 \mathrm{mg}$ (50\% yield) were obtained, giving an average of $50 \%$ yield, as a 1.5:1 ratio of diastereoisomers. $\mathrm{Mp} 69-70{ }^{\circ} \mathrm{C} .{ }^{1} \mathbf{H}$ NMR $\left(400 \mathrm{MHz}, \mathrm{CDCl}_{3}\right)$ $\delta 7.32-7.23(\mathrm{~m}, 2 \mathrm{H}), 7.19-7.15(\mathrm{~m}, 3 \mathrm{H}), 4.70-4.35(\mathrm{~m}, 1 \mathrm{H}), 3.75-3.35(\mathrm{~m}, 1 \mathrm{H})$, $2.60(\mathrm{td}, J=7.7,3.8 \mathrm{~Hz}, 2 \mathrm{H}), 1.97(\mathrm{~d}, J=14.0 \mathrm{~Hz}, 1 \mathrm{H}), 1.74(\mathrm{~d}, J=12.2 \mathrm{~Hz}, 1 \mathrm{H}), 1.68$ 
- 1.49 (m, 5H), 1.45 (s, 9H), 1.39 - $1.16(\mathrm{~m}, 4 \mathrm{H}), 1.17$ - 1.08 (m, 2H), 1.08 - 0.87 (m, $2 \mathrm{H}) \mathrm{ppm} .{ }^{13} \mathbf{C} \mathbf{N M R}\left(101 \mathrm{MHz}, \mathrm{CDCl}_{3}\right) \delta 155.4,142.94,142.91,128.5,128.37,128.36$, $125.73,125.71,79.1,50.2,46.8,37.0,36.8,36.1,35.8,35.6,33.7,32.0,31.9,31.8,29.9$, 28.60, 28.58, 28.1, 26.9, 26.8 ppm. IR (neat, $\mathrm{cm}^{-1}$ ): 3354, 2980, 2923, 2848, 1680, 1517, $1497,1389,1364,1322,1240,1168,1030,978,880$. HRMS calcd. for $\left(\mathrm{C}_{21} \mathrm{H}_{33} \mathrm{NNaO}_{2}\right)$ $[\mathrm{M}+\mathrm{Na}]^{+}: 354.2404$ found 354.2407.

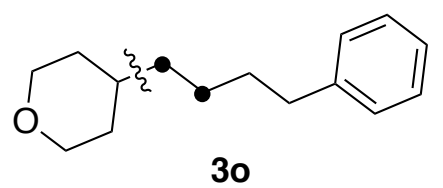

6-(tetrahydro-2H-pyran-4-yl)hexan-2-one (3o): Following the general procedure, but using 2,4,6-triphenyl-1-(tetrahydro-2H-pyran-4-yl)pyridin-1-ium tetrafluoroborate (1e, $95.8 \mathrm{mg}, 0.20 \mathrm{mmol})$ and 4-phenyl-1-butene $(90 \mu \mathrm{L}, 0.60 \mathrm{mmol})$, afforded $3 \mathbf{0}$ (30 $\mathrm{mg}, 69 \%$ yield) as a colorless oil. In an independent experiment, $30 \mathrm{mg}$ (69\% yield) were obtained, giving an average of $69 \%$ yield. ${ }^{1} \mathbf{H}$ NMR $\left(400 \mathrm{MHz}, \mathrm{CDCl}_{3}\right) \delta 7.32-$ $7.24(\mathrm{~m}, 2 \mathrm{H}), 7.21-7.17(\mathrm{~m}, 3 \mathrm{H}), 4.05-3.82(\mathrm{~m}, 2 \mathrm{H}), 3.36(\mathrm{td}, J=11.8,2.1 \mathrm{~Hz}, 2 \mathrm{H})$, $2.68-2.51(\mathrm{~m}, 2 \mathrm{H}), 1.70-1.54(\mathrm{~m}, 4 \mathrm{H}), 1.52-1.41(\mathrm{~m}, 1 \mathrm{H}), 1.39-1.32(\mathrm{~m}, 2 \mathrm{H})$, $1.31-1.21(\mathrm{~m}, 4 \mathrm{H}) \mathrm{ppm} .{ }^{13} \mathrm{C}$ NMR $\left(101 \mathrm{MHz}, \mathrm{CDCl}_{3}\right) \delta 142.8,128.5,128.4,125.8$, 68.3, 36.9, 36.1, 35.1, 33.4, 31.8, 26.1 ppm. IR (neat, $\mathrm{cm}^{-1}$ ): 2925, 2841, 1496, 1454 , 1386, 1236, 1137, 1094, 1014, 981, 856. HRMS calcd. for $\left(\mathrm{C}_{15} \mathrm{H}_{23} \mathrm{O}\right)[\mathrm{M}+\mathrm{H}]^{+}$: 219.1743 found 219.1746 .

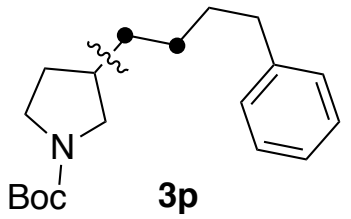

tert-butyl-3-(4-phenylbutyl)pyrrolidine-1-carboxylate (3p): Following the general procedure, but using 1-(1-(tert-butoxycarbonyl)pyrrolidin-3-yl)-2,4,6triphenylpyridin-1-ium tetrafluoroborate (1f, $112.8 \mathrm{mg}, 0.20 \mathrm{mmol}$ ) and 4-phenyl-1butene ( $90 \mu \mathrm{L}, 0.60 \mathrm{mmol}$ ), afforded $3 \mathbf{p}$ (46 mg, 76\% yield) as a colorless oil. In an independent experiment, $46 \mathrm{mg}$ (76\% yield) were obtained, giving an average of $76 \%$ yield. ${ }^{1} \mathbf{H}$ NMR $\left(400 \mathrm{MHz}, \mathrm{CDCl}_{3}\right) \delta 7.32-7.23(\mathrm{~m}, 2 \mathrm{H}), 7.18(\mathrm{t}, J=6.4 \mathrm{~Hz}, 3 \mathrm{H}), 3.57$ -7.23 (m, 2H), $3.28-3.17(\mathrm{~m}, 1 \mathrm{H}), 2.84$ (dt, $J=19.9,9.7 \mathrm{~Hz}, 1 \mathrm{H}), 2.61$ (dt, $J=7.9$, $2.8 \mathrm{~Hz}, 2 \mathrm{H}), 2.13-2.04(\mathrm{~m}, 1 \mathrm{H}), 1.98-1.91(\mathrm{~m}, 1 \mathrm{H}), 1.63$ (dt, $J=11.2,3.6 \mathrm{~Hz}, 2 \mathrm{H})$, $1.46(\mathrm{~s}, 9 \mathrm{H}), 1.44-1.31(\mathrm{~m}, 5 \mathrm{H}) \mathrm{ppm} .{ }^{13} \mathrm{C}$ NMR $\left(101 \mathrm{MHz}, \mathrm{CDCl}_{3}\right) \delta$ 154.7, 142.6, 
$128.5,128.4,125.8,79.0,51.8,51.4,45.9,45.6,39.2,38.4,35.9,33.2,32.0,31.6,31.3$, 28.7, 27.9 ppm. IR (neat, $\mathrm{cm}^{-1}$ ): 2974, 2929, 2857, 1692, 1454, 1400, 1365, 1168, 1121 , 910, 881. HRMS calcd. for $\left(\mathrm{C}_{19} \mathrm{H}_{29} \mathrm{NNaO}_{2}\right)[\mathrm{M}+\mathrm{Na}]^{+}: 326.2090$ found 326.2088.

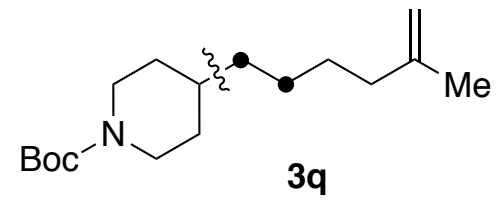

tert-butyl 4-(5-methylhex-5-en-1-yl)piperidine-1-carboxylate (3q): Following the general procedure, but using 2-methylhexa-1,5-diene $(80.9 \mu \mathrm{L}, 0.60 \mathrm{mmol})$, afforded 3q (38 mg, 68\% yield) as a colorless oil. In an independent experiment, $40 \mathrm{mg}(70 \%$ yield) were obtained, giving an average of $69 \%$ yield. ${ }^{1} \mathbf{H}$ NMR $\left(400 \mathrm{MHz}, \mathrm{CDCl}_{3}\right) \delta$ $4.79-4.53(\mathrm{~m}, 2 \mathrm{H}), 4.05(\mathrm{~s}, 2 \mathrm{H}), 2.65(\mathrm{t}, J=12.8 \mathrm{~Hz}, 2 \mathrm{H}), 2.07-1.88(\mathrm{~m}, 2 \mathrm{H}), 1.70$ (s, 3H), $1.66-1.57(\mathrm{~m}, 2 \mathrm{H}), 1.44(\mathrm{~s}, 9 \mathrm{H}), 1.42-1.35(\mathrm{~m}, 2 \mathrm{H}), 1.34-1.16(\mathrm{~m}, 5 \mathrm{H})$, $1.10-1.00(\mathrm{~m}, 2 \mathrm{H}) \mathrm{ppm} .{ }^{13} \mathrm{C}$ NMR $\left(101 \mathrm{MHz}, \mathrm{CDCl}_{3}\right) \delta 155.1,146.2,109.8,79.2$, 44.2, 37.9, 36.6, 36.1, 32.4, 28.6, 27.9, 26.4, 22.5 ppm. IR (neat, $\mathrm{cm}^{-1}$ ): 2974, 2927, 2853, 1692, 1419, 1364, 1277, 1240, 1155, 970, 884. HRMS calcd. for $\left(\mathrm{C}_{17} \mathrm{H}_{31} \mathrm{NNaO}_{2}\right)$ $[\mathrm{M}+\mathrm{Na}]^{+}: 304.2247$ found 304.2248.

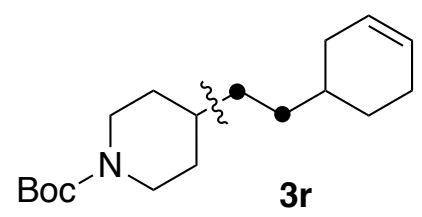

tert-butyl 4-(2-(cyclohex-3-en-1-yl)ethyl)piperidine-1-carboxylate (3r): Following the general procedure, but using 4-vinylcyclohex-1-ene (78 $\mu \mathrm{L}, 0.60 \mathrm{mmol})$, afforded 3r (27 mg, 46\% yield) as a colorless oil. In an independent experiment, $29 \mathrm{mg}(50 \%$ yield) were obtained, giving an average of $48 \%$ yield. ${ }^{1} \mathbf{H}$ NMR $\left(400 \mathrm{MHz}, \mathrm{CDCl}_{3}\right) \delta$ $5.77-5.40(\mathrm{~m}, 2 \mathrm{H}), 4.06(\mathrm{~d}, J=13.2 \mathrm{~Hz}, 2 \mathrm{H}), 2.66(\mathrm{td}, J=13.2 \mathrm{~Hz}, 2.4 \mathrm{~Hz}, 2 \mathrm{H}), 2.22$ - $1.91(\mathrm{~m}, 3 \mathrm{H}), 1.81-1.53(\mathrm{~m}, 4 \mathrm{H}), 1.45(\mathrm{~s}, 9 \mathrm{H}), 1.52$ - $1.42(\mathrm{~m}, 1 \mathrm{H}), 1.40-1.14(\mathrm{~m}$, $6 \mathrm{H}), 1.12-1.01(\mathrm{~m}, 2 \mathrm{H}) \mathrm{ppm} .{ }^{13} \mathbf{C}$ NMR $\left(101 \mathrm{MHz}, \mathrm{CDCl}_{3}\right) \delta 155.1,127.2,126.7$, 79.3, 44.2, 36.4, 33.9, 33.8, 33.7, 32.4, 32.1, 29.1, 28.6, 25.4 ppm. IR (neat, $\mathrm{cm}^{-1}$ ): 2974, 2915, 2849, 1691, 1420, 1364, 1276, 1239, 1157, 1127, 967, 868. HRMS calcd. for $\left(\mathrm{C}_{18} \mathrm{H}_{31} \mathrm{NNaO}_{2}\right)[\mathrm{M}+\mathrm{Na}]^{+}: 316.2247$ found 316.2258 . 


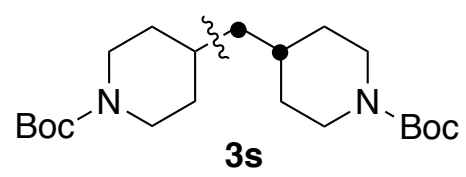

di-tert-butyl 4,4'-methylenebis(piperidine-1-carboxylate) (3s): Following the general procedure, but using $\mathrm{NiI}_{2}(6.2 \mathrm{mg}, 10 \mathrm{~mol} \%)$, 2-(6-methylpyridin-2-yl)-4,5dihydrooxazole (L6, $6.4 \mathrm{mg}, 20 \mathrm{~mol} \%$ ), $\mathrm{Na}_{2} \mathrm{HPO}_{4}$ (113.6 mg, $0.80 \mathrm{mmol}$ ), (EtO) ${ }_{3} \mathrm{SiH}$ (74 $\mu \mathrm{L}, 0.40 \mathrm{mmol}$ ) and tert-butyl 4-methylenepiperidine-1-carboxylate (2o, $122 \mathrm{ul}$, $0.60 \mathrm{mmol})$, in DMSO and 1.4-dioxane $(3: 1,1.2 \mathrm{~mL})$ at $35^{\circ} \mathrm{C}$ stirring $24 \mathrm{~h}$, afforded 3s (46 mg, 60\% yield) as a light yellow solid. Mp $90-91{ }^{\circ} \mathrm{C}$. In an independent experiment, $46 \mathrm{mg}$ (60\% yield) were obtained, giving an average of $60 \%$ yield. ${ }^{1} \mathbf{H}$ NMR $\left(400 \mathrm{MHz}, \mathrm{CDCl}_{3}\right) \delta 4.05$ (brs, 4H), 2.65 (t, $\left.J=12.8 \mathrm{~Hz}, 4 \mathrm{H}\right), 1.68-1.56$ (m, 4H), $1.52-1.47(\mathrm{~m}, 2 \mathrm{H}), 1.44(\mathrm{~s}, 18 \mathrm{H}), 1.14(\mathrm{t}, J=7.0 \mathrm{~Hz}, 2 \mathrm{H}), 1.11-0.92(\mathrm{~m}, 4 \mathrm{H})$ ppm. ${ }^{13} \mathbf{C}$ NMR $\left(101 \mathrm{MHz}, \mathrm{CDCl}_{3}\right) \delta 155.0,79.3,44.1,43.6,32.7,32.5,28.6$ ppm. IR (neat, $\mathrm{cm}^{-1}$ ): 2972, 2910, 2845, 1684, 1142, 1364, 1279, 1229, 1165, 1137, 1091, 976, 863. HRMS calcd. for $\left(\mathrm{C}_{21} \mathrm{H}_{38} \mathrm{~N}_{2} \mathrm{NaO}_{4}\right)[\mathrm{M}+\mathrm{Na}]^{+}: 405.2724$ found 405.2712.

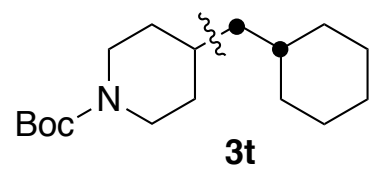

tert-butyl 4-(cyclohexylmethyl)piperidine-1-carboxylate (3t): Following the general procedure $\mathrm{B}$, but using $\mathrm{NiI}_{2}$ (6.2 $\mathrm{mg}, 10 \mathrm{~mol} \%$ ), 2-(6-methylpyridin-2-yl)-4,5dihydrooxazole (L6, $6.4 \mathrm{mg}, 20 \mathrm{~mol} \%$ ), $\mathrm{Na}_{2} \mathrm{HPO}_{4}$ (113.6 mg, $0.80 \mathrm{mmol}$ ), (EtO) ${ }_{3} \mathrm{SiH}$ (74 $\mu \mathrm{L}, 0.40 \mathrm{mmol}, 3.0$ equiv) and methylenecyclohexane $(72.1 \mu \mathrm{L}, 0.60 \mathrm{mmol}$ ), in DMSO and 1.4-dioxane (3:1, $1.2 \mathrm{~mL})$ at $35{ }^{\circ} \mathrm{C}$ stirring $24 \mathrm{~h}$, afforded $3 \mathrm{t}$ (33 $\mathrm{mg}, 59 \%$ yield) as a white solid. Mp $47-48{ }^{\circ} \mathrm{C}$. In an independent experiment, $33 \mathrm{mg}(59 \%$ yield) were obtained, giving an average of $59 \%$ yield. ${ }^{1} \mathbf{H} \mathbf{~ N M R}\left(400 \mathrm{MHz}, \mathrm{CDCl}_{3}\right) \delta$ $4.05(\mathrm{~d}, J=13.2 \mathrm{~Hz}, 2 \mathrm{H}), 2.66(\mathrm{td}, J=12.9,2.7 \mathrm{~Hz}, 2 \mathrm{H}), 1.75-1.55(\mathrm{~m}, 7 \mathrm{H}), 1.44$ (s, 9H), $1.37-1.12(\mathrm{~m}, 5 \mathrm{H}), 1.12-0.96(\mathrm{~m}, 4 \mathrm{H}), 0.89-0.79(\mathrm{~m}, 2 \mathrm{H}) \mathrm{ppm} .{ }^{13} \mathbf{C}$ NMR $\left(101 \mathrm{MHz}, \mathrm{CDCl}_{3}\right) \delta 155.1,79.2,44.6,44.2,34.3,33.8,32.9,32.7,28.6,26.8,26.5$ ppm. IR (neat, $\mathrm{cm}^{-1}$ ): 2980, 2917, 2848, 1448, 1679, 1447, 1413, 1366, 1281, 1233, 1161, 1118, 1009, 974, 866. HRMS calcd. for $\left(\mathrm{C}_{17} \mathrm{H}_{31} \mathrm{NNaO}_{2}\right)[\mathrm{M}+\mathrm{Na}]^{+}: 304.2247$ found 304.2246. 


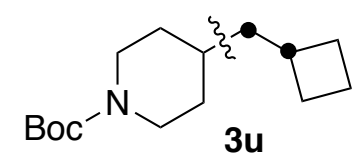

tert-butyl 4-(cyclobutylmethyl)piperidine-1-carboxylate (3u): Following the general procedure B, but using $\mathrm{NiI}_{2}$ (6.2 mg, $10 \mathrm{~mol} \%$ ), 2-(6-methylpyridin-2-yl)-4,5dihydrooxazole (L6, $6.4 \mathrm{mg}, 20 \mathrm{~mol} \%$ ), $\mathrm{Na}_{2} \mathrm{HPO}_{4}$ (113.6 mg, $0.80 \mathrm{mmol}$ ), (EtO) $)_{3} \mathrm{SiH}$ (74 $\mu \mathrm{L}, 0.40 \mathrm{mmol}, 3.0$ equiv) and methylenecyclobutane $(55.5 \mu \mathrm{L}, 0.60 \mathrm{mmol})$, in DMSO and 1.4-dioxane $(3: 1,1.2 \mathrm{~mL})$ at $35^{\circ} \mathrm{C}$ stirring $24 \mathrm{~h}$, afforded $3 \mathbf{u}(32 \mathrm{mg}, 62 \%$ yield) as a colorless oil. In an independent experiment, $30 \mathrm{mg}$ (60\% yield) were obtained, giving an average of $61 \%$ yield. ${ }^{1} \mathbf{H}$ NMR $\left(400 \mathrm{MHz}, \mathrm{CDCl}_{3}\right) \delta 4.03(\mathrm{~d}, J=$ $13.0 \mathrm{~Hz}, 2 \mathrm{H}), 2.64(\mathrm{td}, J=12.9,2.8 \mathrm{~Hz}, 2 \mathrm{H}), 2.35$ (m, 1H), $2.05-1.98(\mathrm{~m}, 2 \mathrm{H}), 1.87$ - $1.73(\mathrm{~m}, 2 \mathrm{H}), 1.66-1.51(\mathrm{~m}, 4 \mathrm{H}), 1.44(\mathrm{~s}, 9 \mathrm{H}), 1.38-1.20(\mathrm{~m}, 3 \mathrm{H}), 1.16-0.93(\mathrm{~m}$, $2 \mathrm{H}) \mathrm{ppm} .{ }^{13} \mathrm{C}$ NMR $\left(101 \mathrm{MHz}, \mathrm{CDCl}_{3}\right) \delta 142.7,128.4,128.3,125.6,68.2,36.8,36.0$, 35.0, 33.3, 31.6, 26.0 ppm. IR (neat, $\mathrm{cm}^{-1}$ ): 2974, 2927, 2850, 1691, 1419, 1364, 1277 , 1245, 1164, 1145, 972, 867. HRMS calcd. for $\left(\mathrm{C}_{15} \mathrm{H}_{27} \mathrm{NNaO}_{2}\right)[\mathrm{M}+\mathrm{Na}]^{+}: 276.1934$ found 276.1945 .

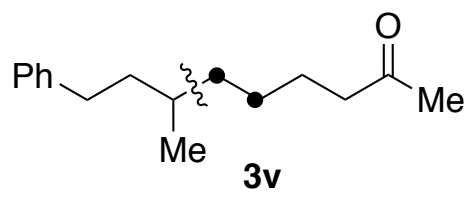

7-methyl-9-phenylnonan-2-one (3v): Following the general procedure, but using 2,4,6-triphenyl-1-(4-phenylbutan-2-yl)pyridin-1-ium tetrafluoroborate (1g, $210.8 \mathrm{mg}$, $0.40 \mathrm{mmol}$ ) and 5-hexene-2-one (23.1 $\mu \mathrm{L}, 0.20 \mathrm{mmol})$, afforded 3v (19 mg, 41\% yield) as a light yellow oil. In an independent experiment, $21 \mathrm{mg}$ (45\% yield) were obtained, giving an average of $43 \%$ yield. ${ }^{1} \mathbf{H}$ NMR $\left(300 \mathrm{MHz}, \mathrm{CDCl}_{3}\right) \delta 7.32-7.24(\mathrm{~m}, 2 \mathrm{H})$, $7.21-7.12(\mathrm{~m}, 3 \mathrm{H}), 2.75-2.50(\mathrm{~m}, 2 \mathrm{H}), 2.42$ (t, $J=7.4 \mathrm{~Hz}, 2 \mathrm{H}), 2.13(\mathrm{~s}, 3 \mathrm{H}), 1.79-$ $1.04(\mathrm{~m}, 9 \mathrm{H}), 0.92(\mathrm{~d}, J=6.2 \mathrm{~Hz}, 3 \mathrm{H}) \mathrm{ppm} .{ }^{13} \mathbf{C} \mathbf{N M R}\left(75 \mathrm{MHz}, \mathrm{CDCl}_{3}\right) \delta 209.4,143.2$, $128.5,128.4,125.7,43.9,39.0,36.8,33.6,32.5,30.0,26.7,24.3,19.7$ ppm. IR (neat, $\left.\mathrm{cm}^{-1}\right)$ : 2977, 2928, 2858, 1715, 1454, 1357, 1164, 1030, 957. HRMS calcd. for $\left(\mathrm{C}_{16} \mathrm{H}_{24} \mathrm{NaO}\right)[\mathrm{M}+\mathrm{Na}]^{+}: 255.1719$ found 255.1724 . 


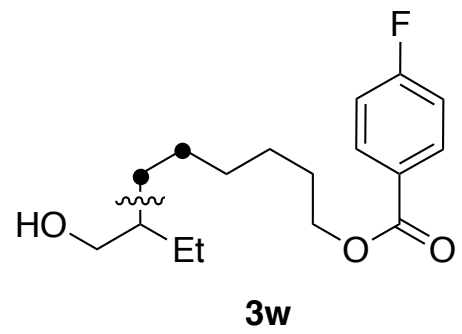

7-(hydroxymethyl)nonyl 4-fluorobenzoate (3w): Following the general procedure, but using 1-(1-hydroxybutan-2-yl)-2,4,6-triphenylpyridin-1-ium tetrafluoroborate (1h), $93.4 \mathrm{mg}, 0.20 \mathrm{mmol}$ ) and hex-5-en-1-yl 4-fluorobenzoate (133.2 $\mathrm{mg}, 0.60 \mathrm{mmol}$ ), afforded 3w (30 mg, 51\% yield) as a colorless oil. In an independent experiment, 29 $\mathrm{mg}\left(49 \%\right.$ yield) were obtained, giving an average of 50\% yield. ${ }^{1} \mathbf{H}$ NMR (400 MHz, $\left.\mathrm{CDCl}_{3}\right) \delta 8.05(\mathrm{dd}, J=8.9,5.5 \mathrm{~Hz}, 2 \mathrm{H}), 7.10(\mathrm{t}, J=8.7 \mathrm{~Hz}, 2 \mathrm{H}), 4.30(\mathrm{t}, J=6.7 \mathrm{~Hz}$, 2H), $3.54(\mathrm{~d}, J=5.1 \mathrm{~Hz}, 2 \mathrm{H}), 1.83-1.66(\mathrm{~m}, 2 \mathrm{H}), 1.54-1.06(\mathrm{~m}, 12 \mathrm{H}), 0.89(\mathrm{t}, J=$ $7.3 \mathrm{~Hz}, 3 \mathrm{H}) \mathrm{ppm} .{ }^{13} \mathbf{C}$ NMR $\left(101 \mathrm{MHz}, \mathrm{CDCl}_{3}\right) \delta 165.9,165.8(\mathrm{~d}, J=254.4 \mathrm{~Hz}), 132.2$ $(\mathrm{d}, J=9.3 \mathrm{~Hz}), 126.9$ (d, $J=2.8 \mathrm{~Hz}), 115.6$ (d, $J=22.0 \mathrm{~Hz}), 65.38,65.37,42.1,30.5$, 29.8, 28.8, 26.9, 26.1, 23.5, 11.3 ppm. ${ }^{19} \mathrm{~F}$ NMR (376 MHz, $\left.\mathrm{CDCl}_{3}\right) \delta-106.12(\mathrm{tt}, J=$ 8.5, $5.4 \mathrm{~Hz}$ ). IR (neat, $\mathrm{cm}^{-1}$ ): 3436, 2975, 2928, 2858, 1718, 1603, 1508, 1462, 1270 , $1238,1153,1112,1090,1037,854$. HRMS calcd. for $\left(\mathrm{C}_{17} \mathrm{H}_{25} \mathrm{FNaO}_{3}\right)[\mathrm{M}+\mathrm{Na}]^{+}$: 319.1680 found 319.1682 . 


\section{Ni-Catalyzed Deaminative Alkylation with Internal Olefins (Table 3)}

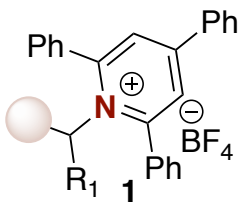

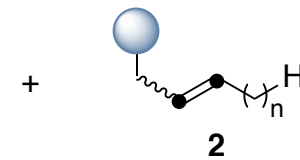

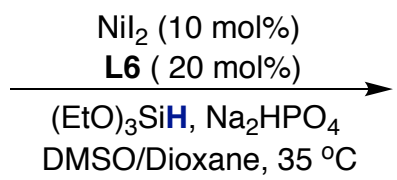

General procedure B: An oven-dried $8 \mathrm{~mL}$ screw-cap test tube containing a stirring bar was charged with $\mathrm{NiI}_{2}(6.2 \mathrm{mg}, 10 \mathrm{~mol} \%)$, 2-(6-methylpyridin-2-yl)-4,5-dihydrooxazole (L6, $6.4 \mathrm{mg}, 20 \mathrm{~mol} \%), \mathrm{Na}_{2} \mathrm{HPO}_{4}(113.6 \mathrm{mg}, 0.80 \mathrm{mmol})$ and pyridinium salt $(\mathbf{1}, 0.20$ mmol). Subsequently, the tube was sealed with a Teflon-lined screw cap, then evacuated and back-filled with $\mathrm{Ar}$ (3 times). Afterwards, olefin (2, $0.60 \mathrm{mmol}),(\mathrm{EtO})_{3} \mathrm{SiH}(74 \mu \mathrm{L}$, $0.40 \mathrm{mmol}, 2.0$ equiv), DMSO and 1.4-dioxane (3:1, $1.2 \mathrm{~mL})$ were added via syringe. Then, the tube was stirred at $35{ }^{\circ} \mathrm{C}$ for $24 \mathrm{~h}$. After the reaction was completed, the mixture was diluted with EtOAc, filtered through silica gel and concentrated under vacuum. The corresponding product was purified by column chromatography on silica gel. Note: if the corresponding product possesses a similar $R_{f}$ to that of 2,4,6-triphenylpyridine, then toluene should be used as eluent followed by hexane/EtOAc mixtures.

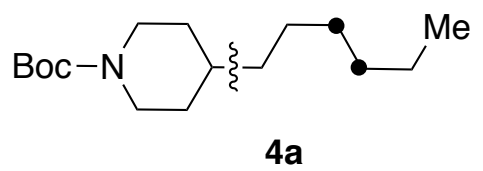

tert-butyl 4-hexylpiperidine-1-carboxylate (4a): Following the general procedure B, but using trans-3-hexene (2r, $74.5 \mu \mathrm{L}, 0.60 \mathrm{mmol})$, afforded $4 \mathbf{a}(32 \mathrm{mg}, 59 \%$ yield) as a colorless oil. In an independent experiment, $31 \mathrm{mg}$ (57\% yield) were obtained, giving an average of 58\% yield, 99:1 ratio. ${ }^{1} \mathbf{H} \mathbf{N M R}\left(400 \mathrm{MHz}, \mathrm{CDCl}_{3}\right) \delta 4.05$ (brs, 2H), 2.65 (t, $J=12.7 \mathrm{~Hz}, 2 \mathrm{H}), 1.62(\mathrm{~d}, J=12.5 \mathrm{~Hz}, 2 \mathrm{H}), 1.44(\mathrm{~s}, 9 \mathrm{H}), 1.42-1.16(\mathrm{~m}, 11 \mathrm{H}), 1.10$ $-0.99(\mathrm{~m}, 2 \mathrm{H}), 0.87(\mathrm{t}, J=6.8 \mathrm{~Hz}, 3 \mathrm{H}) \mathrm{ppm} .{ }^{13} \mathbf{C ~ N M R}\left(101 \mathrm{MHz}, \mathrm{CDCl}_{3}\right) \delta 155.1$, 79.2, 44.2, 36.7, 36.1, 32.4, 32.0, 29.6, 28.6, 26.7, 22.8, 14.2 ppm. IR (neat, $\mathrm{cm}^{-1}$ ): 2957, 2923, 2853, 1693, 1449, 1419, 1365, 1277, 1238, 1169, 1149, 1097, 968, 867. HRMS calcd. for $\left(\mathrm{C}_{16} \mathrm{H}_{31} \mathrm{NNaO}_{2}\right)[\mathrm{M}+\mathrm{Na}]^{+}: 292.2247$ found 292.2240. 


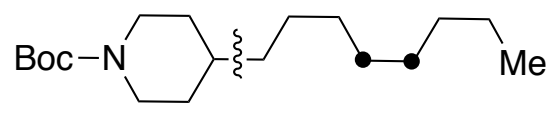

$4 b$

tert-butyl 4-octylpiperidine-1-carboxylate (4b): Following the general procedure B, but using trans-4-octene ( $2 \mathrm{~s}, 94.1 \mu \mathrm{L}, 0.60 \mathrm{mmol})$, afforded $\mathbf{4 b}$ (40 mg, 67\% yield) as a colorless oil. In an independent experiment, $37 \mathrm{mg}$ (63\% yield) were obtained, giving an average of 65\% yield, 39:1 ratio. ${ }^{1} \mathbf{H} \mathbf{N M R}\left(400 \mathrm{MHz}, \mathrm{CDCl}_{3}\right) \delta 4.05$ (brs, 2H), 2.65 $(\mathrm{t}, J=12.9 \mathrm{~Hz}, 2 \mathrm{H}), 1.66-1.54(\mathrm{~m}, 2 \mathrm{H}), 1.44(\mathrm{~s}, 9 \mathrm{H}), 1.38-1.16(\mathrm{~m}, 15 \mathrm{H}), 1.12-$ $0.97(\mathrm{~m}, 2 \mathrm{H}), 0.87(\mathrm{t}, J=6.8 \mathrm{~Hz}, 3 \mathrm{H}) \mathrm{ppm} .{ }^{13} \mathbf{C} \mathbf{N M R}\left(101 \mathrm{MHz}, \mathrm{CDCl}_{3}\right) \delta 155.1,79.2$, $44.3,36.7,36.1,32.4,32.0,30.0,29.7,29.5,28.6,26.8,22.8,14.2$ ppm. IR (neat, cm $\left.{ }^{1}\right):$ 2971, 2922, 2852, 1694, 1449, 1419, 1364, 1277, 1242, 1164, 1101, 964, 869. HRMS calcd. for $\left(\mathrm{C}_{18} \mathrm{H}_{35} \mathrm{NNaO}_{2}\right)[\mathrm{M}+\mathrm{Na}]^{+}: 320.2560$ found 320.2562 .

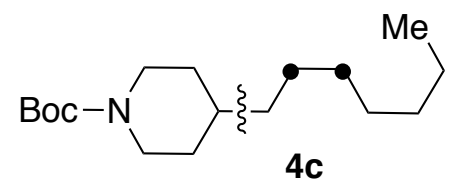

tert-butyl 4-heptylpiperidine-1-carboxylate (4c): Following the general procedure B, but using trans-4-heptene $(\mathbf{2 t}, 84 \mu \mathrm{L}, 0.60 \mathrm{mmol})$, afforded $\mathbf{4 c}(33 \mathrm{mg}, 58 \%$ yield) as a colorless oil. In an independent experiment, $32 \mathrm{mg}$ (56\% yield) were obtained, giving an average of 57\% yield, 36:1 ratio. ${ }^{1} \mathbf{H} \mathbf{N M R}\left(400 \mathrm{MHz}, \mathrm{CDCl}_{3}\right) \delta 4.05$ (brs, $2 \mathrm{H}$ ), 2.66 $(\mathrm{t}, J=12.5 \mathrm{~Hz}, 2 \mathrm{H}), 1.68-1.60(\mathrm{~m}, 2 \mathrm{H}), 1.44(\mathrm{~s}, 9 \mathrm{H}), 1.38-1.13(\mathrm{~m}, 13 \mathrm{H}), 1.10-$ $0.99(\mathrm{~m}, 2 \mathrm{H}), 0.87(\mathrm{t}, J=6.8 \mathrm{~Hz}, 3 \mathrm{H}) \mathrm{ppm} .{ }^{13} \mathbf{C ~ N M R}\left(101 \mathrm{MHz}, \mathrm{CDCl}_{3}\right) \delta 155.1,79.2$, 44.3, 36.7, 36.1, 32.4, 32.0, 29.9, 29.5, 28.6, 26.8, 22.8, 14.2 ppm. IR (neat, $\mathrm{cm}^{-1}$ ): 2975, 2923, 2852, 1694, 1449, 1419, 1365, 1277, 1245, 1168, 1099, 970, 868. HRMS calcd. for $\left(\mathrm{C}_{17} \mathrm{H}_{33} \mathrm{NNaO}_{2}\right)[\mathrm{M}+\mathrm{Na}]^{+}: 306.2404$ found 306.2404.

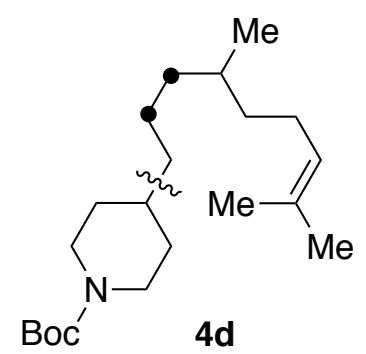

tert-butyl 4-(4,8-dimethylnon-7-en-1-yl)piperidine-1-carboxylate (4d): Following the general procedure B, but using 2,6-dimethylnona-2,7-diene (2w, $99.6 \mathrm{mg}, 0.60$ mmol), afforded $\mathbf{4 d}$ (34 mg, 50\% yield) as a colorless oil. In an independent experiment, $31 \mathrm{mg}$ (46\% yield) were obtained, giving an average of $48 \%$ yield, $54: 1$ ratio. ${ }^{1} \mathbf{H}$ NMR 
$\left(400 \mathrm{MHz}, \mathrm{CDCl}_{3}\right) \delta 5.23-4.97(\mathrm{~m}, 1 \mathrm{H}), 4.05$ (brs, 2H), $2.66(\mathrm{t}, J=12.7 \mathrm{~Hz}, 2 \mathrm{H}), 2.06$ - $1.87(\mathrm{~m}, 2 \mathrm{H}), 1.74-1.56(\mathrm{~m}, 7 \mathrm{H}), 1.45(\mathrm{~s}, 9 \mathrm{H}), 1.41-1.16(\mathrm{~m}, 9 \mathrm{H}), 1.15-1.01$ (m, $4 \mathrm{H}), 0.85(\mathrm{~d}, J=6.5 \mathrm{~Hz}, 3 \mathrm{H}) \mathrm{ppm} .{ }^{13} \mathbf{C}$ NMR $\left(101 \mathrm{MHz}, \mathrm{CDCl}_{3}\right) \delta 155.1,131.1,125.2$, 79.2, 44.3, 37.3, 37.0, 36.2, 32.5, 32.42, 32.37, 28.6, 25.9, 25.7, 24.1, 19.7, 17.8 ppm. IR (neat, $\mathrm{cm}^{-1}$ ): 3356, 2977, 2924, 2851, 1694, 1421, 1365, 1277, 1244, 1161, 968, 867. HRMS calcd. for $\left(\mathrm{C}_{21} \mathrm{H}_{39} \mathrm{NNaO}_{2}\right)[\mathrm{M}+\mathrm{Na}]^{+}: 360.2873$ found 360.2873 .

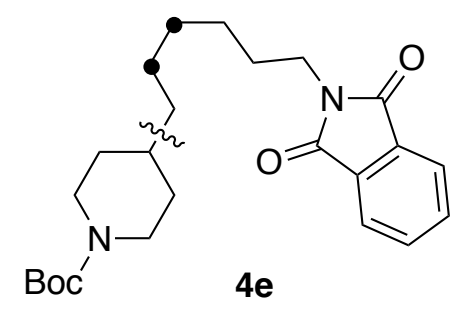

tert-butyl 4-(6-(1,3-dioxoisoindolin-2-yl)hexyl)piperidine-1-carboxylate (4e):

Following the general procedure B, but using 2-(hex-4-en-1-yl)isoindoline-1,3-dione (2x, $137.4 \mathrm{mg}, 0.60 \mathrm{mmol})$, afforded $4 \mathbf{e}$ (42 mg, 51\% yield) as a colorless oil. In an independent experiment, $41 \mathrm{mg}$ (50\% yield) were obtained, giving an average of $50 \%$ yield, 40:1 ratio. ${ }^{1} \mathbf{H} \mathbf{N M R}\left(400 \mathrm{MHz}, \mathrm{CDCl}_{3}\right) \delta 7.82(\mathrm{dd}, J=5.4,3.0 \mathrm{~Hz}, 2 \mathrm{H}), 7.69(\mathrm{dd}$, $J=5.5,3.0 \mathrm{~Hz}, 2 \mathrm{H}), 4.03$ (brs, 2H), 3.65 (t, $J=7.8 \mathrm{~Hz}, 2 \mathrm{H}), 2.63(\mathrm{t}, J=12.7 \mathrm{~Hz}, 2 \mathrm{H})$, $1.71-1.54(\mathrm{~m}, 4 \mathrm{H}), 1.43(\mathrm{~s}, 9 \mathrm{H}), 1.35-1.23(\mathrm{~m}, 7 \mathrm{H}), 1.22-1.15(\mathrm{~m}, 2 \mathrm{H}), 1.07-0.97$ $(\mathrm{m}, 2 \mathrm{H}) \mathrm{ppm} .{ }^{13} \mathbf{C} \mathbf{N M R}\left(101 \mathrm{MHz}, \mathrm{CDCl}_{3}\right) \delta 168.5,155.0,134.0,132.3,123.2,79.2$, 44.2, 38.1, 36.5, 36.0, 32.3, 29.4, 28.6, 28.6, 26.9, 26.5 ppm. IR (neat, $\mathrm{cm}^{-1}$ ): 2974, 2926, 2854, 1772, 1710, 1687, 1421, 1394, 1364, 1238, 1161, 1087, 964, 867. HRMS calcd. for $\left(\mathrm{C}_{24} \mathrm{H}_{34} \mathrm{~N}_{2} \mathrm{NaO}_{4}\right)[\mathrm{M}+\mathrm{Na}]^{+}: 437.2411$ found 437.2415 .

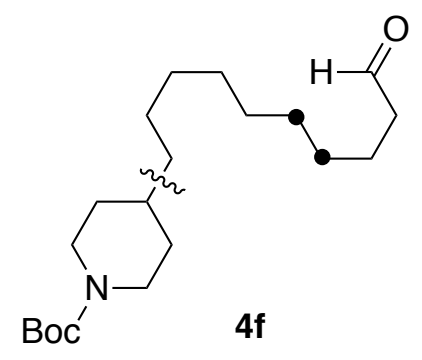

tert-butyl 4-(4,8-dimethylnon-7-en-1-yl)piperidine-1-carboxylate (4f): Following the general procedure B, but using 4-decenal (2y, $108 \mu \mathrm{L}, 0.60 \mathrm{mmol})$, afforded $\mathbf{4 f}$ (35 $\mathrm{mg}, 52 \%$ yield) as a light yellow oil. In an independent experiment, $37 \mathrm{mg}$ (54\% yield) were obtained, giving an average of 53\% yield, 29:1 ratio. ${ }^{1} \mathbf{H} \mathbf{~ N M R}\left(400 \mathrm{MHz}, \mathrm{CDCl}_{3}\right)$ $\delta 4.10(\mathrm{t}, J=7.1 \mathrm{~Hz}, 2 \mathrm{H}), 2.75(\mathrm{t}, J=12.7 \mathrm{~Hz}, 2 \mathrm{H}), 2.51-2.28(\mathrm{~m}, 3 \mathrm{H}), 1.76(\mathrm{t}, J=$ $13.5 \mathrm{~Hz}, 2 \mathrm{H}), 1.59-1.50(\mathrm{~m}, 3 \mathrm{H}), 1.50-1.47(\mathrm{~m}, 1 \mathrm{H}), 1.44(\mathrm{~s}, 9 \mathrm{H}), 1.31-1.20(\mathrm{~m}$, 
$12 \mathrm{H}), 0.86(\mathrm{t}, J=6.8 \mathrm{~Hz}, 3 \mathrm{H}) \mathrm{ppm} .{ }^{13} \mathbf{C} \mathbf{N M R}\left(101 \mathrm{MHz}, \mathrm{CDCl}_{3}\right) \delta 212.6,154.8,79.7$, $48.7,43.4,40.7,32.0,29.5,29.41,29.37,28.6,27.7,23.8,22.8,14.2$ ppm. IR (neat, $\left.\mathrm{cm}^{-1}\right): 2974,2924,2854,1692,1448,1420,1365,1277,1234,1166,1132,1013,976$, 866. HRMS calcd. for $\left(\mathrm{C}_{20} \mathrm{H}_{37} \mathrm{NNaO}_{3}\right)[\mathrm{M}+\mathrm{Na}]^{+}: 362.2666$ found 362.2661.

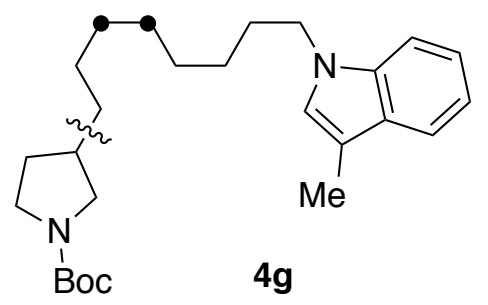

tert-butyl-3-(8-(3-methyl-1H-indol-1-yl)octyl)pyrrolidine-1-carboxylate

(4g):

Following the general procedure B, but using 1-(1-(tert-butoxycarbonyl)pyrrolidin-3yl)-2,4,6-triphenylpyridin-1-ium tetrafluoroborate (1f, $112.8 \mathrm{mg}, 0.20 \mathrm{mmol}$ ) and 3methyl-1-(oct-5-en-1-yl)-1H-indole (144.6 mg, $0.60 \mathrm{mmol}$ ), afforded $4 \mathrm{~g}$ (45 mg, 55\% yield) as a colorless oil. In an independent experiment, $45 \mathrm{mg}$ (55\% yield) were obtained, giving an average of 55\% yield, 99:1 ratio. ${ }^{1} \mathbf{H} \mathbf{N M R}\left(400 \mathrm{MHz}, \mathrm{CDCl}_{3}\right) \delta$ 7.57 (dt, $J=7.9,1.0 \mathrm{~Hz}, 1 \mathrm{H}), 7.29$ (dt, $J=8.2,0.9 \mathrm{~Hz}, 1 \mathrm{H}), 7.19$ (ddd, $J=8.2,6.9,1.2$ Hz, 1H), 7.09 (ddd, $J=7.9,6.9,1.0 \mathrm{~Hz}, 1 \mathrm{H}), 6.87$ (q, $J=1.1 \mathrm{~Hz}, 1 \mathrm{H}), 4.05$ (t, $J=7.1$ $\mathrm{Hz}, 2 \mathrm{H}), 3.53-3.40(\mathrm{~m}, 2 \mathrm{H}), 3.27-3.20(\mathrm{~m}, 1 \mathrm{H}), 2.83(\mathrm{t}, J=9.6 \mathrm{~Hz}, 1 \mathrm{H}), 2.33(\mathrm{~d}, J$ $=1.1 \mathrm{~Hz}, 3 \mathrm{H}), 2.11-2.03(\mathrm{~m}, 1 \mathrm{H}), 1.99-1.92(\mathrm{~m}, 1 \mathrm{H}), 1.84-1.77(\mathrm{~m}, 2 \mathrm{H}), 1.487(\mathrm{~s}$, 9H), $1.33-1.25(\mathrm{~m}, 13 \mathrm{H}) \mathrm{ppm} .{ }^{13} \mathbf{C} \mathbf{N M R}\left(101 \mathrm{MHz}, \mathrm{CDCl}_{3}\right) \delta 154.8,136.4,128.8$, 125.5, 121.3, 119.1, 118.5, 110.1, 109.3, 79.0, 51.6, 46.2, 45.8, 38.9, 33.3, 31.7, 30.5, 29.8, 29.5, 29.4, 28.7, 28.3, 27.1, 9.7 ppm. IR (neat, $\mathrm{cm}^{-1}$ ): 2975, 2925, 2854, 1693, 1467, 1401, 1364, 1169, 1126, 1110, 882. HRMS calcd. for $\left(\mathrm{C}_{26} \mathrm{H}_{40} \mathrm{~N}_{2} \mathrm{NaO}_{2}\right)[\mathrm{M}+\mathrm{Na}]$ ${ }^{+}: 435.2982$ found 435.2990 .

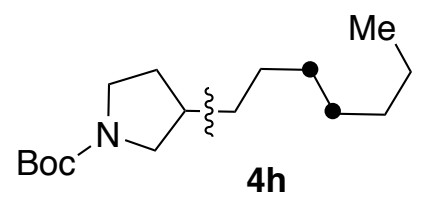

tert-butyl-3-heptylpyrrolidine-1-carboxylate (4h): Following the general procedure B, but using using 1-(1-(tert-butoxycarbonyl)pyrrolidin-3-yl)-2,4,6-triphenylpyridin-1ium tetrafluoroborate (1f, $112.8 \mathrm{mg}, 0.20 \mathrm{mmol})$ and trans-2-heptene (2t, $84 \mu \mathrm{L}, 0.60$ mmol), afforded $4 \mathbf{h}$ (43 $\mathrm{mg}, 80 \%$ yield) as a light yellow oil. In an independent experiment, $42 \mathrm{mg}$ (78\% yield) were obtained, giving an average of 79\% yield, 99:1 ratio. ${ }^{1} \mathbf{H}$ NMR $\left(400 \mathrm{MHz}, \mathrm{CDCl}_{3}\right) \delta 3.68-3.32(\mathrm{~m}, 2 \mathrm{H}), 3.30-3.11(\mathrm{~m}, 1 \mathrm{H}), 2.82(\mathrm{dt}$, $J=19.6,9.7 \mathrm{~Hz}, 1 \mathrm{H}), 2.12-2.01(\mathrm{~m}, 1 \mathrm{H}), 1.98-1.91(\mathrm{~m}, 1 \mathrm{H}), 1.44(\mathrm{~s}, 9 \mathrm{H}), 1.40-$ 
$1.11(\mathrm{~m}, 13 \mathrm{H}), 0.87(\mathrm{t}, J=6.8 \mathrm{~Hz}, 3 \mathrm{H}) \mathrm{ppm} .{ }^{13} \mathbf{C} \mathbf{N M R}\left(126 \mathrm{MHz}, \mathrm{CDCl}_{3}\right) \delta 154.8$, 79.0, 51.9, 51.4, 45.9, 45.5, 39.3, 38.4, 33.4, 32.1, 32.0, 31.3, 29.84, 29.80, 29.4, 28.7, 28.4, 22.8, 14.2 ppm. IR (neat, $\mathrm{cm}^{-1}$ ): 2977, 2959, 2924, 2855, 1696, 1455, 1399, 1364, 1254, 1168, 1135, 1111, 883. HRMS calcd. for $\left(\mathrm{C}_{16} \mathrm{H}_{31} \mathrm{NNaO}_{2}\right)[\mathrm{M}+\mathrm{Na}]^{+}: 292.2247$ found 292.2235 .

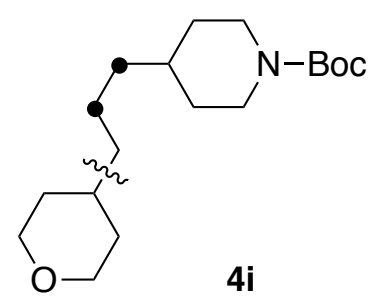

tert-butyl 4-(3-(tetrahydro-2H-pyran-4-yl)propyl)piperidine-1-carboxylate (4i):

Following the general procedure B, but using 2,4,6-triphenyl-1-(tetrahydro-2 $H$-pyran4-yl)pyridin-1-ium tetrafluoroborate (1e, $95.8 \mathrm{mg}, 0.20 \mathrm{mmol}$ ) and tert-butyl-4-(prop1-en-1-yl)piperidine-1-carboxylate (135,7 mg, $0.60 \mathrm{mmol}$ ), afforded $4 \mathbf{i}$ (42 mg, 68\% yield) as a colorless oil. In an independent experiment, $40 \mathrm{mg}$ (64\% yield) were obtained, giving an average of $66 \%$ yield, 99:1 ratio. ${ }^{1} \mathbf{H} \mathbf{N M R}\left(400 \mathrm{MHz}, \mathrm{CDCl}_{3}\right) \delta$ 4.04 (brs, 2H), 3.92 (ddt, $J=11.5,4.5,1.1 \mathrm{~Hz}, 2 \mathrm{H}), 3.34$ (td, $J=11.8,2.1 \mathrm{~Hz}, 2 \mathrm{H}$ ), $2.64(\mathrm{t}, J=12.7 \mathrm{~Hz}, 2 \mathrm{H}), 1.70-1.51(\mathrm{~m}, 4 \mathrm{H}), 1.46-1.38(\mathrm{~m}, 1 \mathrm{H}), 1.43(\mathrm{~s}, 9 \mathrm{H}), 1.35$ - $1.26(\mathrm{~m}, 3 \mathrm{H}), 1.25-1.15(\mathrm{~m}, 6 \mathrm{H}), 1.09-0.99(\mathrm{~m}, 2 \mathrm{H}) \mathrm{ppm} .{ }^{13} \mathrm{C}$ NMR (101 MHz, $\left.\mathrm{CDCl}_{3}\right) \delta 155.0,79.2,68.3,44.1,37.2,36.8,36.1,35.0,33.3,32.3,28.6,23.4$ ppm. IR (neat, $\mathrm{cm}^{-1}$ ): 2974, 2925, 2856, 2840, 1693, 1401, 1409, 1363, 1274, 1234, 1163, 1145, 1095, 975, 865. HRMS calcd. for $\left(\mathrm{C}_{18} \mathrm{H}_{33} \mathrm{NNaO}_{3}\right)[\mathrm{M}+\mathrm{Na}]^{+}: 334.2353$ found 334.2345 .

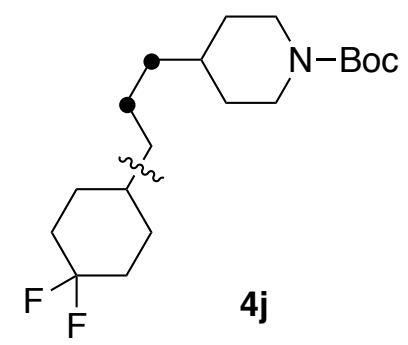

tert-butyl 4-(3-(4,4-difluorocyclohexyl)propyl)piperidine-1-carboxylate $\quad(4 \mathrm{j})$ :

Following the general procedure B, but using 1-(4,4-difluorocyclohexyl)-2,4,6triphenylpyridin-1-ium tetrafluoroborate (1c, $102.6 \mathrm{mg}, 0.20 \mathrm{mmol})$ and tert-butyl-4(prop-1-en-1-yl)piperidine-1-carboxylate (135,7 mg, $0.60 \mathrm{mmol}$ ), afforded 4j (42 mg, $61 \%$ yield) as a light yellow oil. In an independent experiment, $44 \mathrm{mg}$ (64\% yield) were obtained, giving an average of $62 \%$ yield, $22: 1$ regioisomeric ratio. ${ }^{1} \mathbf{H}$ NMR (400 MHz, 
$\left.\mathrm{CDCl}_{3}\right) \delta 4.04(\mathrm{brs}, 2 \mathrm{H}), 2.65(\mathrm{t}, J=12.8 \mathrm{~Hz}, 2 \mathrm{H}), 2.23-1.95(\mathrm{~m}, 2 \mathrm{H}), 1.77-1.70(\mathrm{~m}$, $3 \mathrm{H}), 1.67-1.58(\mathrm{~m}, 3 \mathrm{H}), 1.44(\mathrm{~s}, 9 \mathrm{H}), 1.39-1.16(\mathrm{~m}, 10 \mathrm{H}), 1.10-1.00(\mathrm{~m}, 2 \mathrm{H}) \mathrm{ppm}$. ${ }^{13}$ C NMR $\left(101 \mathrm{MHz}, \mathrm{CDCl}_{3}\right) \delta 155.0,124.0(\mathrm{dd}, J=241.8,239.4 \mathrm{~Hz}), 79.3,44.2,36.8$, 36.1, 35.9 (dd, $J=17.1,2.0 \mathrm{~Hz}), 33.7$ (dd, $J=25.4,22.2 \mathrm{~Hz}), 32.3,29.1,29.0,28.6$, 24.2 ppm. ${ }^{19}$ F NMR $\left(376 \mathrm{MHz}, \mathrm{CDCl}_{3}\right) \delta-91.51(\mathrm{~d}, J=234.3 \mathrm{~Hz}),-102.01(\mathrm{~d}, J=$ $234.0 \mathrm{~Hz}$ ). IR (neat, $\mathrm{cm}^{-1}$ ): 2975, 2927, 2851, 1689, 1448, 1420, 1364, 1274, 1243, $1166,1149,1117,1081,961,869$. HRMS calcd. for $\left(\mathrm{C}_{19} \mathrm{H}_{33} \mathrm{~F}_{2} \mathrm{NNaO}_{2}\right)[\mathrm{M}+\mathrm{Na}]^{+}$: 368.2372 found 368.2373 .

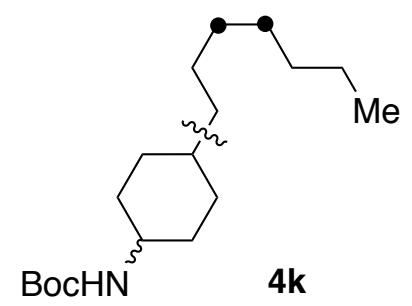

tert-butyl (4-heptylcyclohexyl)carbamate (4k): Following the general procedure B, but using using 1-(4-((tert-butoxycarbonyl)amino)cyclohexyl)-2,4,6-triphenylpyridin1-ium tetrafluoroborate $(\mathbf{1 d}, 118.4 \mathrm{mg}, 0.20 \mathrm{mmol})$ and trans-2-heptene $(84 \mu \mathrm{L}, 0.60$ mmol), afforded $4 \mathbf{k}$ (33 mg, 56\% yield) as a white solid. In an independent experiment, $35 \mathrm{mg}$ (59\% yield) were obtained, giving an average of 57\% yield, as a 1.5:1 mixture of diastereoisomers. Mp $69-70{ }^{\circ} \mathrm{C} .{ }^{1} \mathbf{H}$ NMR $\left(400 \mathrm{MHz}, \mathrm{CDCl}_{3}\right) \delta 4.64-4.3(\mathrm{~m}, 1 \mathrm{H})$, $3.72-3.33(\mathrm{~m}, 1 \mathrm{H}), 2.00-1.93(\mathrm{~m}, 1 \mathrm{H}), 1.76-1.71(\mathrm{~m}, 1 \mathrm{H}), 1.65-1.53(\mathrm{~m}, 2 \mathrm{H})$, $1.43(\mathrm{~d}, J=3.0 \mathrm{~Hz}, 9 \mathrm{H}), 1.35-0.91(\mathrm{~m}, 17 \mathrm{H}), 0.87(\mathrm{td}, J=6.9,1.9 \mathrm{~Hz}, 3 \mathrm{H}) \mathrm{ppm} .{ }^{13} \mathbf{C}$ NMR $\left(101 \mathrm{MHz}, \mathrm{CDCl}_{3}\right) \delta$ 155.4, 79.1, 50.2, 46.8, 37.02 (left), 37.02 (right), 35.8, $35.7,33.7,32.1,32.0,30.02,30.00,29.95,29.5,28.59,28.57,28.1,27.22,27.17,22.8$, 14.2 ppm. IR (neat, $\mathrm{cm}^{-1}$ ): 3370, 3726, 2920, 2849, 1683, 1515, 1453, 1388, 1363, 1174 , 1043, 783. HRMS calcd. for $\left(\mathrm{C}_{18} \mathrm{H}_{35} \mathrm{NNaO}_{2}\right)[\mathrm{M}+\mathrm{Na}]^{+}: 320.2560$ found 320.2558 . 


\section{Advanced Synthetic Intermediates (Scheme 3)}

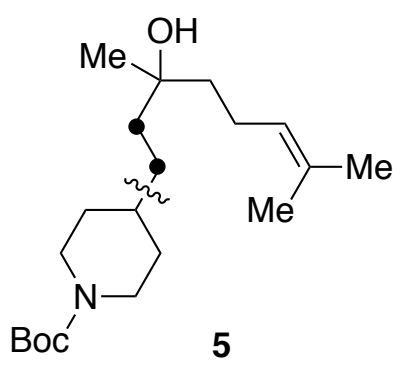

tert-butyl 4-(3-hydroxy-3,7-dimethyloct-6-en-1-yl)piperidine-1-carboxylate (5):

Following the general procedure, but using linalool $(106 \mu \mathrm{L}, 0.60 \mathrm{mmol})$, afforded 5 (27 mg, 40\% yield) as a colorless oil. In an independent experiment, $28 \mathrm{mg}$ (41\% yield) were obtained, giving an average of $40 \%$ yield. ${ }^{1} \mathbf{H}$ NMR $\left(400 \mathrm{MHz}, \mathrm{CDCl}_{3}\right) \delta 5.17-$ $5.09(\mathrm{~m}, 1 \mathrm{H}), 4.12-4.01(\mathrm{~m}, 2 \mathrm{H}), 2.72-2.59(\mathrm{~m}, 2 \mathrm{H}), 2.08-1.96(\mathrm{~m}, 2 \mathrm{H}), 1.70-$ $1.60(\mathrm{~m}, 8 \mathrm{H}), 1.50-1.45(\mathrm{~m}, 3 \mathrm{H}), 1.44(\mathrm{~s}, 9 \mathrm{H}), 1.34-1.22(\mathrm{~m}, 5 \mathrm{H}), 1.15(\mathrm{~s}, 3 \mathrm{H}), 1.13$ - $1.03(\mathrm{~m}, 2 \mathrm{H}) \mathrm{ppm} .{ }^{13} \mathbf{C} \mathbf{N M R}\left(101 \mathrm{MHz}, \mathrm{CDCl}_{3}\right) \delta 155.0,131.9,124.5,79.3,72.8$, 44.2, 41.6, 39.0, 36.7, 32.4, 30.8, 28.6, 27.0, 25.8, 22.8, 17.8 ppm. IR (neat, $\mathrm{cm}^{-1}$ ): 3451, 2969, 2927, 2855, 1672, 1424, 1365, 1277, 1244, 1157, 965, 866. HRMS calcd. for $\left(\mathrm{C}_{20} \mathrm{H}_{37} \mathrm{NNaO}_{3}\right)[\mathrm{M}+\mathrm{Na}]^{+}: 362.2666$ found 362.2654 .

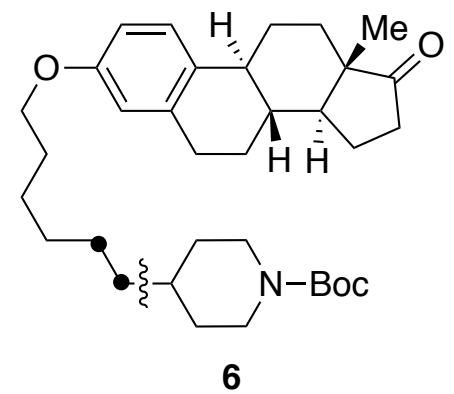

tert-butyl $\quad 4-(6-((8 R, 9 S, 13 S, 14 S)-13-m e t h y l-17-0 x 0-7,8,9,11,12,13,14,15,16,17-$ decahydro- $6 H$-cyclopenta $[a]$ phenanthren-3-yl)oxy)hexyl)piperidine-1

carboxylate (6): Following the general procedure, but using $(8 R, 9 S, 13 S, 14 S)$-3-(hex5-en-1-yloxy)-13-methyl-6,7,8,9,11,12,13,14,15,16-decahydro-17H cyclopenta $[a]$ phenanthrene-17-one (202.9 mg, $0.60 \mathrm{mmol})$, afforded 6 (81 $\mathrm{mg}, 75 \%$ yield) as a white solid. In an independent experiment, $81 \mathrm{mg}$ (75\% yield) were obtained, giving an average of $75 \%$ yield. Mp $119-119{ }^{\circ} \mathrm{C} .[\mathrm{a}]^{20} \mathrm{D}=+84.6^{\circ}\left(\mathrm{c}=0.1, \mathrm{CH}_{2} \mathrm{Cl}_{2}\right) .{ }^{1} \mathbf{H}$ NMR $(400$ $\left.\mathrm{MHz}, \mathrm{CDCl}_{3}\right) \delta 7.18(\mathrm{dd}, J=8.7,1.1 \mathrm{~Hz}, 1 \mathrm{H}), 6.70(\mathrm{dd}, J=8.6,2.8 \mathrm{~Hz}, 1 \mathrm{H}), 6.63(\mathrm{~d}$, $J=2.7 \mathrm{~Hz}, 1 \mathrm{H}), 4.06(\mathrm{~d}, J=13.2 \mathrm{~Hz}, 2 \mathrm{H}), 3.92(\mathrm{t}, J=6.5 \mathrm{~Hz}, 2 \mathrm{H}), 2.93-2.83(\mathrm{~m}, 2 \mathrm{H})$, 
$2.66(\mathrm{td}, J=12.8,2.7 \mathrm{~Hz}, 2 \mathrm{H}), 2.54-2.44(\mathrm{~m}, 1 \mathrm{H}), 2.41-2.35(\mathrm{~m}, 1 \mathrm{H}), 2.29-2.19$ $(\mathrm{m}, 1 \mathrm{H}), 2.18-1.90(\mathrm{~m}, 4 \mathrm{H}), 1.79-1.69(\mathrm{~m}, 2 \mathrm{H}), 1.67-1.52(\mathrm{~m}, 5 \mathrm{H}), 1.48-1.40(\mathrm{~m}$, $5 \mathrm{H}), 1.45(\mathrm{~s}, 9 \mathrm{H}), 1.36-1.30(\mathrm{~m}, 5 \mathrm{H}), 1.25-1.21(\mathrm{~m}, 2 \mathrm{H}), 1.11-1.01(\mathrm{~m}, 2 \mathrm{H}), 0.90$ (s, 3H) ppm. ${ }^{13} \mathbf{C}$ NMR (101 MHz, $\left.\mathrm{CDCl}_{3}\right) \delta 220.9,157.2,154.9,137.7,126.3,114.6$, 112.1, 79.1, 67.9, 50.4, 48.0, 44.1, 44.0, 38.4, 36.5, 36.0, 35.9, 32.2, 31.6, 29.7, 29.6, 29.3, 28.5, 26.58, 26.55, 26.1, 25.9, 21.6, 13.9 ppm. IR (neat, $\mathrm{cm}^{-1}$ ): 2926, 2851, 2837 , 1735, 1694, 1473, 1407, 1366, 1276, 1239, 1165, 1148, 1096, 1056, 1007, 869. HRMS calcd. for $\left(\mathrm{C}_{34} \mathrm{H}_{51} \mathrm{NNaO}_{4}\right)[\mathrm{M}+\mathrm{Na}]^{+}: 560.3710$ found 560.3720 .

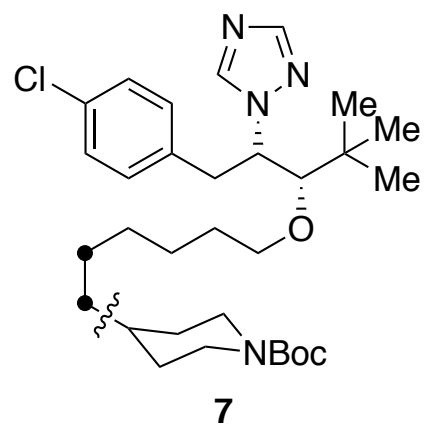

tert-butyl 4-(6-(((2S,3S)-1-(4-chlorophenyl)-4,4-dimethyl-2-(1H-1,2,4-triazol-1yl)pentan-3-yl)oxy)hexyl)piperidine-1-carboxylate (7): Following the general procedure, but using 1-(1-(tert-butoxycarbonyl)piperidin-4-yl)-2,4,6-triphenylpyridin1-ium tetrafluoroborate (1a, $231.2 \mathrm{mg}, 0.40 \mathrm{mmol}), 1-((2 \mathrm{R}, 3 \mathrm{R})-1-(4-c h l o r o p h e n y l)-3-$ (hex-5-en-1-yloxy)-4,4-dimethylpentan-2-yl)-1H-1,2,4-triazole (2ad, 75 mg, 0.20 $\mathrm{mmol}), \mathrm{NiBr}_{2} \cdot \mathrm{DME}$ (15 mol\%, $\left.9.3 \mathrm{mg}\right)$ and $\mathbf{L 4}(22.5 \mathrm{~mol} \%, 12 \mathrm{mg})$ afforded 7 (57 mg, $51 \%$ yield) as a light yellow oil. In an independent experiment, $57 \mathrm{mg}$ (51\% yield) were obtained, giving an average of $51 \%$ yield. ${ }^{1} \mathbf{H} \mathbf{~ N M R}\left(400 \mathrm{MHz}, \mathrm{CDCl}_{3}\right){ }^{1} \delta 8.39(\mathrm{~s}, 1 \mathrm{H})$, $7.74(\mathrm{~s}, 1 \mathrm{H}), 7.21-7.13(\mathrm{~m}, 2 \mathrm{H}), 6.95-6.87(\mathrm{~m}, 2 \mathrm{H}), 4.81-4.77(\mathrm{~m}, 1 \mathrm{H}), 4.05(\mathrm{~s}$, $2 \mathrm{H}), 3.66(\mathrm{t}, J=6.5 \mathrm{~Hz}, 2 \mathrm{H}), 3.23-3.05(\mathrm{~m}, 3 \mathrm{H}), 2.65(\mathrm{t}, J=12.7 \mathrm{~Hz}, 2 \mathrm{H}), 1.70-1.58$ $(\mathrm{m}, 4 \mathrm{H}), 1.43(\mathrm{~s}, 9 \mathrm{H}), 1.42-1.39(\mathrm{~m}, 1 \mathrm{H}), 1.36-1.28(\mathrm{~m}, 5 \mathrm{H}), 1.22(\mathrm{~d}, J=6.9 \mathrm{~Hz}$, $3 \mathrm{H}), 1.10-1.00(\mathrm{~m}, 2 \mathrm{H}), 0.72(\mathrm{~s}, 9 \mathrm{H}) \mathrm{ppm} .{ }^{13} \mathbf{C} \mathbf{N M R}\left(101 \mathrm{MHz}, \mathrm{CDCl}_{3}\right) \delta 155.0$, 149.8, 143.6, 135.4, 132.9, 130.0, 128.9, 87.2, 79.2, 75.6, 63.6, 44.2, 40.8, 36.6, 36.3, 36.1, 32.3, 30.7, 29.9, 28.6, 26.7, 26.54, 26.50 ppm. IR (neat, $\mathrm{cm}^{-1}$ ): 3076, 2982, 2934, 2865, 1457, 1380, 1254, 1210, 1168, 1111, 1068, 999, 916. HRMS calcd. for $\left(\mathrm{C}_{31} \mathrm{H}_{50} \mathrm{ClN}_{4} \mathrm{O}_{3}\right)[\mathrm{M}+\mathrm{H}]^{+}: 561.3566$ found 561.3578. 


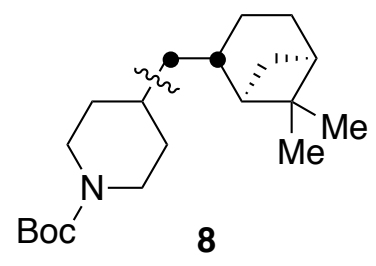

tert-butyl 4-(((1S,5S)-6,6-dimethylbicyclo[3.1.1]heptan-2-yl)methyl)piperidine-1carboxylate (8): Following the general procedure B, but using (-)- $\beta$-pinene $(93.6 \mu \mathrm{L}$, $0.60 \mathrm{mmol}$ ), afforded 8 (48 $\mathrm{mg}, 75 \%$ yield) as a colorless oil. In an independent experiment, $49 \mathrm{mg}$ (76\% yield) were obtained, giving an average of $75 \%$ yield, as a 97:3 mixture of diastereoisomers. $[\mathrm{a}]^{20} \mathrm{D}^{=}+4.0^{\circ}\left(\mathrm{c}=0.1, \mathrm{CH}_{2} \mathrm{Cl}_{2}\right){ }^{1} \mathbf{H} \mathbf{N M R}(400 \mathrm{MHz}$, $\left.\mathrm{CDCl}_{3}\right) \delta 5.06(\mathrm{dt}, J=2.5,1.3 \mathrm{~Hz}, 1 \mathrm{H}), 4.05$ (brs, 2H), 2.67 (q, $\left.J=14.2 \mathrm{~Hz}, 2 \mathrm{H}\right), 1.96$ - $1.77(\mathrm{~m}, 2 \mathrm{H}), 1.75-1.50(\mathrm{~m}, 6 \mathrm{H}), 1.44$ (s, 9H), 1.42 - 1.35 (m, 1H), $1.34-1.08$ (m, 4H), 0.97 (td, $J=10.2,5.1 \mathrm{~Hz}, 2 \mathrm{H}), 0.90$ (s, 3H), 0.75 (s, 3H) ppm. ${ }^{13} \mathrm{C}$ NMR (101 $\left.\mathrm{MHz}, \mathrm{CDCl}_{3}\right) \delta 155.0,133.6,131.1,79.2,44.2,40.2,37.0,34.7,34.02,33.97,31.6$, 30.5, 29.2, 28.6, 24.9, 23.6, 23.2 ppm. IR (neat, $\mathrm{cm}^{-1}$ ): 2959, 2923, 2862, 1692, 1421, 1364, 1278, 1245, 1220, 1165, 1150, 1125, 1069, 967. HRMS calcd. for $\left(\mathrm{C}_{20} \mathrm{H}_{35} \mathrm{NNaO}_{2}\right)[\mathrm{M}+\mathrm{Na}]^{+}: 344.2560$ found 344.2558 .
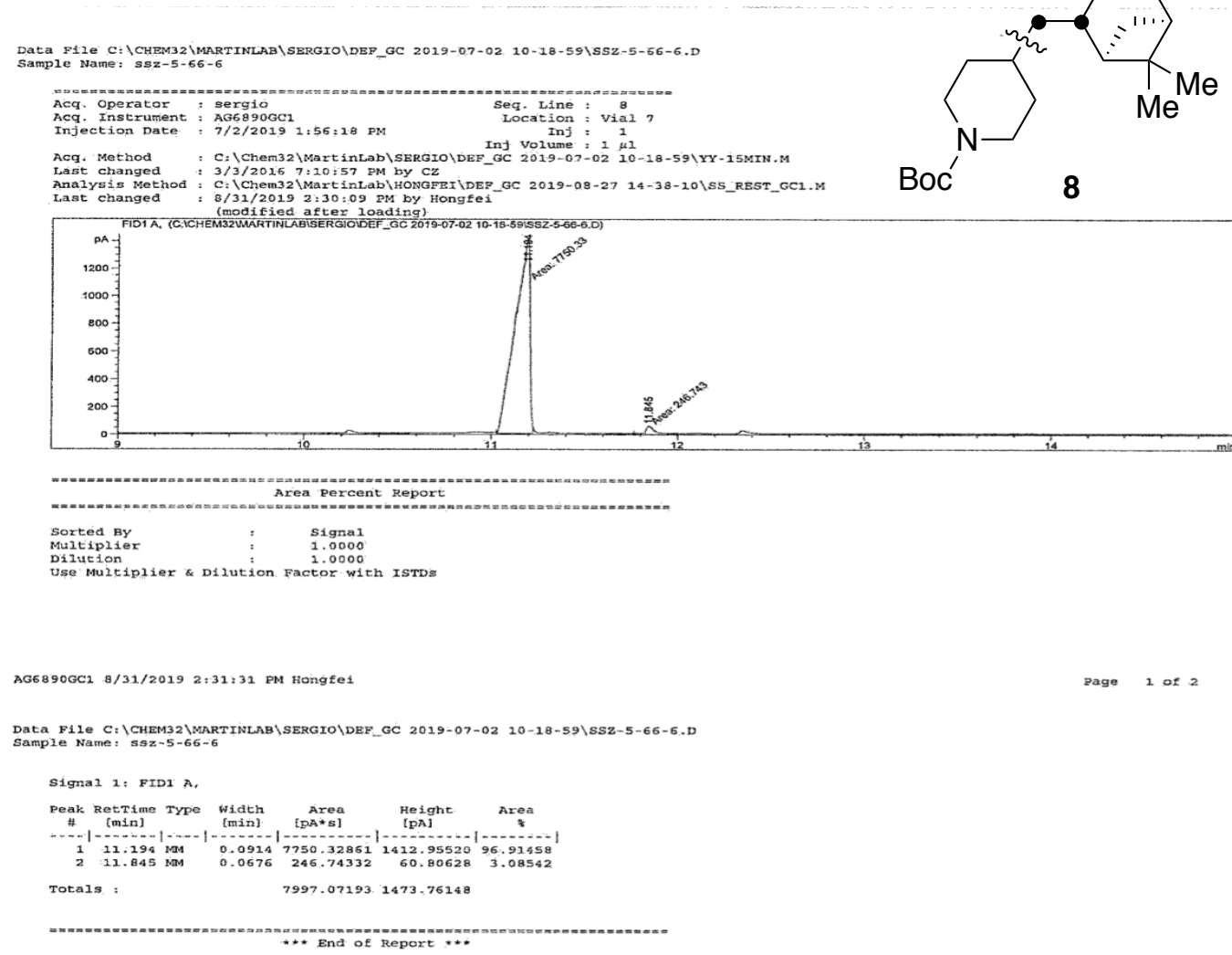


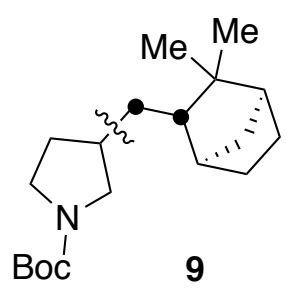

tert-butyl -(3R)-3-(((1S,4R)-3,3-dimethylbicyclo[2.2.1] heptan-2-yl)methyl)

pyrrolidine-1-carboxylate (9): Following the general procedure B, but using 1-(1(tert-butoxycarbonyl)pyrrolidin-3-yl)-2,4,6-triphenylpyridin-1-ium tetrafluoroborate ( 1f, $112.8 \mathrm{mg}, 0.20 \mathrm{mmol})$ and camphene $(96.9 \mu \mathrm{L}, 0.60 \mathrm{mmol})$, afforded $9(31 \mathrm{mg}, 50 \%$ yield) as a colorless oil. In an independent experiment, $25 \mathrm{mg}$ (41\% yield) were obtained, giving an average of 46\% yield, as a 46:40:7:7 mixture of diastereoisomers. ${ }^{1} \mathbf{H}$ NMR $\left(400 \mathrm{MHz}, \mathrm{CDCl}_{3}\right) \delta 3.61-3.35$ (m, 2H), $3.26-3.15(\mathrm{~m}, 1 \mathrm{H}), 2.94-2.71$ (m, 1H), $2.18-1.86(\mathrm{~m}, 3 \mathrm{H}), 1.74-1.53(\mathrm{~m}, 3 \mathrm{H}), 1.45(\mathrm{~s}, 9 \mathrm{H}), 1.44-1.10(\mathrm{~m}, 7 \mathrm{H})$, $1.07-0.74(\mathrm{~m}, 7 \mathrm{H}) \mathrm{ppm} .{ }^{13} \mathbf{C}$ NMR $\left(101 \mathrm{MHz}, \mathrm{CDCl}_{3}\right) \delta 154.8,79.0,52.9,52.5,52.1$, 51.6, 51.3, 49.5, 49.4, 49.2, 49.1, 48.9, 48.8, 48.6, 46.0, 45.9, 45.7, 45.6, 44.51, 44.48, $44.4,41.3$, 41.2, 41.1, 41.0, 40.6, 38.42, 38.40, 38.2, 38.11, 38.10, 37.79, 37.77, 37.6, $37.52,37.46,37.14,37.11,36.6,35.9,33.0,32.5,32.4,32.3,32.0,31.9,31.6,31.0,30.3$, $30.2,29.9,29.8,29.5,28.7,28.1,25.9,24.9,24.8,24.2,23.02,23.00,21.9,21.6,21.5$, 21.0, 20.2, 20.1 ppm. IR (neat, $\mathrm{cm}^{-1}$ ): 2933, 2874, 1695, 1478, 1455, 1399, 1364, 1255 , 1169, 1118, 883. HRMS calcd. for $\left(\mathrm{C}_{19} \mathrm{H}_{33} \mathrm{NNaO}_{2}\right)[\mathrm{M}+\mathrm{Na}]^{+}: 330.2404$ found 330.2404 . 

Data. File C: \CHEM32\MARTINLAB \LIANG \DEF_GC 2019-07-11 16-18-09\SSZ-5-125B.D
Sample Name: SSZ-5-125B

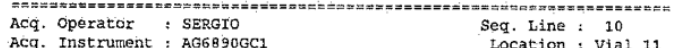

Acq. Instrument : AG68906C1 Location : vial 11

Inje volume : $1, \mu 1$

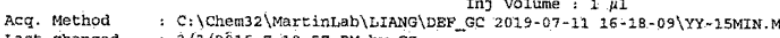

Last changed : 3/3/2016 7:10:57 PM by Cz

Analysis Method : C: \Chem32\MartinLab\HONGFEI \DEF_GC 2019-08-27 14-38-10\SS_REST_GC1.M

Last changed

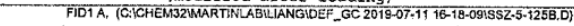
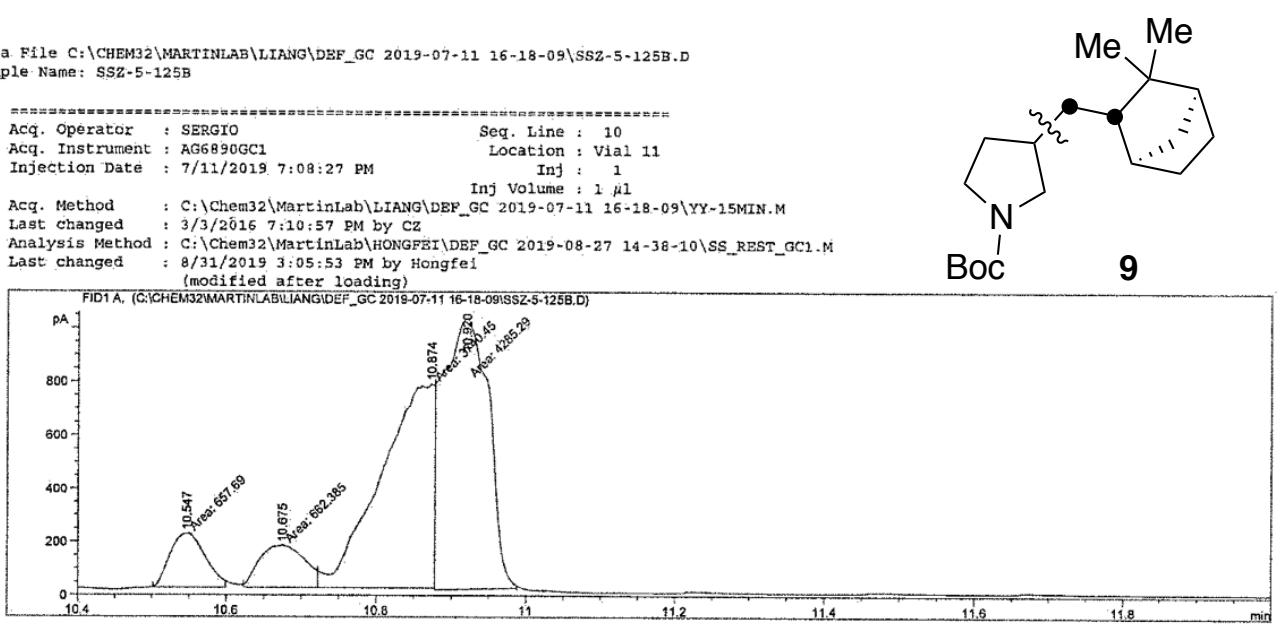

Area Percent Report

Sorted By $\quad: \quad$ Signal

$\begin{array}{lll}\text { Multiplier } & : & 1.0000 \\ \text { Dilution } & : & 1.0000\end{array}$

Use Multiplier \& Dilution Factor with ISTD

AG6890GC1. 8/31/2019 3:14:29 PM Hongfe

Data File C: \CHEM32 \MARTINLAB\LIANG\DEF_GC 2019-07-11 16-18-09\SSZ-5-125B.D

Signa1 1: FID1 A,

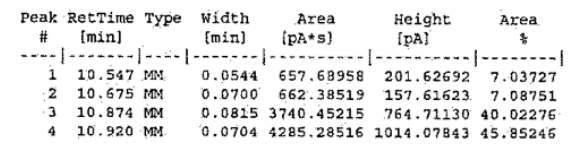

Totals : 9345.812072138 .03288

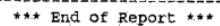

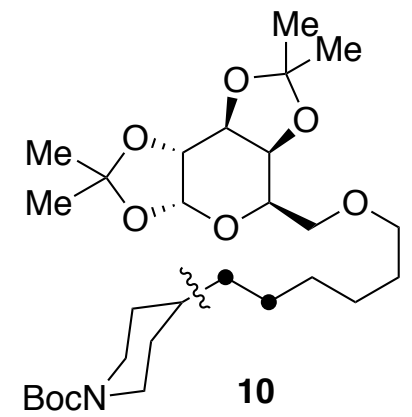

tert-butyl-4-(6-(((3a $R, 5 R, 5 \mathrm{a} S, 8 \mathrm{a} S, 8 \mathrm{~b} R)-2,2,7,7-t e t r a m e t h y l t e t r a h y d r o-5 H$-bis ([1,3]dioxolo)[4,5-b:4',5'-d]pyran-5-yl)methoxy)hexyl)piperidine-1-carboxylate

(10): Following the general procedure, but using ( $3 \mathrm{a} R, 5 R, 5 \mathrm{a} S, 8 \mathrm{a} S, 8 \mathrm{~b} R)-5-(($ hex-5-en1-yloxy)methyl)-2,2,7,7-tetramethyltetrahydro-5H-bis([1,3]dioxolo)[4,5-b:4',5'd]pyran (2ae, $195 \mathrm{mg}, 0.60 \mathrm{mmol}$ ), afforded 10 (61 mg, 58\% yield) as a colorless oil. In an independent experiment, $61 \mathrm{mg}$ (58\% yield) were obtained, giving an average of $58 \%$ yield. ${ }^{1} \mathbf{H}$ NMR $\left(400 \mathrm{MHz}, \mathrm{CDCl}_{3}\right) \delta 5.51(\mathrm{~d}, J=5.0 \mathrm{~Hz}, 1 \mathrm{H}), 4.57(\mathrm{dd}, J=7.9$, 
$2.4 \mathrm{~Hz}, 1 \mathrm{H}), 4.28(\mathrm{dd}, J=5.0,2.4 \mathrm{~Hz}, 1 \mathrm{H}), 4.23(\mathrm{dd}, J=7.9,1.9 \mathrm{~Hz}, 1 \mathrm{H}), 4.03(\mathrm{~d}, J=$ $13.3 \mathrm{~Hz}, 2 \mathrm{H}), 3.93(\mathrm{td}, J=6.2,1.9 \mathrm{~Hz}, 1 \mathrm{H}), 3.64-3.52(\mathrm{~m}, 2 \mathrm{H}), 3.50-3.39(\mathrm{~m}, 2 \mathrm{H})$, $2.63(\mathrm{td}, J=12.9,2.7 \mathrm{~Hz}, 2 \mathrm{H}), 1.64-1.52(\mathrm{~m}, 5 \mathrm{H}), 1.51(\mathrm{~s}, 3 \mathrm{H}), 1.43(\mathrm{~s}, 9 \mathrm{H}), 1.42(\mathrm{~s}$, $3 \mathrm{H}), 1.31(\mathrm{~s}, 3 \mathrm{H}), 1.30(\mathrm{~s}, 3 \mathrm{H}), 1.28-1.16(\mathrm{~m}, 8 \mathrm{H}), 1.08-0.97(\mathrm{~m}, 2 \mathrm{H}) \mathrm{ppm} .{ }^{13} \mathrm{C}$ NMR $\left(101 \mathrm{MHz}, \mathrm{CDCl}_{3}\right) \delta 155.0,109.3,108.6,96.5,79.2,71.6,71.3,70.8,70.7,69.4$, 66.8, 44.2, 36.6, 36.1, 32.3, 29.72, 29.67, 28.6, 26.7, 26.2, 26.13, 26.09, 25.1, 24.6 ppm. IR (neat, $\mathrm{cm}^{-1}$ ): 2980, 2928, 2855, 1740, 1691, 1422, 1367, 1244, 1211, 1166, 1111, 1069, 1001, 892. HRMS calcd. for $\left(\mathrm{C}_{28} \mathrm{H}_{49} \mathrm{NNaO}_{8}\right)[\mathrm{M}+\mathrm{Na}]^{+}: 550.3350$ found 550.3334 .

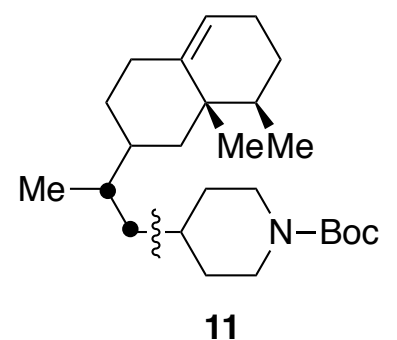

tert-butyl $\quad$ 4-(2-((8S,8aR)-8,8a-dimethyl-1,2,3,4,6,7,8,8a-octahydronaphthalen-2yl)propyl)piperidine-1-carboxylate (11): Following the general procedure B, but using valencene (133 $\mu \mathrm{L}, 0.60 \mathrm{mmol})$, afforded 11 (37 mg, 48\% yield) as a colorless oil. In an independent experiment, $34 \mathrm{mg}$ (44\% yield) were obtained, giving an average of $46 \%$ yield, as a 9:1 mixture of diastereoisomers. ${ }^{1} \mathbf{H}$ NMR $\left(400 \mathrm{MHz}, \mathrm{CDCl}_{3}\right) \delta 5.28$ (dt, $J=4.3,2.0 \mathrm{~Hz}, 1 \mathrm{H}), 4.05$ (brs, 2H), $2.91-2.57$ (m, 2H), $2.34-2.15$ (m, 1H), 2.12 - $1.80(\mathrm{~m}, 2 \mathrm{H}), 1.77-1.54(\mathrm{~m}, 5 \mathrm{H}), 1.44(\mathrm{~s}, 9 \mathrm{H}), 1.49-1.33(\mathrm{~m}, 5 \mathrm{H}), 1.29-1.17$ (m, $2 \mathrm{H}), 1.13-0.93(\mathrm{~m}, 4 \mathrm{H}), 0.94-0.74(\mathrm{~m}, 10 \mathrm{H}) \mathrm{ppm} .{ }^{13} \mathbf{C ~ N M R}\left(101 \mathrm{MHz}, \mathrm{CDCl}_{3}\right) \delta$ 155.0, 144.8, 144.8, 143.97 (left), 143.97 (right), 119.84, 119.80, 119.3, 119.2, 79.2, 44.39 , 44.35, 44.2, 43.9, 42.5, 41.9, 41.44, 41.42, 41.28, 41.27, 41.0, 40.8, 40.5, 38.8, 38.7, 38.2, 38.1, 38.0, 37.8, 35.1, 34.8, 34.3, 34.2, 34.0, 33.71, 33.69, 33.6, 33.5, 33.4, $33.1,32.9,32.71,32.68,31.9,31.7,31.28,31.26,30.0,29.8,28.6,28.1,28.0,27.33$, $27.30,26.0,18.68,18.67,18.20,18.18,16.49,16.45,16.2,16.1,15.9,15.8$ ppm. IR (neat, $\mathrm{cm}^{-1}$ ): 2965, 2918, 2853, 1693, 1422, 1365, 1277, 1246, 1170, 1149, 973, 910, 865. HRMS calcd. for $\left(\mathrm{C}_{25} \mathrm{H}_{43} \mathrm{NNaO}_{2}\right)[\mathrm{M}+\mathrm{Na}]^{+}: 412.3186$ found 412.3195 . 

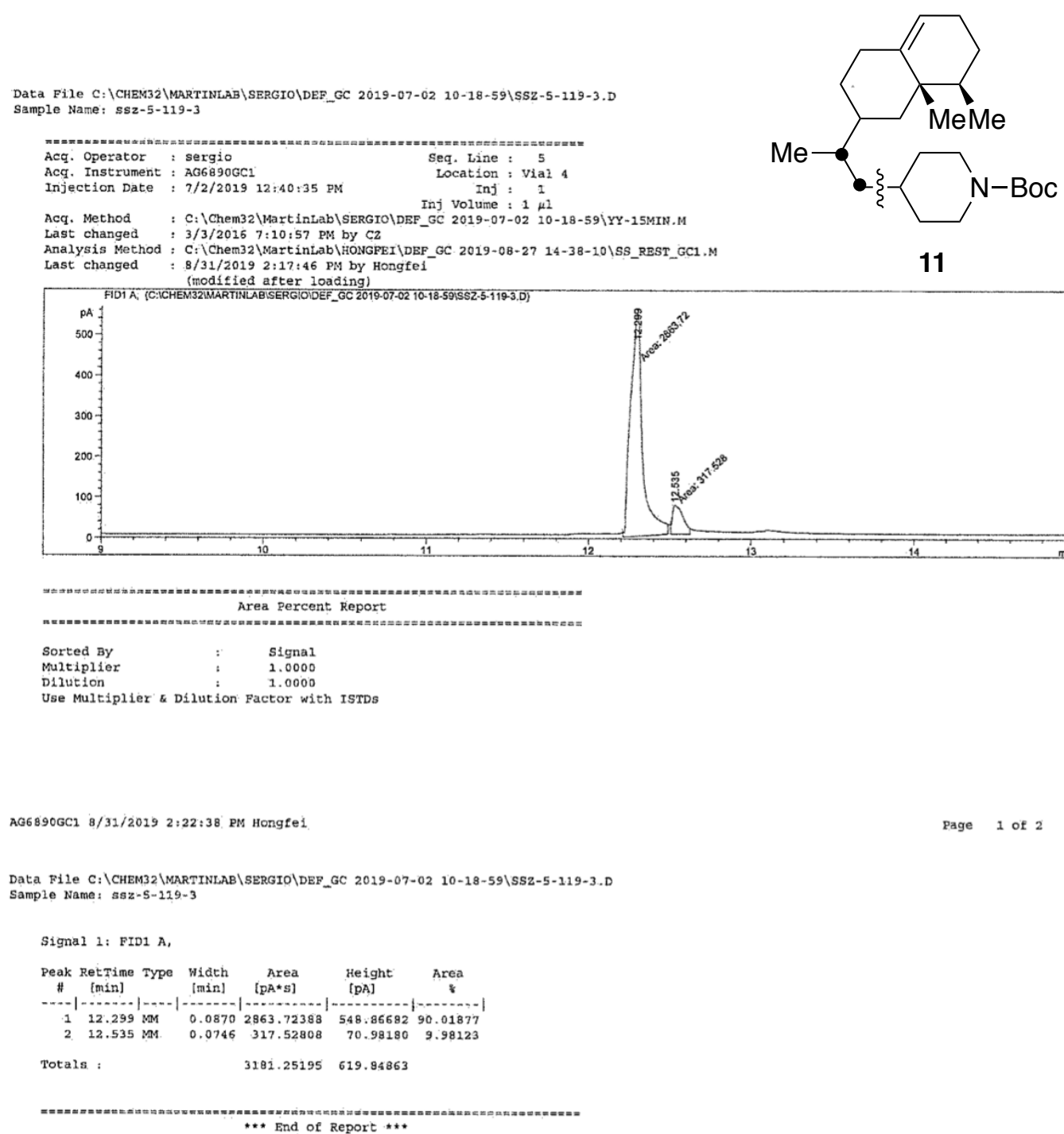

11

: 8/31/2019 2:17:46 PM by Hongfe 
1): 3027, 2933, 2858, 1734, 1496, 1453, 1368, 1263, 1193, 1158, 1030, 822. The observed spectral data are in agreement with the ones reported in literature. ${ }^{2}$

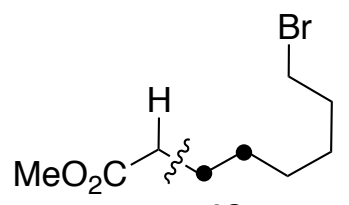

13

methyl 8-bromooctanoate (13): Following the general procedure, but using 1-(2methoxy-2-oxoethyl)-2,4,6-triphenylpyridin-1-ium tetrafluoroborate (1i, $93.6 \mathrm{mg}, 0.2$ mmol) and 6-bromo-1-hexene ( $80 \mu \mathrm{L}, 0.60 \mathrm{mmol})$ at $80{ }^{\circ} \mathrm{C}$, afforded $13(23 \mathrm{mg}, 49 \%$ yield) as a light yellow oil. In an independent experiment, $23 \mathrm{mg}$ (49\% yield) were obtained, giving an average of $49 \%$ yield. ${ }^{1} \mathbf{H}$ NMR $\left(400 \mathrm{MHz}, \mathrm{CDCl}_{3}\right) \delta 3.66(\mathrm{~s}, 3 \mathrm{H})$, $3.39(\mathrm{t}, J=6.8 \mathrm{~Hz}, 2 \mathrm{H}), 2.30(\mathrm{t}, J=7.5 \mathrm{~Hz}, 2 \mathrm{H}), 1.89-1.80(\mathrm{~m}, 2 \mathrm{H}), 1.66-1.58(\mathrm{~m}$, $2 \mathrm{H}), 1.45-1.39(\mathrm{~m}, 2 \mathrm{H}), 1.34-1.24(\mathrm{~m}, 4 \mathrm{H}) \mathrm{ppm} .{ }^{13} \mathbf{C} \mathbf{N M R}\left(101 \mathrm{MHz}, \mathrm{CDCl}_{3}\right) \delta$ 174.3, 51.6, 34.2, 34.0, 32.8, 29.1, 28.5, 28.1, 25.0 ppm. IR (neat, $\mathrm{cm}^{-1}$ ): 2931, 2856, $1736,1435,1361,1237,1194,1170,1122,1016,881$. The observed spectral data are in agreement with the ones reported in literature. ${ }^{3}$

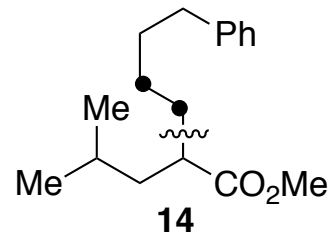

methyl-2-isobutyl-6-phenylhexanoate (14): Following the general procedure, but using 1-(1-methoxy-4-methyl-1-oxopentan-2-yl)-2,4,6-triphenylpyridin-1-ium tetrafluoroborate (1i, $104.6 \mathrm{mg}, 0.20 \mathrm{mmol})$ and 4-phenyl-1-butene (90 $\mu \mathrm{L}, 0.60 \mathrm{mmol})$, afforded 14 (26 mg, 50\% yield) as a light yellow oil. In an independent experiment, 26 mg (50\% yield) were obtained, giving an average of 50\% yield. ${ }^{1} \mathbf{H}$ NMR (400 MHz, $\left.\mathrm{CDCl}_{3}\right) \delta 7.30-7.24(\mathrm{~m}, 2 \mathrm{H}), 7.20-7.13(\mathrm{~m}, 3 \mathrm{H}), 3.65(\mathrm{~s}, 3 \mathrm{H}), 2.63-2.56(\mathrm{~m}, 2 \mathrm{H})$, $2.43(\mathrm{tt}, J=9.3,5.3 \mathrm{~Hz}, 1 \mathrm{H}), 1.65-1.42(\mathrm{~m}, 6 \mathrm{H}), 1.35-1.20(\mathrm{~m}, 3 \mathrm{H}), 0.88(\mathrm{t}, J=6.7$ $\mathrm{Hz}, 6 \mathrm{H}) \mathrm{ppm} .{ }^{13} \mathrm{C}$ NMR $\left(101 \mathrm{MHz}, \mathrm{CDCl}_{3}\right) \delta 177.3,142.7,128.5,128.4,125.8,51.5$, 43.8, 42.0, 35.9, 33.0, 31.5, 27.3, 26.4, 23.2, 22.3 ppm. IR (neat, $\mathrm{cm}^{-1}$ ): 3027, 2933,

\footnotetext{
2 Hanessian, S.; Moitessier, N.; Cantin, L.-D. Tetrahedron, 2001, 57, 6885.

${ }^{3}$ Perlikowska, W.; Mikołajczyk, M. Synthesis, 2009, 16, 2715.
} 
2858, 1734, 1496, 1453, 1368, 1263, 1193, 1155, 1030, 822. HRMS calcd. for $\left(\mathrm{C}_{17} \mathrm{H}_{26} \mathrm{NaO}_{2}\right)[\mathrm{M}+\mathrm{Na}]^{+}: 285.1825$ found 285.1822 .

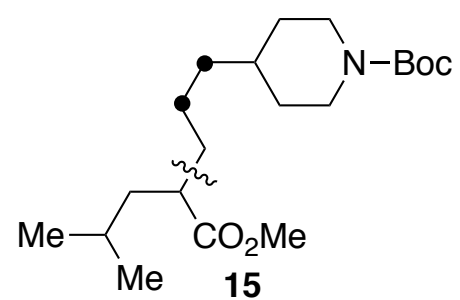

tert-butyl-4-(4-(methoxycarbonyl)-6-methylheptyl)piperidine-1-carboxylate (15):

Following the general procedure B, but using using 1-(1-methoxy-4-methyl-1oxopentan-2-yl)-2,4,6-triphenylpyridin-1-ium tetrafluoroborate (1j, $104.6 \mathrm{mg}, 0.20$ mmol) and tert-butyl-4-(prop-1-en-1-yl)piperidine-1-carboxylate (135,7 mg, 0.60 mmol), afforded 15 (35 mg, 49\% yield) as a light yellow oil. In an independent experiment, $38 \mathrm{mg}$ (53\% yield) were obtained, giving an average of 51\% yield, 26:1 ratio. ${ }^{1} \mathbf{H}$ NMR $\left(400 \mathrm{MHz}, \mathrm{CDCl}_{3}\right) \delta 4.04$ (brs, 2H), 3.64 (s, 3H), 2.63 (t, $J=12.4 \mathrm{~Hz}$, 2H), $2.41(\mathrm{tt}, J=9.3,5.2 \mathrm{~Hz}, 1 \mathrm{H}), 1.66-1.48(\mathrm{~m}, 5 \mathrm{H}), 1.43(\mathrm{~s}, 9 \mathrm{H}), 1.39-1.14(\mathrm{~m}$, $7 \mathrm{H}), 1.10-0.95(\mathrm{~m}, 2 \mathrm{H}), 0.86(\mathrm{dd}, J=7.8,6.5 \mathrm{~Hz}, 6 \mathrm{H}) \mathrm{ppm} .{ }^{13} \mathbf{C}$ NMR $(101 \mathrm{MHz}$, $\left.\mathrm{CDCl}_{3}\right) \delta 177.2,155.0,79.3,51.4,44.3,43.8,41.9,36.5,35.9,33.2,32.3,32.2,28.6$, 26.3, 24.6, 23.1, 22.2 ppm. IR (neat, $\mathrm{cm}^{-1}$ ): 2975, 2929, 2851, 1735, 1691, 1421, 1365, 1276, 1244, 1158, 972, 868. HRMS calcd. for $\left(\mathrm{C}_{20} \mathrm{H}_{37} \mathrm{NNaO}_{4}\right)[\mathrm{M}+\mathrm{Na}]^{+}: 378.2615$ found 378.2612 .

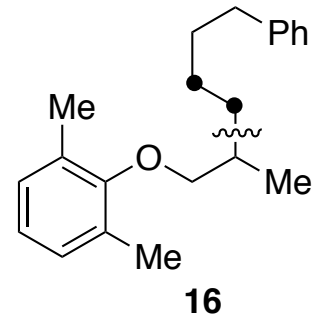

1,3-dimethyl-2-((2-methyl-6-phenylhexyl)oxy)benzene (16): Following the general procedure, but using 1-(1-(2,6-dimethylphenoxy)propan-2-yl)-2,4,6-triphenylpyridin1-ium tetrafluoroborate $(\mathbf{1 k}, 111.6 \mathrm{mg}, 0.2 \mathrm{mmol})$ and 4-phenyl-1-butene (90 $\mu \mathrm{L}, 0.60$ mmol), afforded 16 (38 mg, 64\% yield) as a light yellow oil. In an independent experiment, $35 \mathrm{mg}$ (59\% yield) were obtained, giving an average of $61 \%$ yield. ${ }^{1} \mathbf{H}$ NMR $\left(400 \mathrm{MHz}, \mathrm{CDCl}_{3}\right) \delta 7.34-7.26(\mathrm{~m}, 2 \mathrm{H}), 7.23-7.17(\mathrm{~m}, 3 \mathrm{H}), 7.02(\mathrm{dq}, J=7.2$, $0.7 \mathrm{~Hz}, 2 \mathrm{H}), 6.92(\mathrm{dd}, J=8.2,6.7 \mathrm{~Hz}, 1 \mathrm{H}), 3.66-3.48(\mathrm{~m}, 2 \mathrm{H}), 2.66(\mathrm{t}, J=7.7 \mathrm{~Hz}$, 2H), $2.01-1.93(\mathrm{~s}, 6 \mathrm{H}), 1.97(\mathrm{~m}, 1 \mathrm{H}), 1.75-1.59(\mathrm{~m}, 3 \mathrm{H}), 1.52-1.37(\mathrm{~m}, 2 \mathrm{H}), 1.34$ 
$-1.28(\mathrm{~m}, 1 \mathrm{H}), 1.10(\mathrm{~d}, J=6.7 \mathrm{~Hz}, 3 \mathrm{H}) \mathrm{ppm} .{ }^{13} \mathbf{C} \mathbf{N M R}\left(101 \mathrm{MHz}, \mathrm{CDCl}_{3}\right) \delta 156.0$, $142.8,131.0,128.8,128.4,128.3,125.6,123.6,77.1,36.0,34.3,33.4,31.8,26.8,17.2$, 16.3 ppm. IR (neat, $\mathrm{cm}^{-1}$ ): 3025, 2974, 2926, 2856, 1475, 1463, 1383, 1263, 1202, 1091, 1007, 980, 827. HRMS calcd. for $\left(\mathrm{C}_{21} \mathrm{H}_{29} \mathrm{O}\right)[\mathrm{M}+\mathrm{H}]^{+}: 297.2213$ found 297.2214.

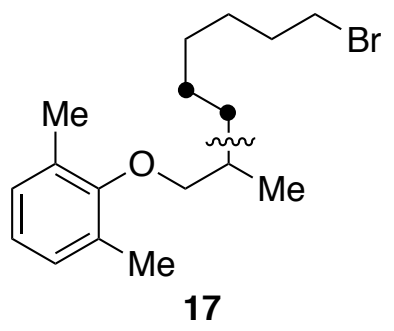

2-((8-bromo-2-methyloctyl)oxy)-1,3-dimethylbenzene (17): Following the general procedure, but using 1-(1-(2,6-dimethylphenoxy)propan-2-yl)-2,4,6-triphenylpyridin1-ium tetrafluoroborate $(\mathbf{1 k}, 111.6 \mathrm{mg}, 0.2 \mathrm{mmol})$ and 6-bromo1-hexene $(80 \mu \mathrm{L}, 0.60$ mmol), afforded 17 (37 mg, 57\% yield) as a light yellow oil. In an independent experiment, $35 \mathrm{mg}$ (54\% yield) were obtained, giving an average of $55 \%$ yield. ${ }^{1} \mathbf{H}$ NMR (400 MHz, $\left.\mathrm{CDCl}_{3}\right) \delta 7.01(\mathrm{~d}, J=7.2 \mathrm{~Hz}, 2 \mathrm{H}), 6.91(\mathrm{dd}, J=8.2,6.7 \mathrm{~Hz}, 1 \mathrm{H})$, $3.66-3.50(\mathrm{~m}, 2 \mathrm{H}), 3.42(\mathrm{t}, J=6.9 \mathrm{~Hz}, 2 \mathrm{H}), 2.28(\mathrm{~s}, 6 \mathrm{H}), 2.01-1.83(\mathrm{~m}, 3 \mathrm{H}), 1.64-$ $1.56(\mathrm{~m}, 1 \mathrm{H}), 1.49-1.43(\mathrm{~m}, 2 \mathrm{H}), 1.41-1.33(\mathrm{~m}, 3 \mathrm{H}), 1.31-1.22(\mathrm{~m}, 2 \mathrm{H}), 1.10(\mathrm{~d}, J$ $=6.7 \mathrm{~Hz}, 3 \mathrm{H}) \mathrm{ppm} .{ }^{13} \mathbf{C} \mathbf{N M R}\left(101 \mathrm{MHz}, \mathrm{CDCl}_{3}\right) \delta 156.0,131.1,128.9,123.7,77.3$, 34.4, 34.1, 33.5, 32.9, 29.2, 28.3, 27.1, 17.2, 16.4 ppm. IR (neat, $\mathrm{cm}^{-1}$ ): 2975, 2925, 2855, 1463, 1383, 1262, 1202, 1091, 1013, 981, 840. HRMS calcd. for $\left(\mathrm{C}_{17} \mathrm{H}_{28} \mathrm{BrO}_{2}\right)$ $[\mathrm{M}+\mathrm{H}]^{+}: 327.1318$ found 327.1320 .

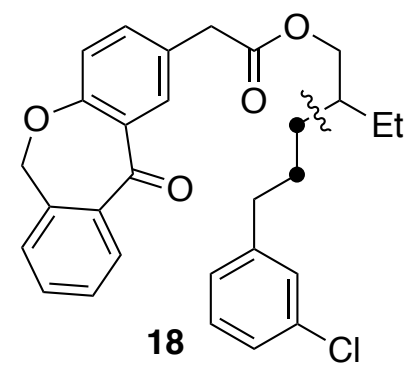

\section{5-(3-chlorophenyl)-2-ethylpentyl 2-(11-oxo-6,11-dihydrodibenzo[b,e]oxepin-2-}

yl)acetate (18): Following the general procedure, but using 1-(1-(2-(11-oxo-6,11dihydrodibenzo[b,e]oxepin-2-yl)acetoxy)butan-2-yl)-2,4,6-triphenylpyridin-1-ium tetrafluoroborate $(\mathbf{1 1}, 143 \mathrm{mg}, 0.20 \mathrm{mmol})$ and 1-allyl-3-chlorobenzene $(87.2 \mathrm{uL}, 0.60$ mmol), afforded 18 (68 mg, 71\% yield) as a colorless oil. In an independent experiment, $67 \mathrm{mg}$ (70\% yield) were obtained, giving an average of $70 \%$ yield. ${ }^{1} \mathbf{H}$ NMR $(400 \mathrm{MHz}$, $\left.\mathrm{CDCl}_{3}\right) \delta 8.13(\mathrm{~d}, J=2.4 \mathrm{~Hz}, 1 \mathrm{H}), 7.88(\mathrm{dd}, J=7.7,1.4 \mathrm{~Hz}, 1 \mathrm{H}), 7.55(\mathrm{td}, J=7.5,1.4$ 
$\mathrm{Hz}, 1 \mathrm{H}), 7.46(\mathrm{td}, J=7.6,1.3 \mathrm{~Hz}, 1 \mathrm{H}), 7.41(\mathrm{dd}, J=8.4,2.4 \mathrm{~Hz}, 1 \mathrm{H}), 7.36$ (dd, $J=7.4$, $1.3 \mathrm{~Hz}, 1 \mathrm{H}), 7.23-7.08(\mathrm{~m}, 3 \mathrm{H}), 7.04-7.00(\mathrm{~m}, 2 \mathrm{H}), 5.17$ (s, 2H), 4.02 (dd, $J=5.8$, $1.4 \mathrm{~Hz}, 2 \mathrm{H}), 3.63(\mathrm{~s}, 2 \mathrm{H}), 2.53(\mathrm{t}, J=7.7 \mathrm{~Hz}, 2 \mathrm{H}), 1.65-1.52(\mathrm{~m}, 3 \mathrm{H}), 1.37-1.25(\mathrm{~m}$, $4 \mathrm{H}), 0.85(\mathrm{t}, J=7.5 \mathrm{~Hz}, 3 \mathrm{H}) \mathrm{ppm} .{ }^{13} \mathbf{C} \mathbf{N M R}\left(101 \mathrm{MHz}, \mathrm{CDCl}_{3}\right) \delta 190.9,171.6,160.6$, $144.6,140.6,136.5,135.7,134.1,132.9,132.6,129.6,129.58,129.4,128.6,128.1$, $127.9,126.7,126.0,125.3,121.1,73.7,67.2,40.5,38.8,35.9,30.4,28.3,23.8,11.1$

ppm. IR (neat, $\mathrm{cm}^{-1}$ ): 3061, 2960, 2933, 2861, 1731, 1647, 1611, 1598, 1571, 1489, 1456, 1413, 1379, 1299, 1211, 1284, 1255, 1241, 1220, 1203, 1160, 1138, 1120, 1014. HRMS calcd. for $\left(\mathrm{C}_{29} \mathrm{H}_{29} \mathrm{ClNaO}_{4}\right)[\mathrm{M}+\mathrm{Na}]^{+}: 499.1647$ found 499.1644 .

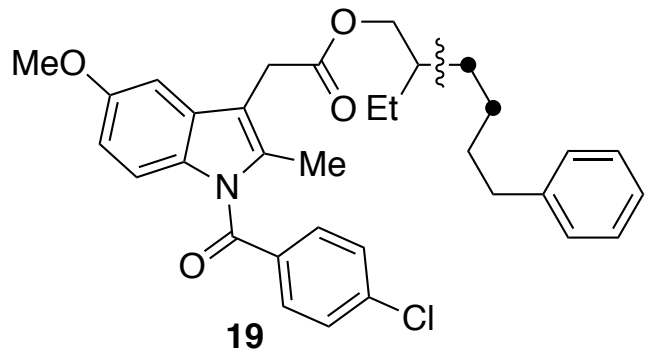

\section{2-ethyl-6-phenylhexyl 2-(1-(4-chlorobenzoyl)-5-methoxy-2-methyl-1H-indol-3-}

yl)acetate (19): Following the general procedure, but using 1-(1-(2-(1-(4chlorobenzoyl)-5-methoxy-2-methyl-1H-indol-3-yl)acetoxy)butan-2-yl)-2,4,6triphenylpyridin-1-ium tetrafluoroborate $(\mathbf{1 m}, 155 \mathrm{mg}, 0.20 \mathrm{mmol})$ and 4-phenyl-1butene (90 uL, $0.60 \mathrm{mmol})$, afforded 19 (65 $\mathrm{mg}, 60 \%$ yield) as a light yellow oil. In an independent experiment, $67 \mathrm{mg}$ (61\% yield) were obtained, giving an average of $60 \%$ yield. ${ }^{1} \mathbf{H}$ NMR $\left(400 \mathrm{MHz}, \mathrm{CDCl}_{3}\right) \delta 6.42-6.34(\mathrm{~m}, 2 \mathrm{H}), 6.23-6.15(\mathrm{~m}, 2 \mathrm{H}), 6.05-$ $5.96(\mathrm{~m}, 2 \mathrm{H}), 5.94-5.85(\mathrm{~m}, 3 \mathrm{H}), 5.71(\mathrm{~d}, J=2.5 \mathrm{~Hz}, 1 \mathrm{H}), 5.59(\mathrm{~d}, J=8.9 \mathrm{~Hz}, 1 \mathrm{H})$, $5.40(\mathrm{dd}, J=9.0,2.5 \mathrm{~Hz}, 1 \mathrm{H}), 2.75(\mathrm{~d}, J=5.7 \mathrm{~Hz}, 2 \mathrm{H}), 2.56$ (s, 3H), 2.40 (s, 2H), 1.32 $-1.23(\mathrm{~m}, 2 \mathrm{H}), 1.14(\mathrm{~s}, 3 \mathrm{H}), 0.33-0.21(\mathrm{~m}, 3 \mathrm{H}), 0.04--0.04(\mathrm{~m}, 6 \mathrm{H}),-0.42(\mathrm{t}, J=7.5$ $\mathrm{Hz}, 3 \mathrm{H}) \mathrm{ppm} .{ }^{13} \mathrm{C}$ NMR $\left(101 \mathrm{MHz}, \mathrm{CDCl}_{3}\right) \delta 171.1,168.4,156.2,142.7,139.4,136.0$, $134.1,131.3,130.9,130.8,129.2,128.5,128.4,125.8,115.1,112.9,111.8,101.5,67.4$, 55.8, 38.9, 35.9, 31.8, 30.7, 30.6, 26.5, 23.9, 13.4, 11.1 ppm. IR (neat, $\mathrm{cm}^{-1}$ ): 2958, 2929, 2857, 1731, 1682, 1603, 1477, 1455, 1355, 1313, 1260, 1222, 1164, 1141, 1066, 1036, 1015. HRMS calcd. for $\left(\mathrm{C}_{33} \mathrm{H}_{36} \mathrm{ClNNaO}_{4}\right)[\mathrm{M}+\mathrm{Na}]^{+}: 568.2225$ found 568.2227. 


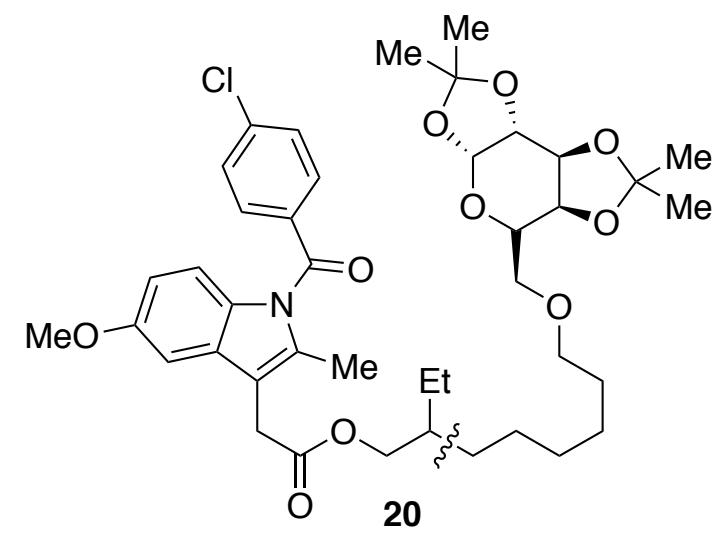

2-ethyl-8-(((3aR,5S,5aS,8aS,8bR)-2,2,7,7-tetramethyltetrahydro-5H-

bis([1,3]dioxolo)[4,5-b:4',5'-d] pyran-5-yl)methoxy)octyl 2-(1-(4-chlorobenzoyl)-5methoxy-2-methyl-1H-indol-3-yl)acetate (20): Following the general procedure, but using 1-(1-(2-(1-(4-chlorobenzoyl)-5-methoxy-2-methyl-1H-indol-3-yl)acetoxy) butan-2-yl)-2,4,6-triphenylpyridin-1-ium tetrafluoroborate (1 $\mathbf{m}, 155 \mathrm{mg}, 0.20 \mathrm{mmol})$ and $\quad(3 \mathrm{a} R, 5 R, 5 \mathrm{a} S, 8 \mathrm{a} S, 8 \mathrm{~b} R)-5$-((hex-5-en-1-yloxy)methyl)-2,2,7,7-tetramethyl tetrahydro-5H-bis([1,3]dioxolo)[4,5-b:4',5'-d]pyran (2ae, $195 \mathrm{mg}, 0.60 \mathrm{mmol}$ ), afforded 20 (87 mg, 57\% yield) as a light yellow oil. In an independent experiment, 83 $\mathrm{mg}\left(55 \%\right.$ yield) were obtained, giving an average of $56 \%$ yield. ${ }^{1} \mathbf{H} \mathbf{N M R}(400 \mathrm{MHz}$, $\left.\mathrm{CDCl}_{3}\right) \delta 7.70-7.62(\mathrm{~m}, 2 \mathrm{H}), 7.51-7.44(\mathrm{~m}, 2 \mathrm{H}), 6.96(\mathrm{~d}, J=2.5 \mathrm{~Hz}, 1 \mathrm{H}), 6.86(\mathrm{~d}, J$ $=9.0 \mathrm{~Hz}, 1 \mathrm{H}), 6.66(\mathrm{dd}, J=9.0,2.5 \mathrm{~Hz}, 1 \mathrm{H}), 5.53(\mathrm{~d}, J=5.0 \mathrm{~Hz}, 1 \mathrm{H}), 4.59(\mathrm{dd}, J=$ 7.9, $2.4 \mathrm{~Hz}, 1 \mathrm{H}), 4.30$ (dd, $J=5.0,2.4 \mathrm{~Hz}, 1 \mathrm{H}), 4.25$ (dd, $J=7.9,1.9 \mathrm{~Hz}, 1 \mathrm{H}), 4.00$ (dd, $J=5.8,2.6 \mathrm{~Hz}, 2 \mathrm{H}), 3.98-3.93(\mathrm{~m}, 1 \mathrm{H}), 3.83(\mathrm{~s}, 3 \mathrm{H}), 3.65(\mathrm{~s}, 2 \mathrm{H}), 3.63-3.53(\mathrm{~m}$, $2 \mathrm{H}), 3.50-3.40(\mathrm{~m}, 2 \mathrm{H}), 2.38(\mathrm{~s}, 3 \mathrm{H}), 1.57-1.50(\mathrm{~m}, 6 \mathrm{H}), 1.44(\mathrm{~s}, 3 \mathrm{H}), 1.33(\mathrm{~s}, 3 \mathrm{H})$, 1.32 (s, $3 \mathrm{H}), 1.30-1.24(\mathrm{~m}, 5 \mathrm{H}), 1.23-1.18(\mathrm{~m}, 5 \mathrm{H}), 0.82(\mathrm{t}, J=7.5 \mathrm{~Hz}, 3 \mathrm{H}) \mathrm{ppm}$. ${ }^{13}$ C NMR (101 MHz, $\left.\mathrm{CDCl}_{3}\right) \delta 171.1,168.4,156.2,139.4,135.9,134.1,131.3,130.9$, 130.8, 129.2, 115.1, 112.9, 111.8, 109.3, 108.6, 101.4, 96.5, 71.7, 71.4, 70.8, 70.8, 69.5, 67.4, 66.9, 55.8, 38.9, 30.8, 30.6, 29.9, 29.8, 29.7, 26.8, 26.2, 26.1, 25.1, 24.6, 23.9, 13.4, 11.1 ppm. IR (neat, $\mathrm{cm}^{-1}$ ): 2978, 2930, 2856, 1732, 1684, 1593, 1478, 1457, 1371, $1357,1314,1255,1212,1167,1110,1067,1000$. HRMS calcd. for $\left(\mathrm{C}_{41} \mathrm{H}_{54} \mathrm{ClNNaO}_{10}\right)$ $[\mathrm{M}+\mathrm{Na}]^{+}: 778.3328$ found 778.3358 . 


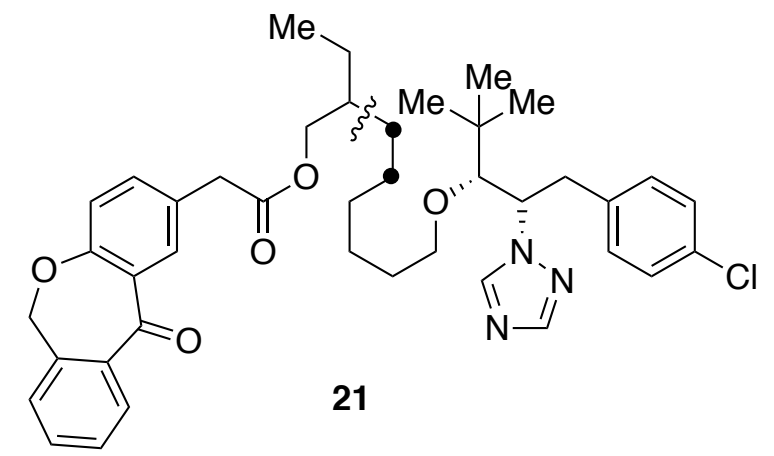

8-(((2S,3R)-1-(4-chlorophenyl)-4,4-dimethyl-2-(1H-1,2,4-triazol-1-yl)pentan-3yl)oxy)-2-ethyloctyl 2-(11-oxo-6,11-dihydrodibenzo[b,e]oxepin-2-yl)acetate (21):

Following the general procedure, but using 1-(1-(2-(11-oxo-6,11dihydrodibenzo[b,e]oxepin-2-yl)acetoxy)butan-2-yl)-2,4,6-triphenylpyridin-1-ium tetrafluoroborate (1l, $286 \mathrm{mg}, 0.40 \mathrm{mmol}), 1-((2 \mathrm{R}, 3 \mathrm{R})-1-(4-c h l o r o p h e n y l)-3-(h e x-5-$ en-1-yloxy)-4,4-dimethyl pentan -2-yl)-1H-1,2,4-triazole (2ad, 75 mg, $0.20 \mathrm{mmol}$ ), $\mathrm{NiBr}_{2} \cdot \mathrm{DME}$ (15 mol\%, $9.3 \mathrm{mg}$ ) and L4 (22.5 mol\%, $12 \mathrm{mg}$ ), afforded 21 (42 mg, 30\% yield) as a colorless oil. In an independent experiment, $43 \mathrm{mg}$ (30\% yield) were obtained, giving an average of $30 \%$ yield. ${ }^{1} \mathbf{H}$ NMR $\left(400 \mathrm{MHz}, \mathrm{CDCl}_{3}\right) \delta 8.44(\mathrm{~s}, 1 \mathrm{H})$, $8.12(\mathrm{~d}, J=2.4 \mathrm{~Hz}, 1 \mathrm{H}), 7.88(\mathrm{dd}, J=7.7,1.4 \mathrm{~Hz}, 1 \mathrm{H}), 7.78(\mathrm{~s}, 1 \mathrm{H}), 7.55(\mathrm{td}, J=7.4$, $1.4 \mathrm{~Hz}, 1 \mathrm{H}), 7.49-7.40(\mathrm{~m}, 2 \mathrm{H}), 7.35(\mathrm{dd}, J=7.4,1.3 \mathrm{~Hz}, 1 \mathrm{H}), 7.21-7.15(\mathrm{~m}, 2 \mathrm{H})$, $7.02(\mathrm{~d}, J=8.4 \mathrm{~Hz}, 1 \mathrm{H}), 6.92$ (d, $J=8.4 \mathrm{~Hz}, 2 \mathrm{H}), 5.18(\mathrm{~s}, 2 \mathrm{H}), 4.83-4.78(\mathrm{~m}, 1 \mathrm{H})$, $4.02(\mathrm{dd}, J=5.8,1.5 \mathrm{~Hz}, 2 \mathrm{H}), 3.72-3.60(\mathrm{~m}, 4 \mathrm{H}), 3.24-3.07(\mathrm{~m}, 3 \mathrm{H}), 1.68-1.55$ $(\mathrm{m}, 3 \mathrm{H}), 1.41-1.25(\mathrm{~m}, 10 \mathrm{H}), 0.86(\mathrm{t}, J=7.5 \mathrm{~Hz}, 3 \mathrm{H}), 0.74(\mathrm{~s}, 9 \mathrm{H}) \mathrm{ppm} .{ }^{13} \mathbf{C} \mathbf{N M R}$ $\left(101 \mathrm{MHz}, \mathrm{CDCl}_{3}\right) \delta 190.9,171.7,160.6,149.5,143.5,140.6,136.5,135.7,135.4$, 133.0, 132.9, 132.6, 130.0, 129.6, 129.4, 129.0, 128.2, 127.9, 125.3, 121.1, 87.3, 75.7, $73.8,67.4,63.8,40.9,40.5,38.9,36.4,30.8,30.7,30.0,26.8,26.6,26.5,23.9,11.2$ ppm. IR (neat, $\mathrm{cm}^{-1}$ ): 2956, 2928, 2858, 1731, 1648, 1612, 1599, 1490, 1456, 1412 , 1377, 1299, 1275, 1220, 1201, 1161, 1136, 1120, 1094, 1014. HRMS calcd. for $\left(\mathrm{C}_{41} \mathrm{H}_{50} \mathrm{ClN}_{3} \mathrm{NaO}_{5}\right)[\mathrm{M}+\mathrm{Na}]^{+}: 722.3331$ found 722.3354 . 


\section{Large Scale Reaction and Synthetic Applications}
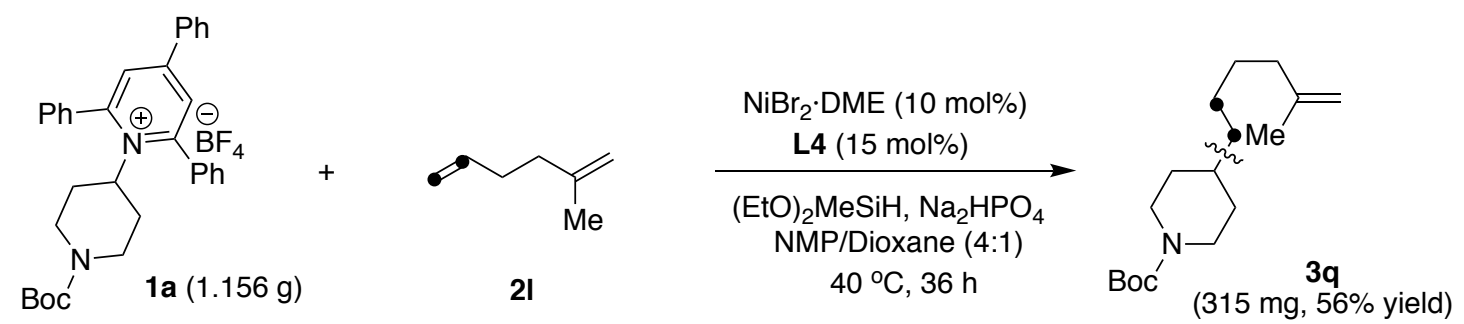

An oven-dried $25 \mathrm{~mL}$ schlenk tube containing a stirring bar was charged with $\mathrm{NiBr}_{2} \cdot \mathrm{DME}$ (62 mg, 10 mol\%), 4,4'-di-tert-butyl-2,2'-dipyridyl (L4, $80 \mathrm{mg}, 15 \mathrm{~mol} \%$ ), $\mathrm{Na}_{2} \mathrm{HPO}_{4}(568 \mathrm{mg}, 4.0 \mathrm{mmol})$ and 1-(1-(tert-butoxycarbonyl)piperidin-4-yl)-2,4,6triphenylpyridin-1-ium tetrafluoroborate (1a, $1.156 \mathrm{~g}, 2.0 \mathrm{mmol})$. Subsequently, the tube was sealed with a Teflon screw cap, then evacuated and back-filled with $\operatorname{Ar}(3$ times). Afterwards, 2-methylhexa-1,5-diene (674 $\mu \mathrm{L}, 5.0 \mathrm{mmol}),(\mathrm{EtO}){ }_{2} \mathrm{MeSiH}(960$ ul, $6 \mathrm{mmol}, 3.0$ equiv), NMP and 1,4-dioxane (4:1, $10 \mathrm{~mL})$ were added via syringe. Then, the tube was stirred at $40{ }^{\circ} \mathrm{C}$ for $36 \mathrm{~h}$. After the reaction was completed, and cool down to room temperature. The mixture was diluted with EtOAc, and washed with water. The aqueous phase was extracted with EtOAc $(3 \times 10 \mathrm{~mL})$, and the combined organic phases were dried over $\mathrm{MgSO}_{4}$ then concentrated. The residue was purified by column chromatography on silica gel (step gradient, Toluene to Hexane/EtOAc $=30: 1$ ), obtaining 3q as colourless oil $315 \mathrm{mg}$ (56\% yield).

\section{One-Pot Deaminative Alkylation without Isolation of Pyridinium Salts}
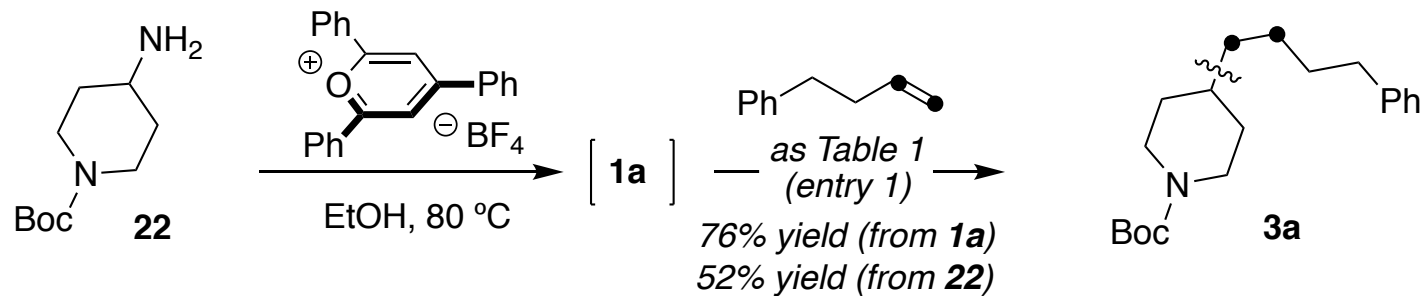

An oven-dried $50 \mathrm{~mL}$ round-bottomed flask containing a stirring bar was charged with tert-butyl 4-aminopiperidine-1-carboxylate (22, $200 \mathrm{mg}, 1.0 \mathrm{mmol})$ and 2,4,6triphenylpyrylium tetrafluoroborate $(400 \mathrm{mg}, 1.0 \mathrm{mmol})$. After then EtOH $(1.0 \mathrm{~mL})$ was added via syringe. The mixture was stirred and heated at reflux in an oil bath at $80-85^{\circ} \mathrm{C}$ for $4 \mathrm{~h}$. After the reaction was completed, cool down to room temperature and concentrated. To the flask was added $\mathrm{NiBr}_{2} \cdot \mathrm{DME}(31 \mathrm{mg}, 10 \mathrm{~mol} \%$ ), 4,4'-di-tert- 
butyl-2,2'-dipyridyl (L4, $40 \mathrm{mg}, 15 \mathrm{~mol} \%$ ), $\mathrm{Na}_{2} \mathrm{HPO}_{4}$ (284 mg, $2.0 \mathrm{mmol}$ ). Subsequently, the flask was sealed with a cap, then evacuated and back-filled with Ar (3 times). Afterwards, 4-phenyl-1-butene ( $450 \mu \mathrm{L}, 3.0 \mathrm{mmol}, 3.0$ equiv), (EtO) ${ }_{2} \mathrm{MeSiH}$ (480 $\mu \mathrm{L}, 3.0 \mathrm{mmol}, 3.0$ equiv), NMP and 1.4-dioxane (4:1, $5.0 \mathrm{~mL})$ were added via syringe. Then, the flask was stirred at $40^{\circ} \mathrm{C}$ for $24 \mathrm{~h}$. After the reaction was completed, and cool down to room temperature. The mixture was diluted with EtOAc, and washed with water. The aqueous phase was extracted with EtOAc $(3 \times 10 \mathrm{~mL})$, and the combined organic phases were dried over $\mathrm{MgSO}_{4}$ then concentrated. The residue was purified by column chromatography on silica gel (step gradient, Toluene to Hexane/EtOAc $=30: 1)$, obtaining 3a as light yellow oil $166 \mathrm{mg}(52 \%$ yield $)$.

\section{Regioconvergent Alkylation of Unrefined Mixtures of Olefins}
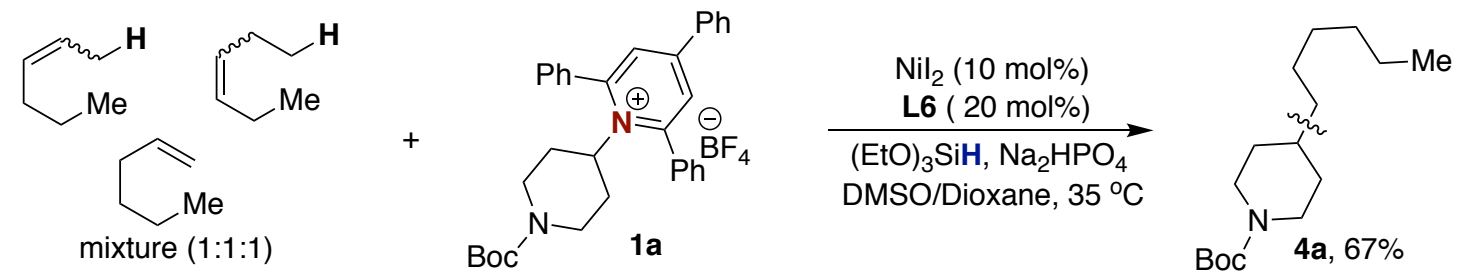

An oven-dried $8 \mathrm{~mL}$ screw-cap test tube containing a stirring bar was charged with $\mathrm{NiI}_{2}$ (6.2 mg, $10 \mathrm{~mol} \%$ ), 2-(6-methylpyridin-2-yl)-4,5-dihydrooxazole (L6, $6.4 \mathrm{mg}, 20$ mol\%), $\mathrm{Na}_{2} \mathrm{HPO}_{4}(113.6 \mathrm{mg}, 0.80 \mathrm{mmol})$ and 1-(1-(tert-butoxycarbonyl)piperidin-4yl)-2,4,6-triphenylpyridin-1-ium tetrafluoroborate (1a, $115.6 \mathrm{mg}, 0.20 \mathrm{mmol}$ ). Subsequently, the tube was sealed with a Teflon-lined screw cap, then evacuated and back-filled with $\operatorname{Ar}$ (3 times). Afterwards, the mixture of hexene (1:1:1, $74.6 \mu \mathrm{L}, 0.60$ mmol, 3.0 equiv), (EtO) ${ }_{3} \mathrm{SiH}(74 \mu \mathrm{L}, 0.40 \mathrm{mmol}, 2.0$ equiv), DMSO and 1.4-dioxane $(3: 1,1.2 \mathrm{~mL})$ were added via syringe. Then, the tube was stirred at $35^{\circ} \mathrm{C}$ for $24 \mathrm{~h}$. After the reaction was completed, the mixture was diluted with EtOAc, filtered through silica gel and concentrated under vacuum. The corresponding product was purified by column chromatography on silica gel (step gradient, Toluene to Hexane/EtOAc = 30:1), obtaining $4 \mathbf{a}$ as colorless oil $36 \mathrm{mg}$ (67\% yield), 99:1 ratio. 


\section{Utilization of Ethylene as Coupling Partner}<smiles></smiles>

$$
\begin{aligned}
& \underset{\mathrm{NiBr}_{2} \cdot \mathrm{DME}(10 \mathrm{~mol} \%)}{\mathrm{L4}(15 \mathrm{~mol} \%)} \\
& \underset{(\mathrm{EtO})_{2} \mathrm{MeSiH}, \mathrm{Na}_{2} \mathrm{HPO}_{4}}{\mathrm{NMP} / \text { Dioxane, } 40^{\circ} \mathrm{C}}
\end{aligned}
$$

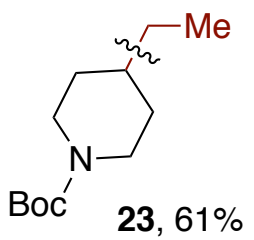

An oven-dried $8 \mathrm{~mL}$ screw-cap test tube containing a stirring bar was charged with $\mathrm{NiBr}_{2}$ ·DME (6.2 mg, 10 mol\%), 4,4'-di-tert-butyl-2,2'-dipyridyl (L4, $8.0 \mathrm{mg}, 15$ mol\%), $\mathrm{Na}_{2} \mathrm{HPO}_{4}(56.8 \mathrm{mg}, 0.40 \mathrm{mmol})$ and 1-(1-(tert-butoxycarbonyl)piperidin-4-yl)2,4,6-triphenylpyridin-1-ium tetrafluoroborate (1a, $115.6 \mathrm{mg}, \quad 0.20 \mathrm{mmol}$ ). Subsequently, the tube was sealed with a Teflon-lined screw cap, then evacuated and back-filled with ethylene (3 times). Afterwards, (EtO) ${ }_{2} \mathrm{MeSiH}(96 \mu \mathrm{L}, 0.60 \mathrm{mmol})$, NMP and 1.4-dioxane $(4: 1,1.0 \mathrm{~mL})$ were added via syringe. Then, the tube was stirred at $40{ }^{\circ} \mathrm{C}$ for $24 \mathrm{~h}$. The reaction under ethylene atmosphere with an ethylene balloon. After the reaction was completed, the mixture was diluted with EtOAc, filtered through silica gel and concentrated under vacuum. The corresponding product was purified by column chromatography on silica gel (step gradient, Hexane/EtOAc $=100: 1$ to 50:1 to 30:1), obtaining 23 as colorless oil $26 \mathrm{mg}$ (61\% yield). ${ }^{1} \mathbf{H}$ NMR (400 MHz, $\left.\mathrm{CDCl}_{3}\right) \delta$ 4.06 (brs, 2H), 2.65 (t, $J=12.7 \mathrm{~Hz}, 2 \mathrm{H}), 1.64$ (d, $J=13.0 \mathrm{~Hz}, 2 \mathrm{H}), 1.44$ (s, 9H), 1.30 $1.22(\mathrm{~m}, 3 \mathrm{H}), 1.05(\mathrm{qd}, J=11.9,11.0,6.3 \mathrm{~Hz}, 2 \mathrm{H}), 0.88(\mathrm{t}, J=7.2 \mathrm{~Hz}, 3 \mathrm{H}) \mathrm{ppm} .{ }^{13} \mathbf{C}$ NMR $\left(75 \mathrm{MHz}, \mathrm{CDCl}_{3}\right) \delta 154.9,79.1,44.1,37.7,31.8,29.2,28.5,11.1$ ppm. IR (neat, $\left.\mathrm{cm}^{-1}\right): 2965,2926,2852,1692,1418,1365,1229,1174,1149,1079$. The observed spectral data are in agreement with the ones reported in literature. ${ }^{4}$

\footnotetext{
${ }^{4}$ Dai, X.-J.; Li, C.-J. J. Am. Chem. Soc. 2016, 138, 5433
} 


\section{Mechanistic Experiments}

A priori, one might argue at first sight that two conceivable pathways might be viable for our catalytic deaminative alkylation of unactivated olefins: (a) single-electron transfer (SET) from low-valent $\mathrm{Ni}(0) \mathrm{L}_{\mathrm{n}}$ complexes to $\mathbf{1}$ followed by addition of the resulting alkyl radical to the olefin, or (b) initial hydrometallation of a nickel hydride to an olefin and subsequent addition to $\mathbf{1}$. At present, we believe that the former manifold makes a minor contribution, if any, to productive $s p^{3}-s p^{3}$ bond-formation due to an unfavorable polaritymismatch addition of nucleophilic radical intermediates to electron-rich olefins. This notion is further supported by the outcome observed for $\mathbf{2 5}$ (see Figure below). If an initial SET to $\mathbf{1}$ is operative, radical addition to the olefin would ultimately lead to C3functionalized product 26. It is worth noting, however, that these experiments do not allow us to rule out other mechanistic pathways, such as the intermediacy of $N$-stabilized radicals.

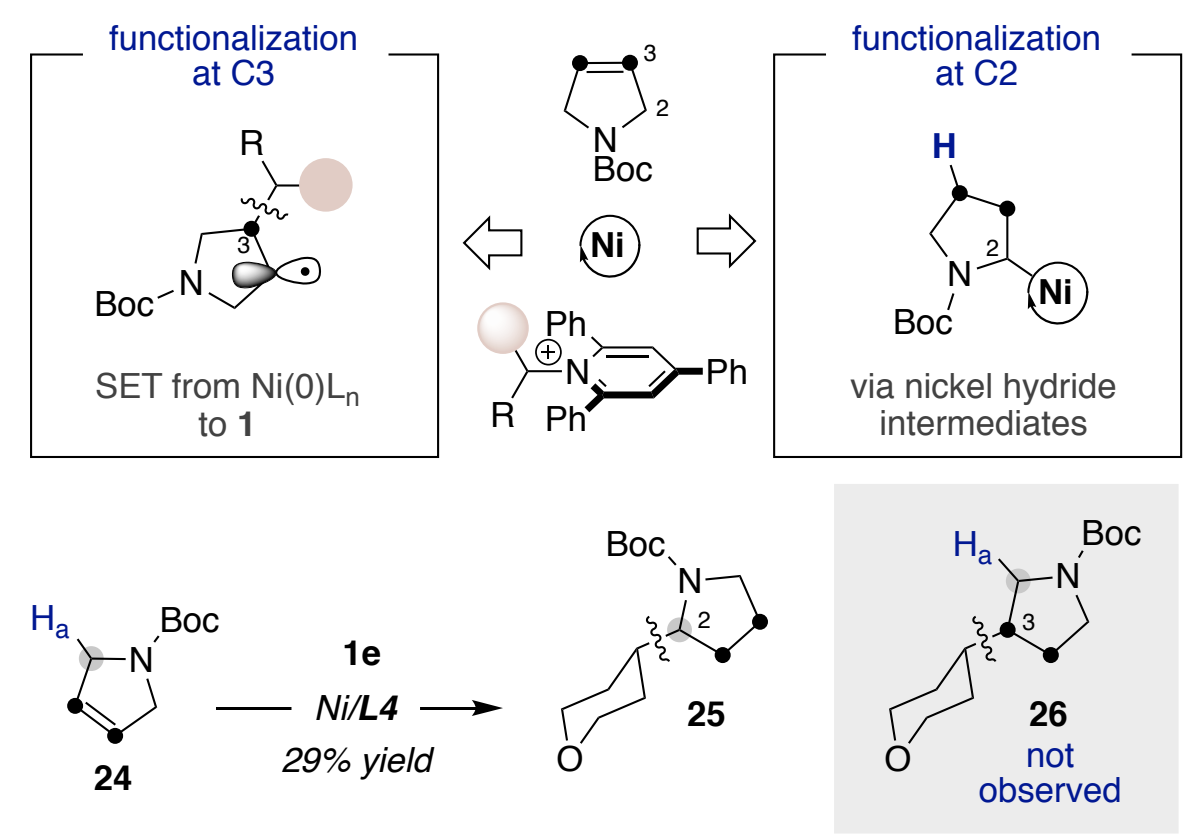

\section{Regioselectivity Studies.}<smiles></smiles><smiles>[18FH]</smiles><smiles>CC(C)(C)OC(=O)N1CC2CC1C2</smiles>

24

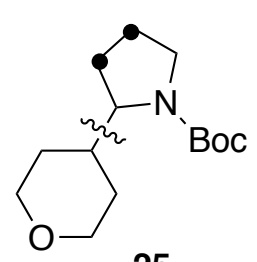

25

An oven-dried $8 \mathrm{~mL}$ screw-cap test tube containing a stirring bar was charged with $\mathrm{NiBr}_{2} \cdot \mathrm{DME}$ (6.2 mg, 10 mol\%), 4,4'-di-tert-butyl-2,2'-dipyridyl (L4, $8.0 \mathrm{mg}, 15 \mathrm{~mol} \%$ ), 
$\mathrm{Na}_{2} \mathrm{HPO}_{4}(56.8 \mathrm{mg}, 0.40 \mathrm{mmol})$ and 2,4,6-triphenyl-1-(tetrahydro-2 $H$-pyran-4-yl)pyridin1-ium tetrafluoroborate $(\mathbf{1 e}, 95.8 \mathrm{mg}, 0.20 \mathrm{mmol})$. Subsequently, the tube was sealed with a Teflon-lined screw cap, then evacuated and back-filled with Ar (3 times). Afterwards, tert-butyl 2,5-dihydro-1H-pyrrole-1-carboxylate (24, $101.4 \mathrm{mg}, 0.6 \mathrm{mmol}),(\mathrm{EtO})_{2} \mathrm{MeSiH}$ (96 $\mu \mathrm{L}, 0.60 \mathrm{mmol}), \mathrm{NMP}$ and 1.4-dioxane $(4: 1,1.0 \mathrm{~mL})$ were added via syringe. Then, the tube was stirred at $40{ }^{\circ} \mathrm{C}$ for $24 \mathrm{~h}$. After the reaction was completed, the mixture was diluted with EtOAc, filtered through silica gel and concentrated under vacuum. The corresponding product 25 were purified by column chromatography on silica gel (Hexane/EtOAc $=10: 1$ to 5:1), affording light yellow oil $15 \mathrm{mg}$ (29\% yield).

An oven-dried $8 \mathrm{~mL}$ screw-cap test tube containing a stirring bar was charged with $\mathrm{NiI}_{2}$ (6.2 mg, $10 \mathrm{~mol} \%$ ), 2-(6-methylpyridin-2-yl)-4,5-dihydrooxazole (L6, $6.4 \mathrm{mg}, 20 \mathrm{~mol} \%$ ), $\mathrm{Na}_{2} \mathrm{HPO}_{4} \quad(113.6 \mathrm{mg}, \quad 0.80 \mathrm{mmol})$ and 2,4,6-triphenyl-1-(tetrahydro-2H-pyran-4yl)pyridin-1-ium tetrafluoroborate (1e, $95.8 \mathrm{mg}, 0.20 \mathrm{mmol})$. Subsequently, the tube was sealed with a Teflon-lined screw cap, then evacuated and back-filled with Ar (3 times). Afterwards, tert-butyl 2,5-dihydro- $1 H$-pyrrole-1-carboxylate (24, $101.4 \mathrm{mg}, 0.6 \mathrm{mmol}, 3.0$ equiv), (EtO) $)_{3} \mathrm{SiH}(74 \mu \mathrm{L}, 0.60 \mathrm{mmol})$, DMSO and 1.4-dioxane $(3: 1,1.2 \mathrm{~mL})$ were added via syringe. Then, the tube was stirred at $35{ }^{\circ} \mathrm{C}$ for $24 \mathrm{~h}$. After the reaction was completed, the mixture was diluted with EtOAc, filtered through silica gel and concentrated under vacuum. The corresponding product $\mathbf{2 5}$ were purified by column chromatography on silica gel (Hexane/EtOAc = $10: 1$ to 5:1), affording light yellow oil $31 \mathrm{mg}$ (61\% yield).

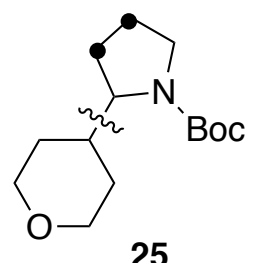

25

tert-buty-2-(tetrahydro-2H-pyran-4-yl)pyrrolidine-1-carboxylate (25) ${ }^{1} \mathrm{H}$ NMR $\left(400 \mathrm{MHz}, \mathrm{CDCl}_{3}\right) \delta 3.92(\mathrm{~d}, J=11.0 \mathrm{~Hz}, 2 \mathrm{H}), 3.75-3.57(\mathrm{~m}, 1 \mathrm{H}), 3.52-3.31(\mathrm{~m}$, 1H), $3.35-3.21(\mathrm{~m}, 2 \mathrm{H}), 3.19-3.04(\mathrm{~m}, 1 \mathrm{H}), 2.08-1.63(\mathrm{~m}, 5 \mathrm{H}), 1.39$ (s, 9H), 1.45 $-1.14(\mathrm{~m}, 4 \mathrm{H}) \mathrm{ppm} .{ }^{13} \mathrm{C}$ NMR $\left(101 \mathrm{MHz}, \mathrm{CDCl}_{3}\right) \delta 155.1,79.2,79.0,68.2,68.0,61.2$, $47.1,46.3,39.1,38.2,28.5,27.9,26.4,24.3,23.4 \mathrm{ppm}$. The observed spectral data are in agreement with the ones reported in literature. ${ }^{5}$

${ }^{5}$ Johnson, C. P.; Smith, R. T.; Allmendinger, S.; MacMillan, D. W. C. Nature, 2016, 535, 322. 


\section{Control Experiments to Explore the Isomerization of 18 in the absence of $1 \mathrm{e}$}

Control experiments were performed to dictate whether olefin isomerization occurred in the absence of pyridinium salts, thus contributing the notion that a nickel hydride is responsible to translocate the active metal species at a different end throughout the sidechain. These experiments were performed with $\mathbf{2 4}$, thus aiming at unraveling the origin of the C2-alkylation product 25 (page S48).

\begin{tabular}{|c|c|c|c|}
\hline Entry & Time & $\begin{array}{c}\text { Yield of } 24 a(\%)^{b} \\
N i / L 4\end{array}$ & $\begin{array}{c}\text { Yield of 24a }(\%)^{b} \\
\text { Ni/L6 }\end{array}$ \\
\hline 1 & $30 \mathrm{~min}$ & 18 & 18 \\
\hline 2 & $1 \mathrm{~h}$ & 17 & 23 \\
\hline 3 & $2 \mathrm{~h}$ & 19 & 23 \\
\hline 4 & $6 \mathrm{~h}$ & 22 & 27 \\
\hline 5 & $12 \mathrm{~h}$ & 25 & 33 \\
\hline 6 & $24 \mathrm{~h}$ & 25 & 40 \\
\hline
\end{tabular}

General procedure for the isomerization of $24 \mathrm{a}$ with $\mathrm{Ni} / \mathrm{L} 4$ system: An oven-dried $8 \mathrm{~mL}$ screw-cap vial containing a stirring bar was charged with $\mathrm{NiBr}_{2} \cdot \mathrm{DME}$ (6.2 mg, 5 mol\%), L4 (8.0 mg, $7.5 \mathrm{~mol} \%), \mathrm{Na}_{2} \mathrm{HPO}_{4}(56.8 \mathrm{mg}, 0.4 \mathrm{mmol})$. Subsequently, the tube was sealed with a Teflon-lined screw cap, then evacuated and backfilled with $\operatorname{Ar}(3$ times), Then, tert-butyl 2,5-dihydro-1H-pyrrole-1-carboxylate (24, $67.6 \mathrm{mg}, 0.4$ mmol), DEMS (96 $\mu$ l, $0.6 \mathrm{mmol}, 1.50$ equiv), NMP and THF (4:1, $1.0 \mathrm{~mL})$ were added by syringe. Then, the tube was stirred at $40{ }^{\circ} \mathrm{C}$ for the indicated time. The yields of $24 \mathbf{a}$ were determined by GC FID taking aliquots using 1-decane as internal standard.

General procedure to form 24a with Ni/L6 systerm: An oven-dried $8 \mathrm{~mL}$ screw-cap vial containing a stirring bar was charged with $\mathrm{NiI}_{2}$ (6.2 mg, 5 mol\%), L6 (6.4 mg, 10 mol\%), $\mathrm{Na}_{2} \mathrm{HPO}_{4}(113.6 \mathrm{mg}, 0.8 \mathrm{mmol})$. Subsequently, the tube was sealed with a Teflon-lined screw cap, then evacuated and backfilled with Ar (3 times).Then, tertbutyl 2,5-dihydro-1H-pyrrole-1-carboxylate (24, $67.6 \mathrm{mg}, 0.4 \mathrm{mmol})$, (EtO) ${ }_{3} \mathrm{SiH}$ (74 $\mu 1,0.40 \mathrm{mmol}, 1.0$ equiv), DMSO and dioxane $(3: 1,1.2 \mathrm{~mL})$ were added via syringe. 
Then, the tube was stirred at $35^{\circ} \mathrm{C}$. The yields of $\mathbf{2 4 a}$ were determined by GC FID taking aliquots using 1-decane as internal standard.

\section{Experiments with radical probe 27.}

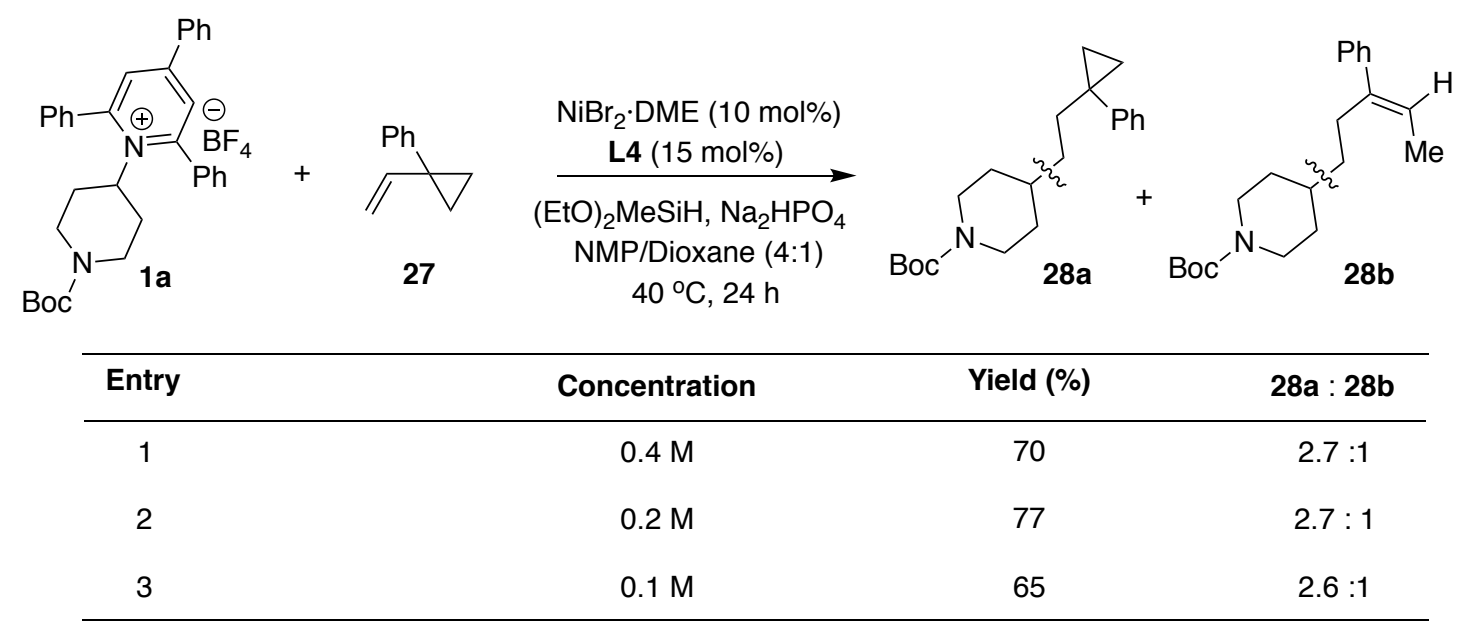

The reaction of 1a with alkenes bearing a proximal cyclopropyl ring such as $\mathbf{2 7}$ is rather illustrative. While a cyclopropylmethyl radical generated upon SET to 1a followed by addition to the olefin should a priori undergo a rapid ring-opening rearrangement, we observed a constant ratio of $\mathbf{2 8 a}$ vs $\mathbf{2 8 b}(2.6: 1)$ at different $\mathrm{Ni} / \mathrm{L}$ loadings, even at particularly low catalyst loadings. Although unravelling the intricacies of these reaction at the molecular level should await further investigations, we tentative believe that these experiments argue against a radical escape rebound mechanism.

General procedure en route to $28 \mathrm{a}$ and $28 \mathrm{~b}$ : An oven-dried $8 \mathrm{~mL}$ screw-cap test tube containing a stirring bar was charged with $\mathrm{NiBr}_{2} \cdot \mathrm{DME}(6.2 \mathrm{mg}, 10 \mathrm{~mol} \%), 4,4^{\prime}$-di-tertbutyl-2,2'-dipyridyl (L4, $8.0 \mathrm{mg}, 15 \mathrm{~mol} \%$ ), $\mathrm{Na}_{2} \mathrm{HPO}_{4}(56.8 \mathrm{mg}, 0.40 \mathrm{mmol})$ and 1-(1-(tertbutoxycarbonyl)piperidin-4-yl)-2,4,6-triphenylpyridin-1-ium tetrafluoroborate (1a, 115.6 $\mathrm{mg}, 0.20 \mathrm{mmol}$ ). Subsequently, the tube was sealed with a Teflon-lined screw cap, then evacuated and back-filled with $\operatorname{Ar}$ (3 times). Afterwards, (1-vinylcyclopropyl) benzene (27, $86.4 \mathrm{mg}, 0.6 \mathrm{mmol})$, (EtO) ${ }_{2} \mathrm{MeSiH}(96 \mu \mathrm{L}, 0.60 \mathrm{mmol})$, NMP and 1.4-dioxane (4:1, 1.0 $\mathrm{mL}$ ) were added via syringe. Then, the tube was stirred at $40^{\circ} \mathrm{C}$ for $24 \mathrm{~h}$. After the reaction was completed, the mixture was diluted with EtOAc, filtered through silica gel and concentrated under vacuum. The corresponding product were purified by column chromatography on silica gel (hexane/EtOAc $=100: 1$ to 40:1), affording colorless oil 28a 
and $28 \mathrm{~b}$ (51 $\mathrm{mg}, 77 \%$ yield) as 2.7:1 mixture (determined by NMR and GC analysis) (colorless oil). ${ }^{1} \mathbf{H}$ NMR (400 MHz, $\left.\mathrm{CDCl}_{3}\right) \delta 7.34-7.24(\mathrm{~m}, 15.59 \mathrm{H}), 7.23-7.13(\mathrm{~m}$, $3.24 \mathrm{H}), 5.73$ (q, $J=6.9 \mathrm{~Hz}, 1 \mathrm{H}), 4.03$ (brs, $7.88 \mathrm{H}), 2.63(\mathrm{~m}, 7.63 \mathrm{H}), 2.55-2.45(\mathrm{~m}, 2.43 \mathrm{H})$, $1.78(\mathrm{~d}, J=6.9 \mathrm{~Hz}, 3.11 \mathrm{H}), 1.70-1.62(\mathrm{~m}, 2.83 \mathrm{H}), 1.60-1.51(\mathrm{~m}, 12.40 \mathrm{H}), 1.45$ (s, 9. H), $1.44(\mathrm{~s}, J=4.9 \mathrm{~Hz}, 23.37 \mathrm{H}), 1.33-1.17(\mathrm{~m}, 15.52 \mathrm{H}), 1.14-0.93(\mathrm{~m}, 8.87 \mathrm{H}), 0.92-$ $0.82(\mathrm{~m}, 5.83 \mathrm{H}), 0.81-0.77(\mathrm{~m}, 5.30 \mathrm{H}), 0.67-0.53(\mathrm{~m}, 5.34 \mathrm{H}) \mathrm{ppm} .{ }^{13} \mathbf{C}$ NMR $(101 \mathrm{MHz}$, $\left.\mathrm{CDCl}_{3}\right) \delta 155.0,145.4,143.3,145.0,129.0,128.3,128.2,126.6,126.3,125.9,122.8,79.3$, 79.2, 44.2, 37.5, 36.2, 36.2, 35.3, 33.93, 28.6, 26.8, 25.8, 22.8, 22.7, 14.2, 14.2, 13.2 ppm. IR (neat, $\mathrm{cm}^{-1}$ ): 2975, 2925, 2850, 1689, 1421, 1365, 1276, 1238, 1160, 1129, 1076, 968, 866. HRMS calcd. for $\left(\mathrm{C}_{21} \mathrm{H}_{31} \mathrm{NNaO}_{2}\right)[\mathrm{M}+\mathrm{H}]^{+}: 352.2247$ found 352.2248. 


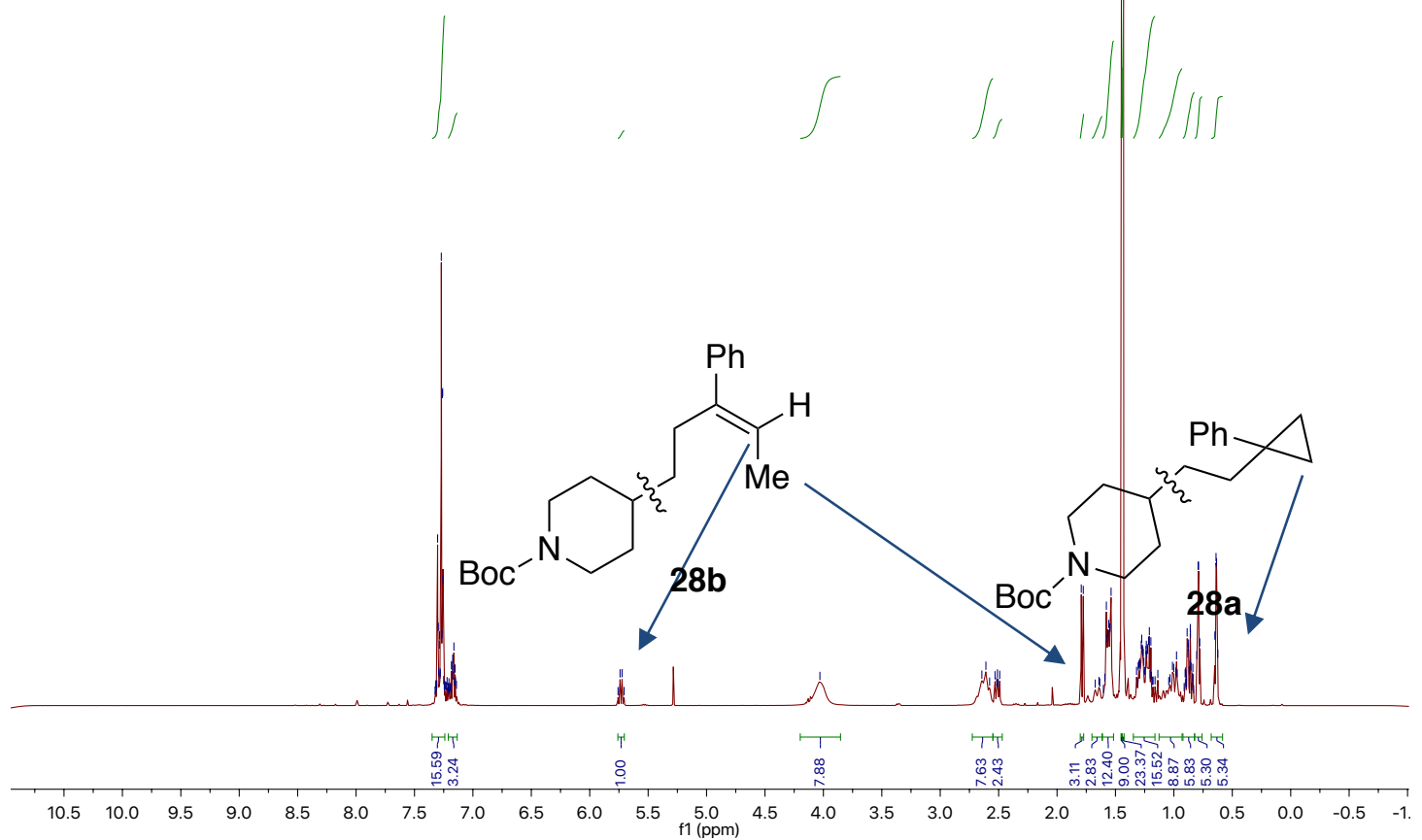

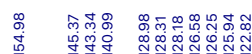

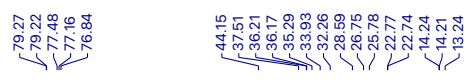<smiles>C/C=C(\CC[C]1CCN(C(=O)OC(C)(C)C)CC1)c1ccccc1</smiles>

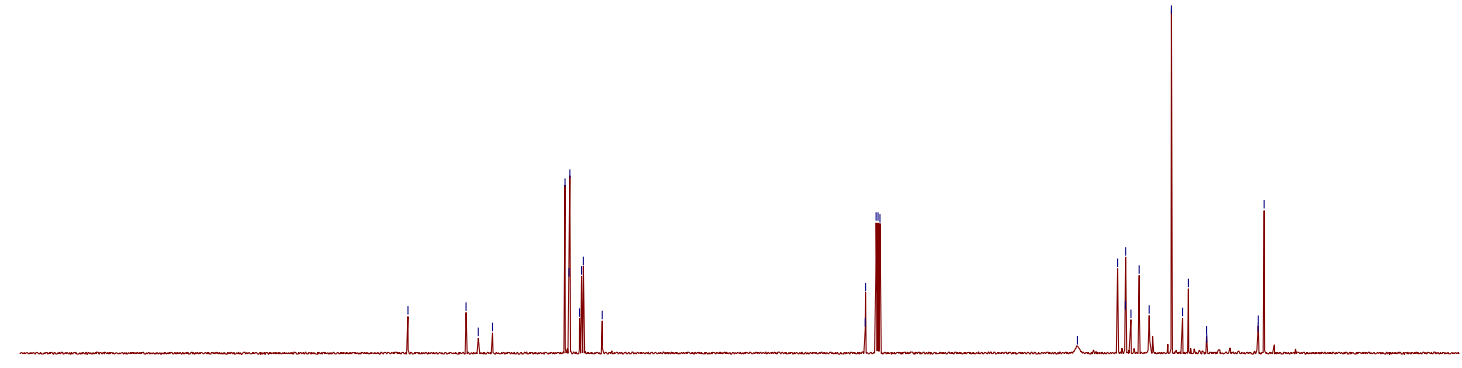

$\begin{array}{lllllllllllllllllllllll}210 & 200 & 190 & 180 & 170 & 160 & 150 & 140 & 130 & 120 & 110 & \underset{f 1}{100}(\mathrm{ppm}) & 90 & 80 & 70 & 60 & 50 & 40 & 30 & 20 & 10 & 0 & -10\end{array}$ 
Stereospecificity of the reaction with pyridinium salts derived from L-alanine<smiles></smiles>

(s)-1n

from L-alanine

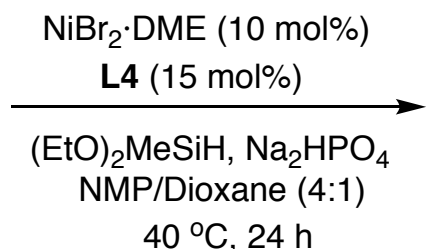

$40^{\circ} \mathrm{C}, 24 \mathrm{~h}$<smiles></smiles>

$29(58 \%)$

racemic

An oven-dried $8 \mathrm{~mL}$ screw-cap test tube containing a stirring bar was charged with $\mathrm{NiBr}_{2} \cdot \mathrm{DME}$ (6.2 mg, 10 mol\%), 4,4'-di-tert-butyl-2,2'-dipyridyl (L4, $8.0 \mathrm{mg}, 15 \mathrm{~mol} \%$ ), $\mathrm{Na}_{2} \mathrm{HPO}_{4}$ (56.8 mg, $0.40 \mathrm{mmol}$ ) and (S)-1-(1-methoxy-1-oxopropan-2-yl)-2,4,6triphenylpyridin-1-ium tetrafluoroborate $(\mathbf{1 n}, 96.2 \mathrm{mg}, 0.20 \mathrm{mmol})$. Subsequently, the tube was sealed with a Teflon-lined screw cap, then evacuated and back-filled with Ar (3 times). Afterwards, tert-butyl(hex-5-en-1-yloxy)dimethylsilane (2c, 128.4 mg, 0.6 mmol), (EtO) $)_{2} \mathrm{MeSiH}(96 \mathrm{uL}, 0.60 \mathrm{mmol}), \mathrm{NMP}$ and 1.4-dioxane (4:1, $\left.1.0 \mathrm{~mL}\right)$ were added via syringe. Then, the tube was stirred at $40{ }^{\circ} \mathrm{C}$ for $24 \mathrm{~h}$. After the reaction was completed, the mixture was diluted with EtOAc, filtered through silica gel and concentrated under vacuum. The corresponding product were purified by column chromatography on silica gel (toluene to hexane/EtOAc $=20: 1$ ), affording colorless oil 29 ( $35 \mathrm{mg}, 58 \%$ yield). The enantiomeric excess was determined to be $\sim 9 \%$ by chiral HPLC analysis (CHIRALPAK IB $(100 \times 4.6 \mathrm{~mm}, 3 \mathrm{u}), 3.0 \mathrm{~mL} / \mathrm{min}, \mathrm{CO}_{2} / \mathrm{MeCN}=90: 10$, $\lambda=210 \mathrm{~nm})$; tR $($ enantiomer $\mathrm{A})=6.02 \mathrm{~min}$, $\mathrm{tR}($ enantiomer $\mathrm{B})=6.54 \mathrm{~min} .{ }^{1} \mathbf{H} \mathbf{~ N M R}$ $\left(400 \mathrm{MHz}, \mathrm{CDCl}_{3}\right) \delta 3.66(\mathrm{~s}, 3 \mathrm{H}), 3.58(\mathrm{t}, J=6.6 \mathrm{~Hz}, 2 \mathrm{H}), 2.53-2.32(\mathrm{~m}, 1 \mathrm{H}), 1.70-$ $1.58(\mathrm{~m}, 1 \mathrm{H}), 1.53-1.45(\mathrm{~m}, 2 \mathrm{H}), 1.44-1.35(\mathrm{~m}, 1 \mathrm{H}), 1.31-1.21(\mathrm{~m}, 6 \mathrm{H}), 1.13(\mathrm{~d}, J$ $=7.0 \mathrm{~Hz}, 3 \mathrm{H}), 0.88(\mathrm{~s}, 9 \mathrm{H}), 0.03(\mathrm{~s}, 6 \mathrm{H}) \mathrm{ppm} .{ }^{13} \mathbf{C ~ N M R}\left(101 \mathrm{MHz}, \mathrm{CDCl}_{3}\right) \delta 177.5$, 63.4, 51.6, 39.6, 33.9, 32.9, 29.4, 27.4, 26.1, 25.8, 18.5, 17.2, -5.1 ppm. IR (neat, cm 1): 2930, 2857, 1739, 1433, 1253, 1195, 1162, 1095, 834, 714. HRMS calcd. for $\left(\mathrm{C}_{16} \mathrm{H}_{35} \mathrm{O}_{3} \mathrm{Si}\right)[\mathrm{M}+\mathrm{H}]^{+}: 303.2350$ found 303.2352 . 


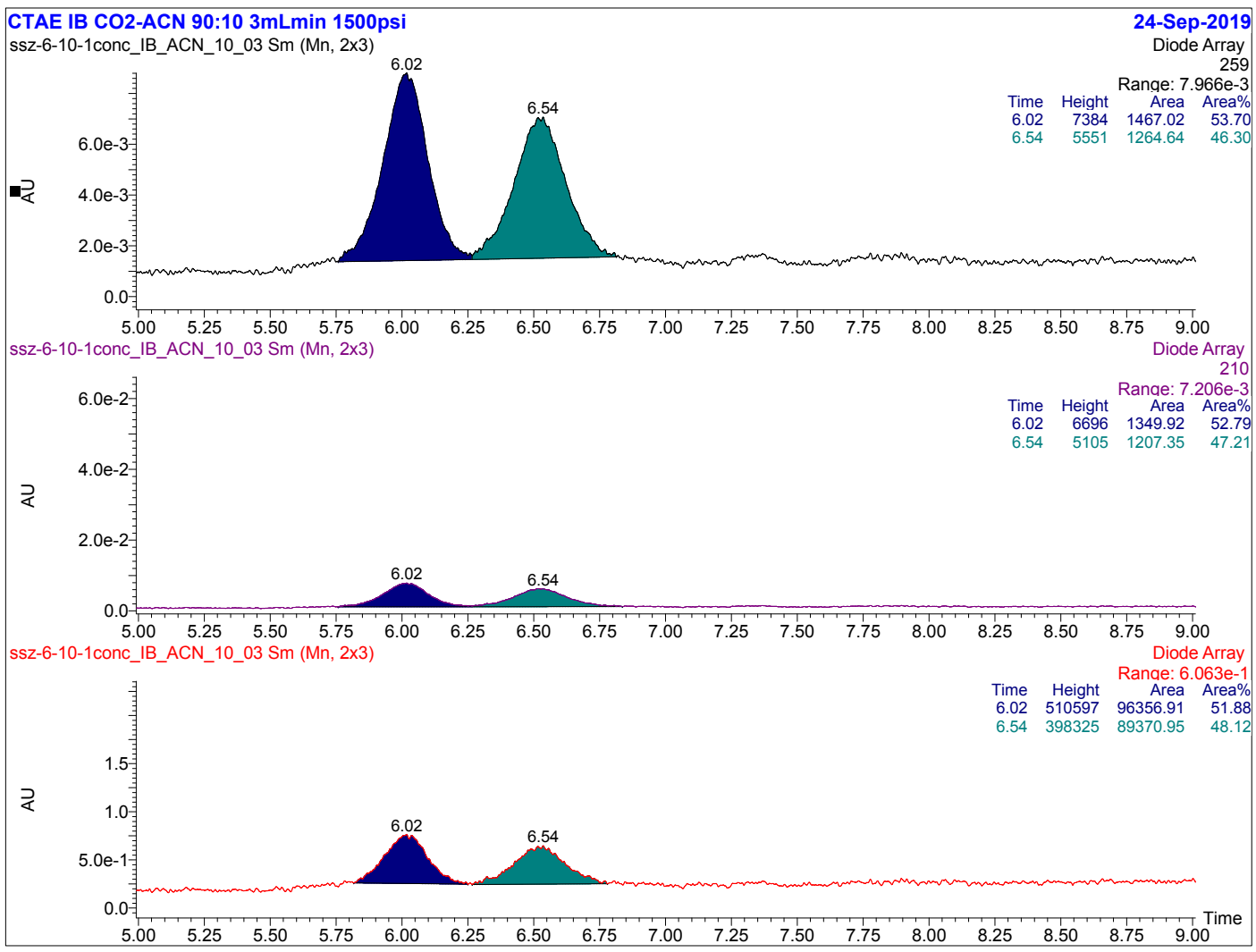




\section{References:}

1. Sun, S.-Z.; Borjesson, M.; Martin-Montero, R.; Martin, R. J. Am. Chem. Soc., 2018, 140,12765 .

2. Martin-Montero, R.; Yatham, V. R.; Yin, H.; Davies, J.; Martin, R. Org. Lett., 2019, 21, 2947.

3. Plunkett, S.; Basch, C. H.; Santana, S. O.; Watson, M. P. J. Am. Chem. Soc., 2019, $141,2257$.

4. Klauck, F. J. R.; James, M. J.; Glorius, F. Angew. Chem. Int. Ed. 2017, 56, 12336.

5. Hoerrner, M. E.; Baker, K. M.; Basch, C. H.; Bampo, E. M.; Watson, M. P. Org. Lett. 2019, 21, 7356.

6. Bag, S.; Jayarajan, R.; Mondal, R.; Maiti, D. Angew. Chem. Int. Ed. 2017, 56, 3182.

7. Ho, G.-M.; Judkele, L.; Bruffaerts, J.; Marek, I. Angew. Chem. Int. Ed. 2018, 57, 8012.

8. Yang, T.; Lu, L.; Shen, Q. Chem. Commun., 2015, 51, 5479.

9. Yoshida, M.; Saito, K.; Kato, Hikaru.; Tsukamoto, S.; Doi, T. Angew. Chem. Int. Ed. 2018, $57,5147$.

10. Gao, D.-W.; Vinogradova, E. V.; Nimmagadda, S. K.; Medina, J. M.; Xiao, Y.; Suciu, R. M.; Cravatt, B. F.; Engle, K. M. J. Am. Chem. Soc., 2018, 140, 8069.

11. L. Gonnard, A. Guerinot, J. Cossy, Chem. Eur, J. 2015, 21, 12797.

12. Wang, Y.-X; Wang, J.-H.; Li, G.-X.; He, G.; Chen, G. Org. Lett. 2017, 19, 1442.

13. Kuhn, K. M.; Champagne, T. M.; Hong, S. H.; Wei, W-H.; Nickel, A.; Lee, C. W.;

Virgil, S. C.; Grubbs, R. H.; Pederson, R. L. Org. Lett., 2010, 12, 984.

14. Gandon, V.; Bertus, P.; Szymoniak, J. Eur. J. Org. Chem. 2000, 22, 3713. 
${ }^{1} \mathrm{H}$ NMR and ${ }^{13} \mathrm{C}$ NMR Spectra:

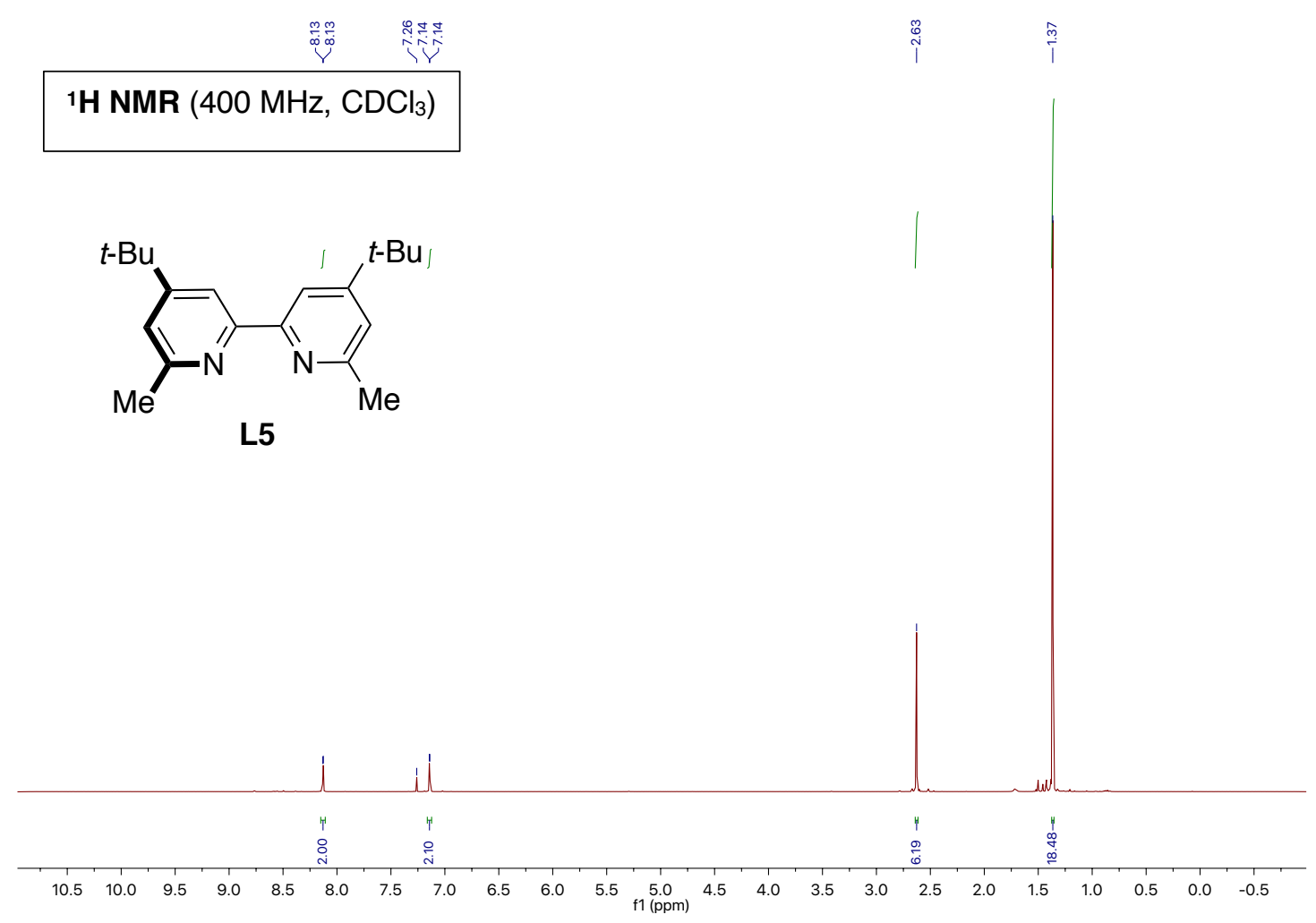

\section{部融}

${ }^{13} \mathrm{C}$ NMR $\left(101 \mathrm{MHz}, \mathrm{CDCl}_{3}\right)$

1

|ن

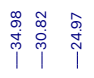<smiles>Cc1cc(-c2cc(C(C)(C)C)cc(C)n2)cc(C)n1</smiles>

L5

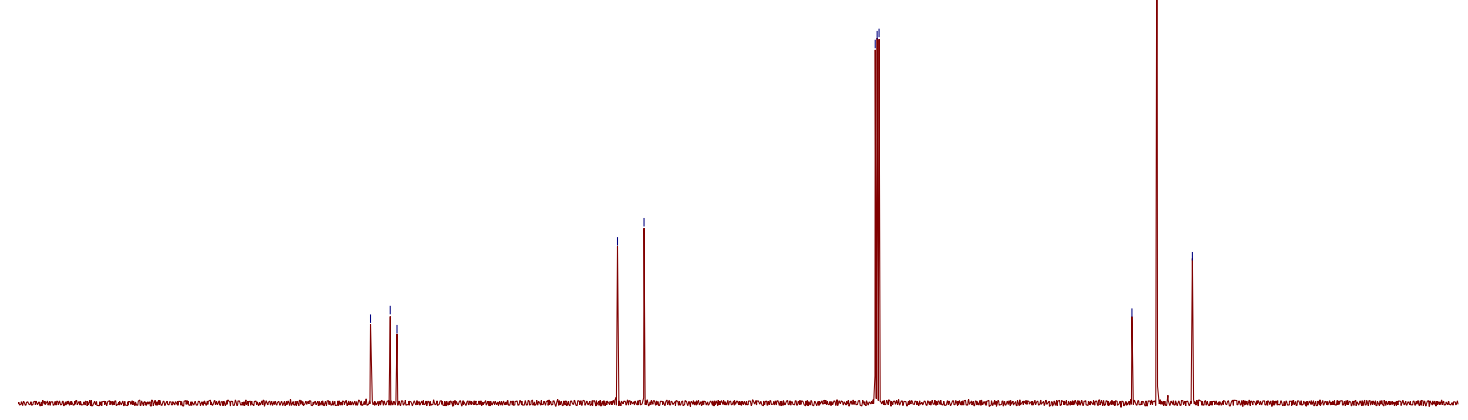

$\begin{array}{llllllllllllllllllllllllllllll}210 & 200 & 190 & 180 & 170 & 160 & 150 & 140 & 130 & 120 & 110 & 100 & 90 & 80 & 70 & 60 & 50 & 40 & 30 & 20 & 10 & 0 & -10\end{array}$ 


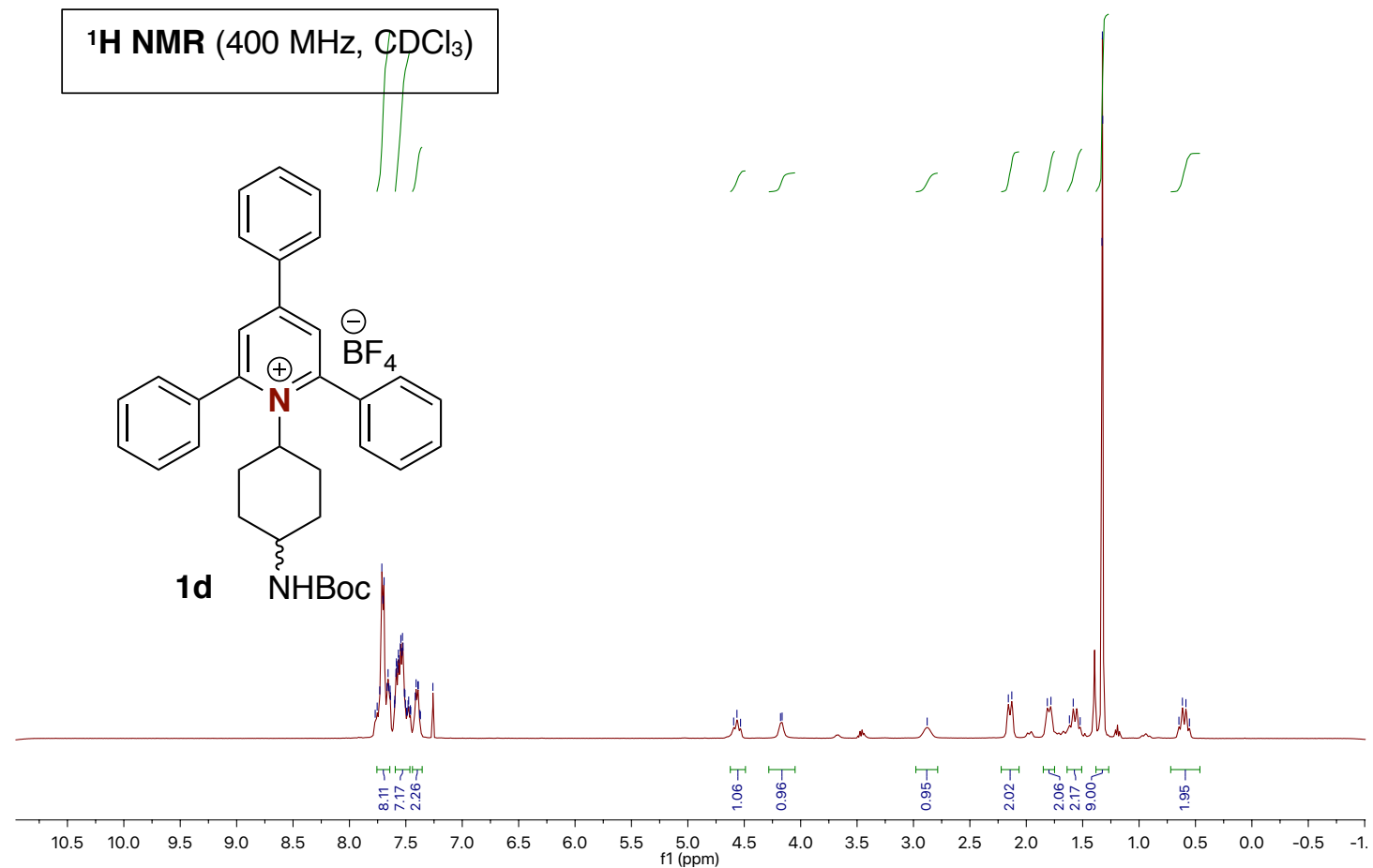

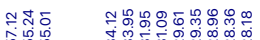

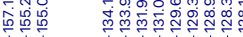

${ }^{13} \mathrm{C}$ NMR $\left(101 \mathrm{MHz}, \mathrm{CDCl}_{3}\right)$

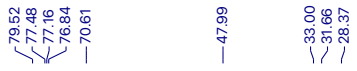<smiles></smiles>

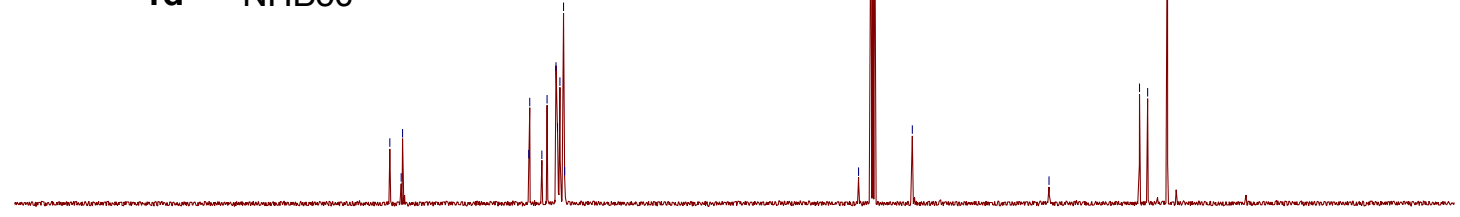

$\begin{array}{llllllllllllllllllllllllll}210 & 200 & 190 & 180 & 170 & 160 & 150 & 140 & 130 & 120 & 110 & 100 & 90 & 80 & 70 & 60 & 50 & 40 & 30 & 20 & 10 & 0 & -10\end{array}$ 
19F NMR (376 MHz, $\left.\mathrm{CDCl}_{3}\right)$<smiles></smiles>

$\begin{array}{llllllllllllllllllllllllllllllllllll}30 & 20 & 10 & 0 & -10 & -20 & -30 & -40 & -50 & -60 & -70 & -80 & -90 & -100 & -110 & -120 & -130 & -140 & -150 & -160 & -170 & -180 & -190 & -200 & -210 & -220 & -230\end{array}$

11B NMR (128 MHz, $\left.\mathrm{CDCl}_{3}\right)$<smiles></smiles>

\begin{tabular}{llllllllllllllllllllllllllllll}
\hline & 0 & -10 & -20 & -30 & -40 & -50 & -60 & -70 & -80 & -90 & -100 & -110 & -120 & -130 & -140 & 150 & -160 & -170 & -180 & -190 & -200 & -210 & -220 & -230 & -240
\end{tabular} 


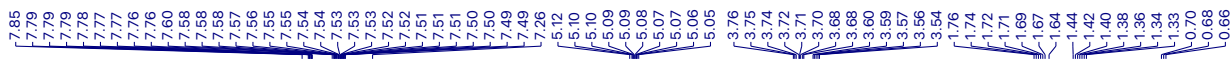
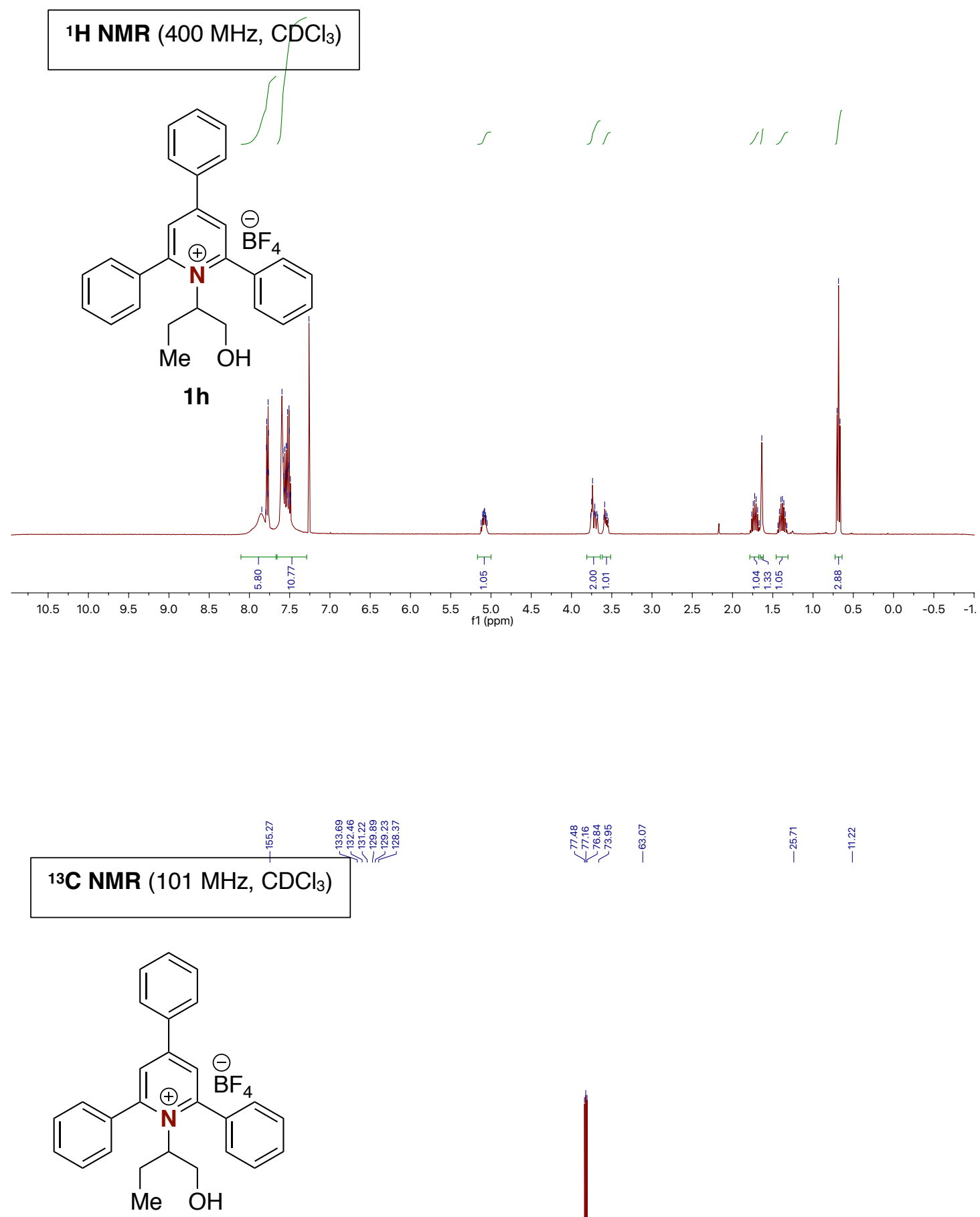

$1 \mathrm{~h}$

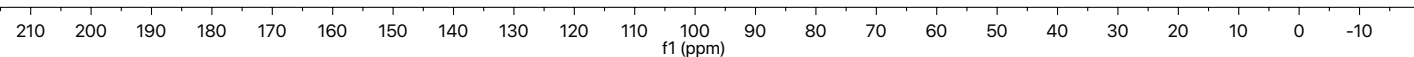


19F NMR (376 MHz, $\left.\mathrm{CDCl}_{3}\right)$<smiles></smiles>

$1 \mathrm{~h}$

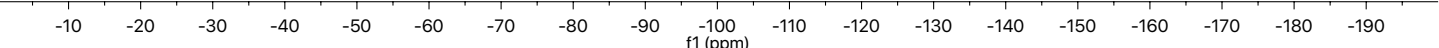

11B NMR (128 MHz, $\left.\mathrm{CDCl}_{3}\right)$

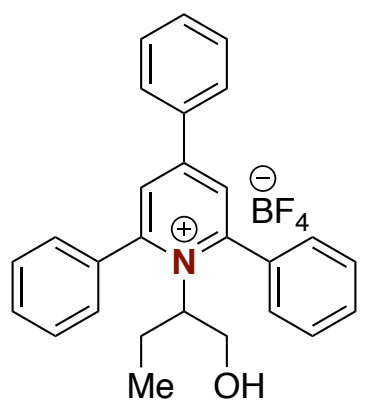

$1 \mathrm{~h}$

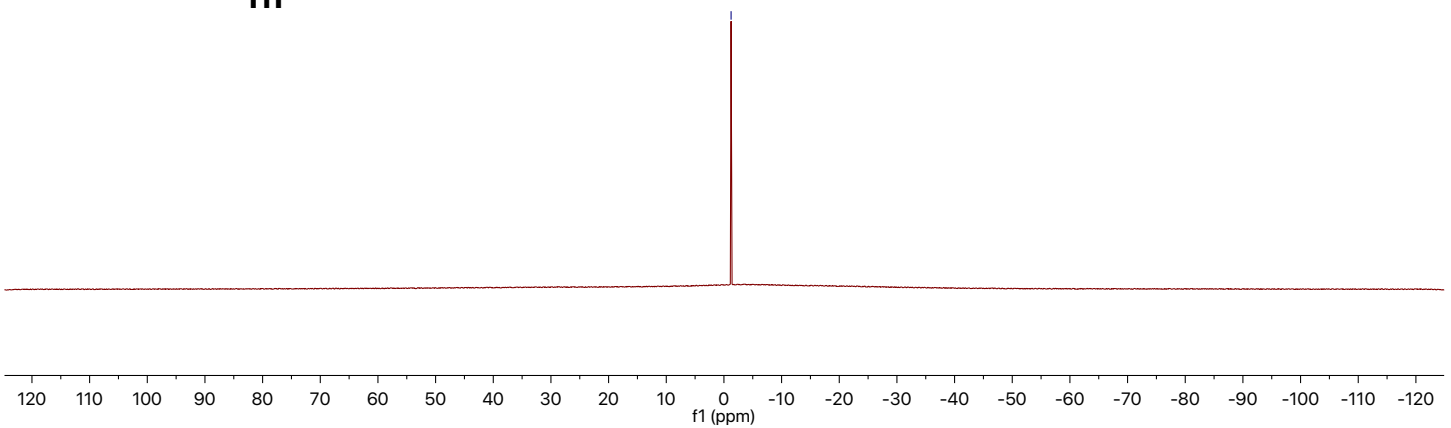


管

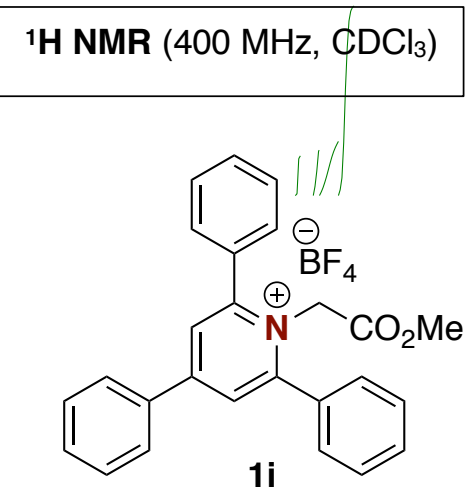

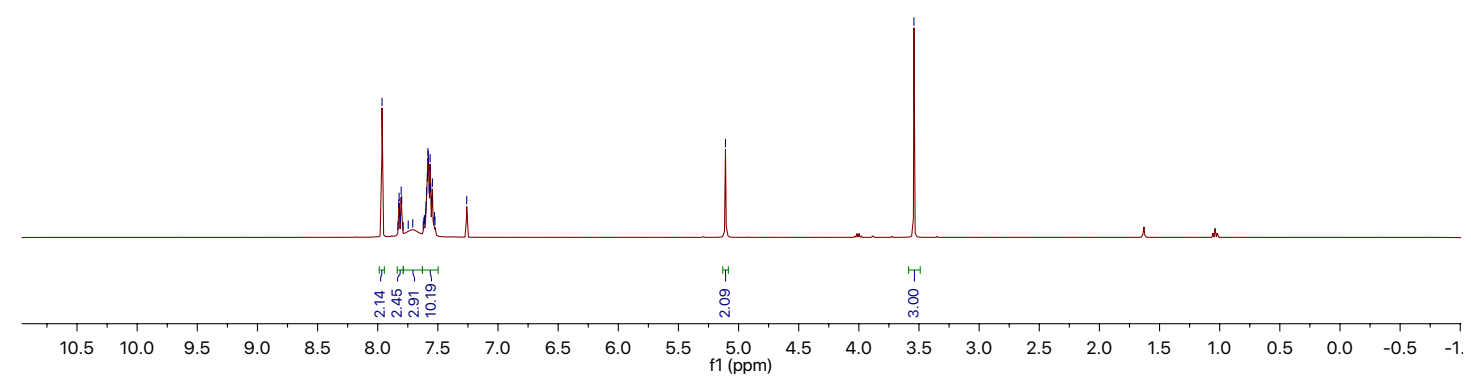

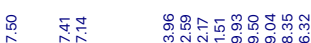

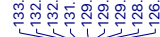

$\left.{ }^{13} \mathrm{C} \mathrm{NMR} \mathrm{(101} \mathrm{MHz,} \mathrm{CDCl}_{3}\right)$

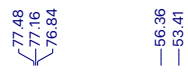<smiles>CC(=O)C[n+]1c(-c2ccccc2)cc(-c2ccccc2)cc1-c1ccccc1</smiles>

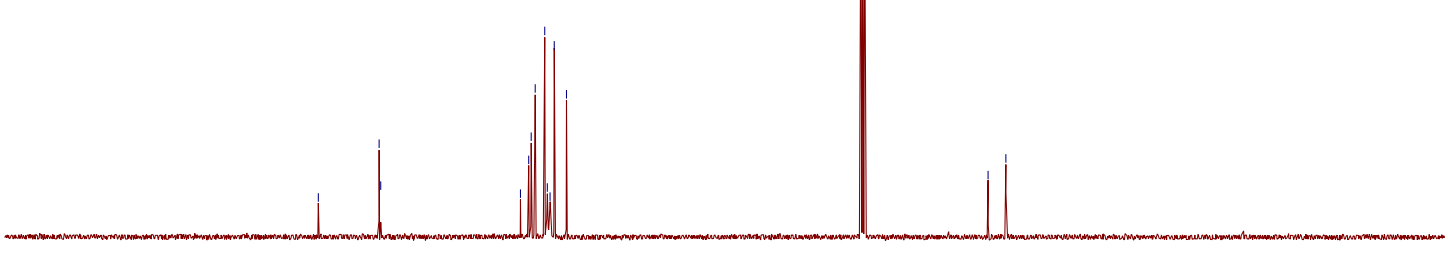

$\begin{array}{lllllllllllllllllllllll}210 & 200 & 190 & 180 & 170 & 160 & 150 & 140 & 130 & 120 & 110 & 100 & 90 & 80 & 70 & 60 & 50 & 40 & 30 & 20 & 10 & 0 & -10\end{array}$ 
19F NMR (376 MHz, $\left.\mathrm{CDCl}_{3}\right)$<smiles></smiles>

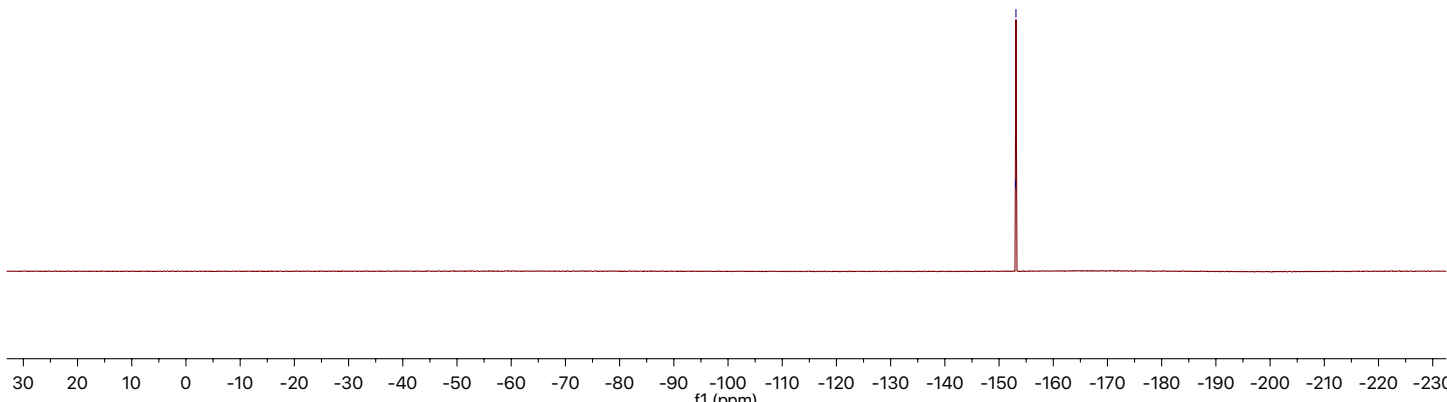

11B NMR (128 MHz, $\left.\mathrm{CDCl}_{3}\right)$<smiles></smiles>

$\begin{array}{lllllllllllllllllllllllllllllllllllll}0 & -10 & -20 & -30 & -40 & -50 & -60 & -70 & -80 & -90 & -100 & -110 & -120 & -130 & -140 & -150 & -160 & -170 & -180 & -190 & -200 & -210 & -220 & -230 & -240\end{array}$ 

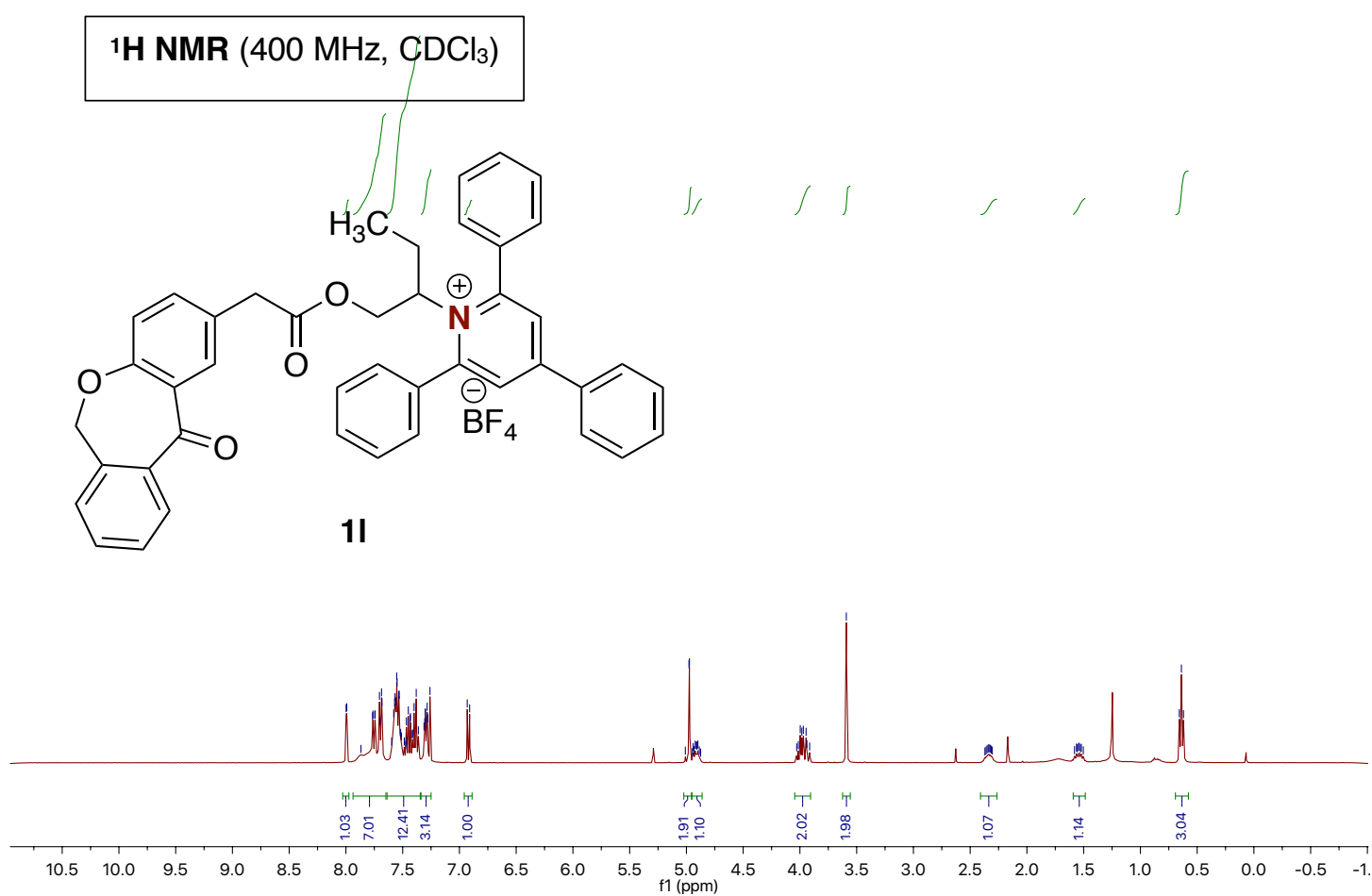

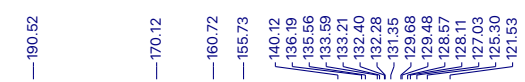

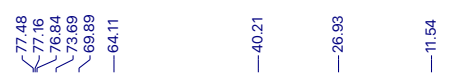

$\left.{ }^{13} \mathrm{C} \mathrm{NMR} \mathrm{(101} \mathrm{MHz,} \mathrm{CDCl}_{3}\right)$

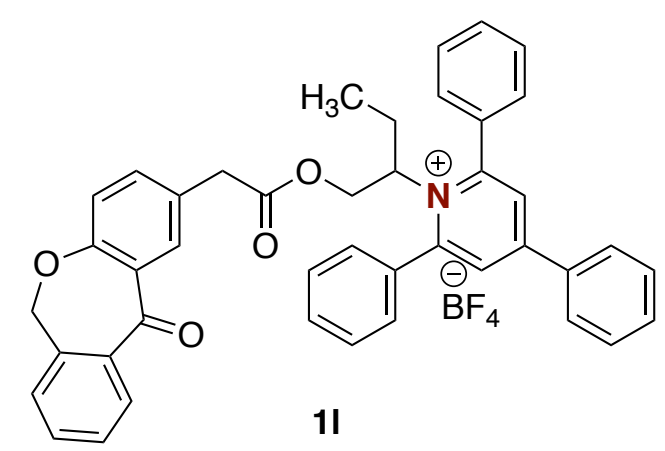

$\begin{array}{llllllllllll}210 & 200 & 190 & 180 & 170 & 160 & 150 & 140 & 130 & 120 & 110 & 100 \\ \mathrm{f} 1(\mathrm{ppm}) & 90\end{array}$ 
19F NMR (376 MHz, $\left.\mathrm{CDCl}_{3}\right)$<smiles></smiles>

11B NMR (128 MHz, $\left.\mathrm{CDCl}_{3}\right)$<smiles></smiles>

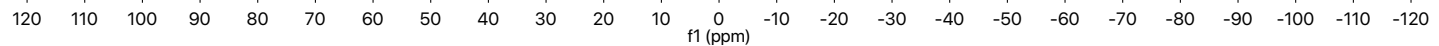




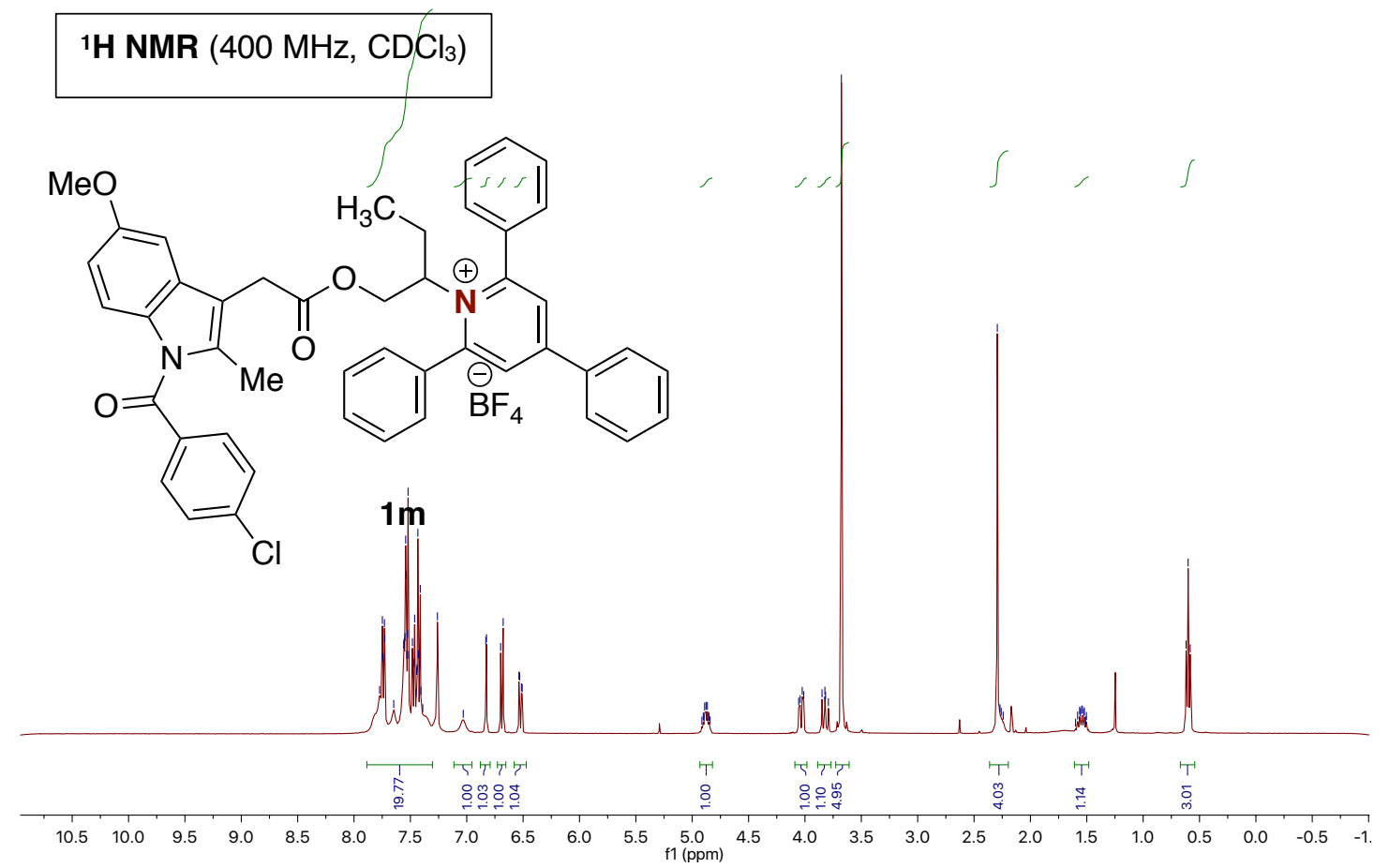

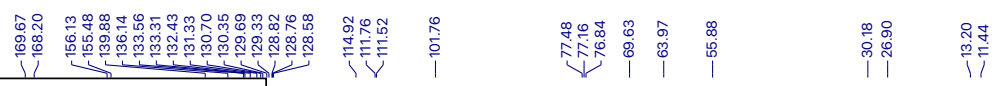

${ }^{13} \mathrm{C}$ NMR $\left(101 \mathrm{MHz}, \mathrm{CDCl}_{3}\right)$
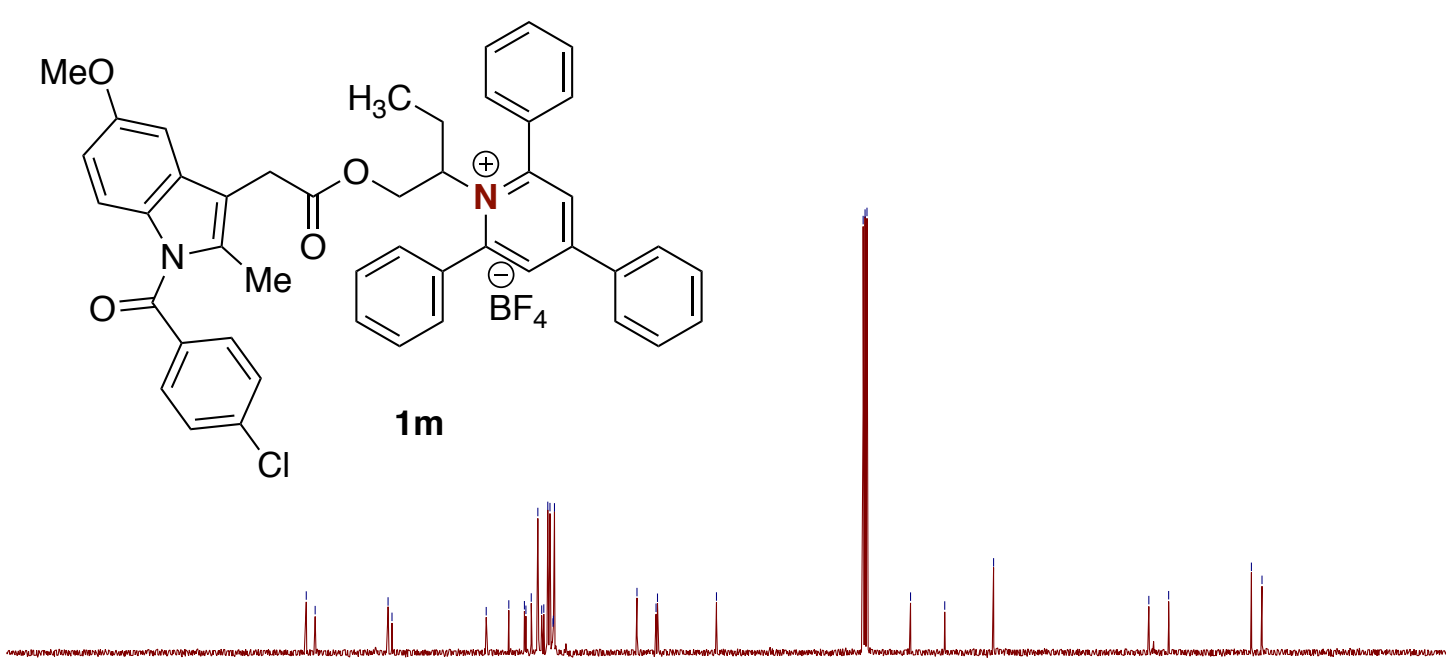

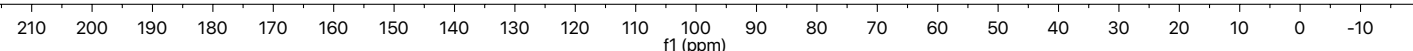


19F NMR (376 MHz, $\left.\mathrm{CDCl}_{3}\right)$

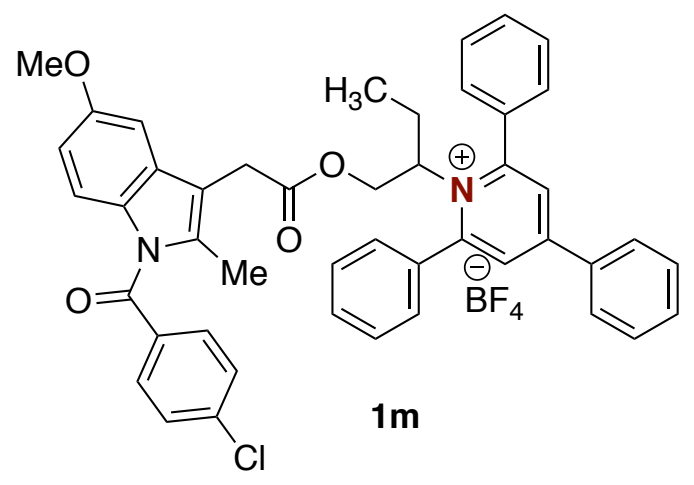

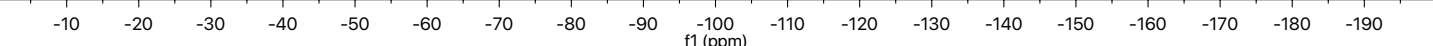

$\stackrel{\infty}{i}$

11B NMR (128 MHz, $\left.\mathrm{CDCl}_{3}\right)$

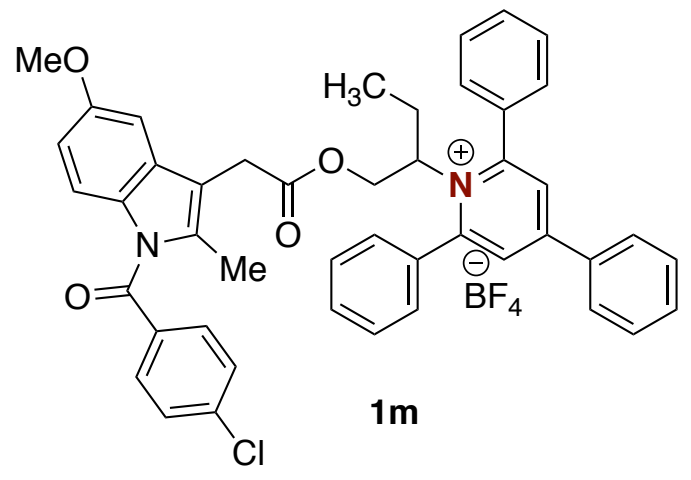

\begin{tabular}{llllllllllllllllllllllllll}
\hline 120 & 110 & 100 & 90 & 80 & 70 & 60 & 50 & 40 & 30 & 20 & 10 & 0 & 0 & -10 & -20 & -30 & -40 & -50 & -60 & -70 & -80 & -90 & -100 & -110 & -120
\end{tabular} 


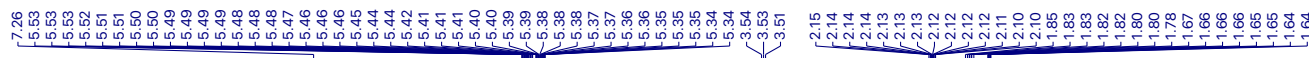

1H NMR (400 MHz, $\left.\mathrm{CDCl}_{3}\right)$
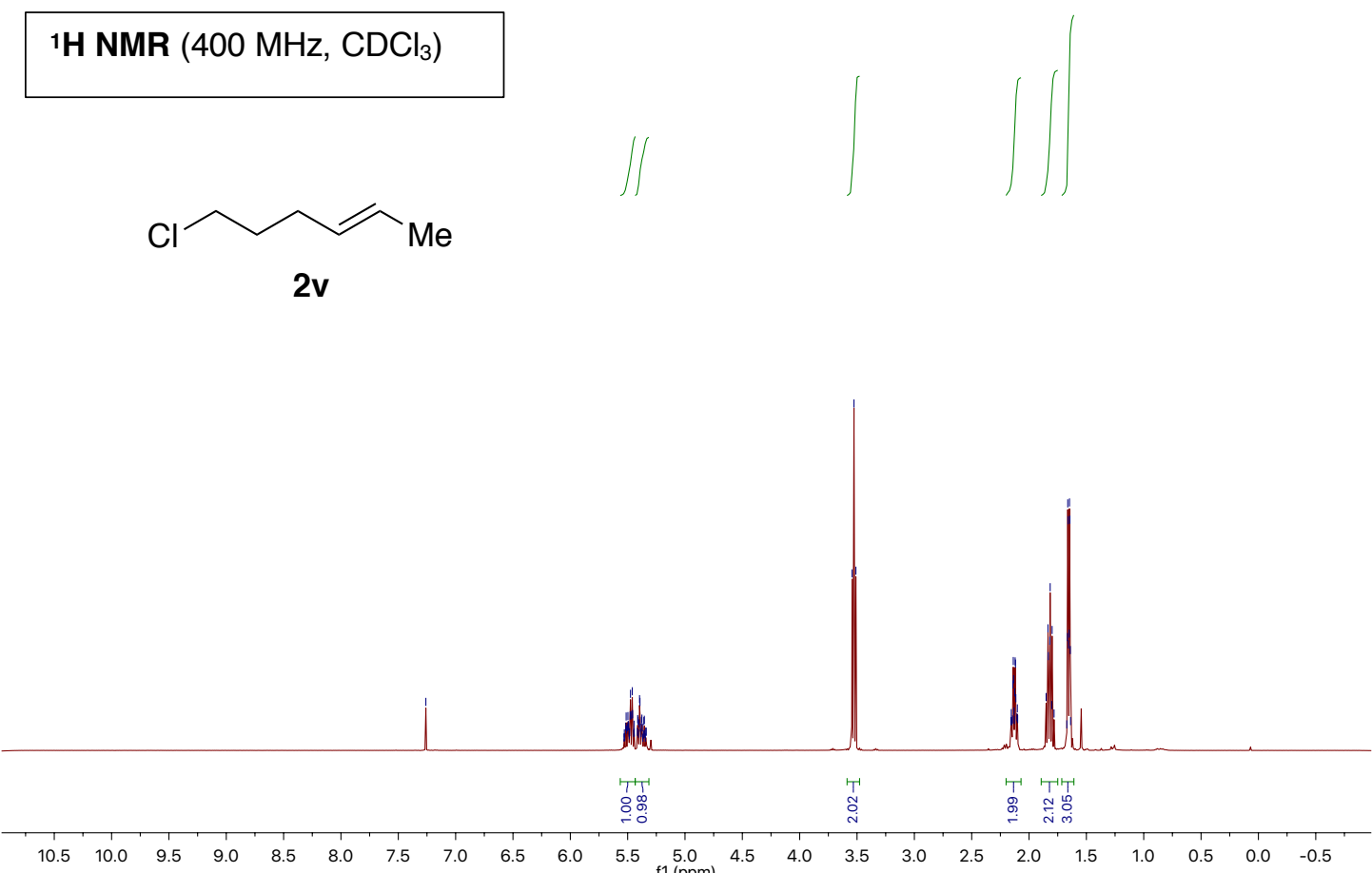

${ }^{13} \mathrm{C}$ NMR $\left(101 \mathrm{MHz}, \mathrm{CDCl}_{3}\right)$

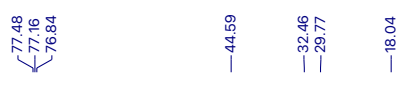

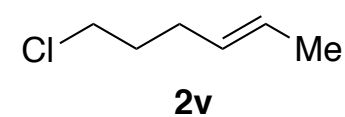

| 


\section{H NMR $\left(400 \mathrm{MHz}, \mathrm{CDCl}_{3}\right)$}

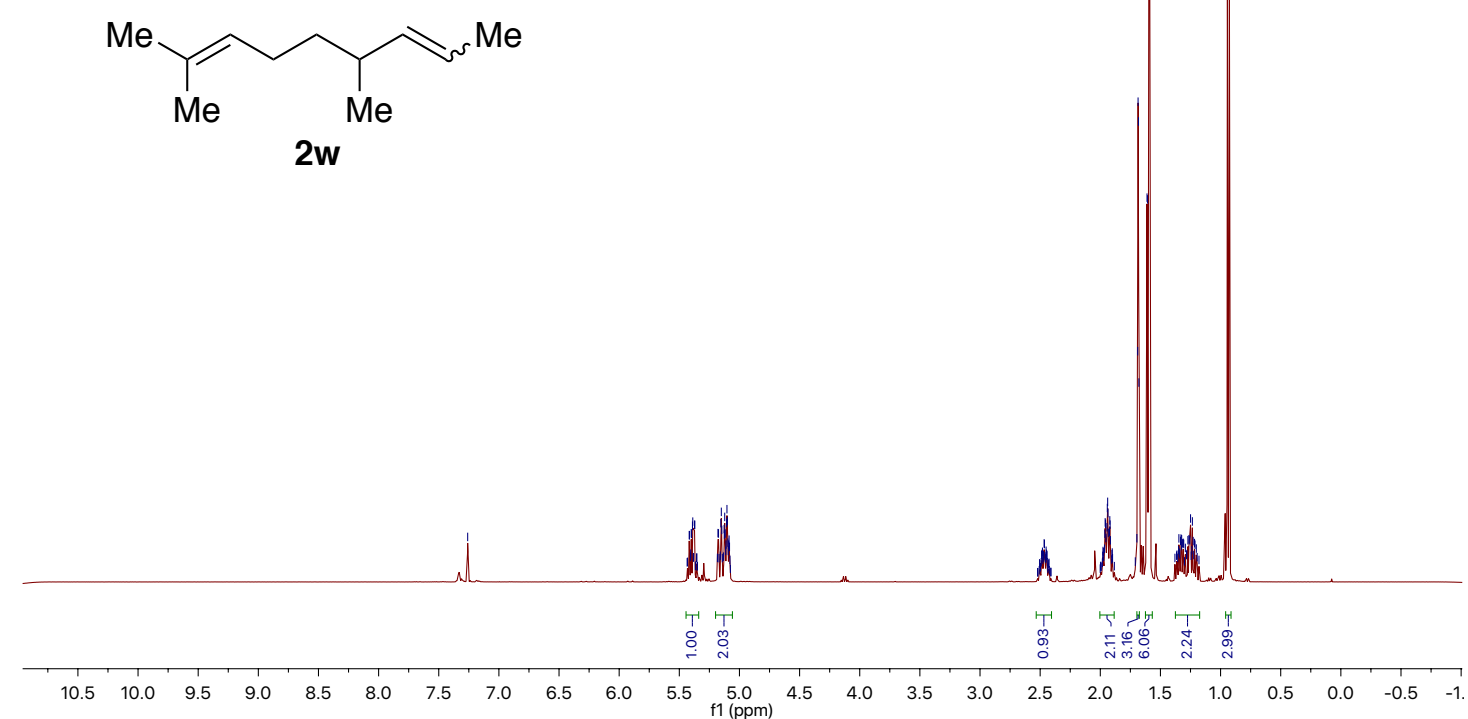

${ }^{13} \mathrm{C}$ NMR $\left(101 \mathrm{MHz}, \mathrm{CDCl}_{3}\right)$<smiles>CC=CC(C)CCC=C(C)C</smiles>

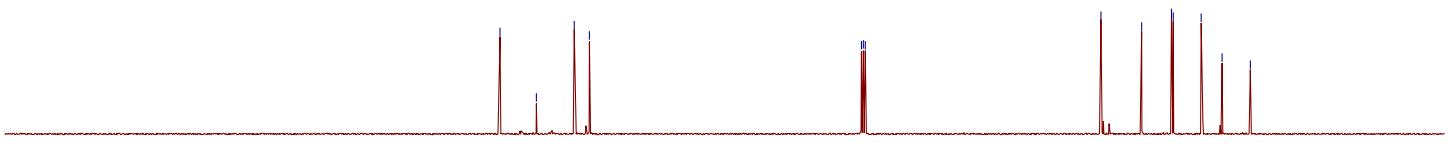

$\begin{array}{lllllllllllllllllllllllllll}210 & 200 & 190 & 180 & 170 & 160 & 150 & 140 & 130 & 120 & 110 & 100 & 90 & 80 & 70 & 60 & 50 & 40 & 30 & 20 & 10 & 0 & -10 & \end{array}$ 


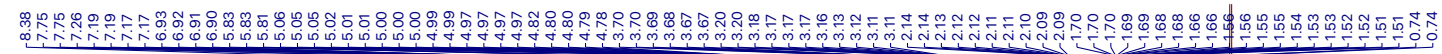

1 $\mathbf{H}$ NMR $\left(400 \mathrm{MHz}, \mathrm{CDCl}_{3}\right)$<smiles>C=CCCCCO[C@H](Cc1ccc(Cl)cc1)n1cncn1</smiles>

2ad

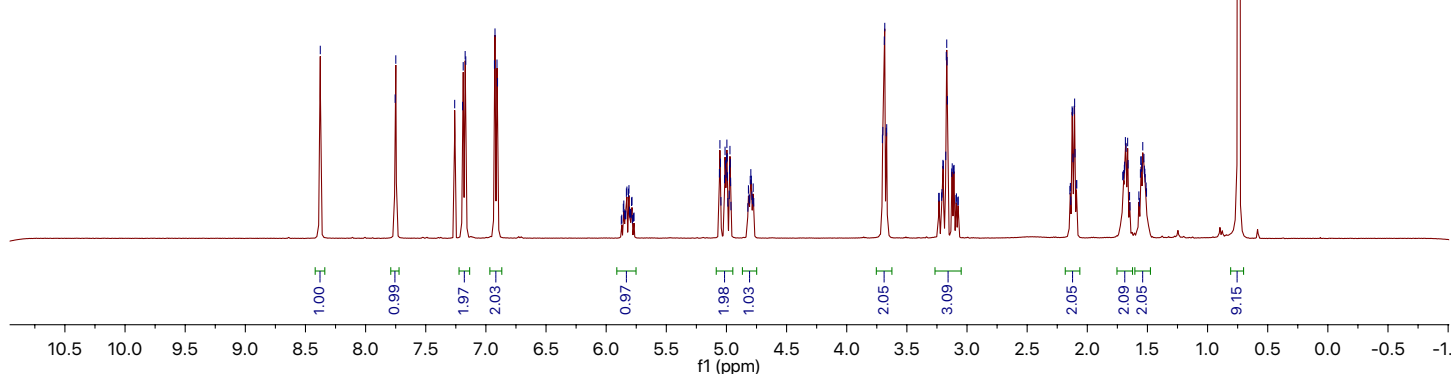

${ }^{13} \mathrm{C}$ NMR (101 MHz, $\left.\mathrm{CDCl}_{3}\right)$<smiles>C=CCCCCO[C@H](C(Cc1ccc(Cl)cc1)n1cncn1)C(C)(C)C</smiles>

2ad

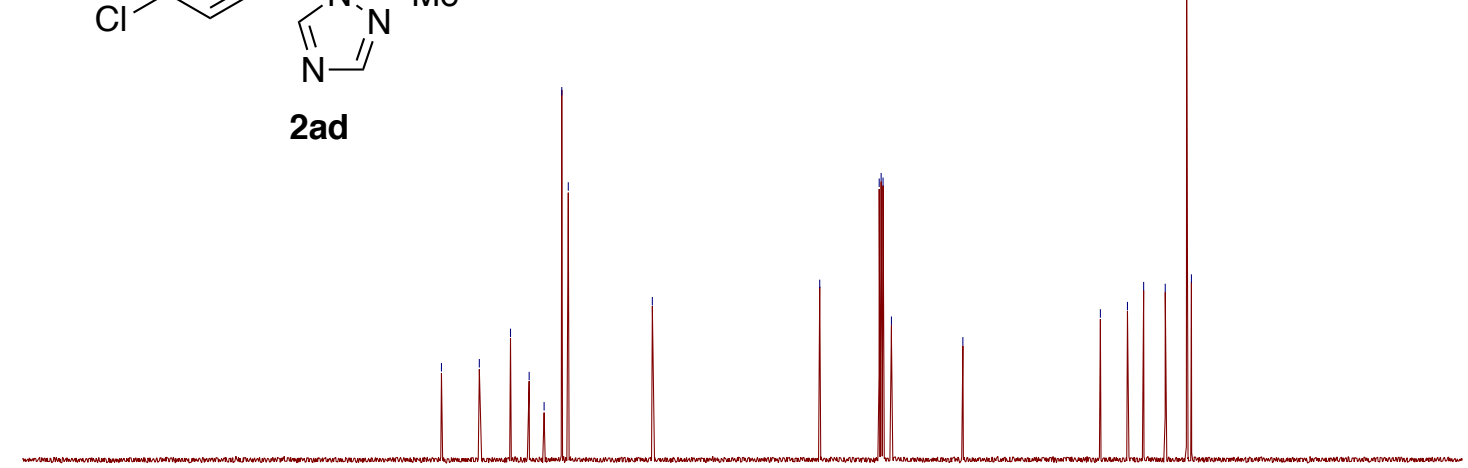




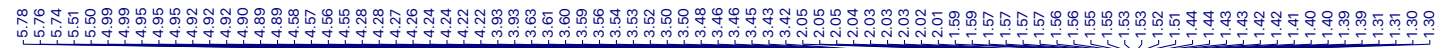
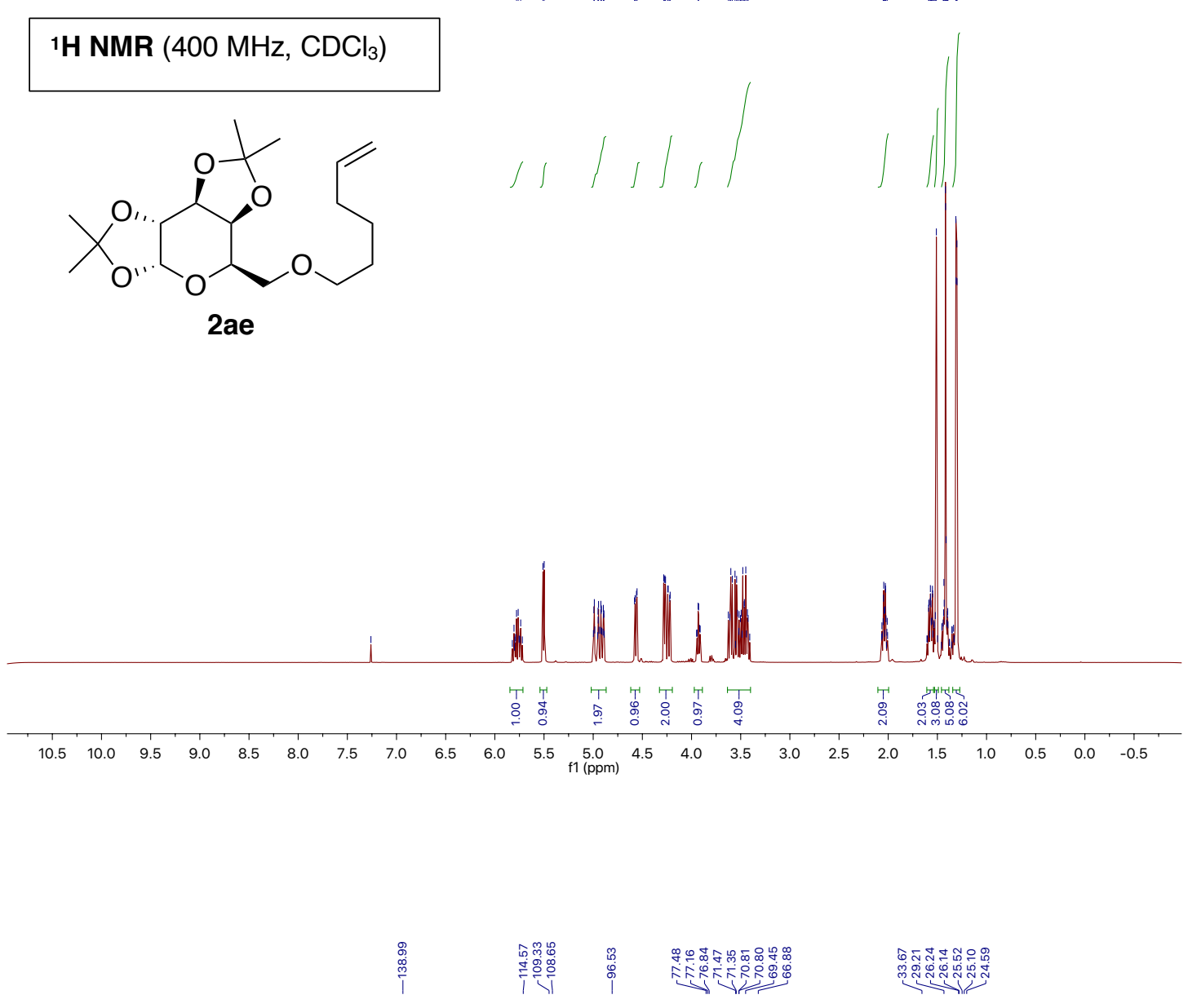

${ }^{13} \mathrm{C} \mathrm{NMR}\left(101 \mathrm{MHz}, \mathrm{CDCl}_{3}\right)$<smiles>C=CCCCOC[C@H]1O[C@@H]2OC(C)(C)O[C@H]2[C@H]2OC(C)(C)O[C@@H]21</smiles>

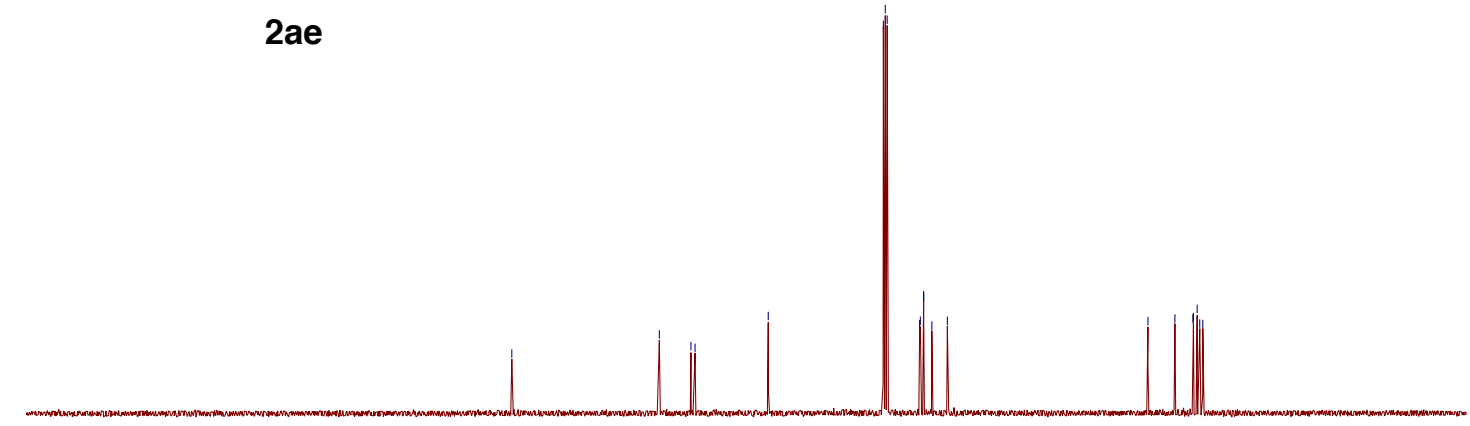

$\begin{array}{lllllllllllllllllllllllll}210 & 200 & 190 & 180 & 170 & 160 & 150 & 140 & 130 & 120 & 110 & \begin{array}{c}100 \\ \mathrm{f} 1(\mathrm{pm})\end{array} & 90 & 80 & 70 & 60 & 50 & 40 & 30 & 20 & 10 & 0 & -10\end{array}$ 
1H NMR (400 MHz, $\left.\mathrm{CDCl}_{3}\right)$

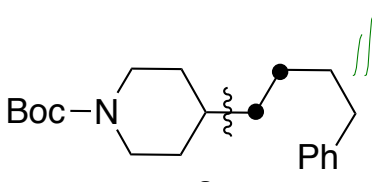

3a

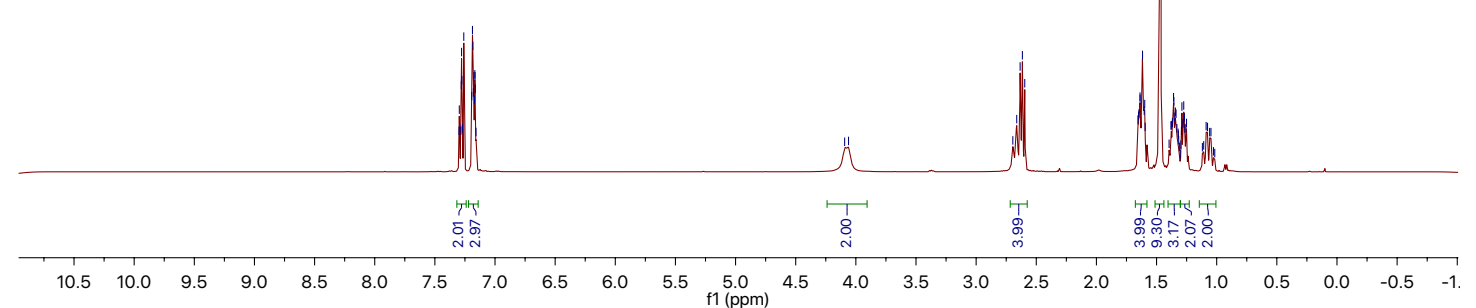

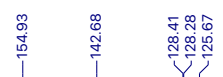

$\sqrt{2}$

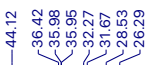

${ }^{13} \mathrm{C}$ NMR $\left(101 \mathrm{MHz}, \mathrm{CDCl}_{3}\right)$

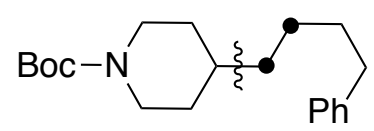

3a

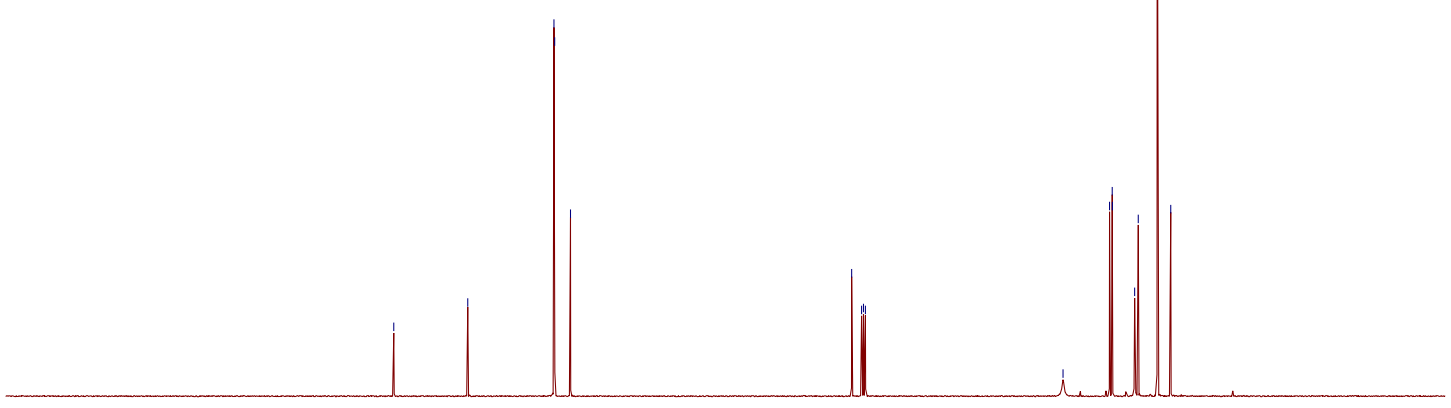

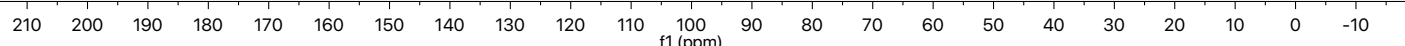


1H NMR (400 MHz, $\left.\mathrm{CDCl}_{3}\right)$
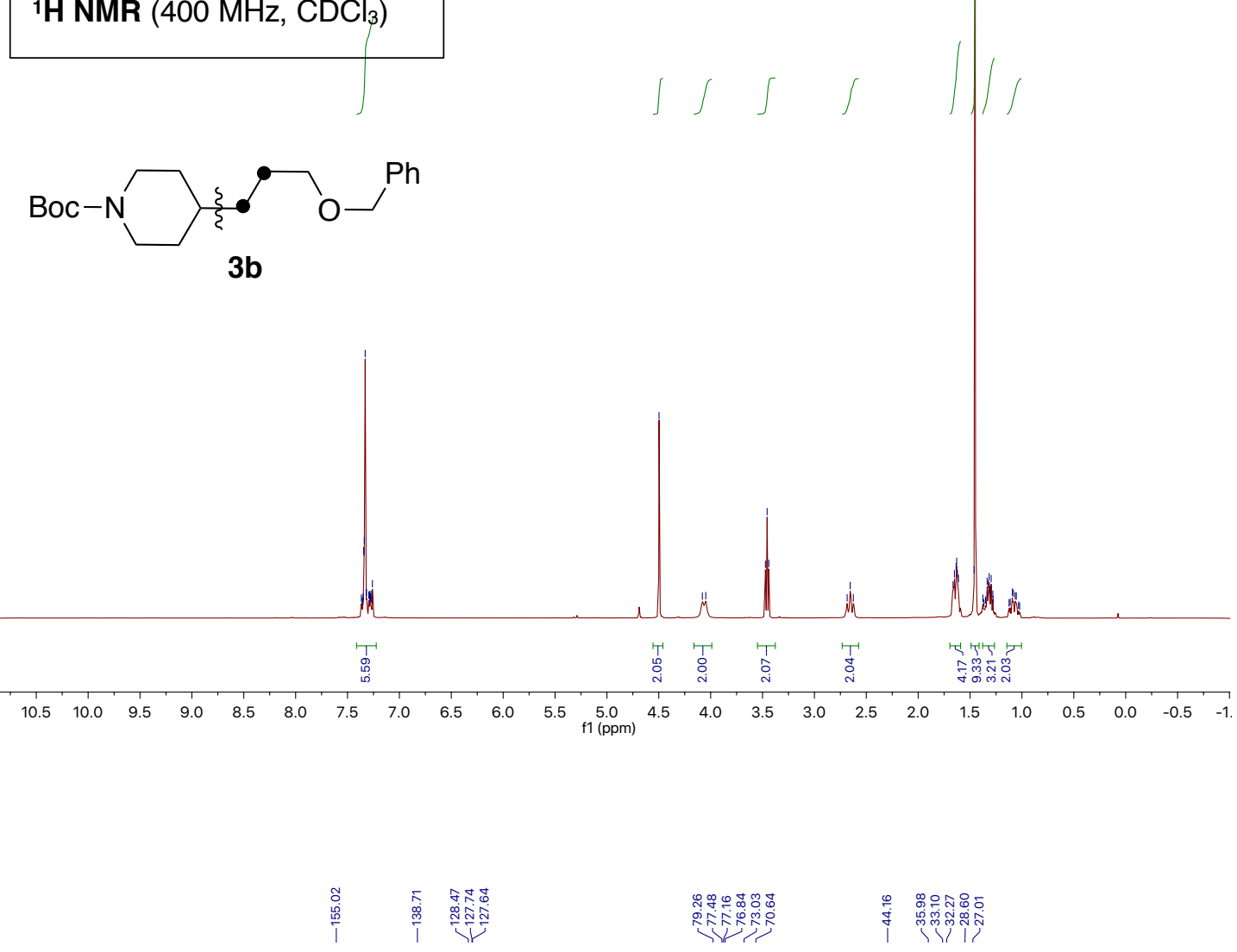

${ }^{13} \mathbf{C}$ NMR $\left(101 \mathrm{MHz}, \mathrm{CDCl}_{3}\right)$

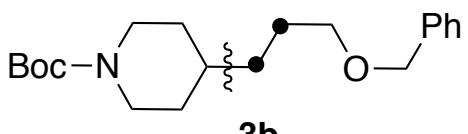

$3 b$

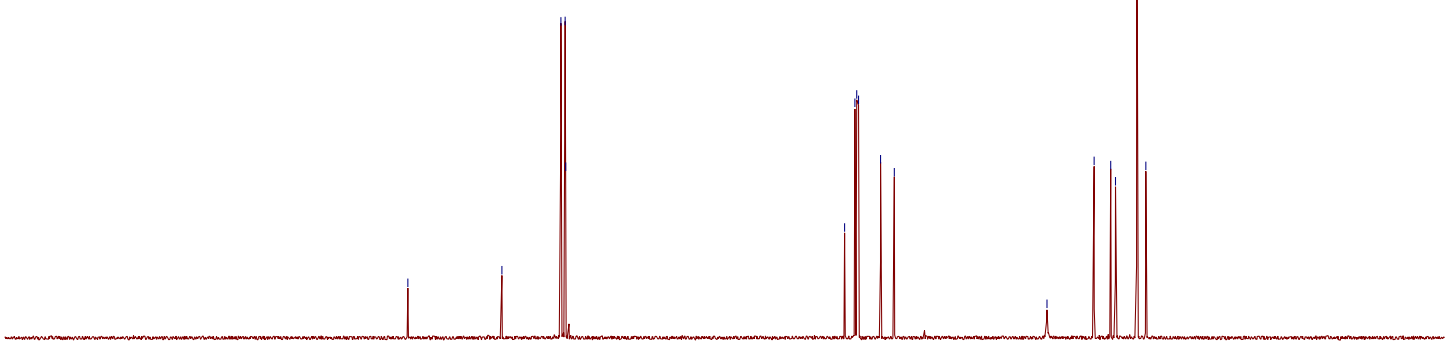

\begin{tabular}{lllllllllllllllllllllllllllllllllllll}
\hline & 220 & 210 & 200 & 190 & 180 & 170 & 160 & 150 & 140 & 130 & 120 & 110 & 100 & 90 & 80 & 70 & 60 & 50 & 40 & 30 & 20 & 10 & 0 & -10 & -20
\end{tabular} 

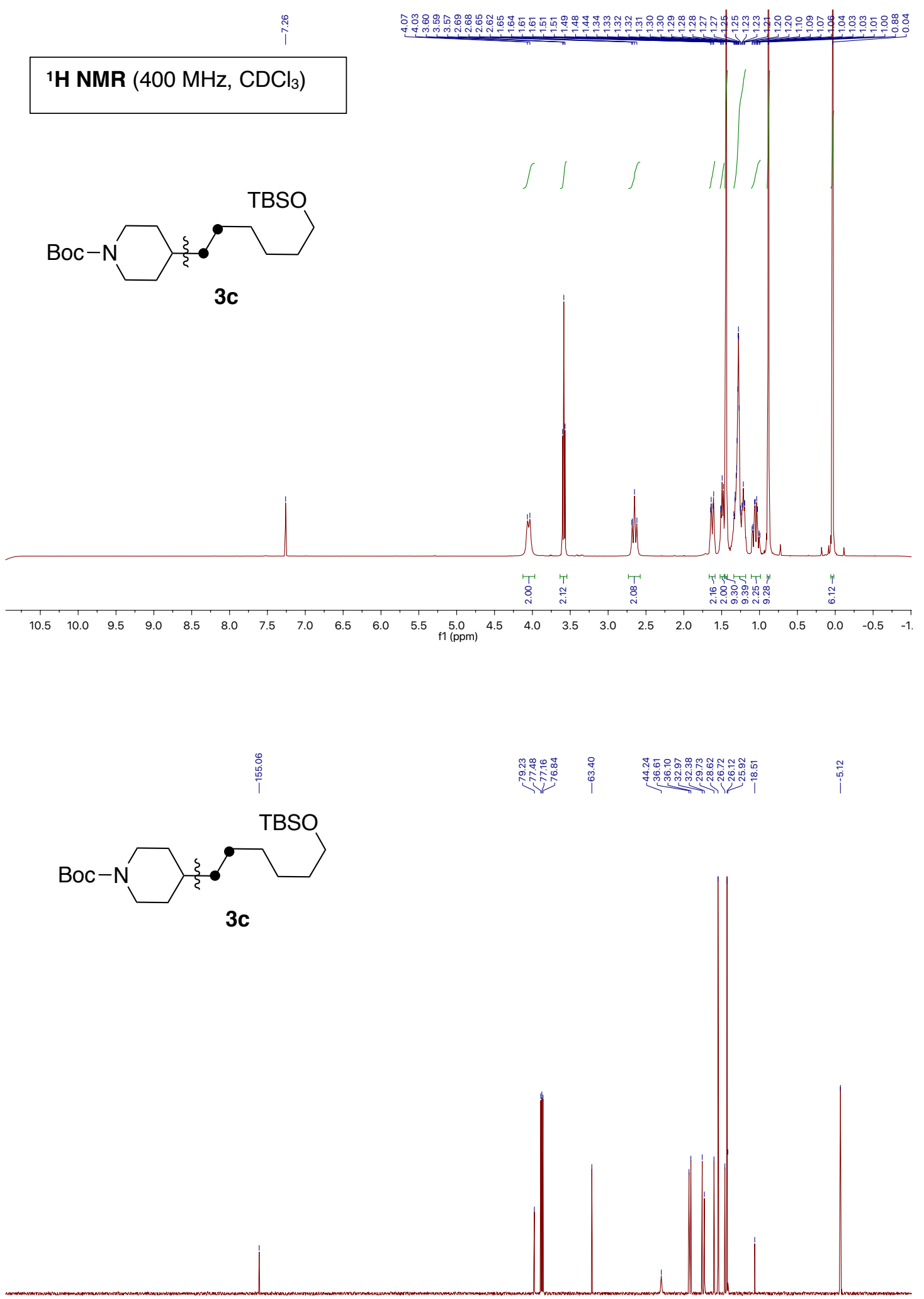

\begin{tabular}{llllllllllllllllllllllllllll}
\hline 220 & 210 & 200 & 190 & 180 & 170 & 160 & 150 & 140 & 130 & 120 & 110 & 100 & 100 & 80 & 70 & 60 & 50 & 40 & 30 & 20 & 10 & 0 & -10 & -20
\end{tabular} 
1H NMR (400 MHz, $\left.\mathrm{CDCl}_{3}\right)$<smiles>CC(C)(C)OC(=O)N1CCC(CCCCC#N)CC1</smiles>

3d
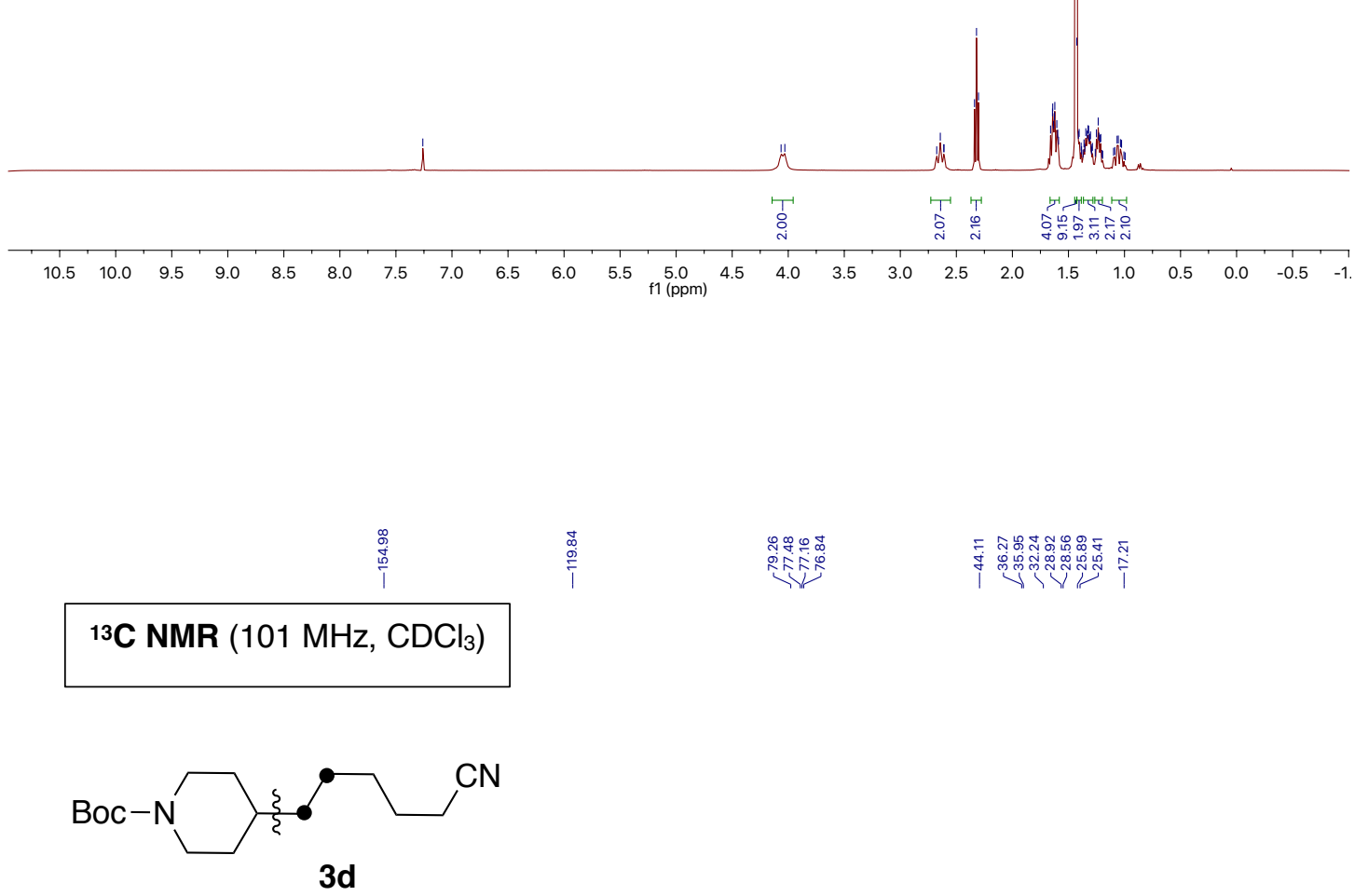

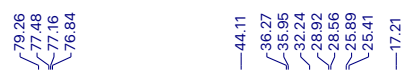

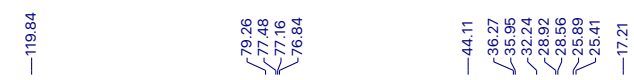

${ }^{13} \mathrm{C}$ NMR $\left(101 \mathrm{MHz}, \mathrm{CDCl}_{3}\right)$

$3 d$

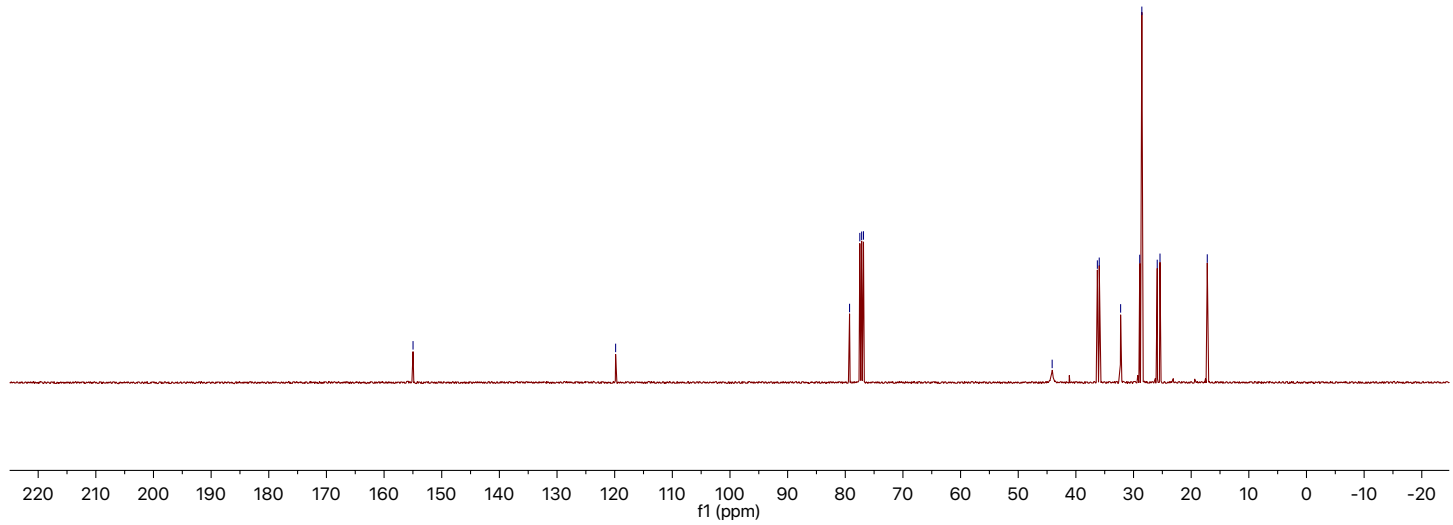



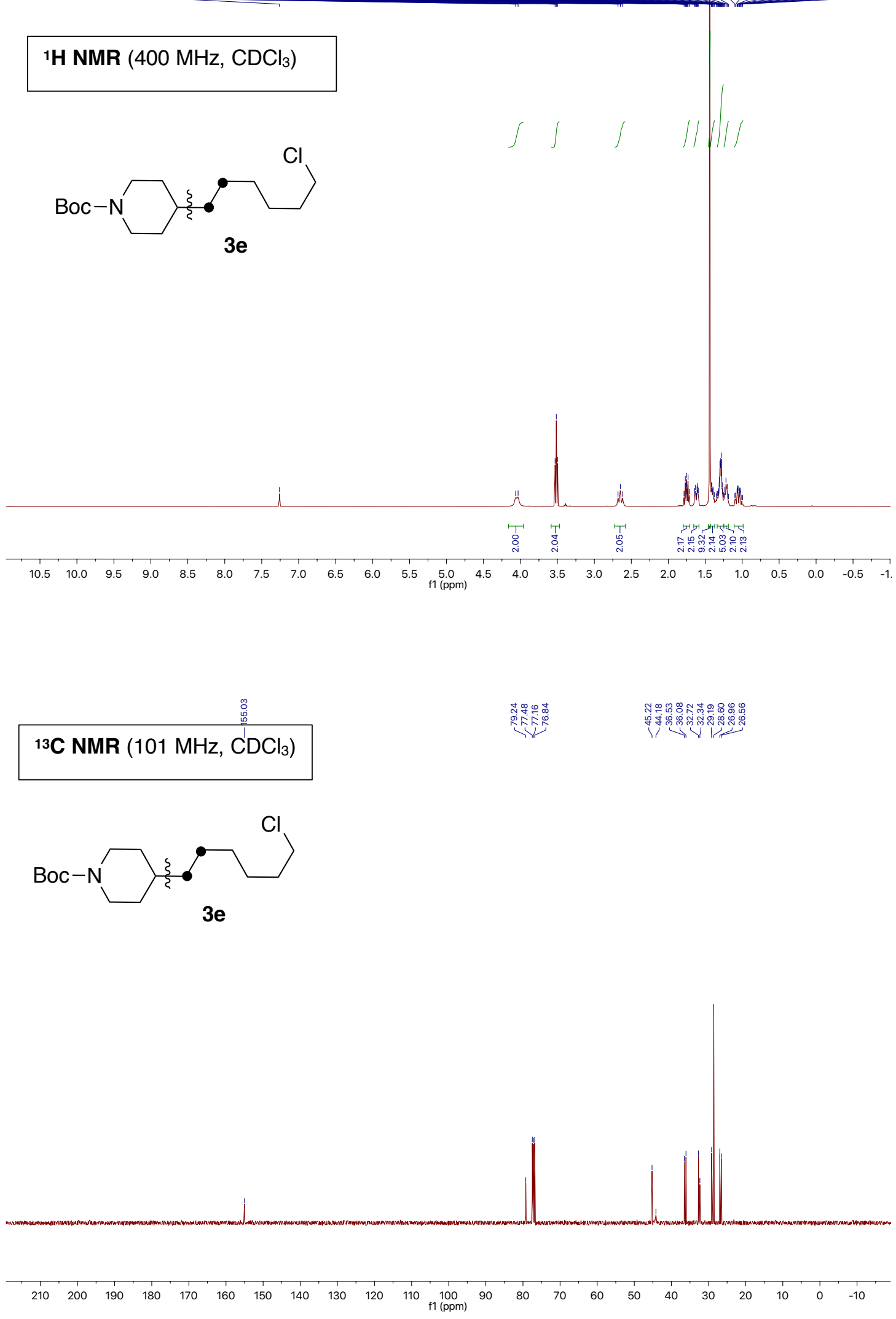
'1H NMR $\left(400 \mathrm{MHz}, \mathrm{CDCl}_{3}\right)$<smiles>CC(C)(C)OC(=O)N1CCC(CCCCCBr)CC1</smiles>
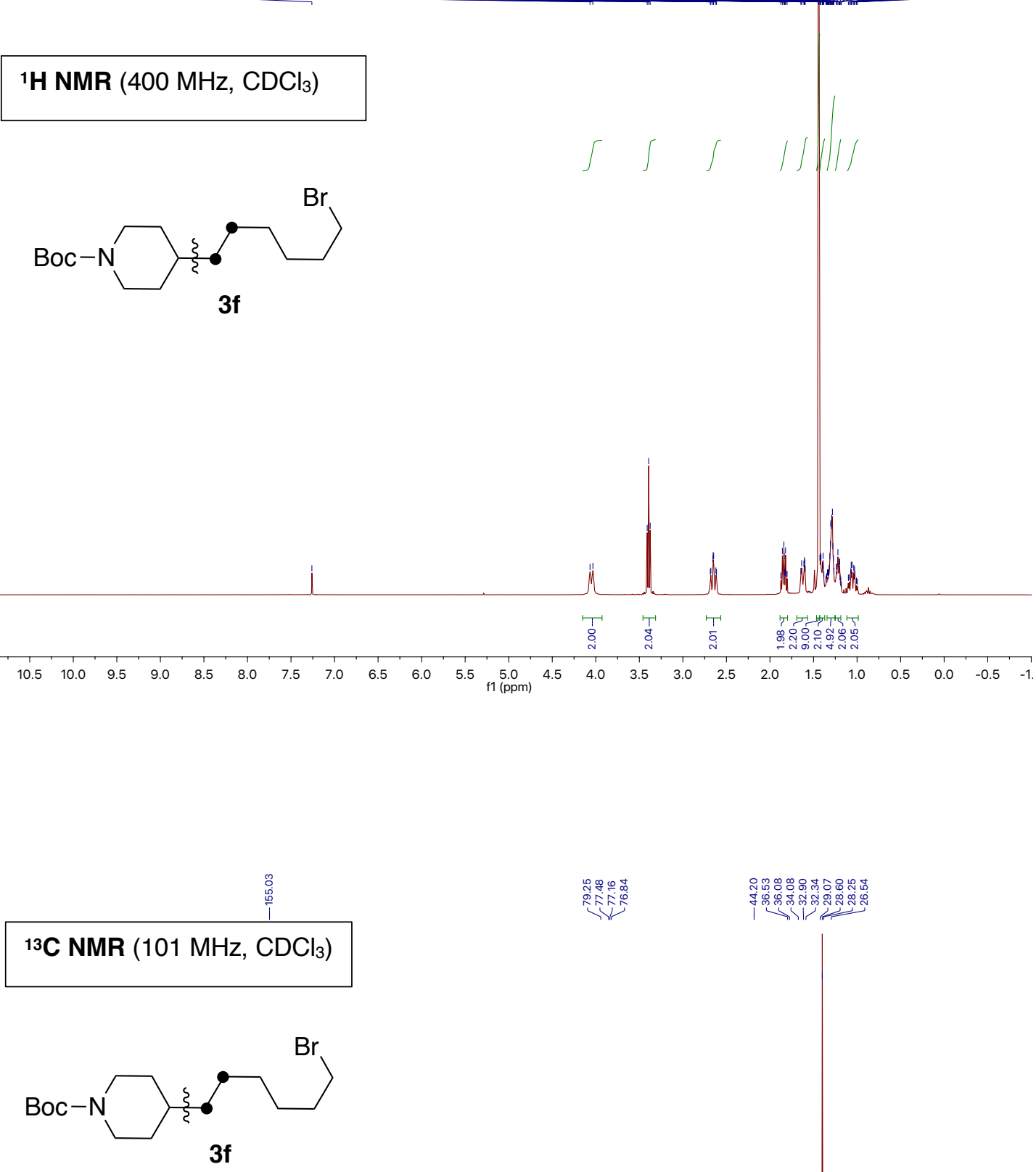

${ }^{13} \mathrm{C} \mathrm{NMR}\left(101 \mathrm{MHz}, \mathrm{CDCl}_{3}\right)$

$\mathrm{Br}$

$3 f$
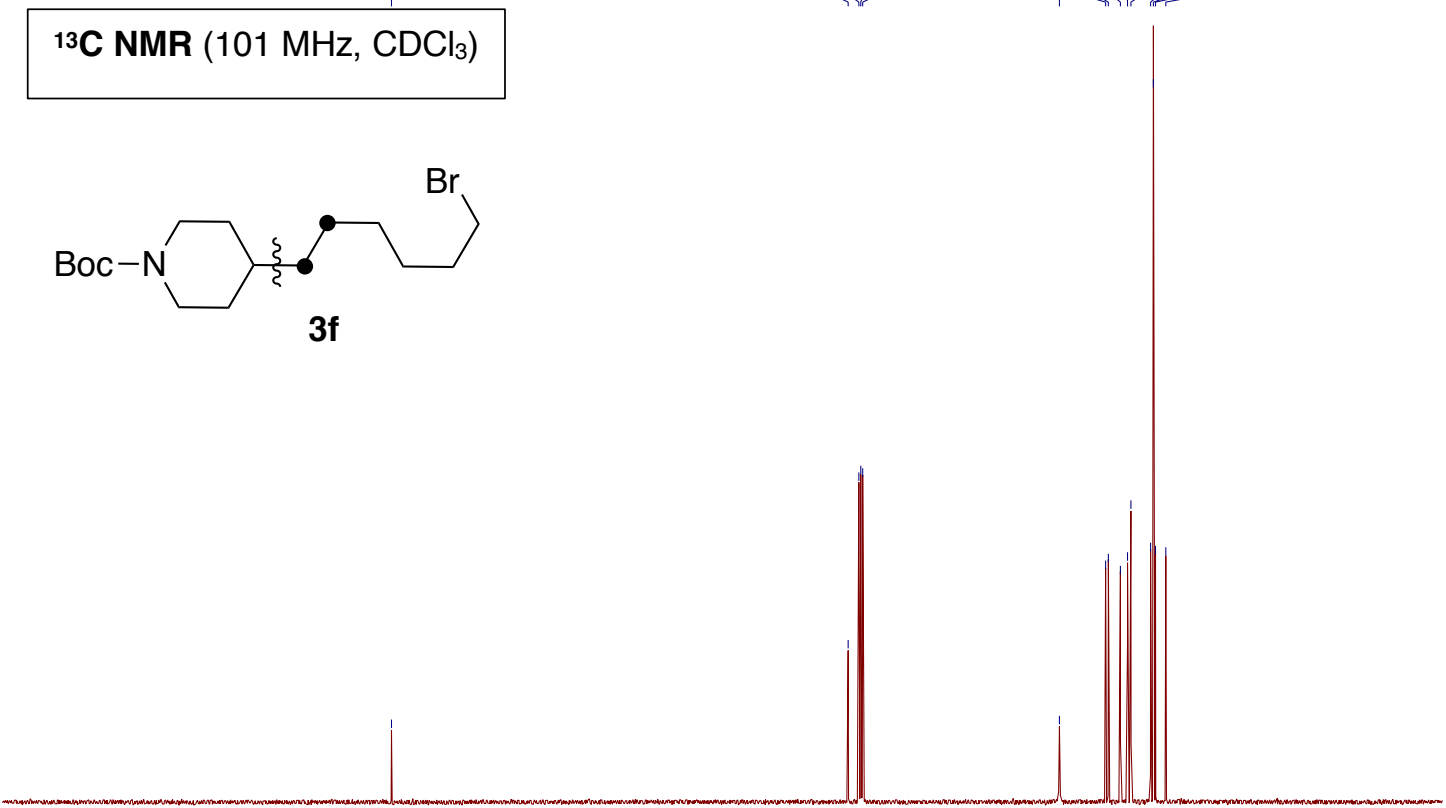

$\begin{array}{lllllllllllllllllllllllllll}210 & 200 & 190 & 180 & 170 & 160 & 150 & 140 & 130 & 120 & 110 & 100 & 90 & 80 & 70 & 60 & 50 & 40 & 30 & 20 & 10 & 0 & -10\end{array}$ 

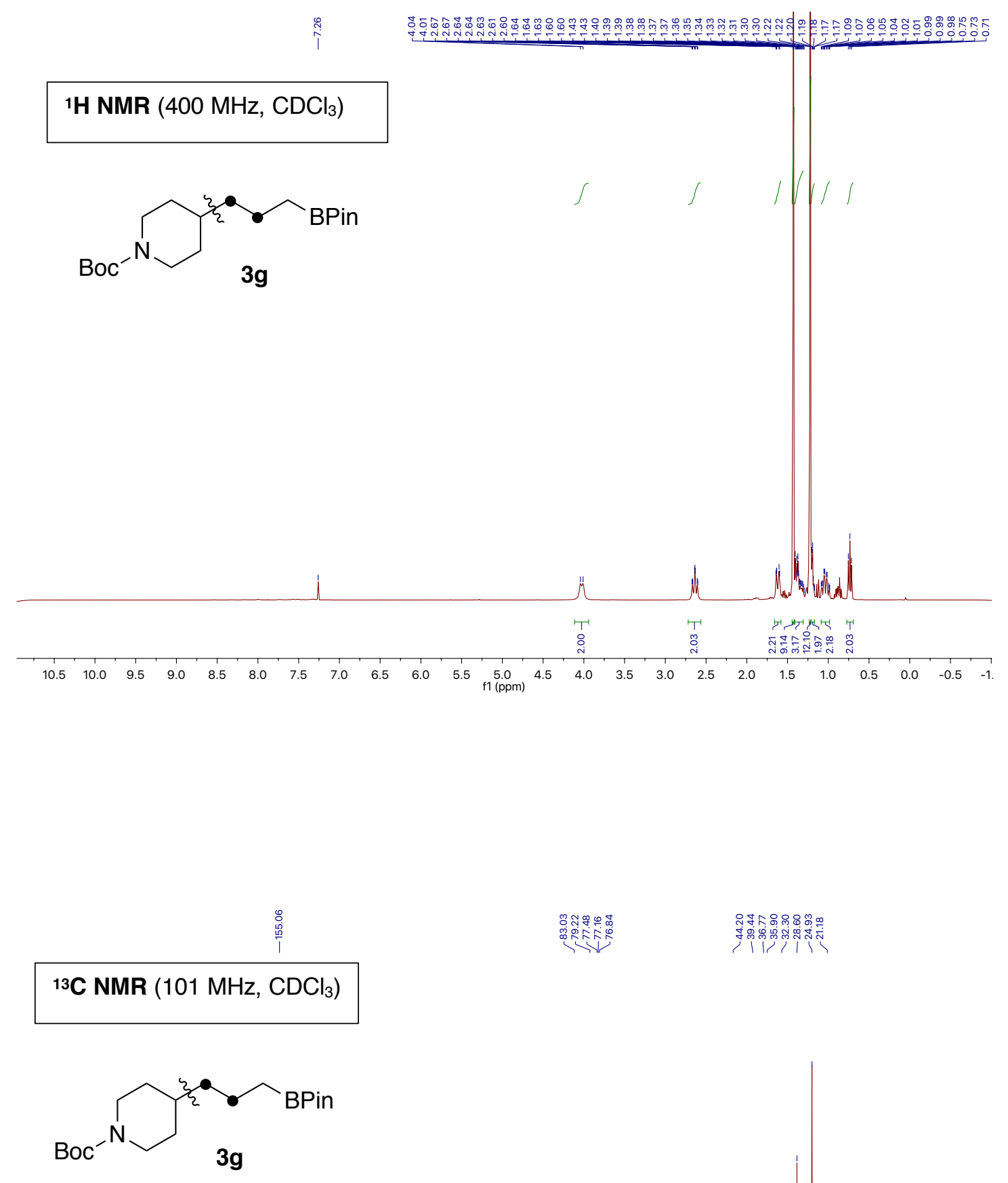

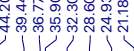

${ }^{13} \mathrm{C}$ NMR $\left(101 \mathrm{MHz}, \mathrm{CDCl}_{3}\right)$

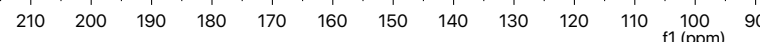


11B NMR (128 MHz, $\left.\mathrm{CDCl}_{3}\right)$

$\mathrm{Boc}^{-\mathrm{N}}$ BPin

\begin{tabular}{llllllllllllllllllllllllllllll}
\hline 120 & 110 & 100 & 90 & 80 & 70 & 60 & 50 & 40 & 30 & 20 & 10 & $\underset{f 1}{(\mathrm{ppm})}$ & -10 & -20 & -30 & -40 & -50 & -60 & -70 & -80 & -90 & -100 & -110 & -120
\end{tabular}

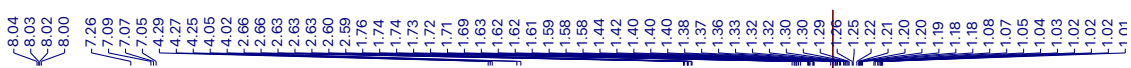

1H NMR $\left(400 \mathrm{MHz}, \mathrm{CDCl}_{3}\right)$
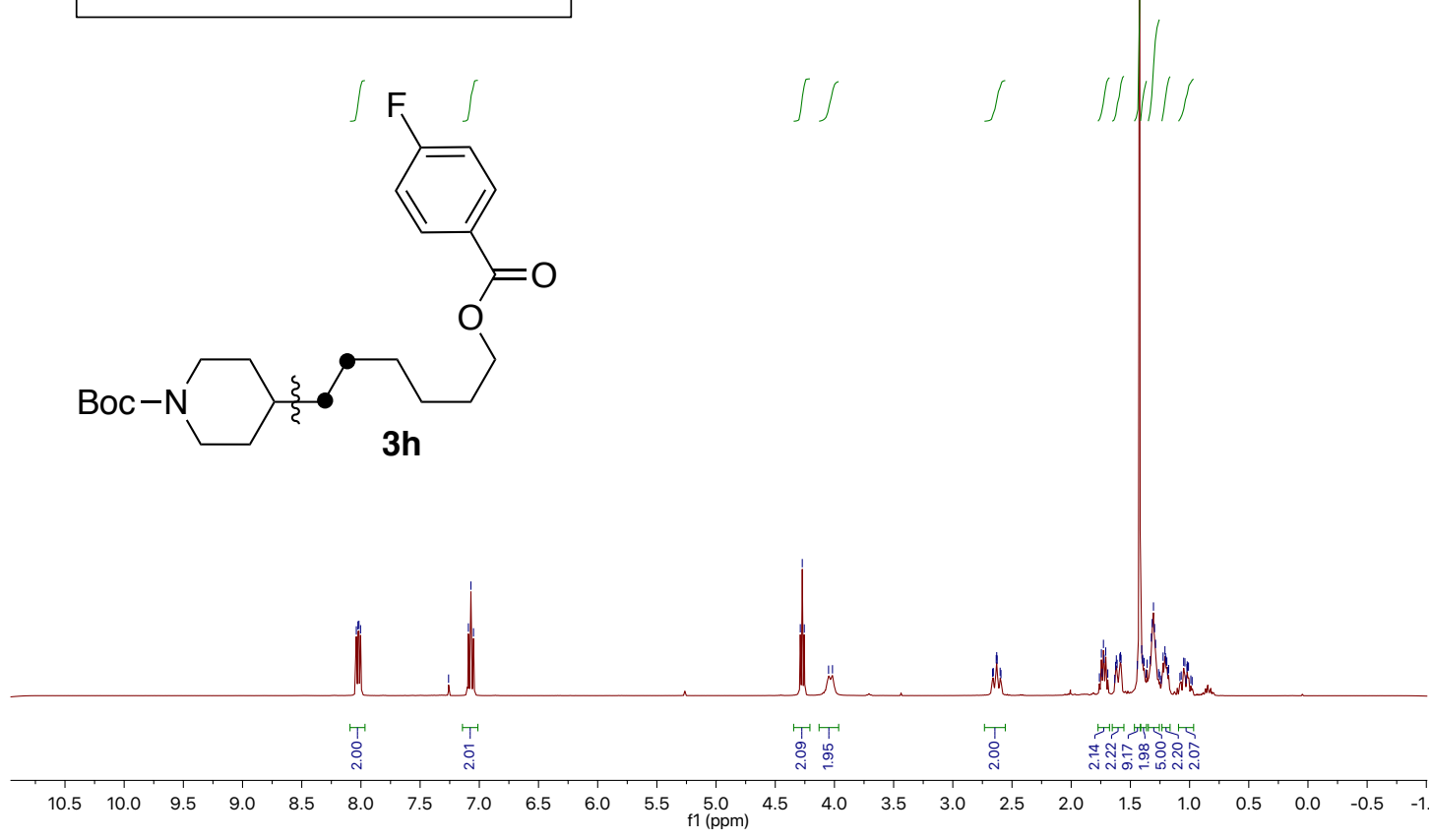


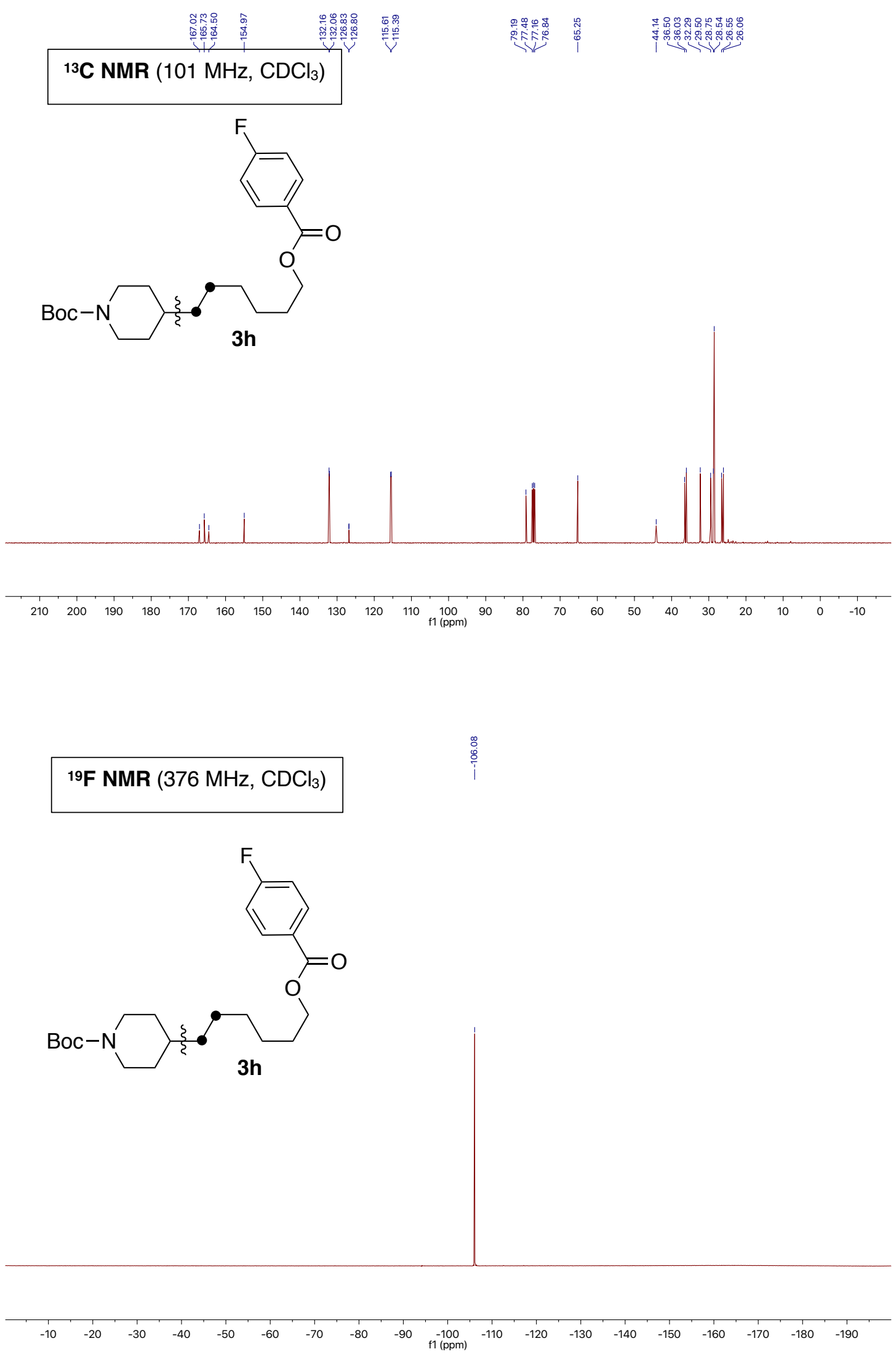




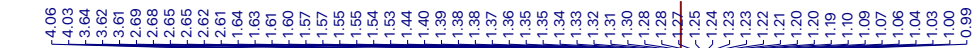

1H NMR (400 MHz, $\left.\mathrm{CDCl}_{3}\right)$
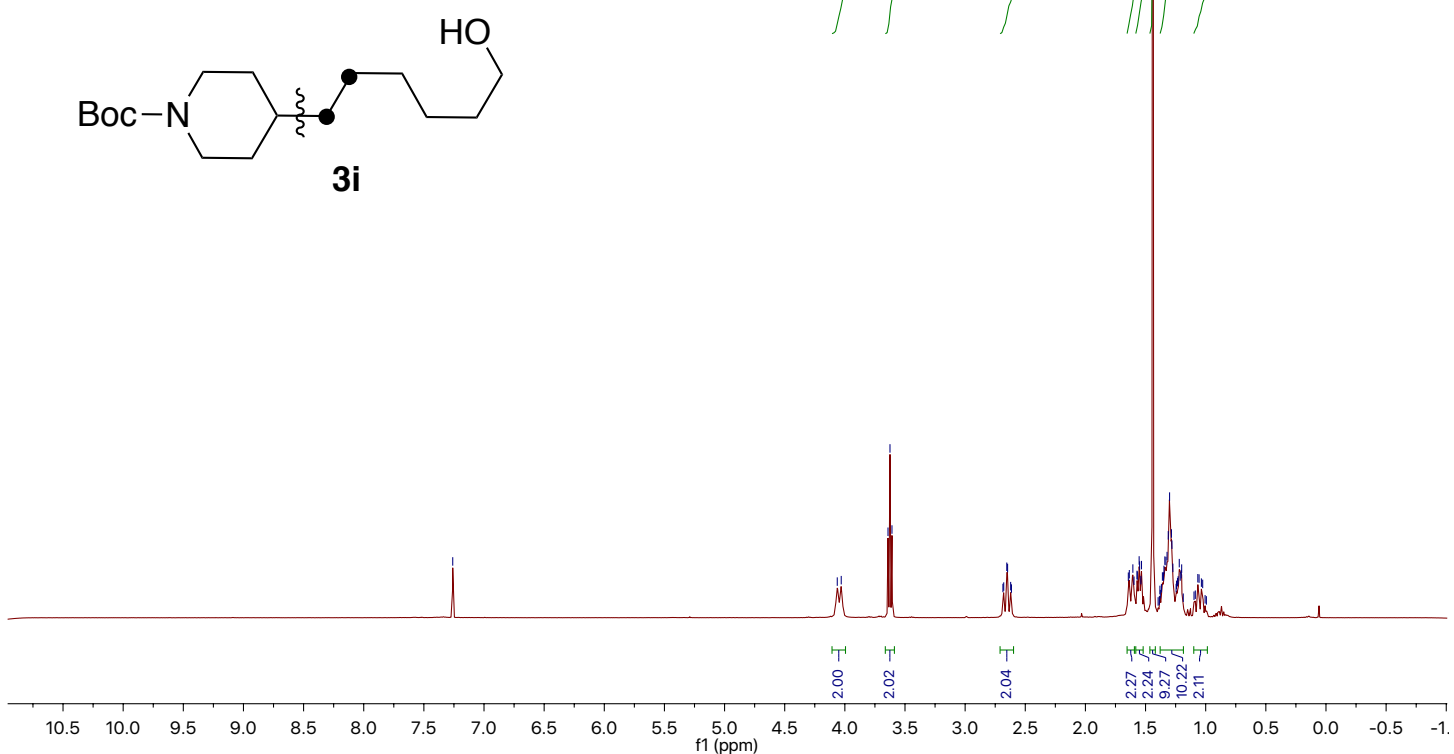

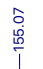

${ }^{13} \mathrm{C}$ NMR $\left(101 \mathrm{MHz}, \mathrm{CDCl}_{3}\right)$

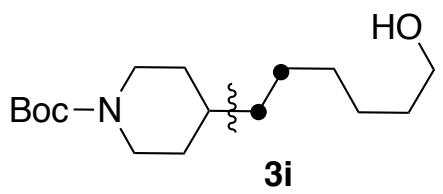

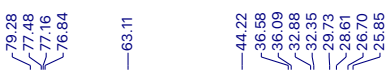

$3 \mathbf{i}$

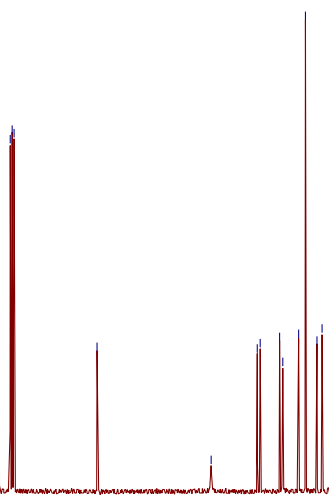

$\begin{array}{llllllllllll}210 & 200 & 190 & 180 & 170 & 160 & 150 & 140 & 130 & 120 & 110 \begin{array}{c}100 \\ \mathrm{f} 1(\mathrm{ppm})\end{array} & 90\end{array}$ 
'H NMR $\left(400 \mathrm{MHz}, \mathrm{CDCl}_{3}\right)$<smiles>CC(=O)CCCCC1CCCCC1</smiles>

3j

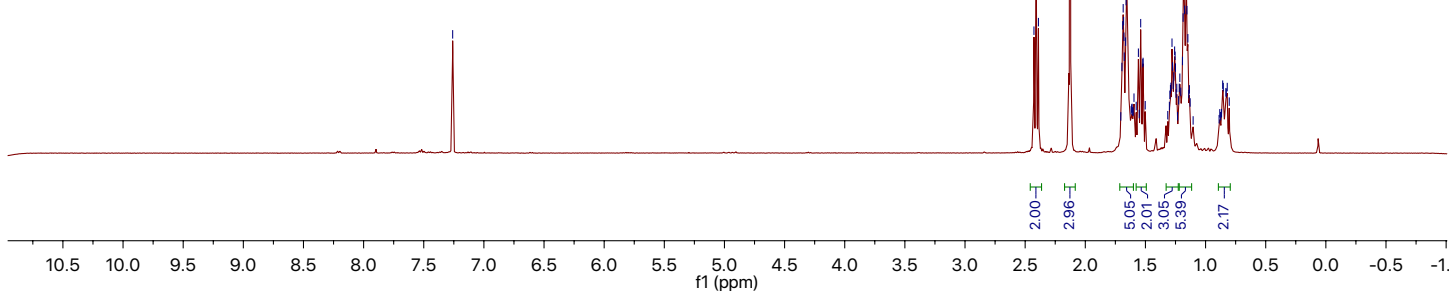

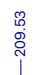

${ }^{13} \mathrm{C} \mathrm{NMR}\left(101 \mathrm{MHz}, \mathrm{CDCl}_{3}\right)$<smiles>CC(=O)CCCCC1CCCCC1</smiles>

3j

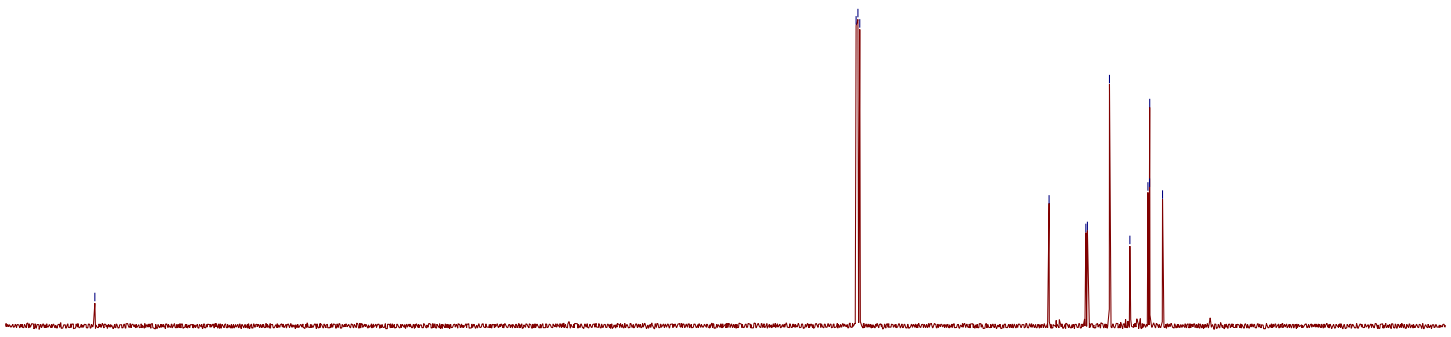

\begin{tabular}{lllllllllllllllllllllllllllllllll}
\hline & 220 & 210 & 200 & 190 & 180 & 170 & 160 & 150 & 140 & 130 & 120 & 110 & 100 & 90 & 80 & 70 & 60 & 50 & 40 & 30 & 20 & 10 & 0 & -10 & -20
\end{tabular} 
1H NMR (400 MHz, $\left.\mathrm{CDCl}_{3}\right)$
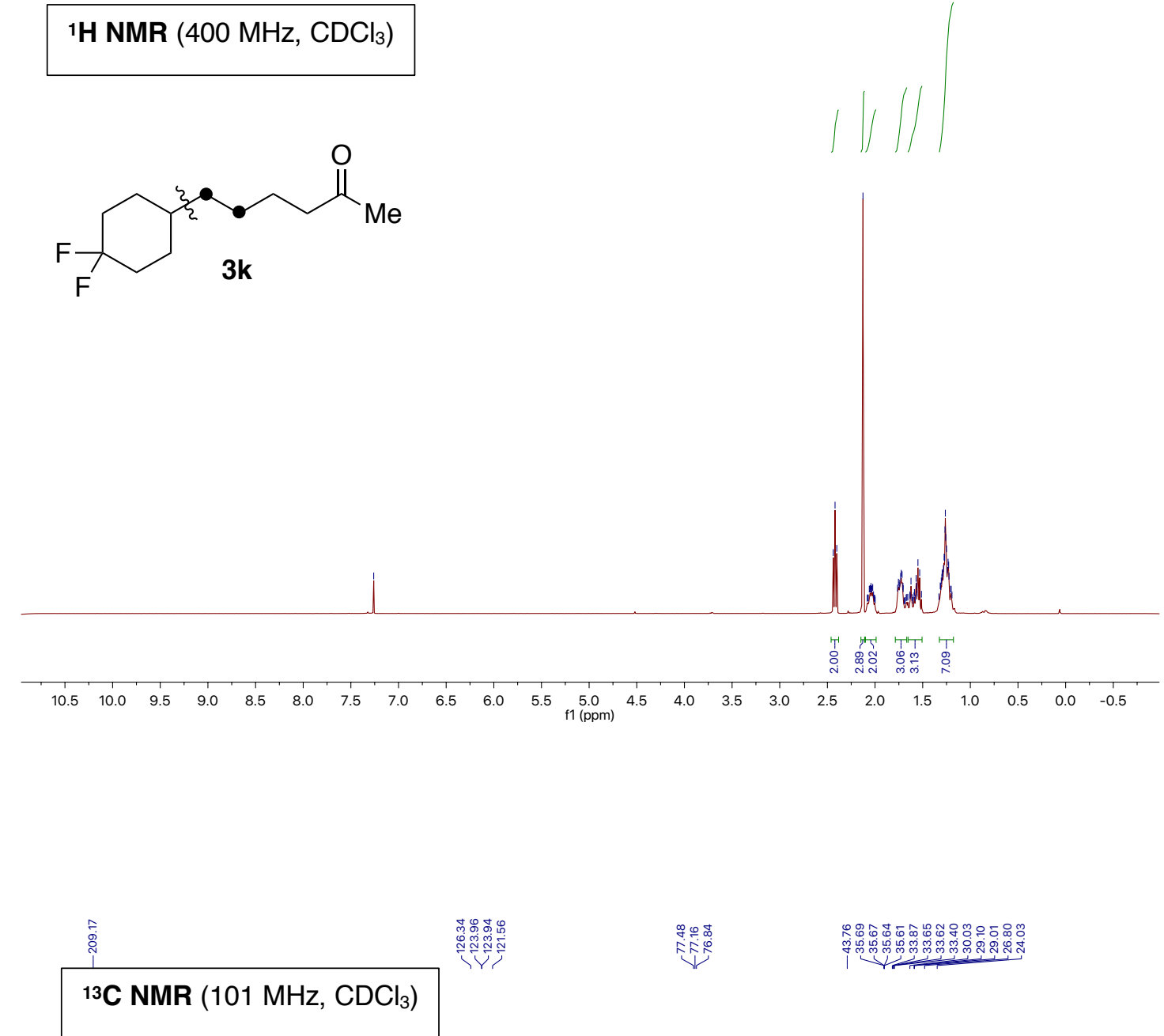<smiles>CC(=O)CCCCCC1CCC(F)(F)CC1</smiles>

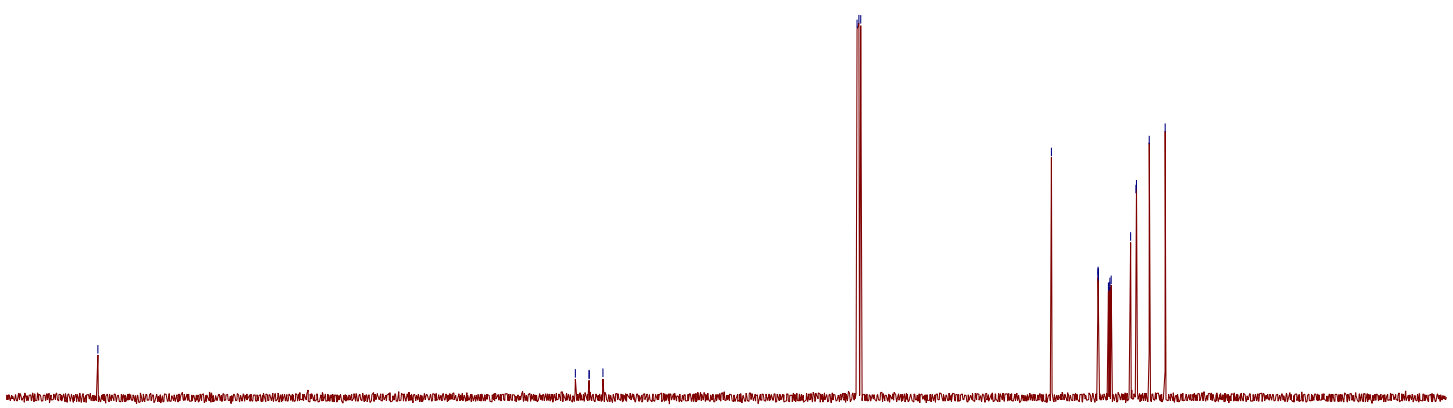

\begin{tabular}{lllllllllllllllllllllllllllllllllll}
\hline & 220 & 210 & 200 & 190 & 180 & 170 & 160 & 150 & 140 & 130 & 120 & 110 & 100 & 90 & 80 & 70 & 60 & 50 & 40 & 30 & 20 & 10 & 0 & -10 & -20
\end{tabular} 
19F NMR (376 MHz, $\left.\mathrm{CDCl}_{3}\right)$<smiles>CC(=O)CCCCC1CCC(F)(F)CC1</smiles>
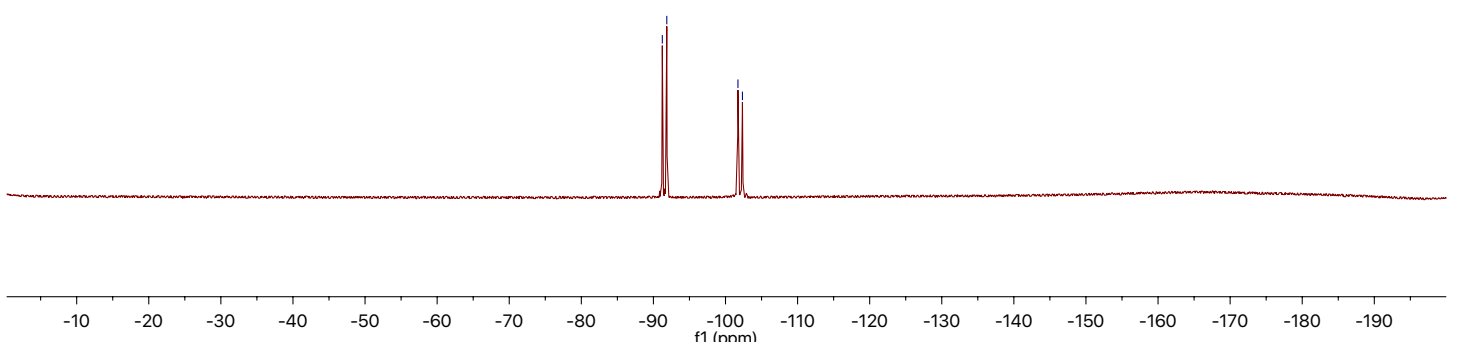

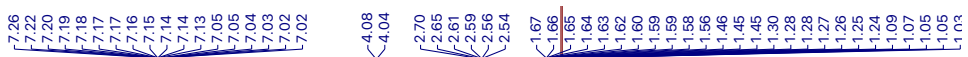

'H NMR (400 MHz, $\left.\mathrm{CDCl}_{3}\right)$<smiles>CC(C)(C)OC(=O)N1CCC(CCCc2cccc(Cl)c2)CC1</smiles><smiles></smiles>

Boc

3I

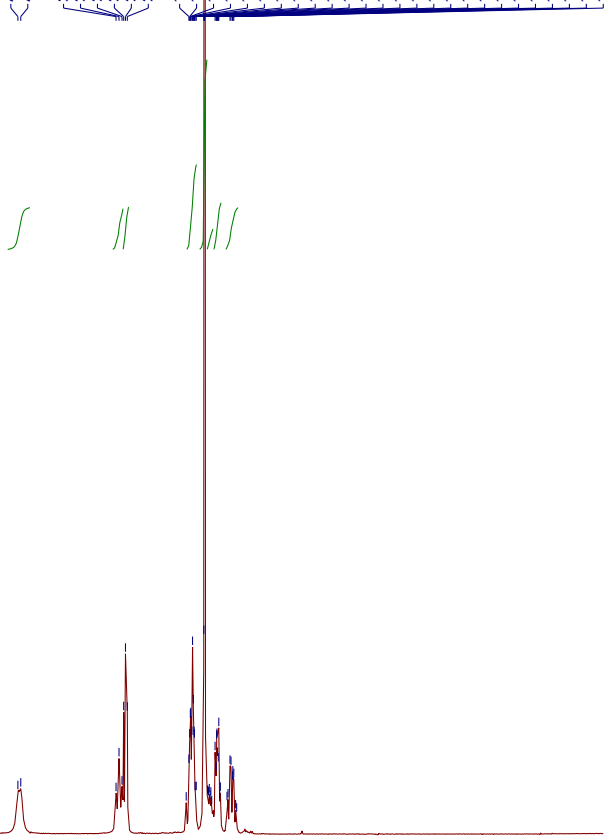

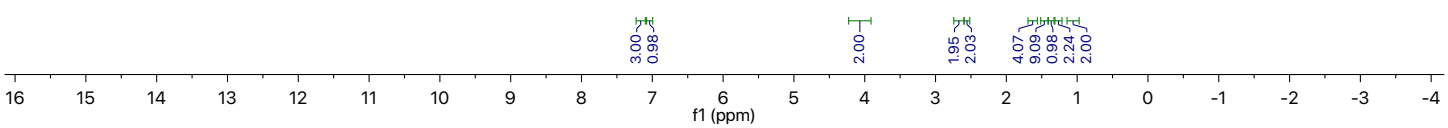




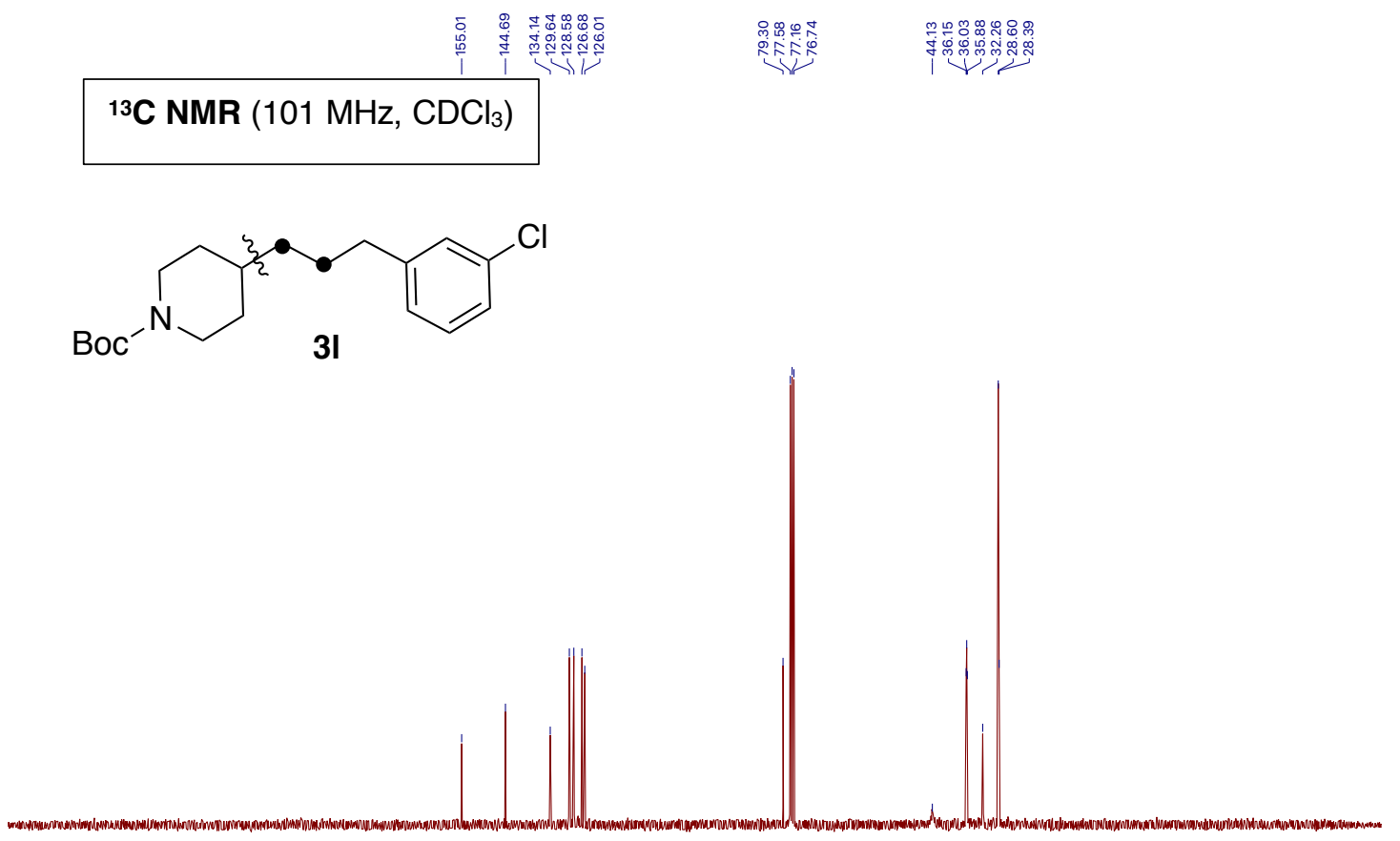

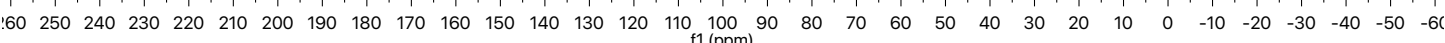

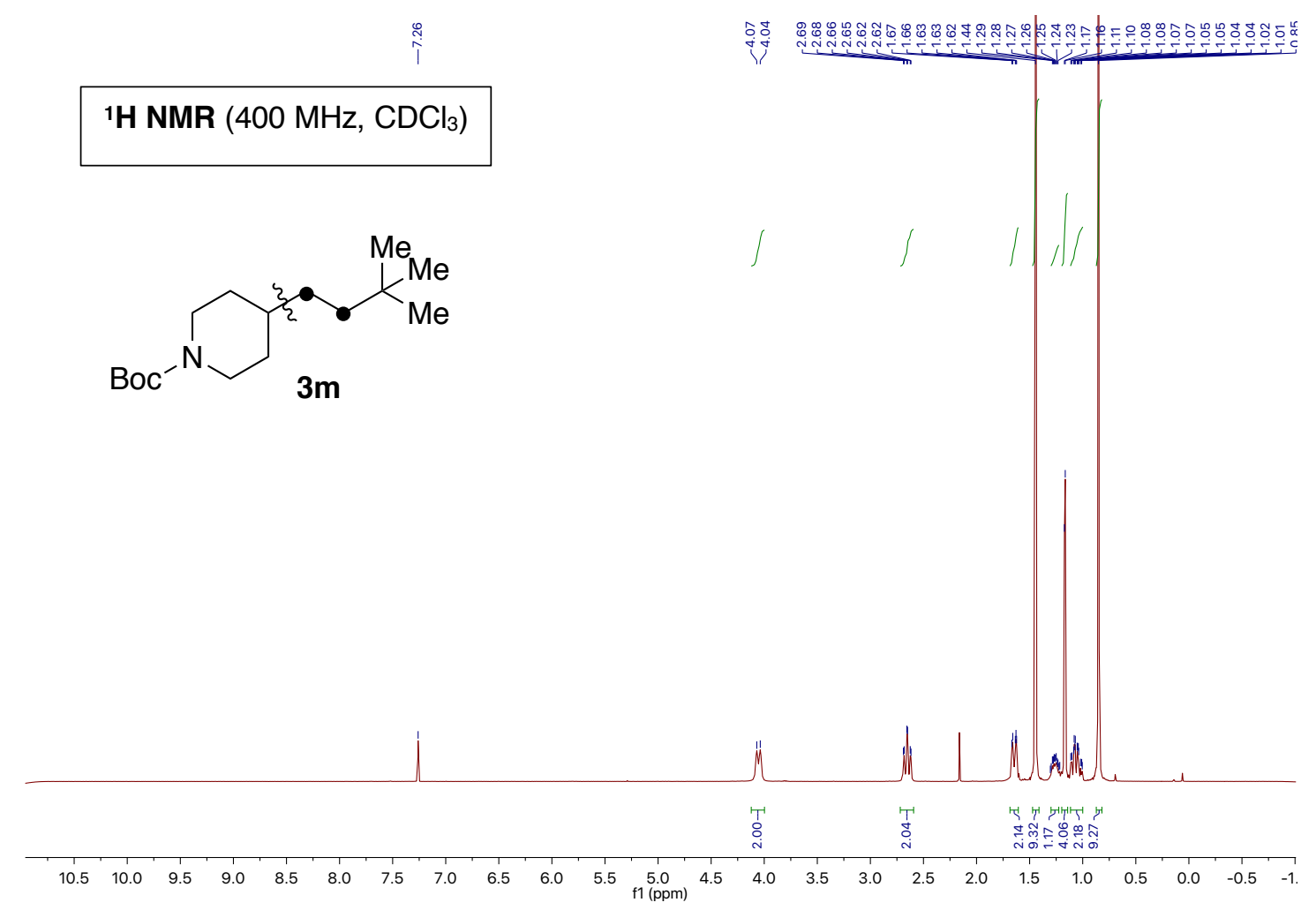




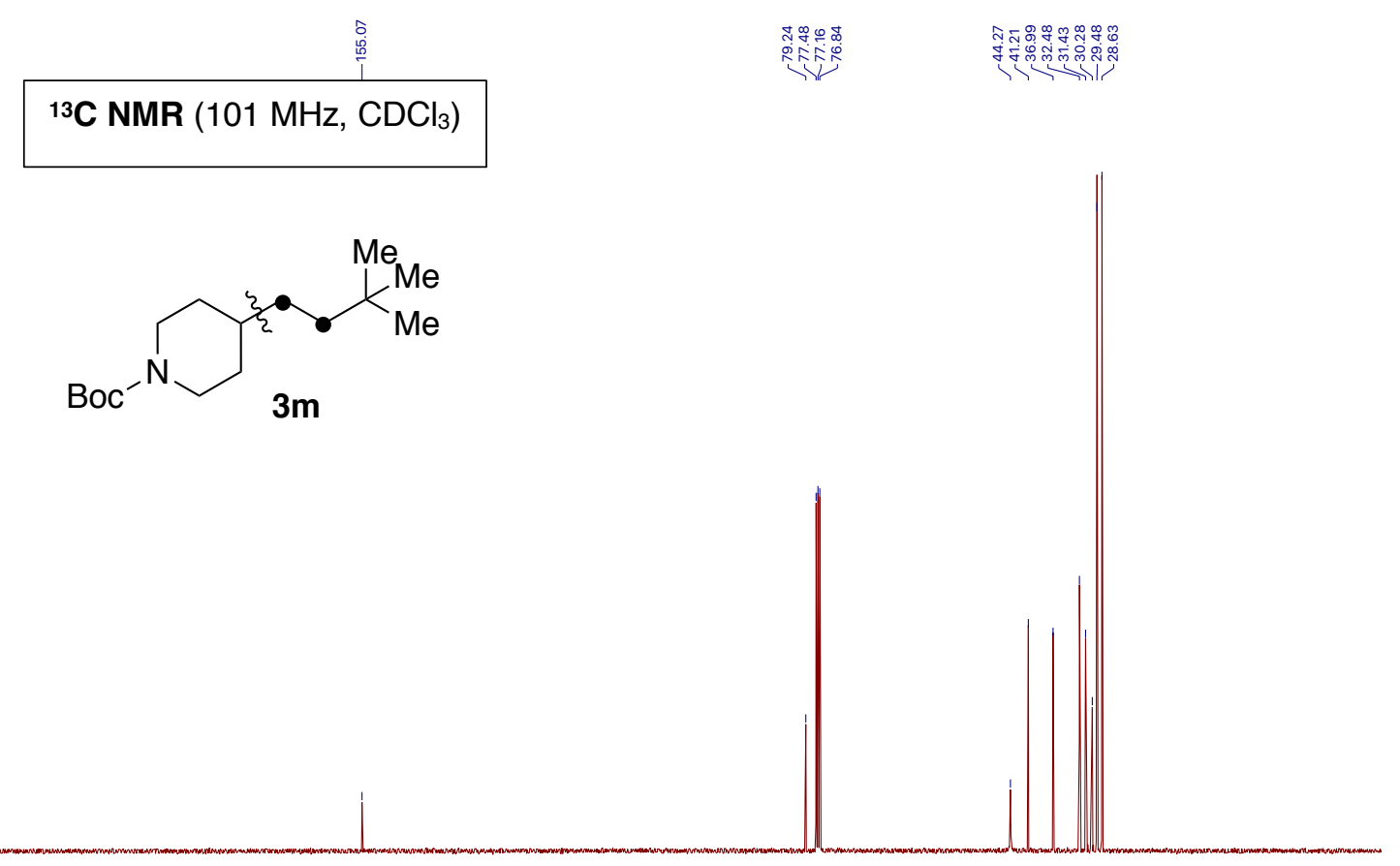

$\begin{array}{lllllllllllllllllllllllllllllllllll}210 & 200 & 190 & 180 & 170 & 160 & 150 & 140 & 130 & 120 & 110 & 100 & 90 & 80 & 70 & 60 & 50 & 40 & 30 & 20 & 10 & 0 & -10\end{array}$

7.

1H NMR (400 MHz, $\left.\mathrm{CDCl}_{3}\right)$
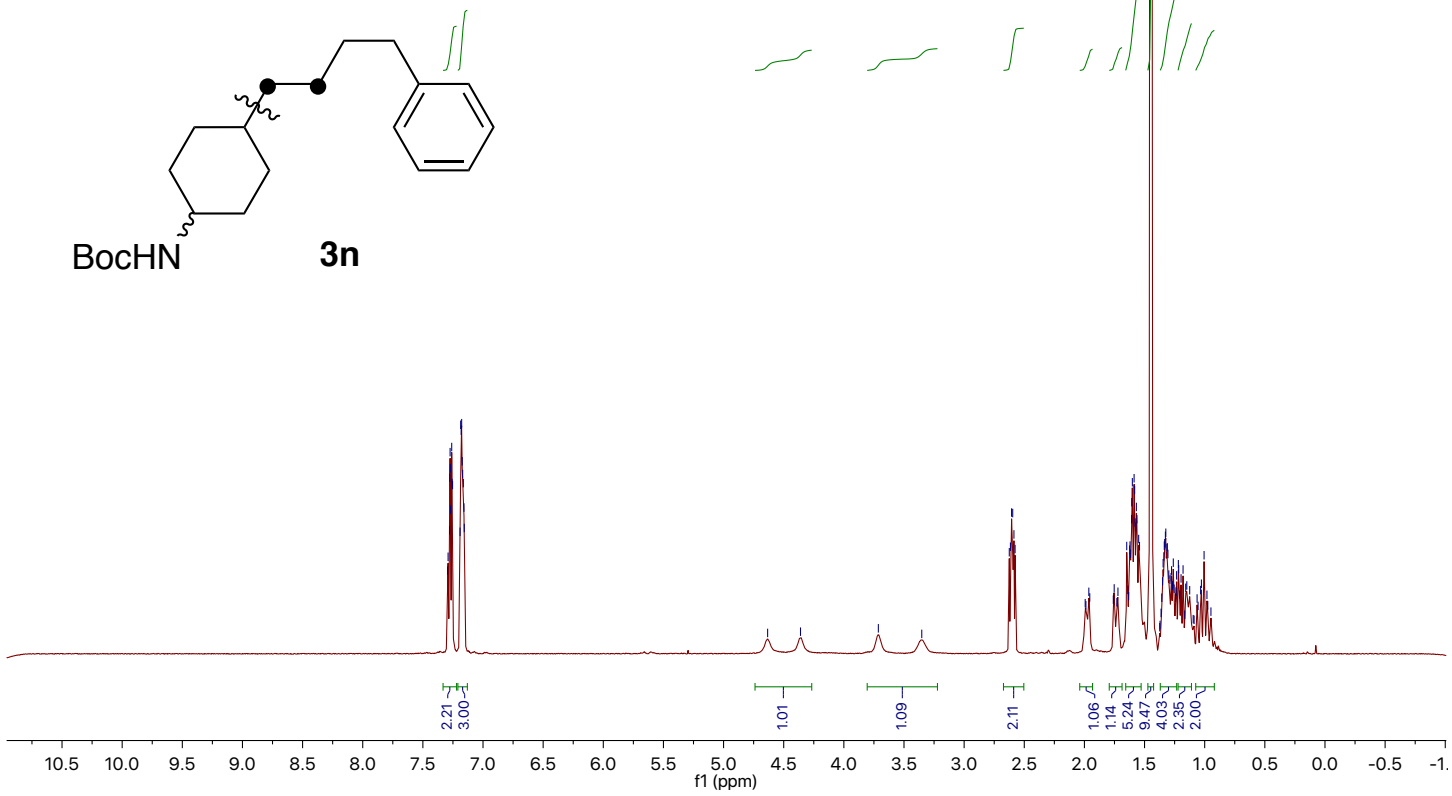


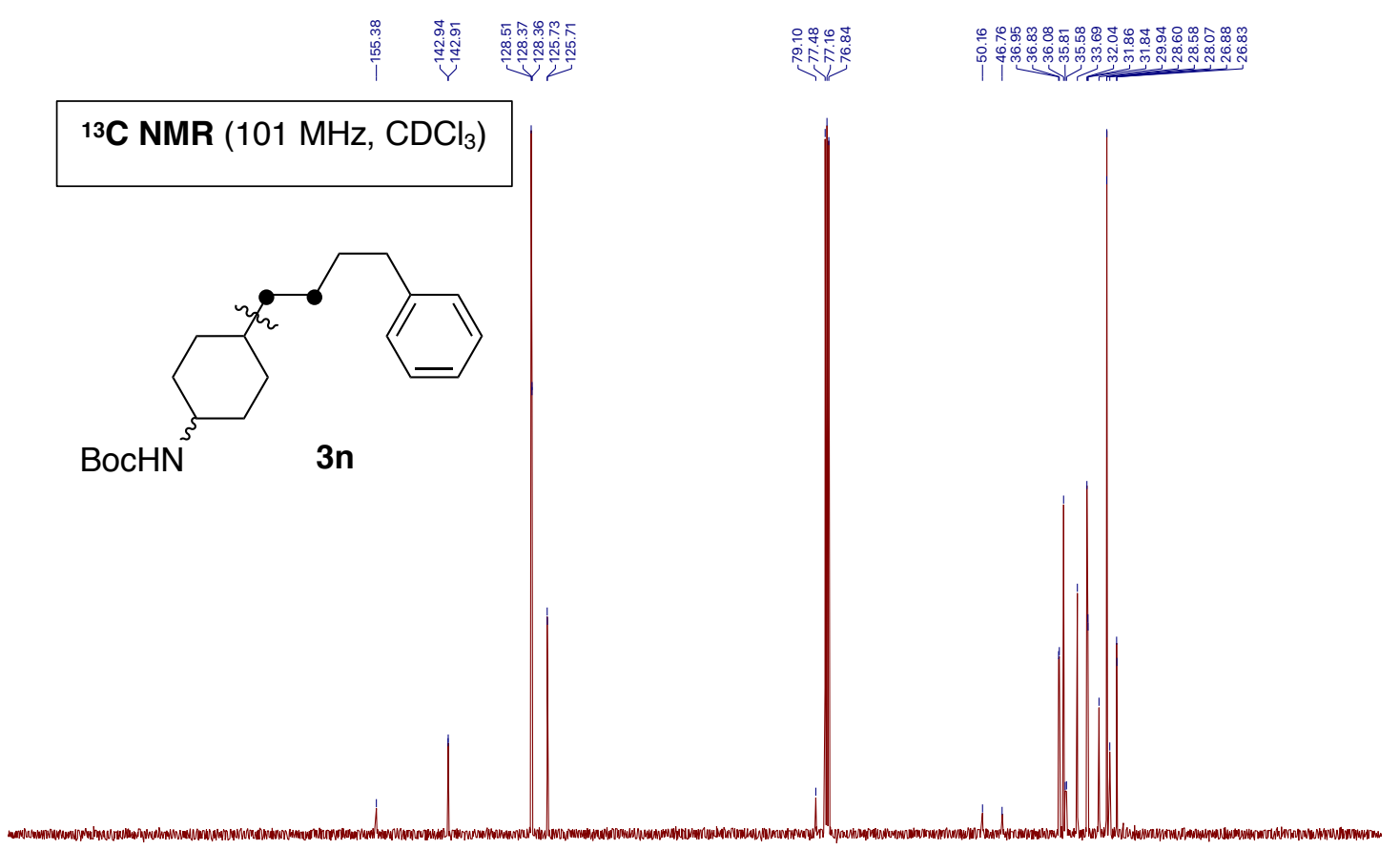

$\begin{array}{lllllllllllllllllllllll}210 & 200 & 190 & 180 & 170 & 160 & 150 & 140 & 130 & 120 & 110 & 100 & 90 & 80 & 70 & 60 & 50 & 40 & 30 & 20 & 10 & 0 & -10\end{array}$

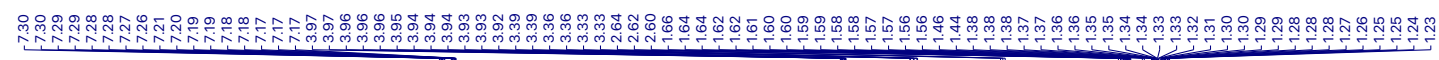

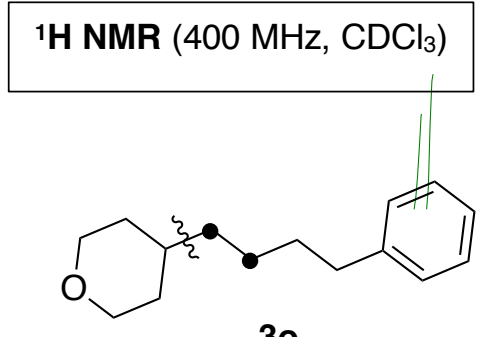

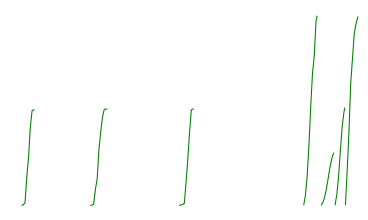

30

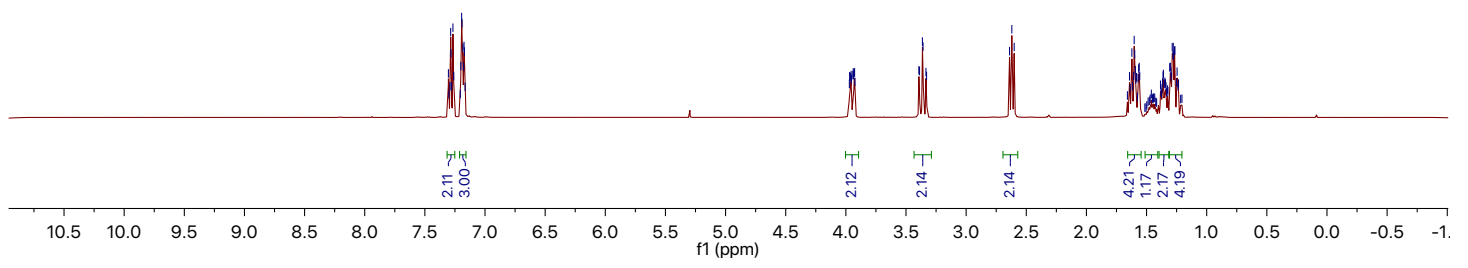




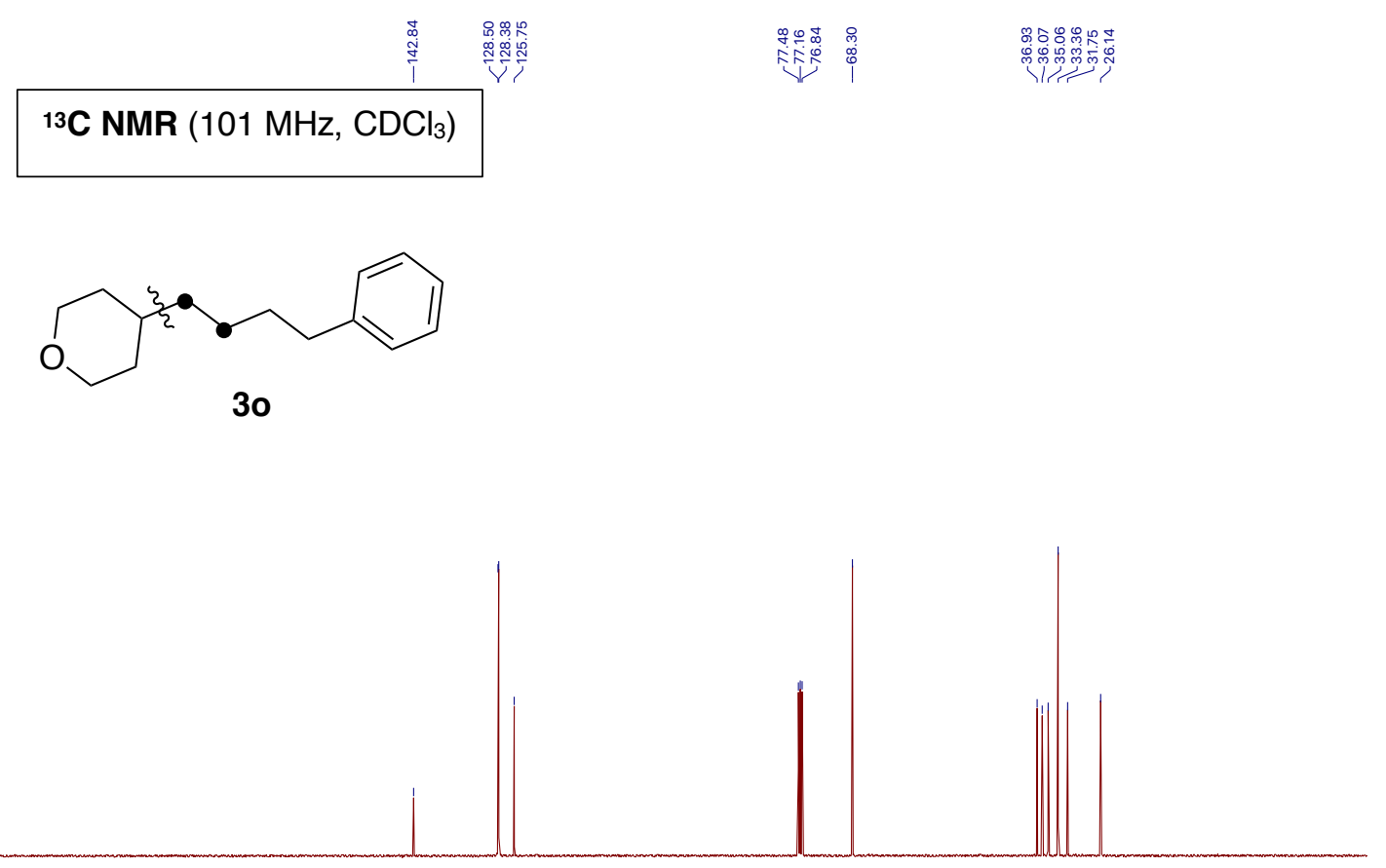

$\begin{array}{llllllllllllllllllllllll}210 & 200 & 190 & 180 & 170 & 160 & 150 & 140 & 130 & 120 & 110 & \begin{array}{c}100 \\ \mathrm{f}(\mathrm{ppm})\end{array} & 90 & 80 & 70 & 60 & 50 & 40 & 30 & 20 & 10 & 0 & -10\end{array}$

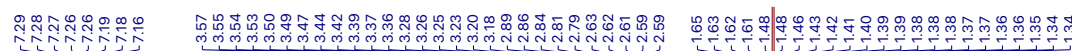

1H NMR $\left(400 \mathrm{MHz}, \mathrm{CDCl}_{3}\right)$

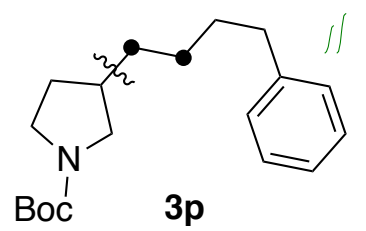

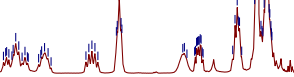

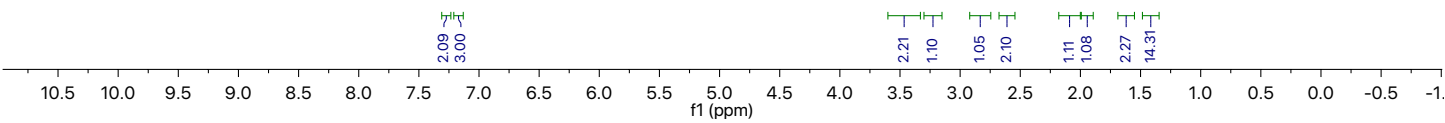


${ }^{13} \mathrm{C}$ NMR $\left(101 \mathrm{MHz}, \mathrm{CDCl}_{3}\right)$

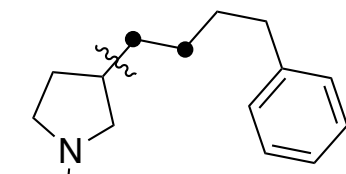

Boc 3p

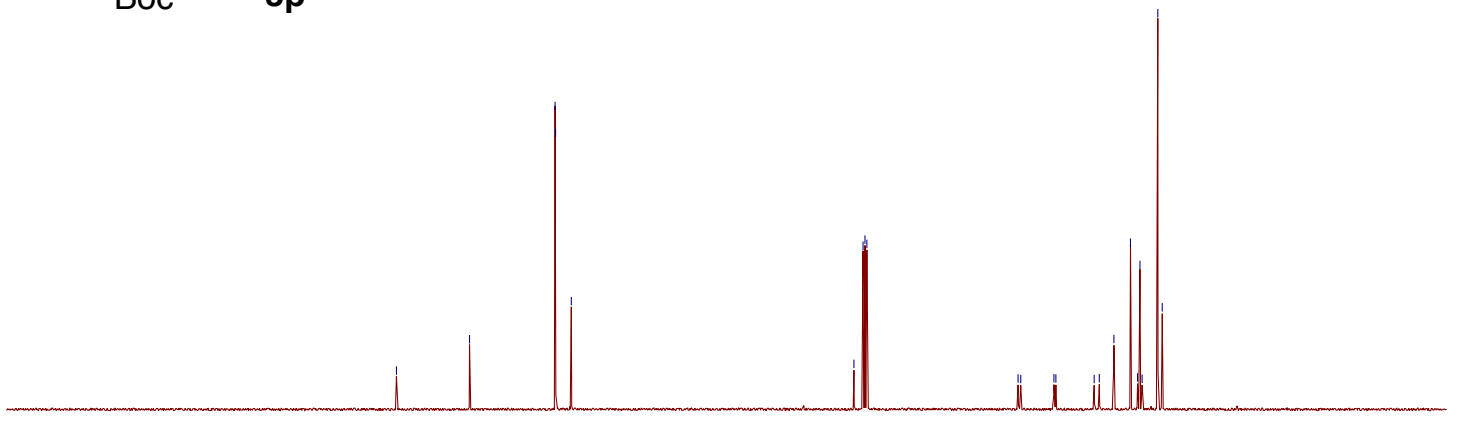

$\begin{array}{llllllllllllllllllllllllllllllllllllll}210 & 200 & 190 & 180 & 170 & 160 & 150 & 140 & 130 & 120 & 110 & 100 & 90 & 80 & 70 & 60 & 50 & 40 & 30 & 20 & 10 & 0 & -10\end{array}$

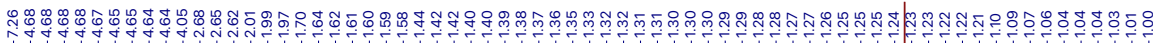

1H NMR $\left(400 \mathrm{MHz}, \mathrm{CDCl}_{3}\right)$<smiles>C=C(C)CCCCC1CCN(C(=O)OC(C)(C)C)CC1</smiles>

$3 q$
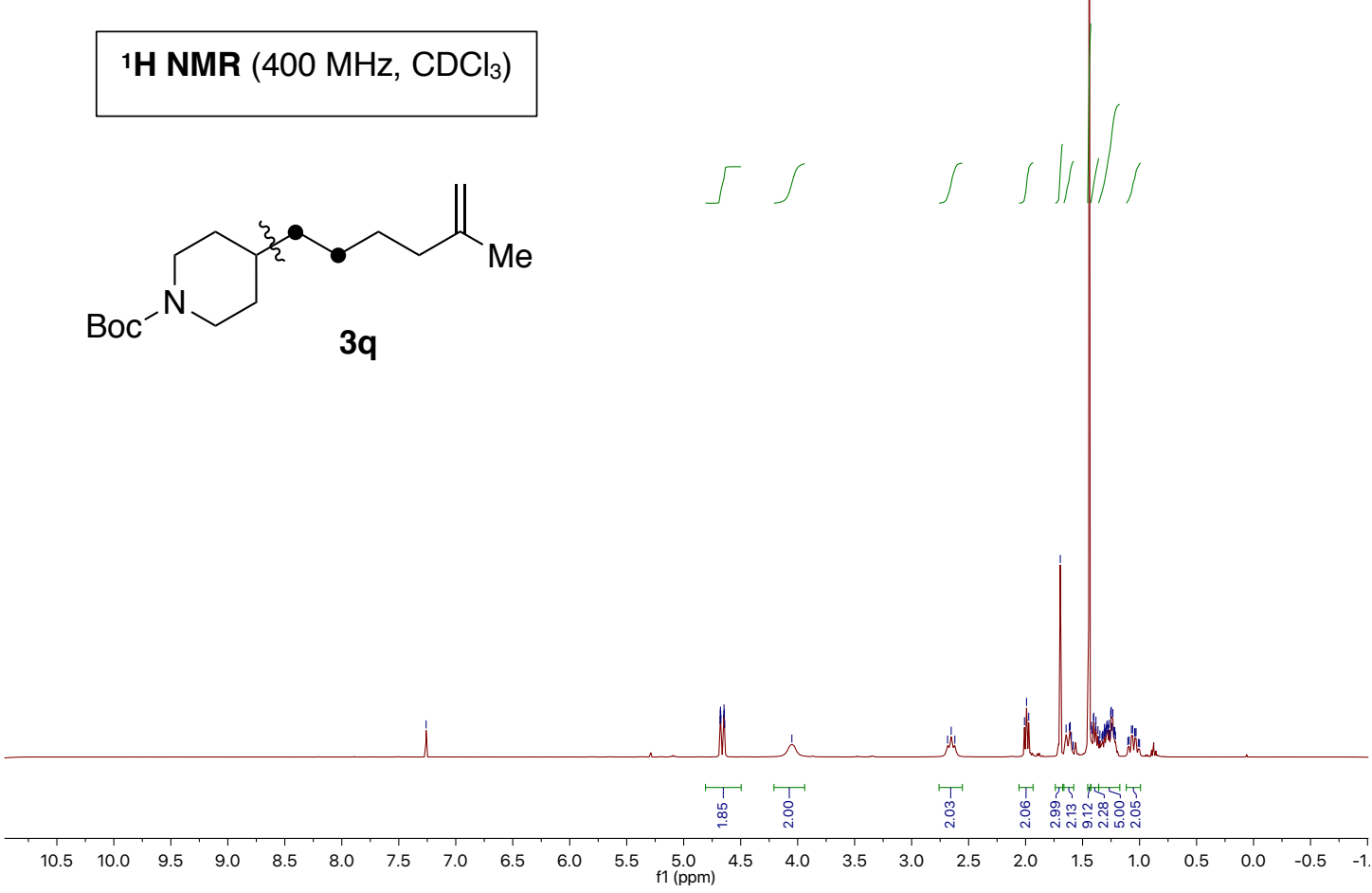

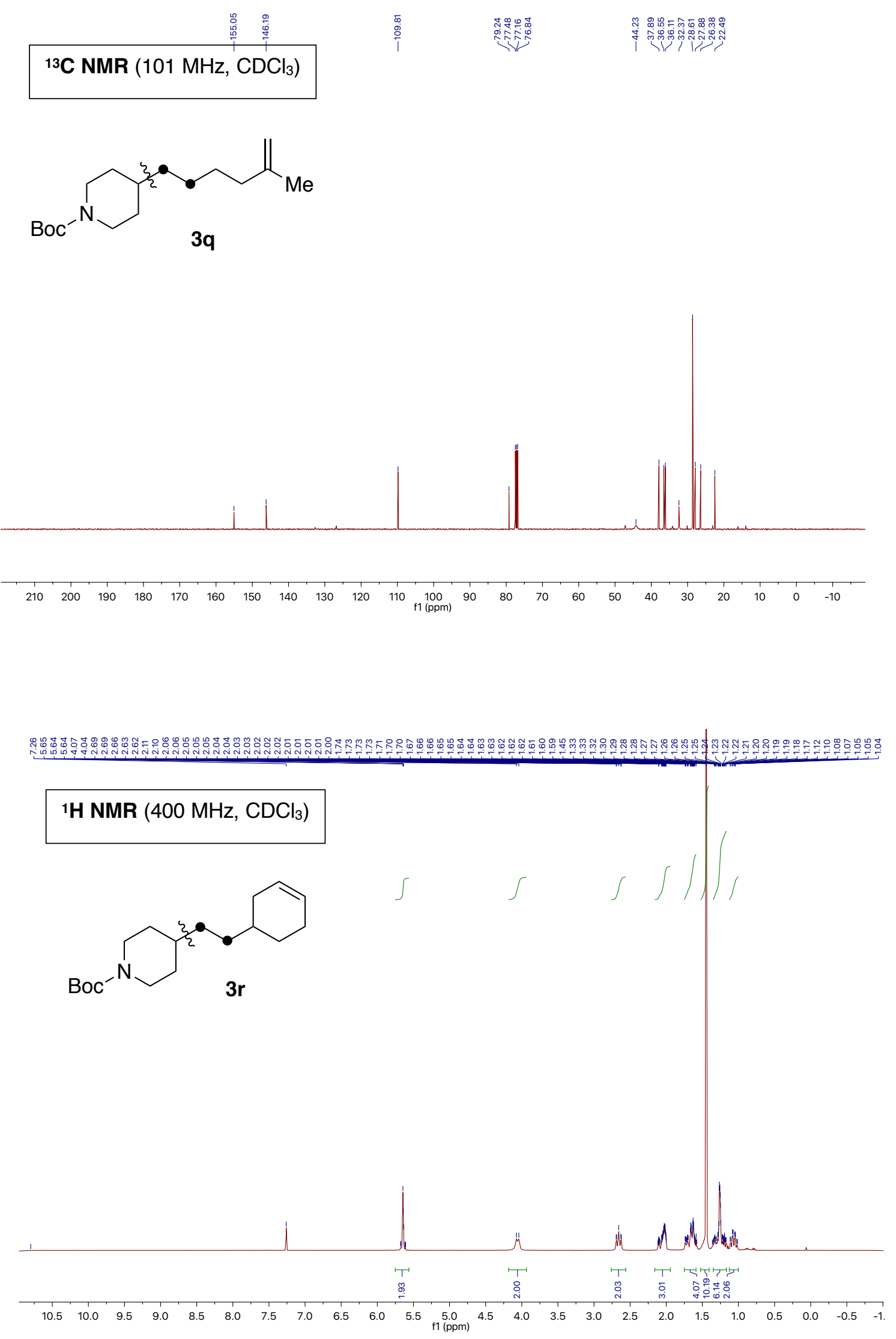


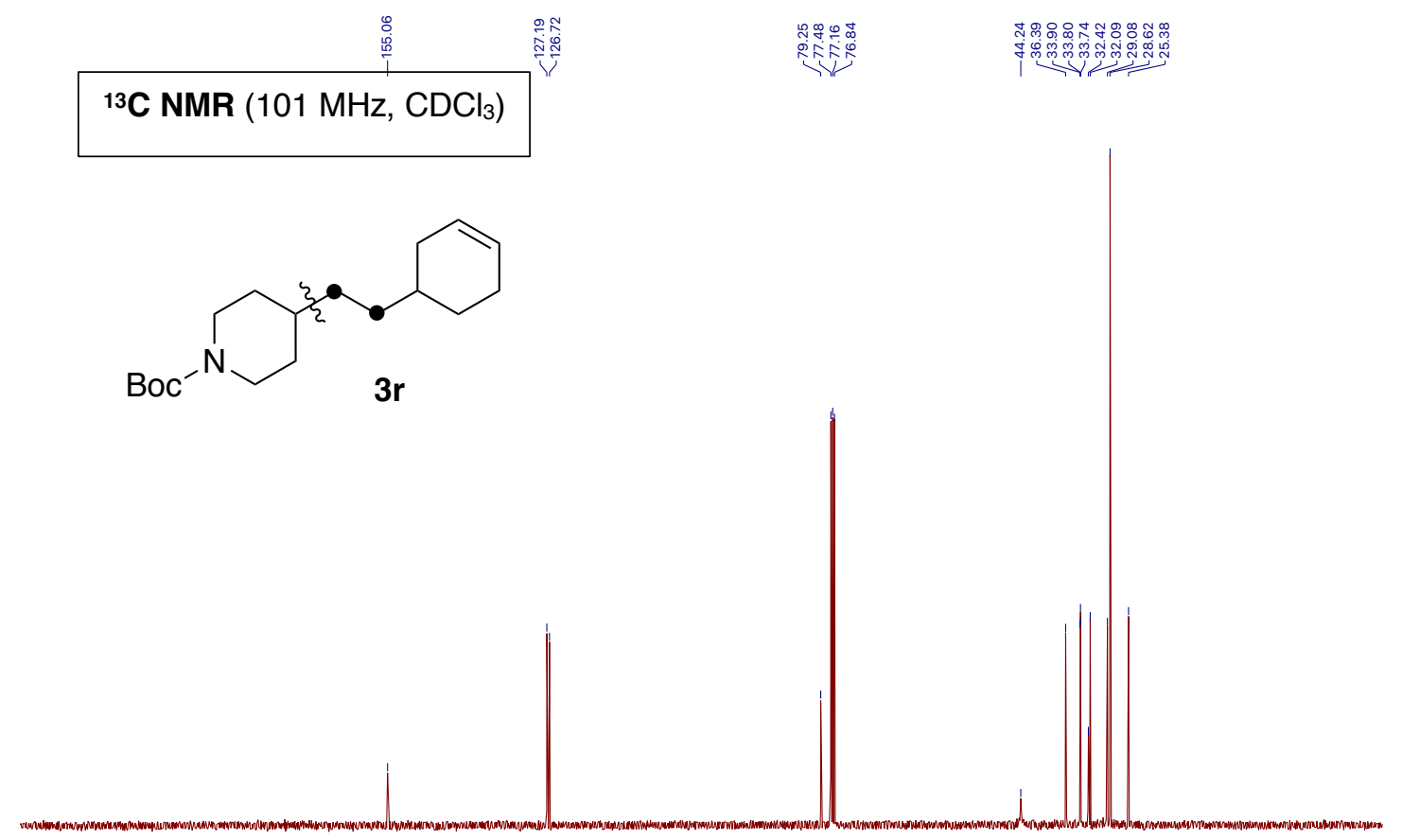

$\begin{array}{lllllllllllllllllllllll}210 & 200 & 190 & 180 & 170 & 160 & 150 & 140 & 130 & 120 & 110 & 100 & 90 & 80 & 70 & 60 & 50 & 40 & 30 & 20 & 10 & 0 & -10\end{array}$

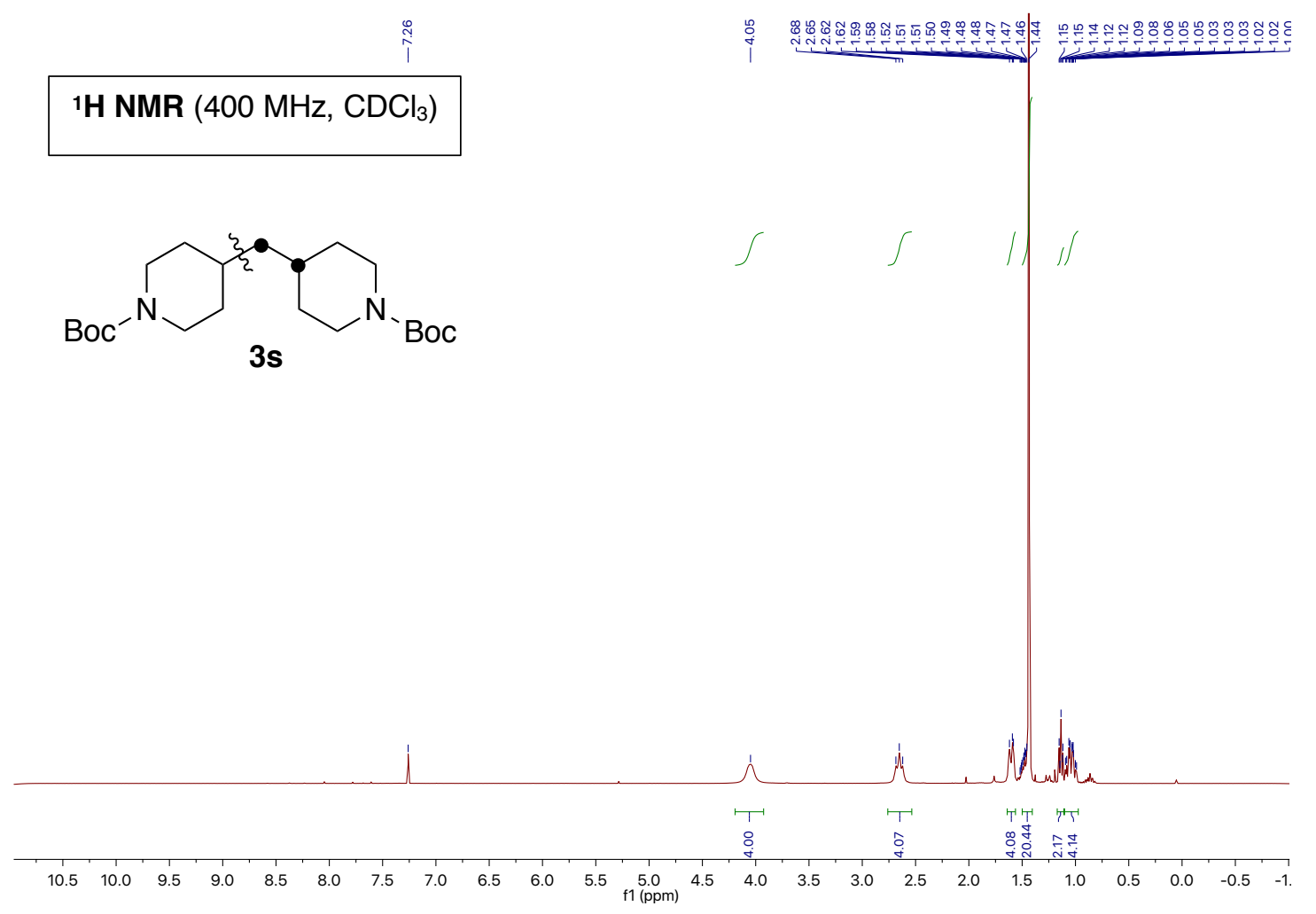




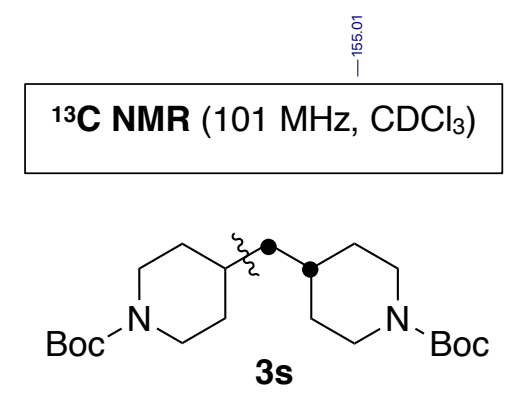

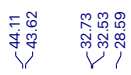

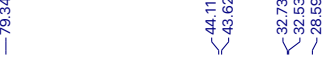
. 


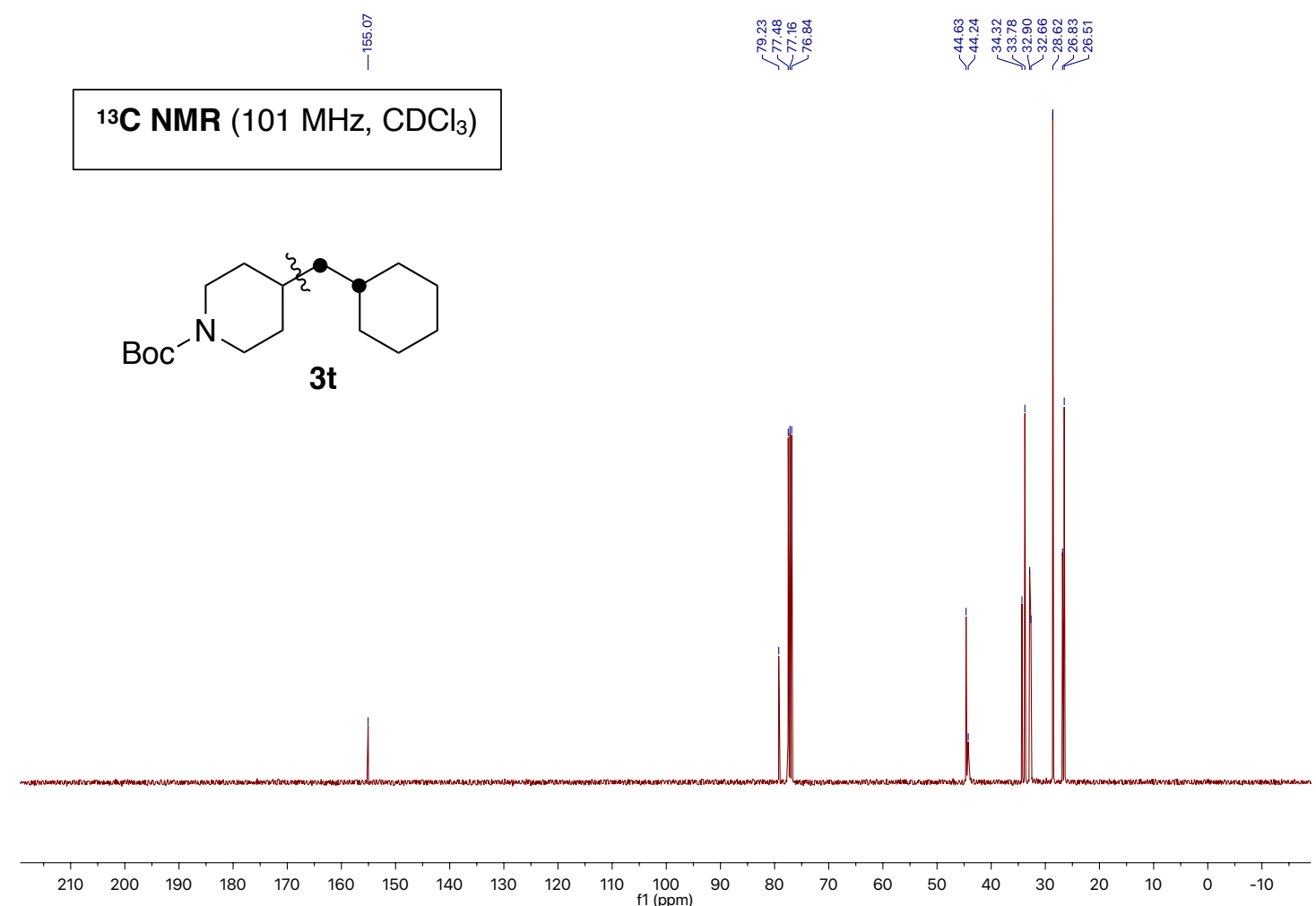

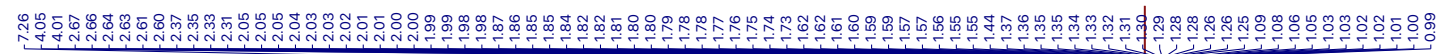

1H NMR (400 MHz, $\mathrm{CDCl}_{3}$ )<smiles>CC(C)(C)OC(=O)N1CCC(CC2CCC2)CC1</smiles>

$3 u$
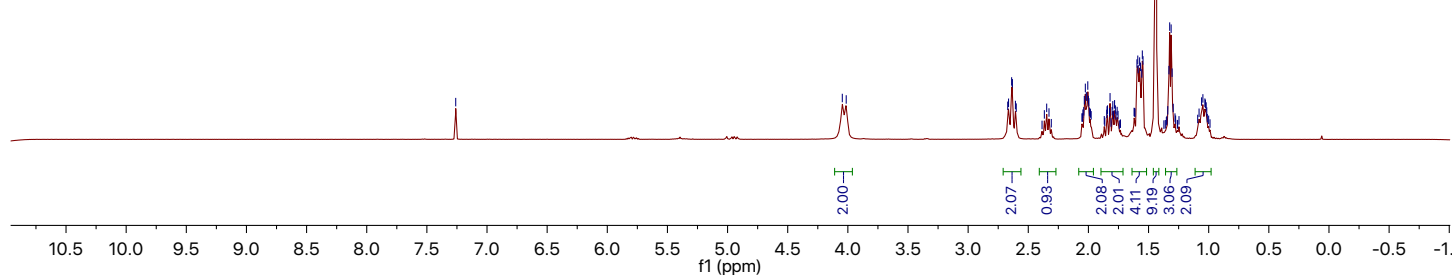


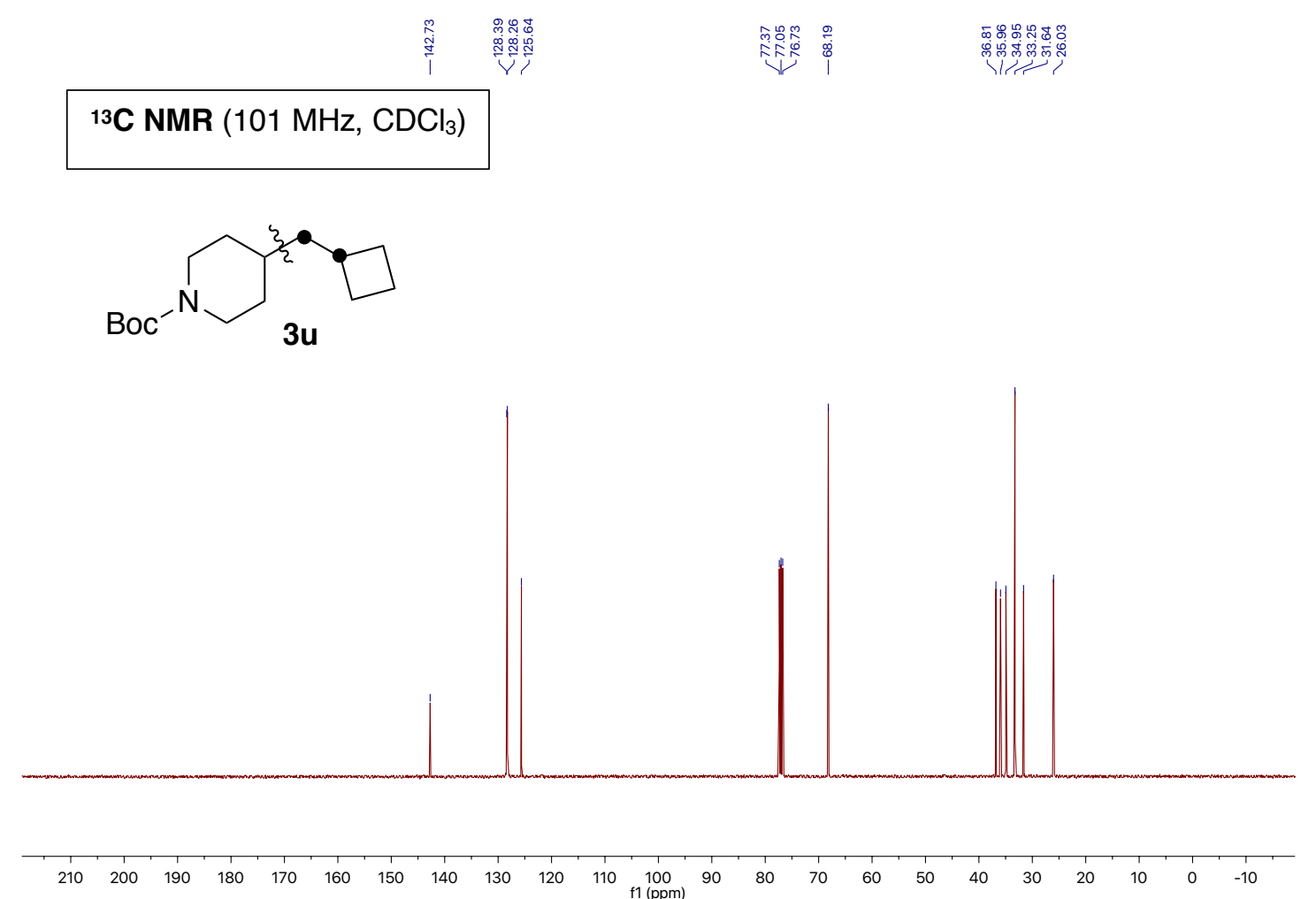

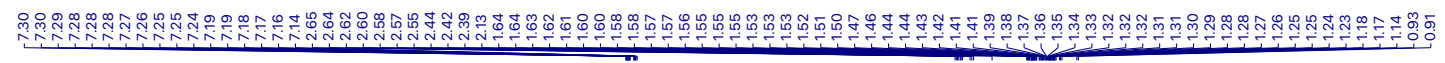

1H NMR (300 MHz, $\left.\mathrm{CDCl}_{3}\right)$<smiles>CC(=O)CCCCCC(C)CCc1ccccc1</smiles>

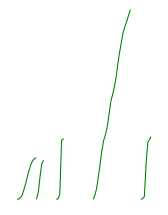




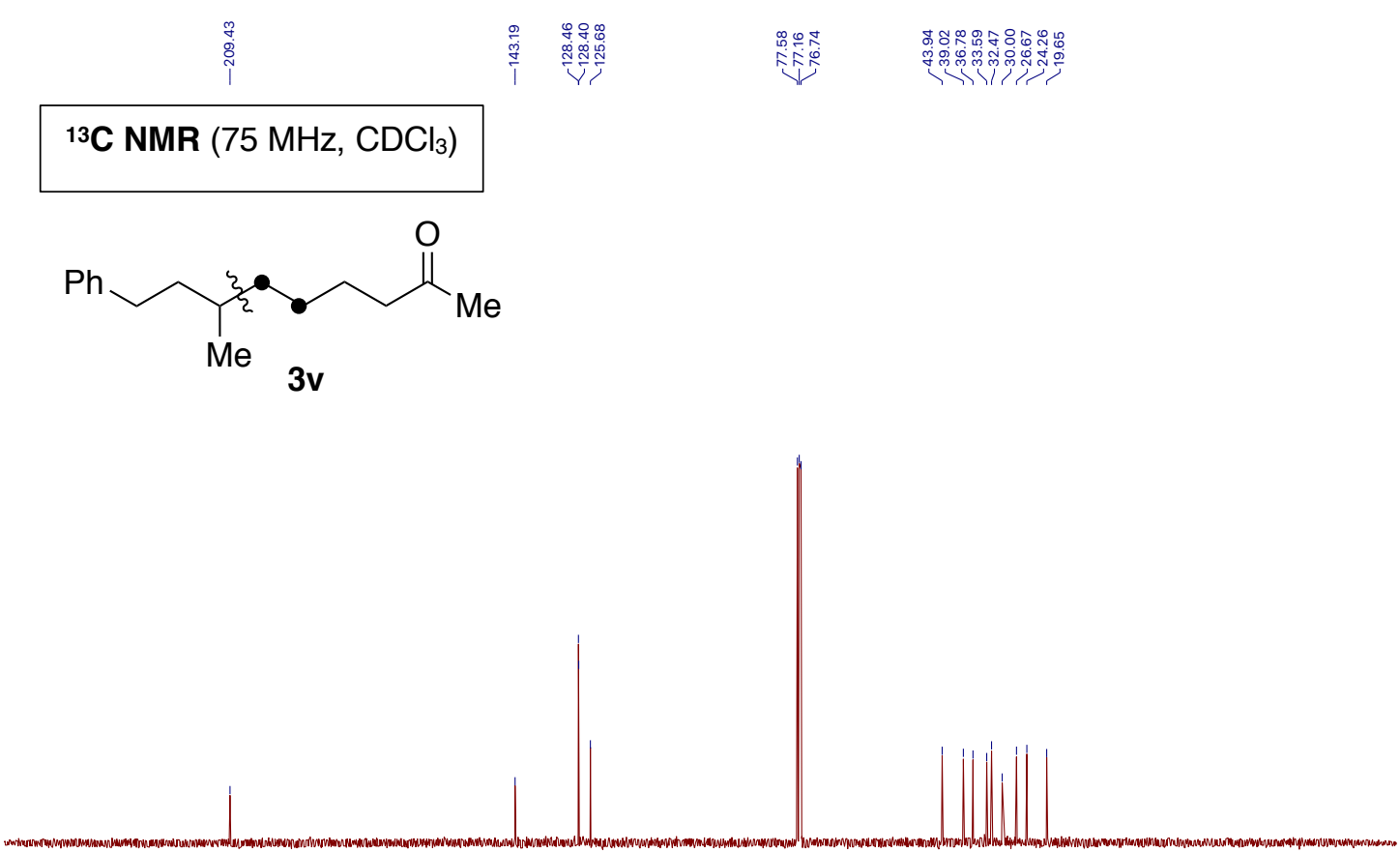

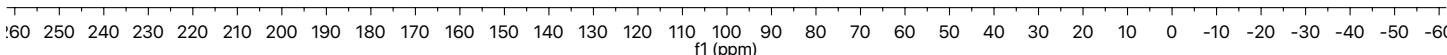

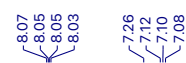

$\underbrace{3}$

1H NMR (400 MHz, $\left.\mathrm{CDCl}_{3}\right)$<smiles>CCC(C)CCCCCOC(=O)c1ccc(F)cc1</smiles>

$3 w$

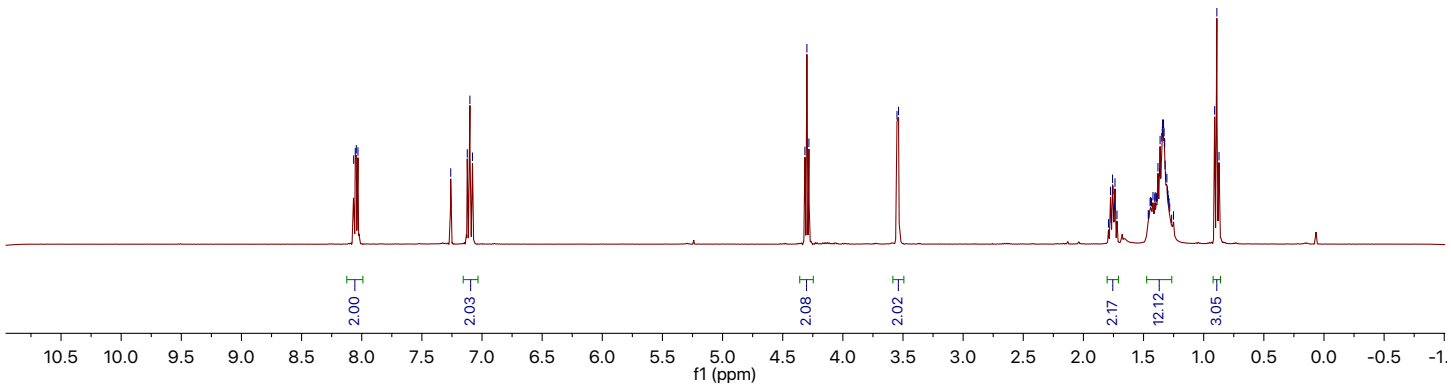




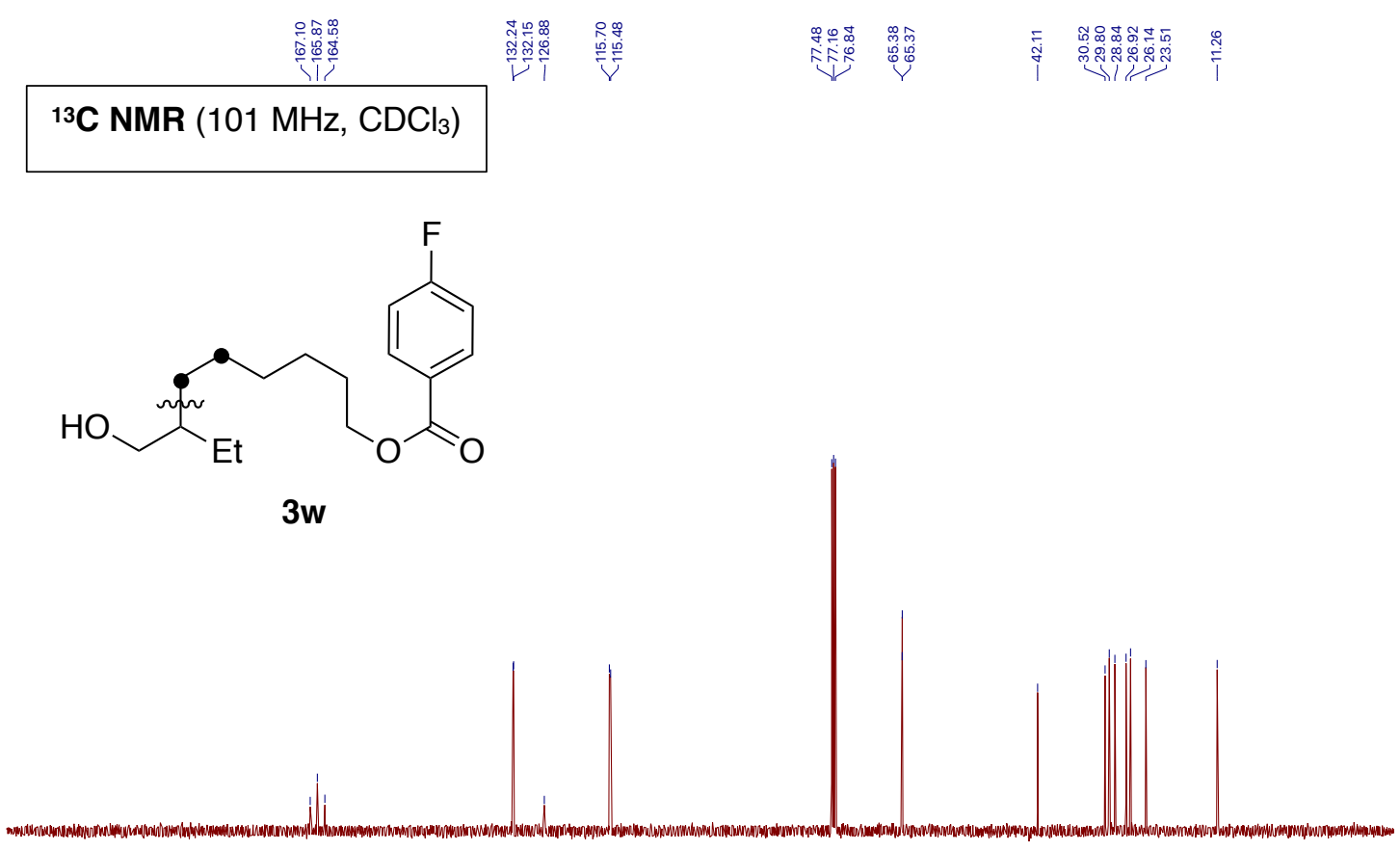

$\begin{array}{lllllllllllllllllllllll}210 & 200 & 190 & 180 & 170 & 160 & 150 & 140 & 130 & 120 & 110 & 100 & 90 & 80 & 70 & 60 & 50 & 40 & 30 & 20 & 10 & 0 & -10\end{array}$

19F NMR (376 MHz, $\left.\mathrm{CDCl}_{3}\right)$

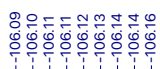<smiles>CC[C@H](C)[C@H](CO)CCCCCOC(=O)c1ccc(F)cc1</smiles>

$3 w$

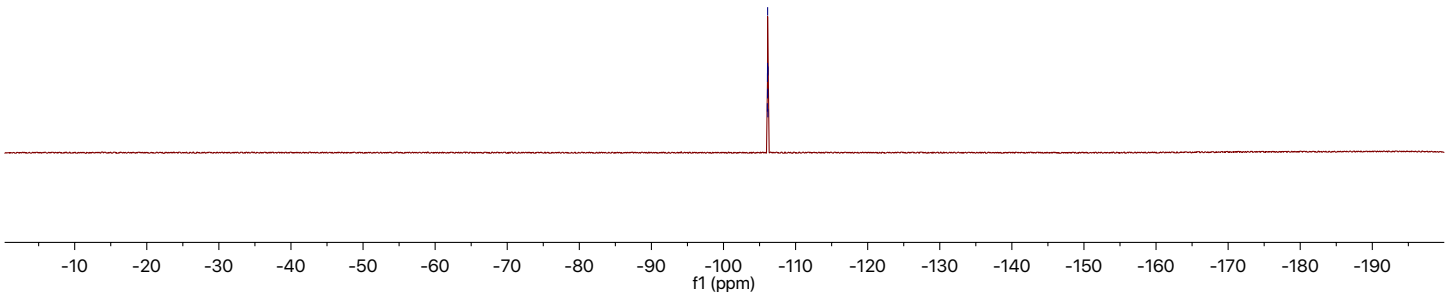



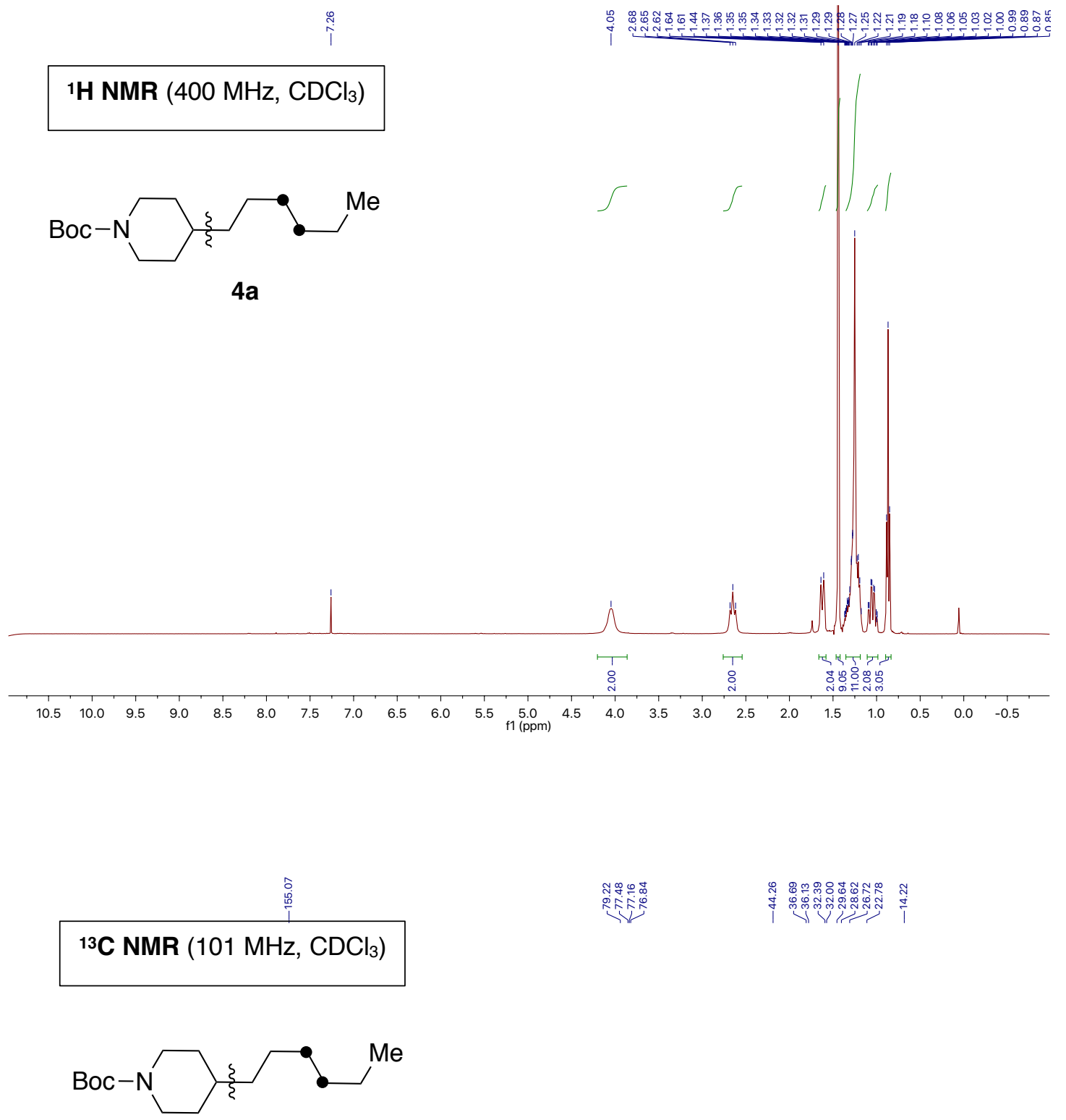

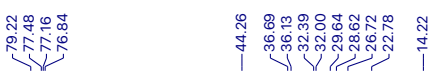

$4 a$

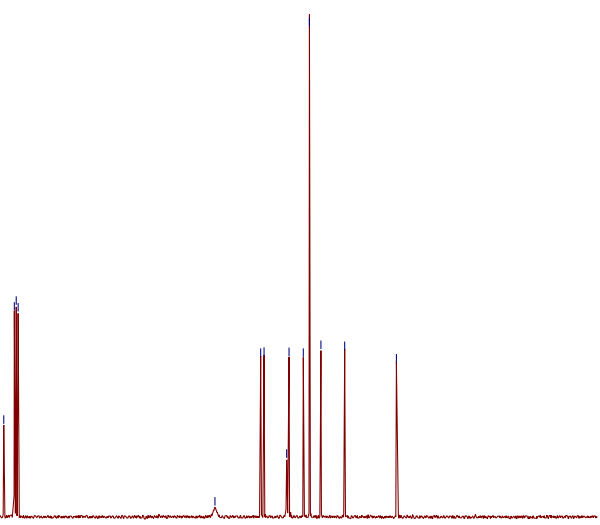

$\begin{array}{lllllllllllllllllllllllllllllllll} & 210 & 200 & 190 & 180 & 170 & 160 & 150 & 140 & 130 & 120 & 110 & 100 & 90 & 80 & 70 & 60 & 50 & 40 & 30 & 20 & 10 & 0 & -10\end{array}$ 

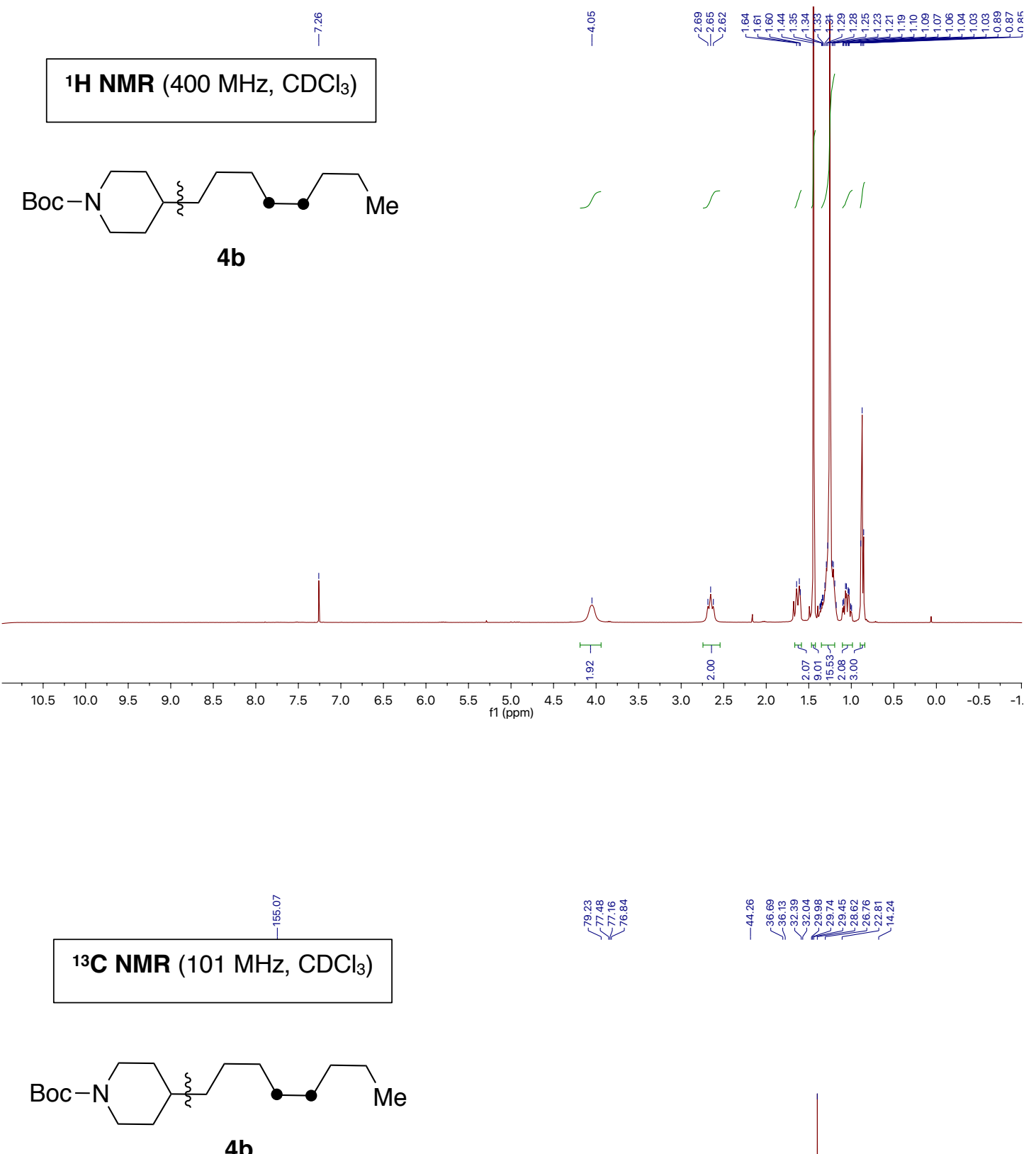

4b

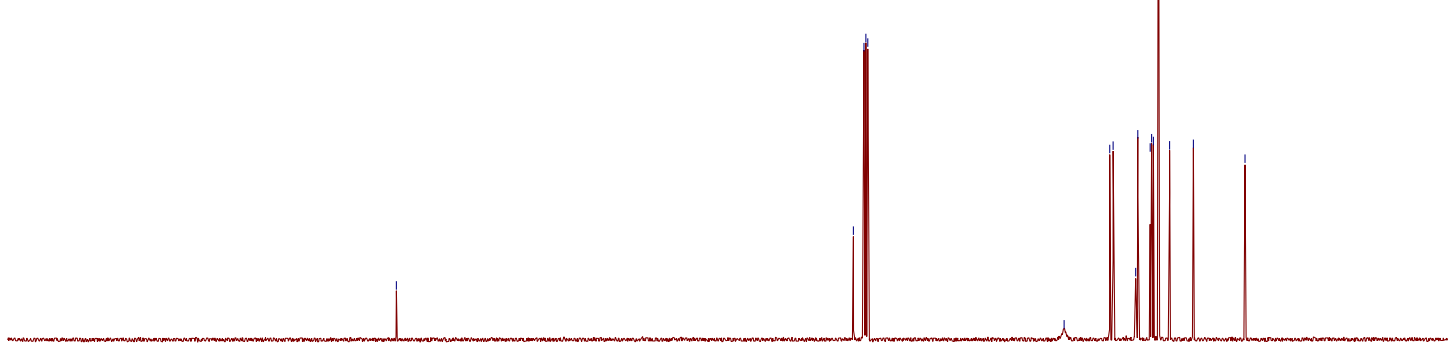

$\begin{array}{lllllllllllllllllllllllllll} & 210 & 200 & 190 & 180 & 170 & 160 & 150 & 140 & 130 & 120 & 110 & 100 & 90 & 80 & 70 & 60 & 50 & 40 & 30 & 20 & 10 & 0 & -10\end{array}$ 

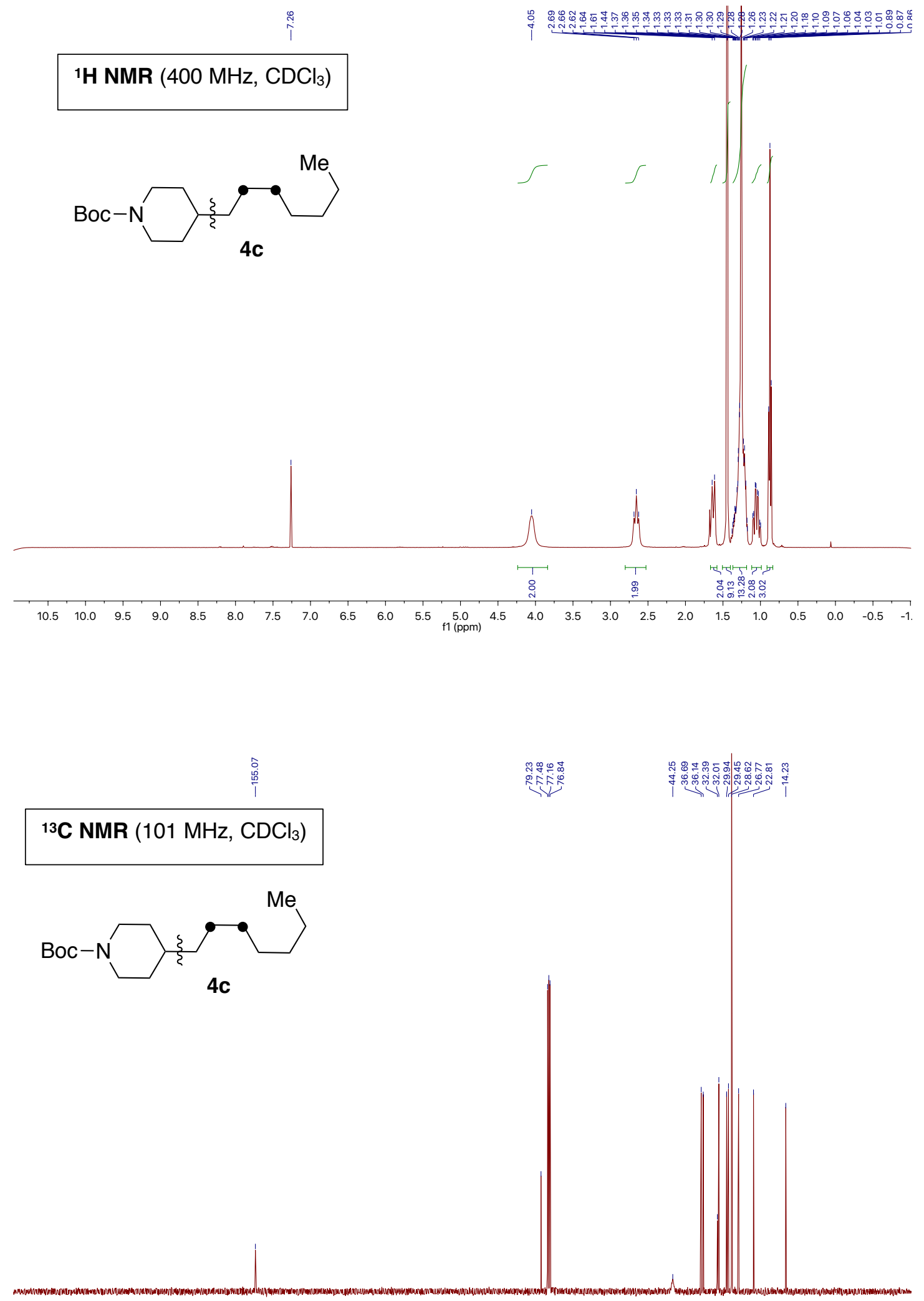

$\begin{array}{lllllllllllllllllllllllllllll}210 & 200 & 190 & 180 & 170 & 160 & 150 & 140 & 130 & 120 & 110 & \begin{array}{c}100 \\ \mathrm{f} 1(\mathrm{ppm})\end{array} & 90 & 80 & 70 & 60 & 50 & 40 & 30 & 20 & 10 & 0 & -10\end{array}$ 


\section{H NMR $\left(400 \mathrm{MHz}, \mathrm{CDCl}_{3}\right)$}
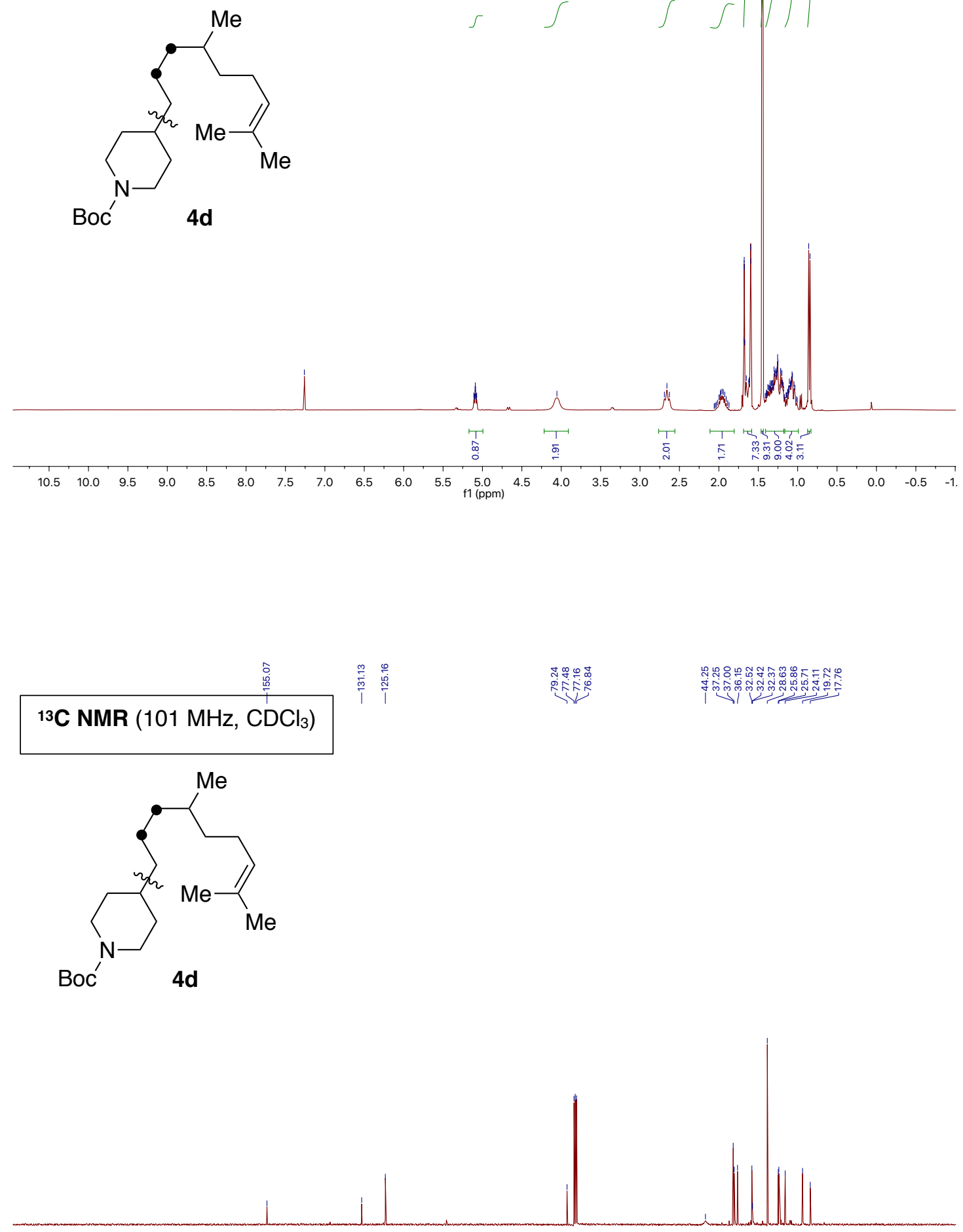

$\begin{array}{lllllllllllllllllllllllllllll}1 & 210 & 200 & 190 & 180 & 170 & 160 & 150 & 140 & 130 & 120 & 110 & \begin{array}{c}100 \\ \mathrm{f}(\mathrm{ppm})\end{array} & 90 & 80 & 70 & 60 & 50 & 40 & 30 & 20 & 10 & 0 & -10\end{array}$ 

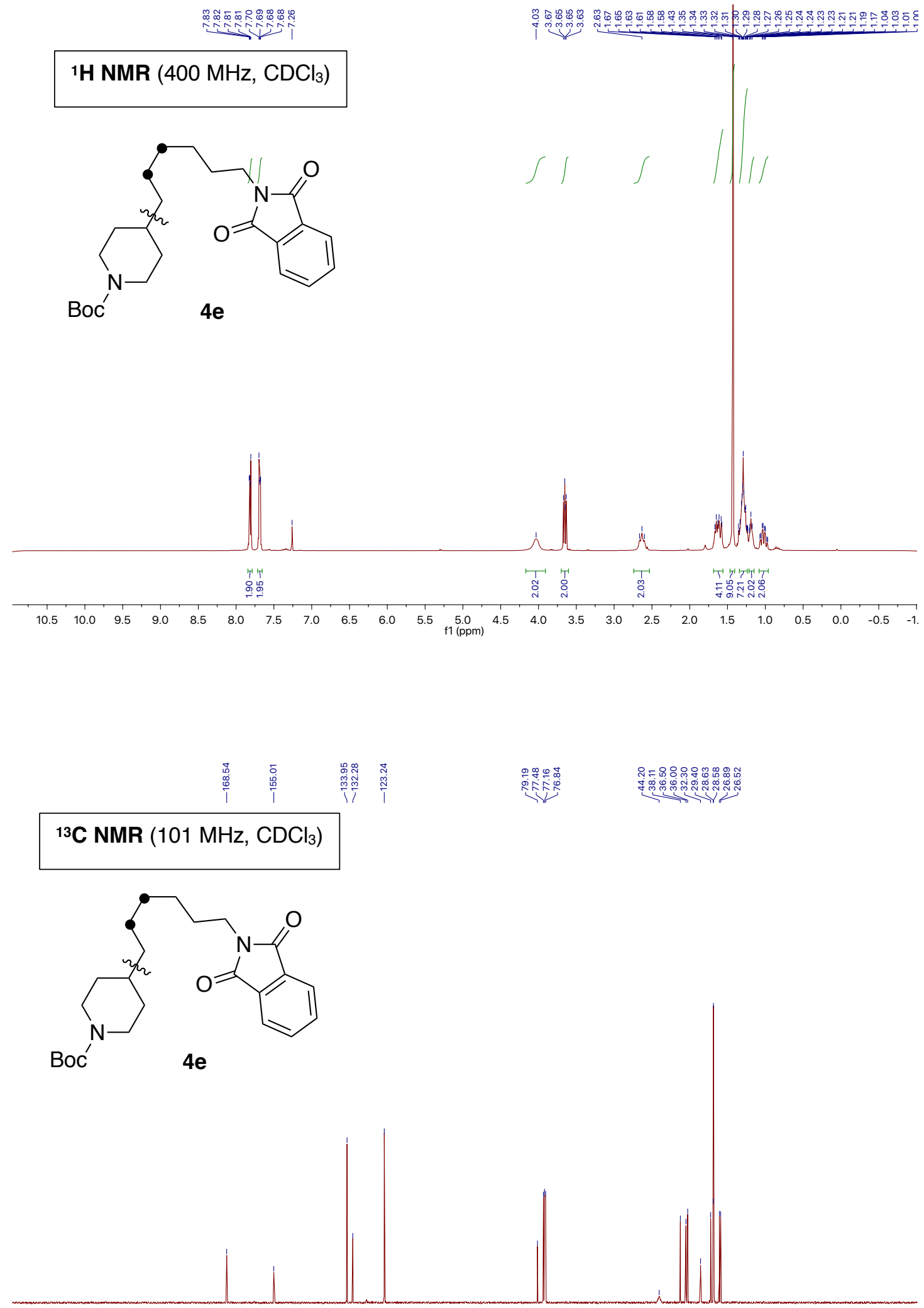

\begin{tabular}{lllllllllllllllllllllllllllllllllll}
\hline 30 & 220 & 210 & 200 & 190 & 180 & 170 & 160 & 150 & 140 & 130 & 120 & 110 & 100 & 90 & 10 & 70 & 60 & 50 & 40 & 30 & 20 & 10 & 0 & -10 & -20
\end{tabular} 

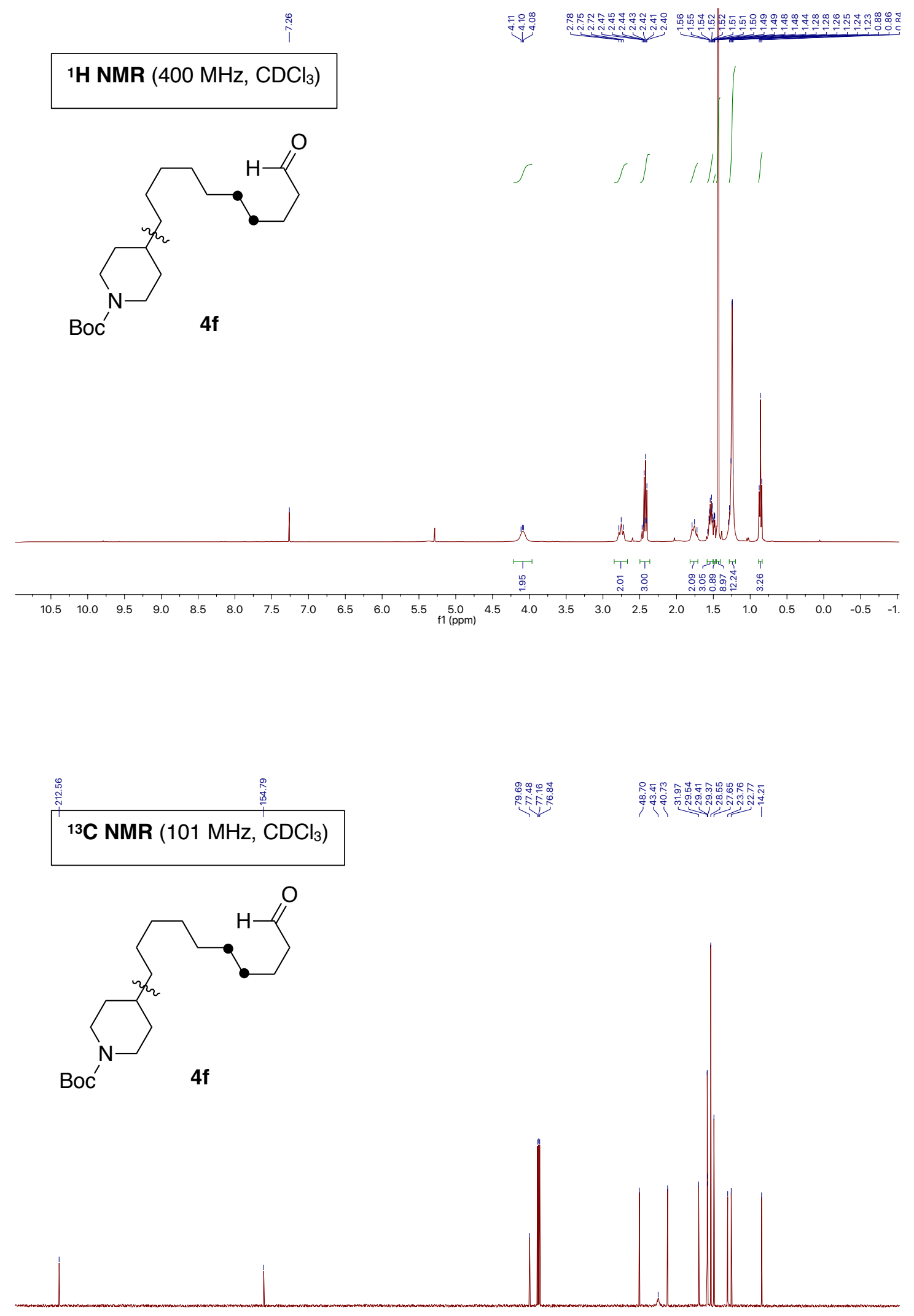

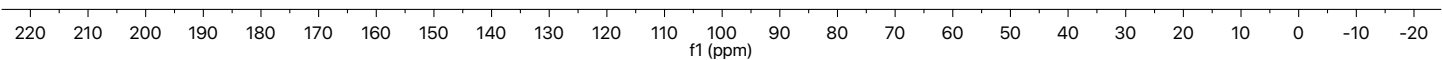


1H NMR (400 MHz, $\left.\mathrm{CDCl}_{3}\right)$
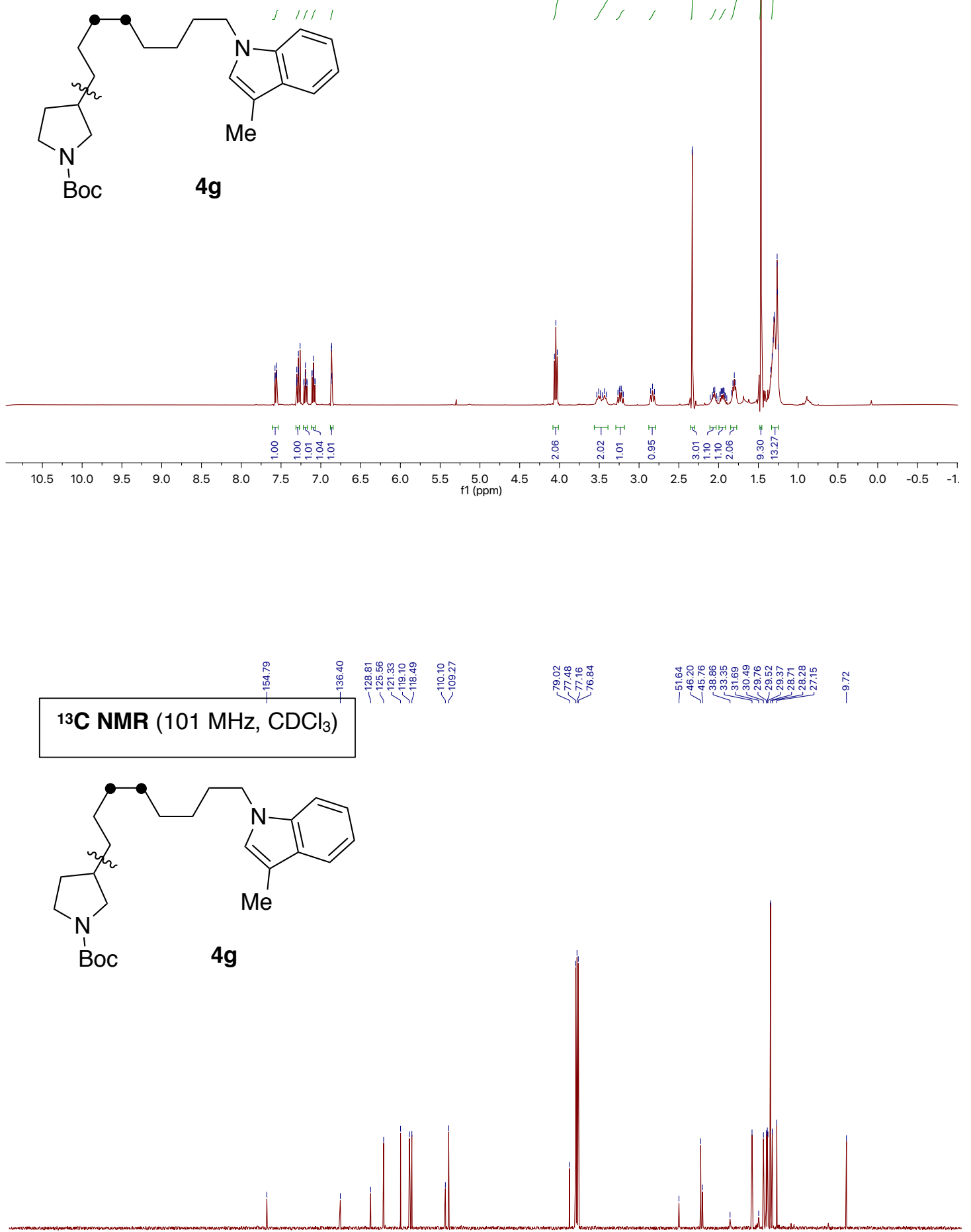

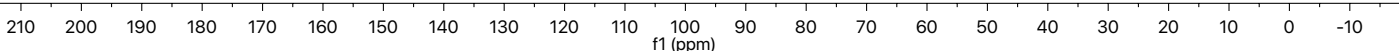




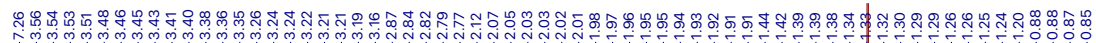

1H NMR (400 MHz, $\left.\mathrm{CDCl}_{3}\right)$

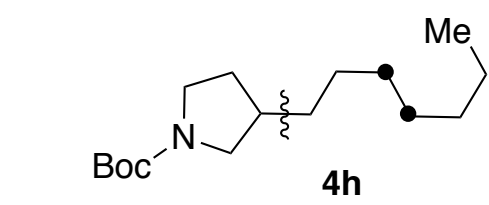

Whe wh
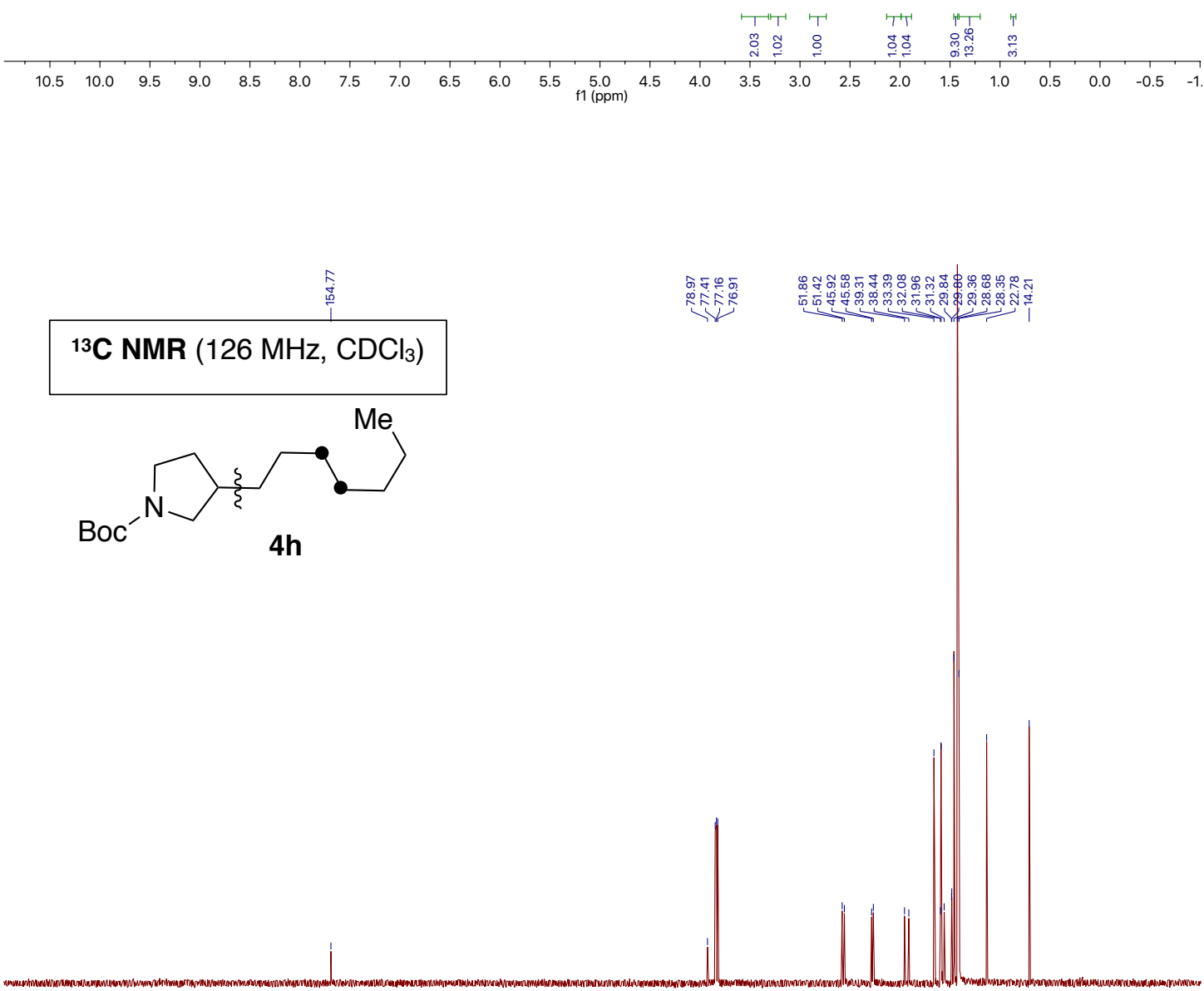

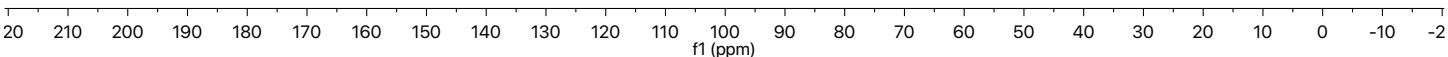




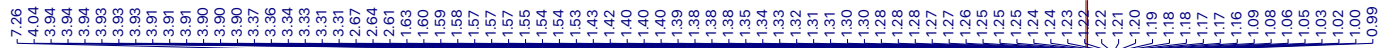

1H NMR $\left(400 \mathrm{MHz}, \mathrm{CDCl}_{3}\right)$
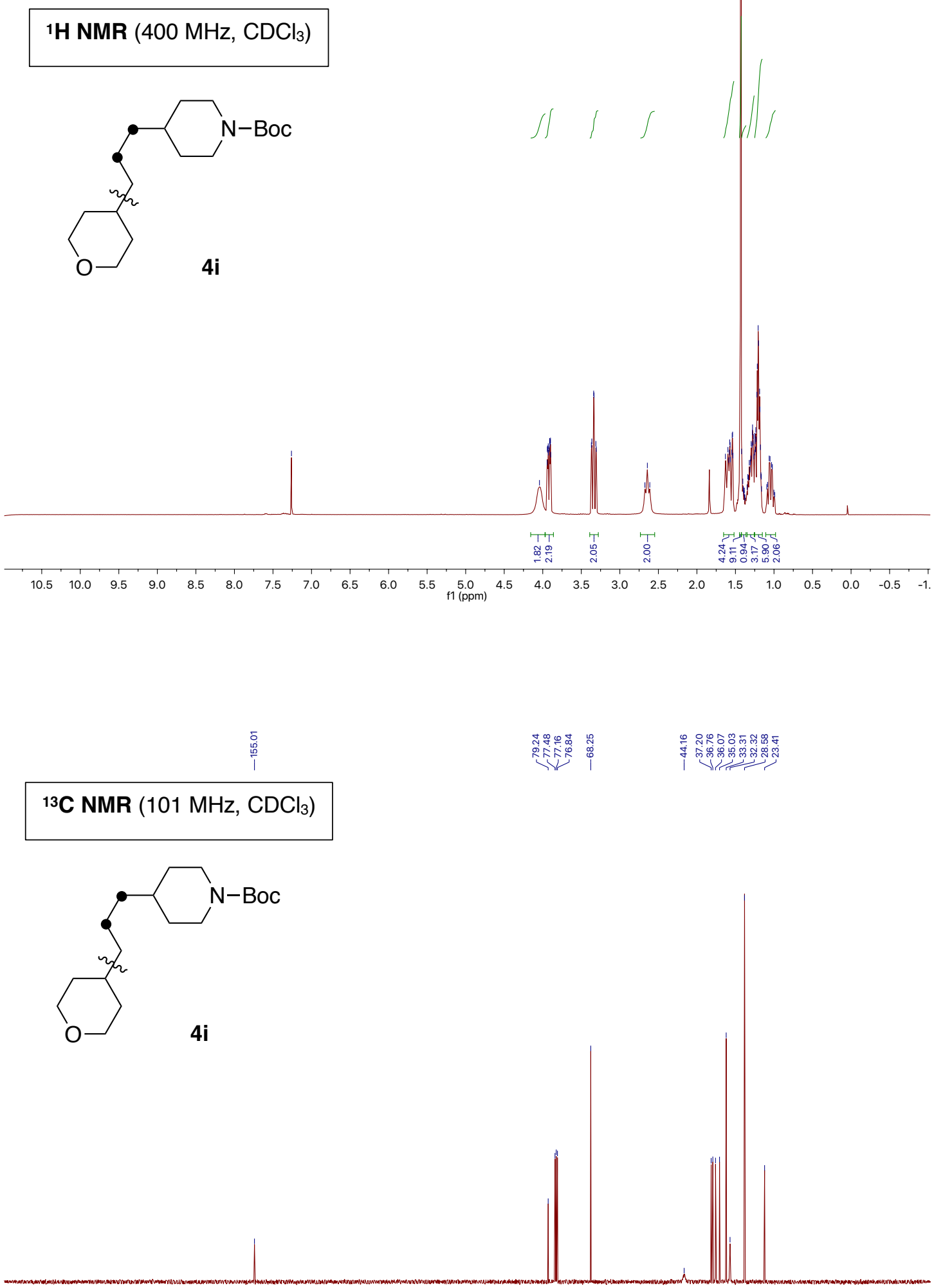

$\begin{array}{lllllllllllllllllllllllllll}210 & 200 & 190 & 180 & 170 & 160 & 150 & 140 & 130 & 120 & 110 & \begin{array}{c}100 \\ \mathrm{f} 1(\mathrm{pm})\end{array} & 90 & 80 & 70 & 60 & 50 & 40 & 30 & 20 & 10 & 0 & -10\end{array}$ 


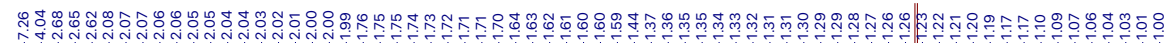
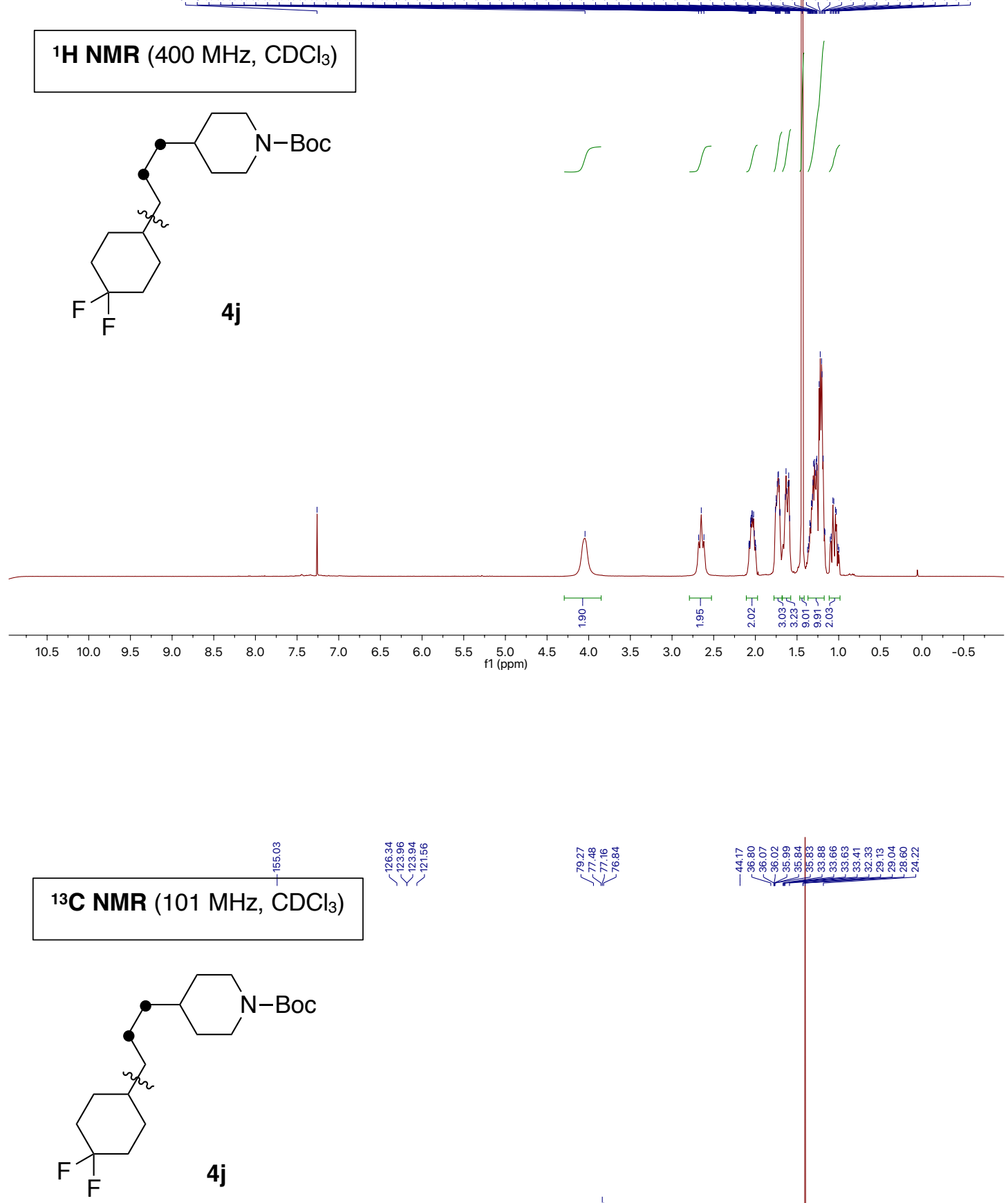

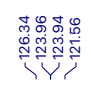

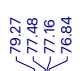

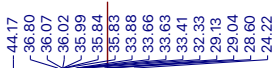

Hi

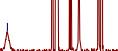

$\begin{array}{llllllllllll}210 & 200 & 190 & 180 & 170 & 160 & 150 & 140 & 130 & 120 & 110 & \begin{array}{l}100 \\ \mathrm{f} 1(\mathrm{ppm})\end{array}\end{array}$ 
19F NMR (376 MHz, $\left.\mathrm{CDCl}_{3}\right)$

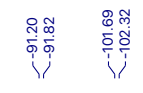<smiles>CC(=O)N1CCC(CCCC2CCC(F)(F)CC2)CC1</smiles>

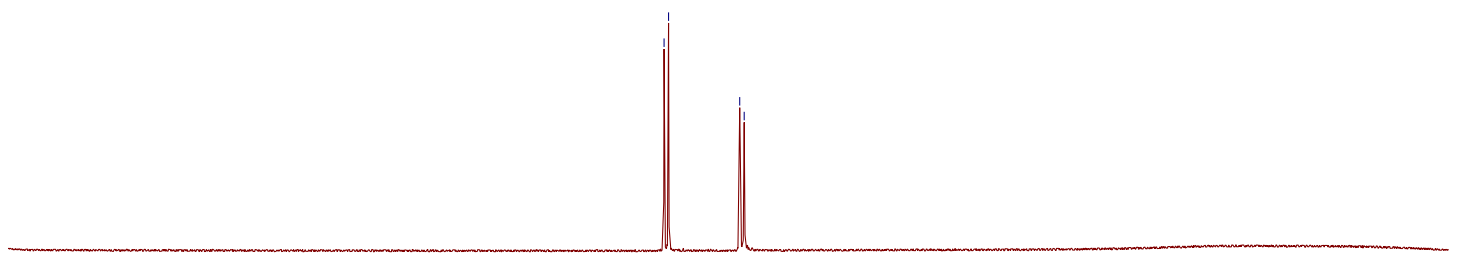

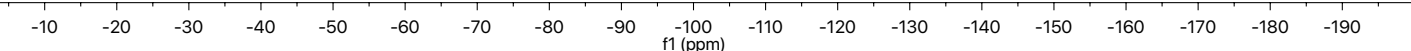

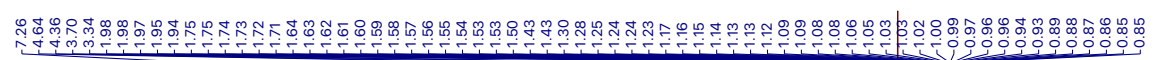

1H NMR (400 MHz, $\left.\mathrm{CDCl}_{3}\right)$
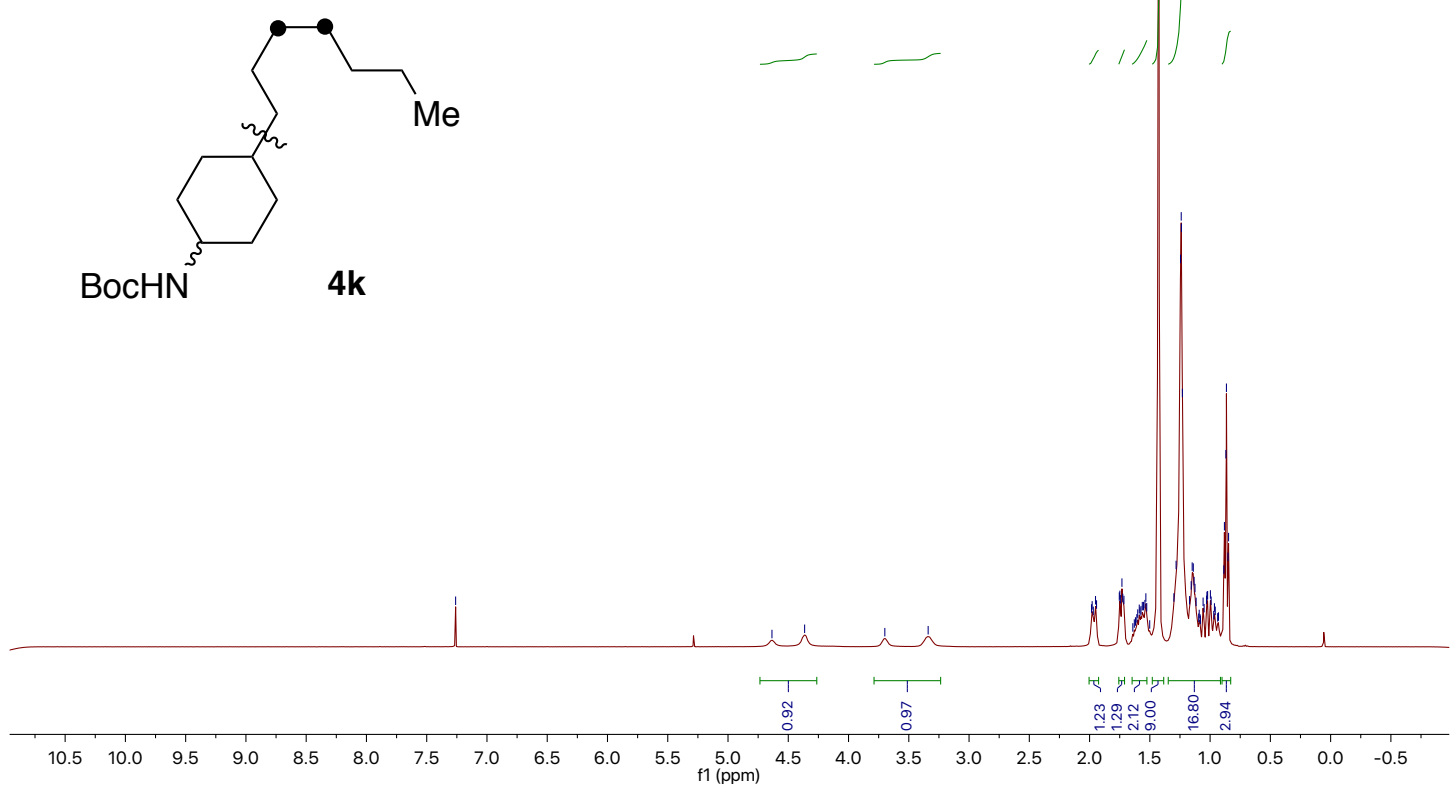


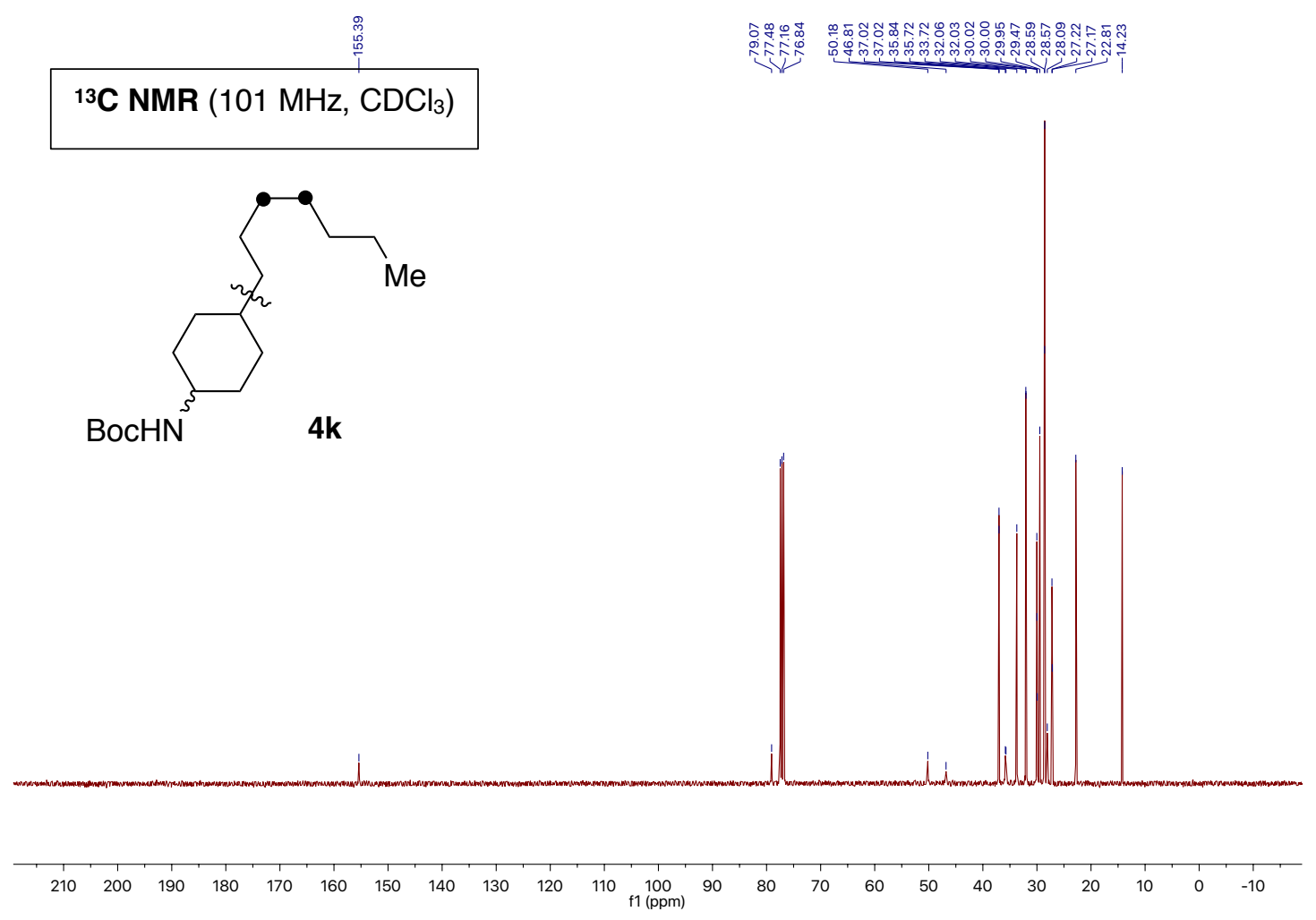

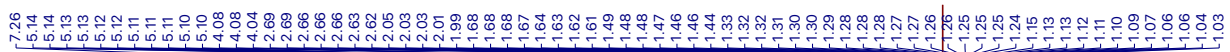

1H NMR (400 MHz, $\mathrm{CDCl}_{3}$ )

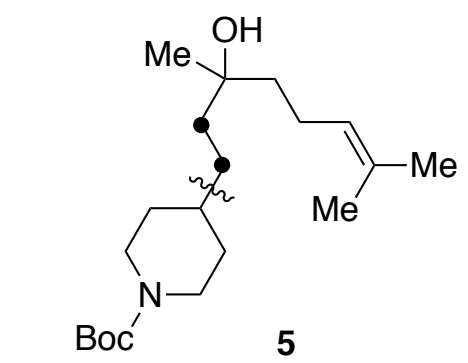




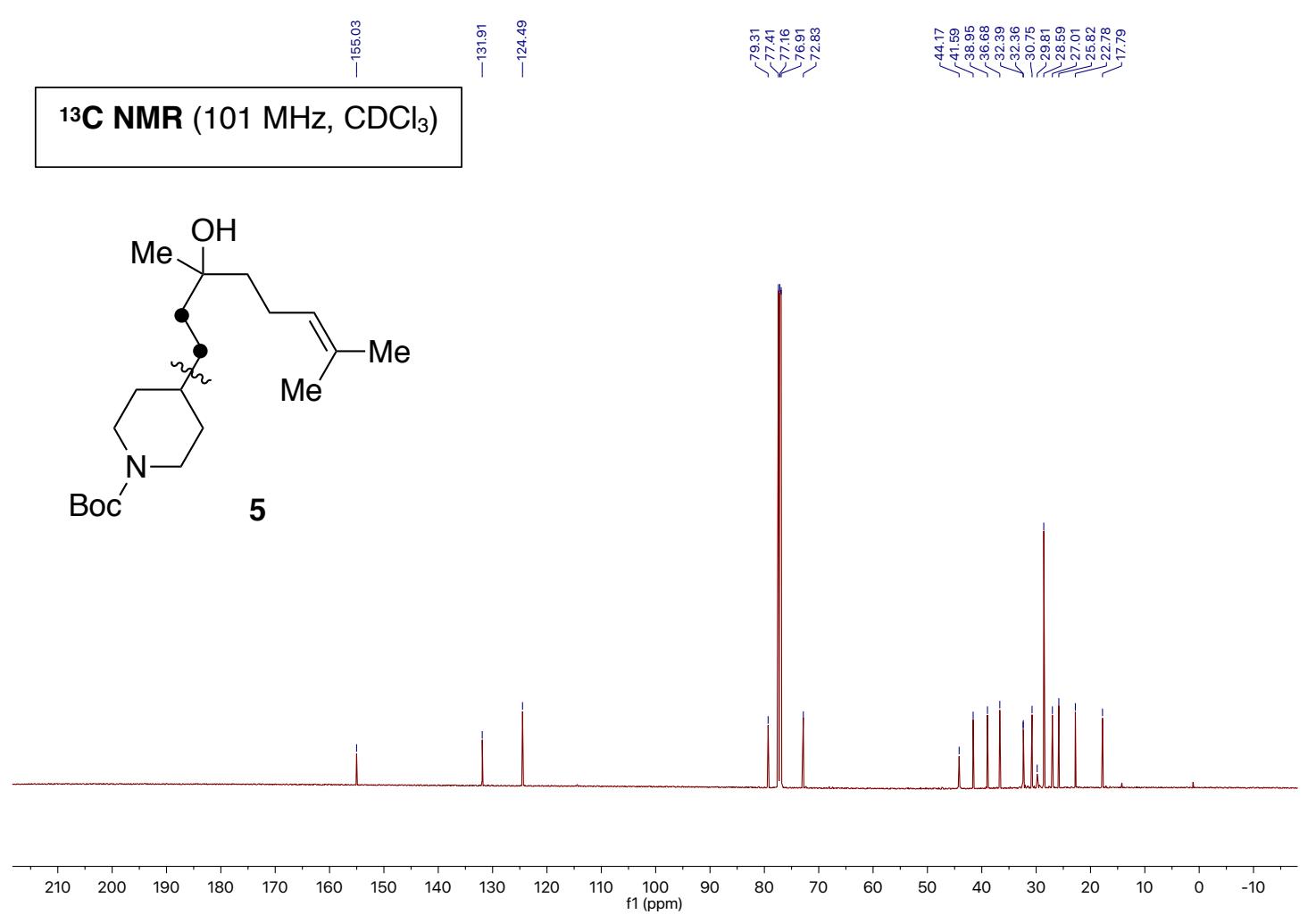

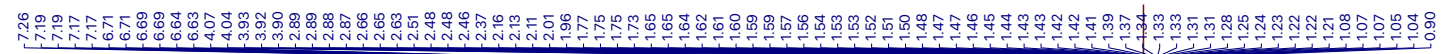

1H NMR $\left(400 \mathrm{MHz}, \mathrm{CDCl}_{3}\right)$

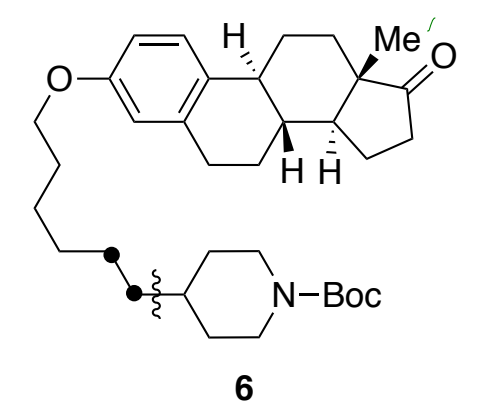




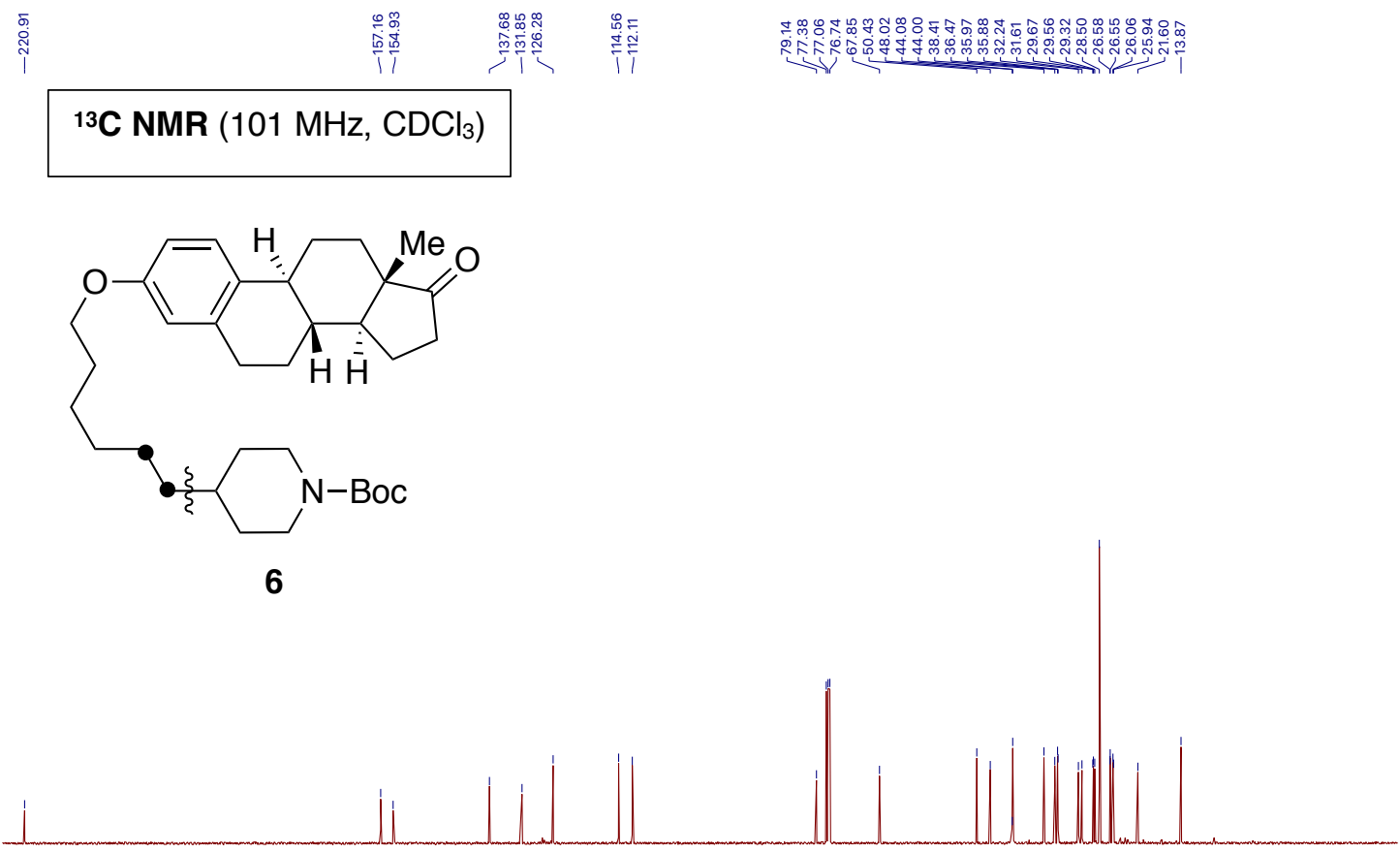

$\begin{array}{lllllllllllllllllllllllllllllllllll}220 & 210 & 200 & 190 & 180 & 170 & 160 & 150 & 140 & 130 & 120 & 110 & 100 & 90 & 80 & 70 & 60 & 50 & 40 & 30 & 20 & 10 & 0 & -10 & -20\end{array}$

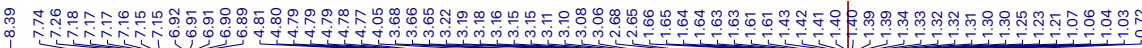

1H NMR (400 MHz, $\left.\mathrm{CDCl}_{3}\right)$<smiles>CC(C)(C)CC(Cc1ccc(Cl)cc1)n1cncn1</smiles>

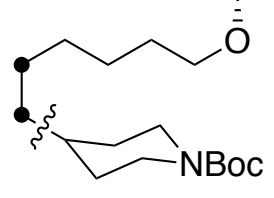

7

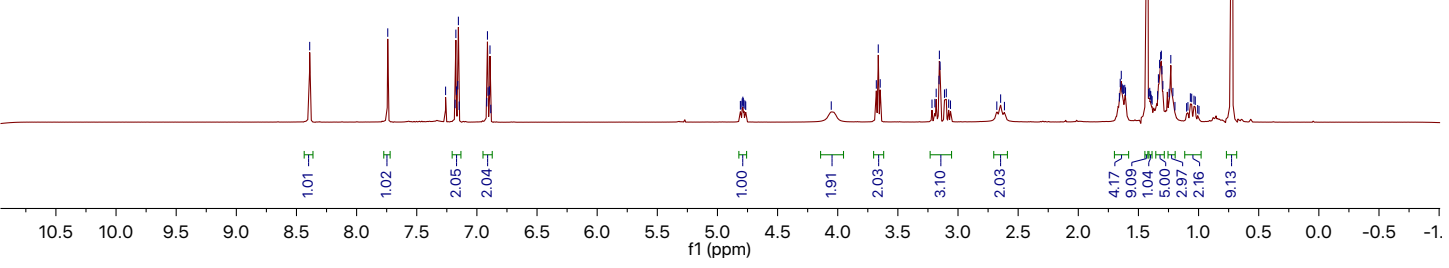




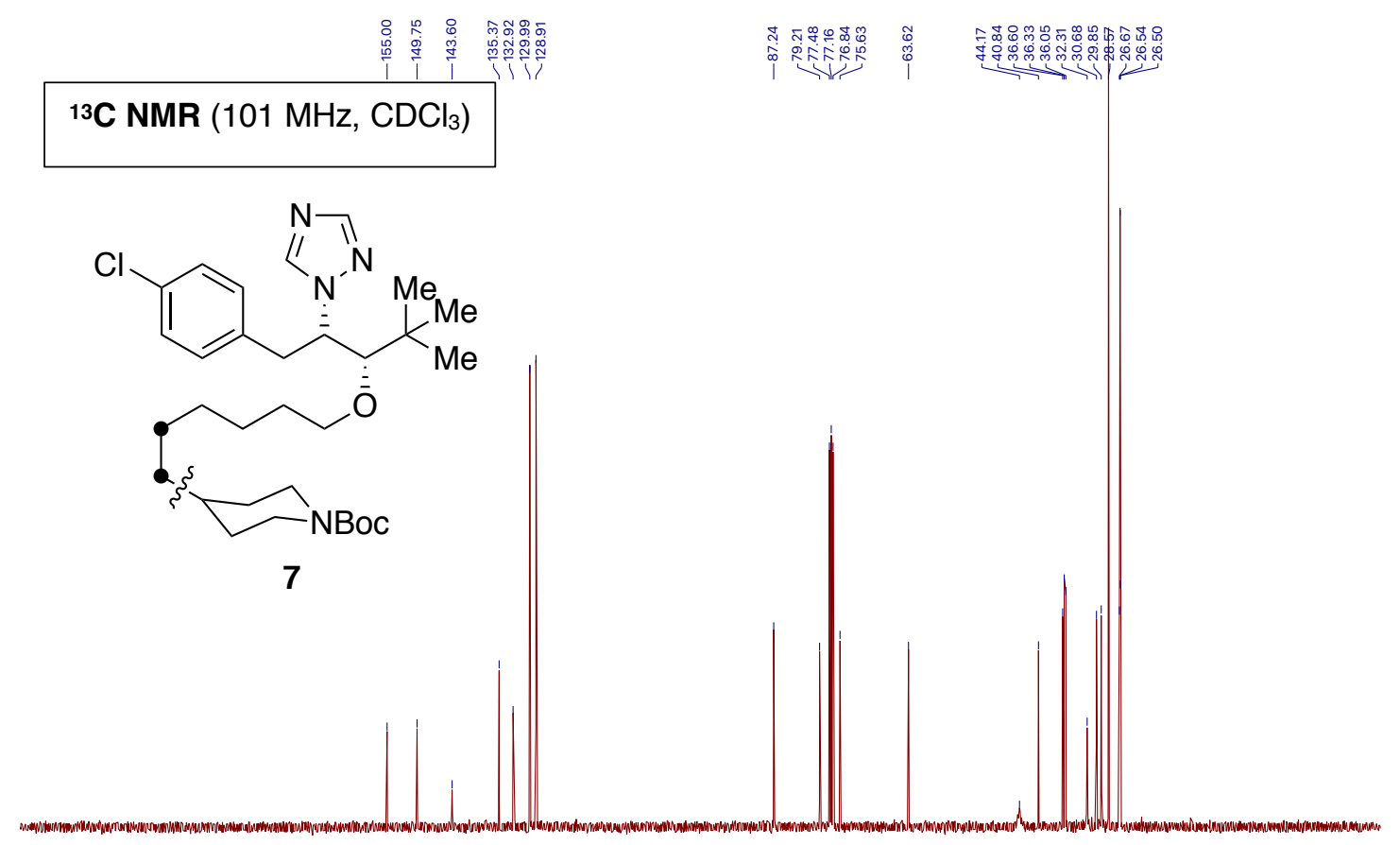

$\begin{array}{lllllllllllllllllllllllllllllllll}210 & 200 & 190 & 180 & 170 & 160 & 150 & 140 & 130 & 120 & 110 & 100 & 90 & 80 & 70 & 60 & 50 & 40 & 30 & 20 & 10 & 0 & -10\end{array}$

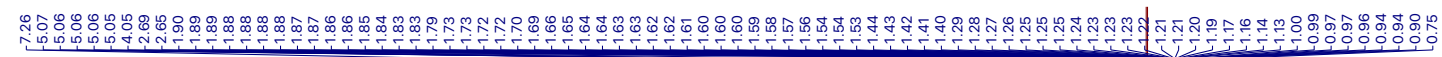

1H NMR (400 MHz, $\mathrm{CDCl}_{3}$ )
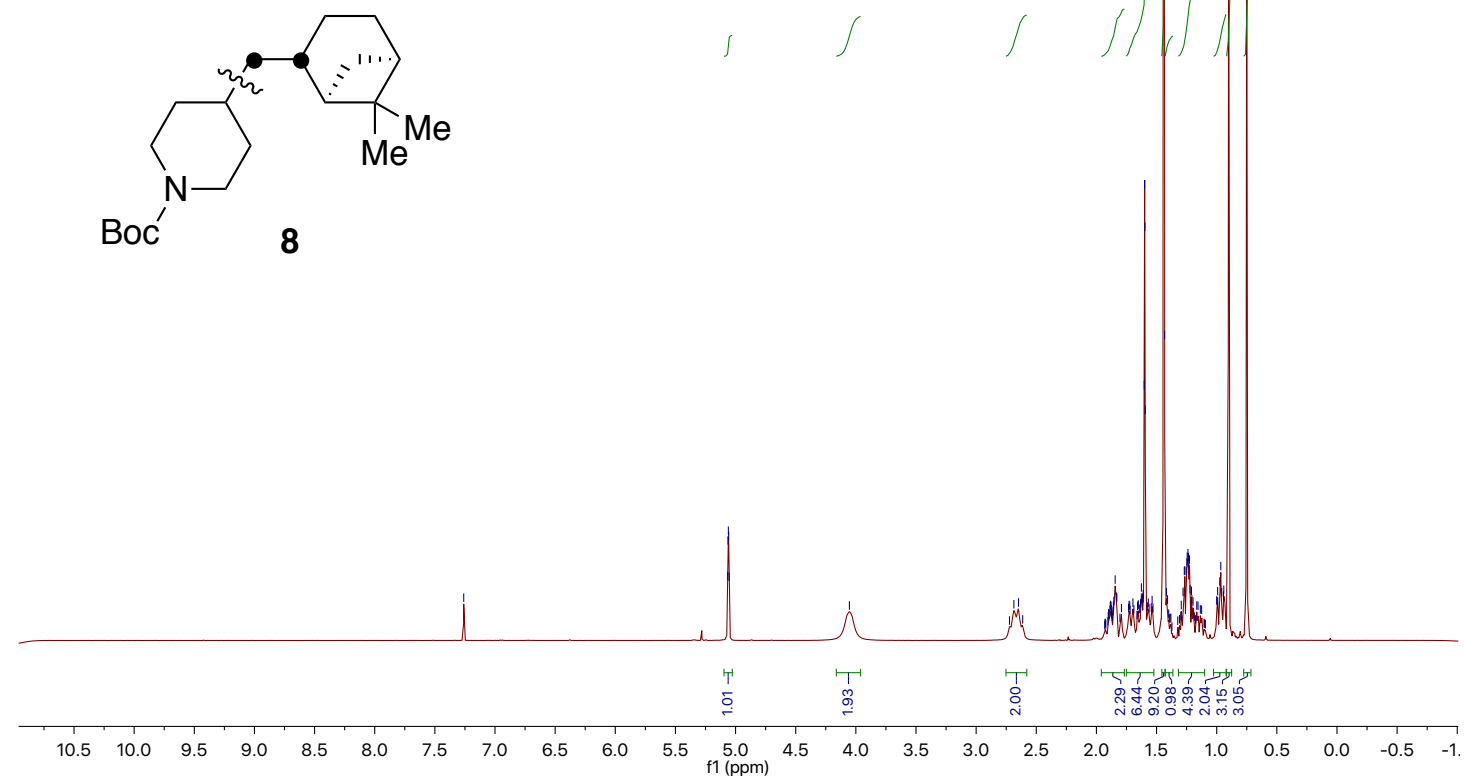


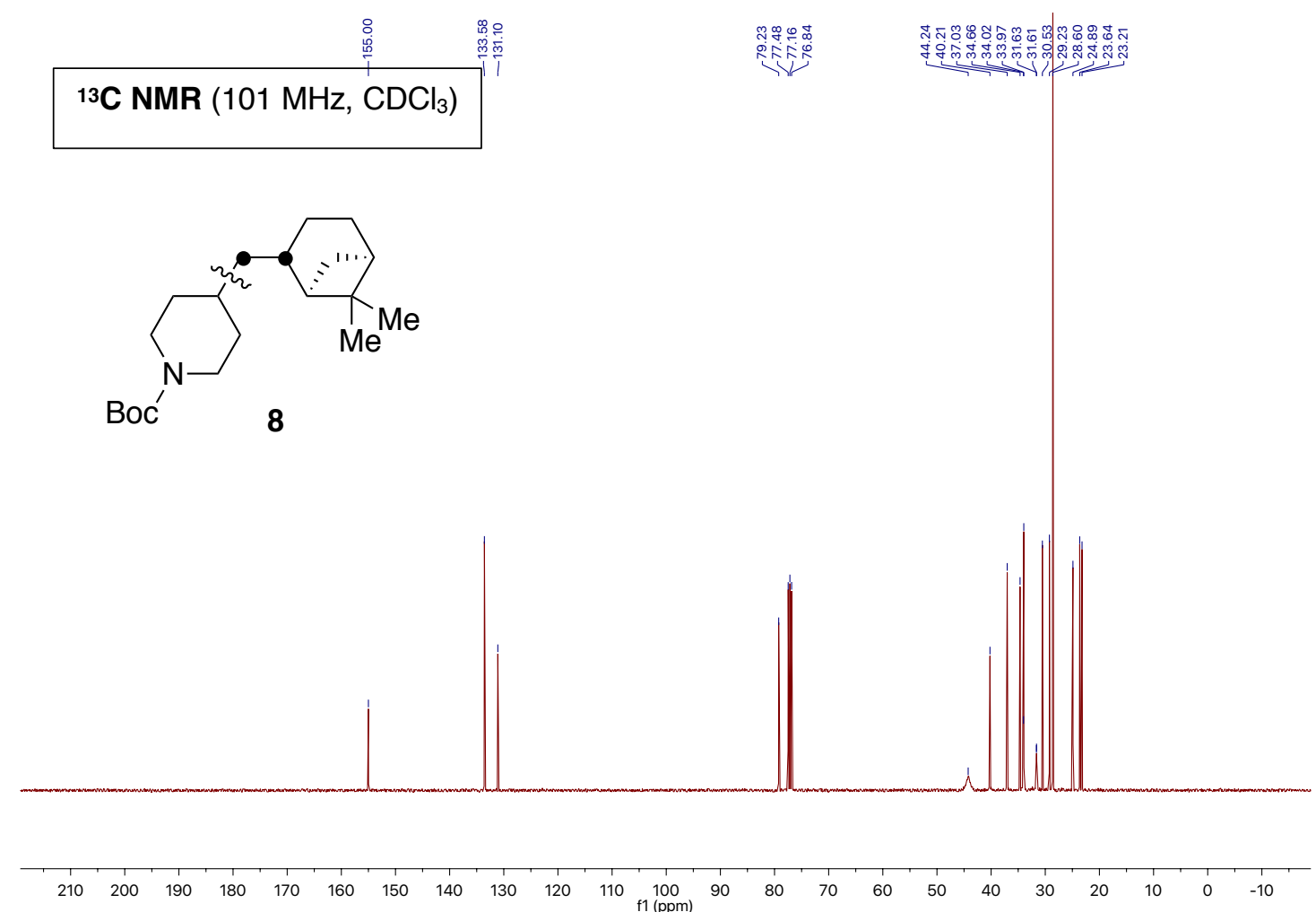

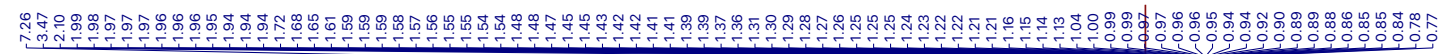

1H NMR (400 MHz, $\left.\mathrm{CDCl}_{3}\right)$<smiles></smiles>

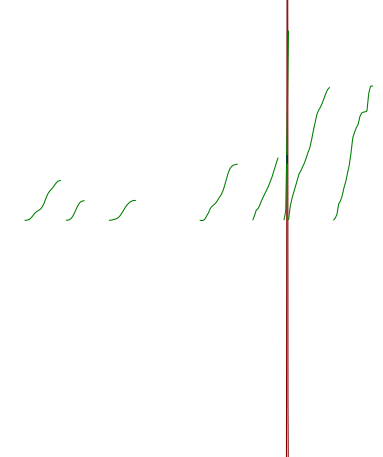

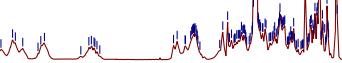

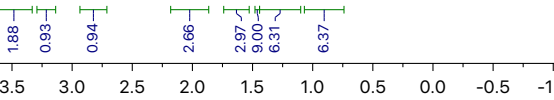


${ }^{13} \mathrm{C} \mathrm{NMR}\left(101 \mathrm{MHz}, \mathrm{CDCl}_{3}\right)$

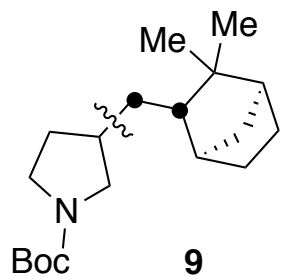<smiles>CC(C)(C)OC1C(COCCCCCCC2CCN(C(C)(C)C)CC2)C2OC(C)(C)OC2C2OC(C)(C)OC12</smiles>
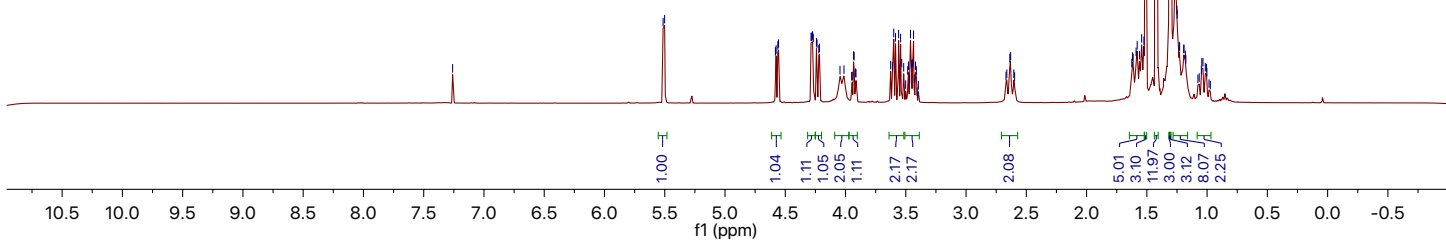


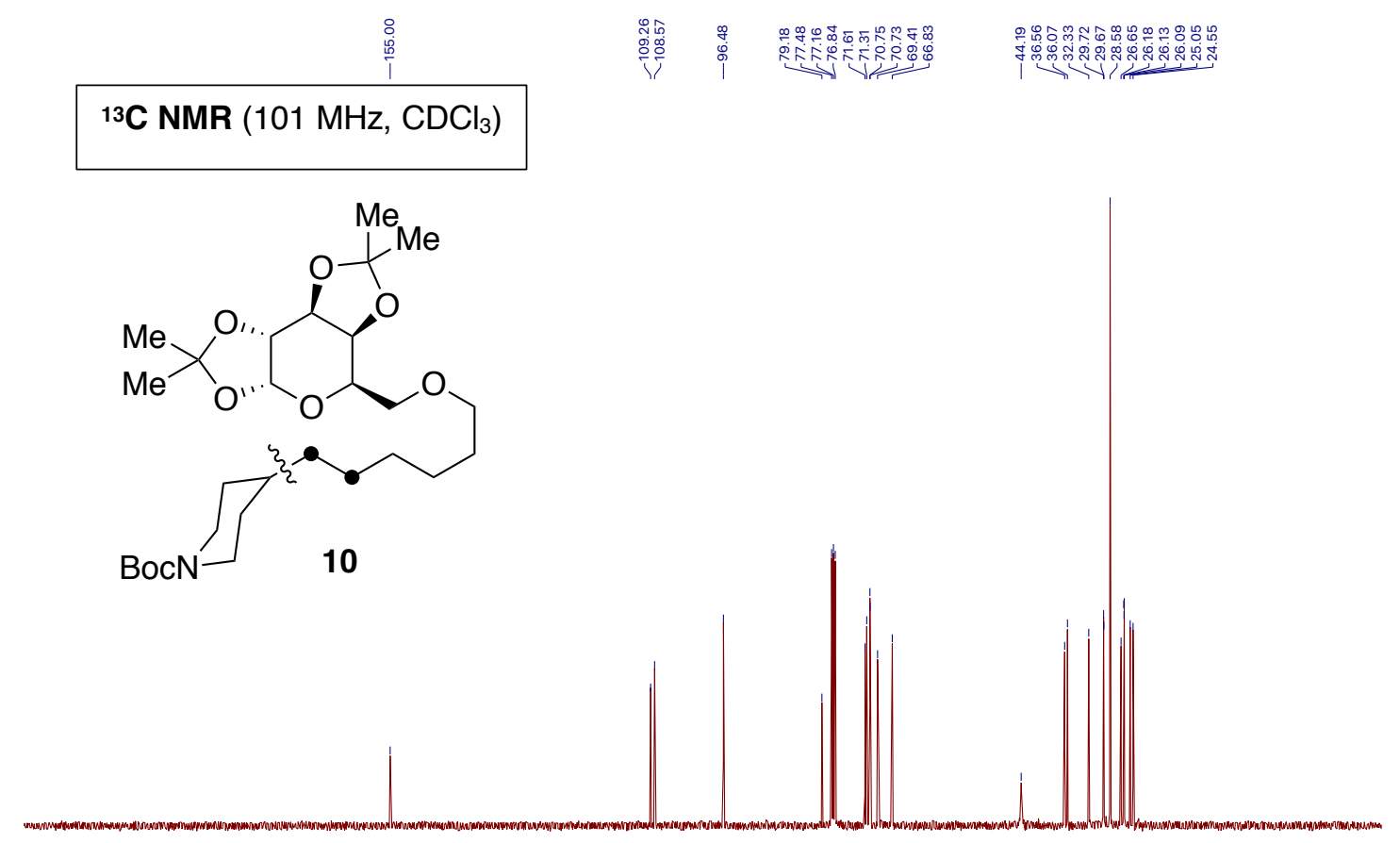

$\begin{array}{lllllllllllllllllllllll}210 & 200 & 190 & 180 & 170 & 160 & 150 & 140 & 130 & 120 & 110 & 100 & 90 & 80 & 70 & 60 & 50 & 40 & 30 & 20 & 10 & 0 & -10\end{array}$

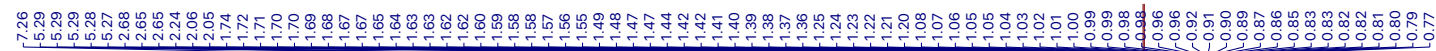

1H NMR (400 MHz, $\left.\mathrm{CDCl}_{3}\right)$

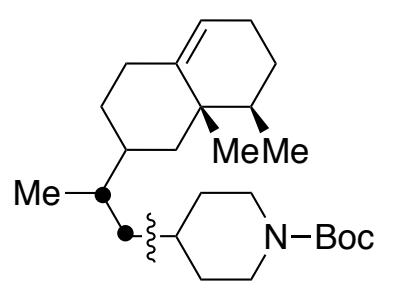

11

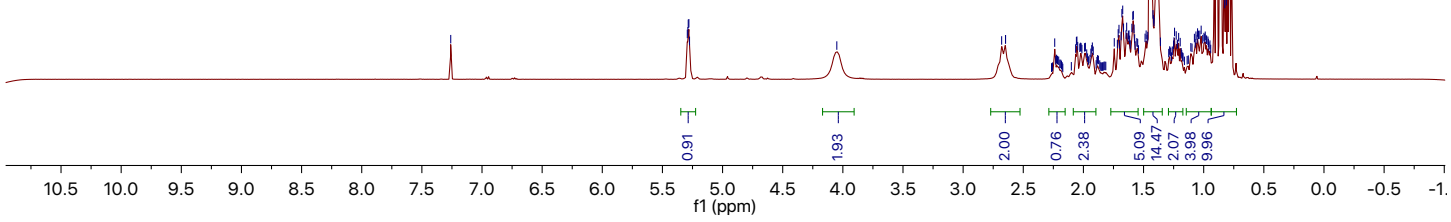


${ }^{13} \mathrm{C}$ NMR $\left(101 \mathrm{MHz}, \mathrm{CDCl}_{3}\right)$

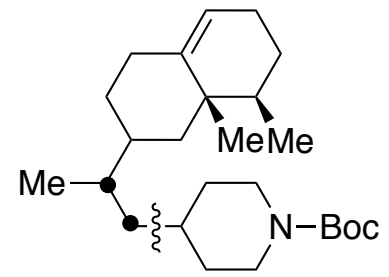

11

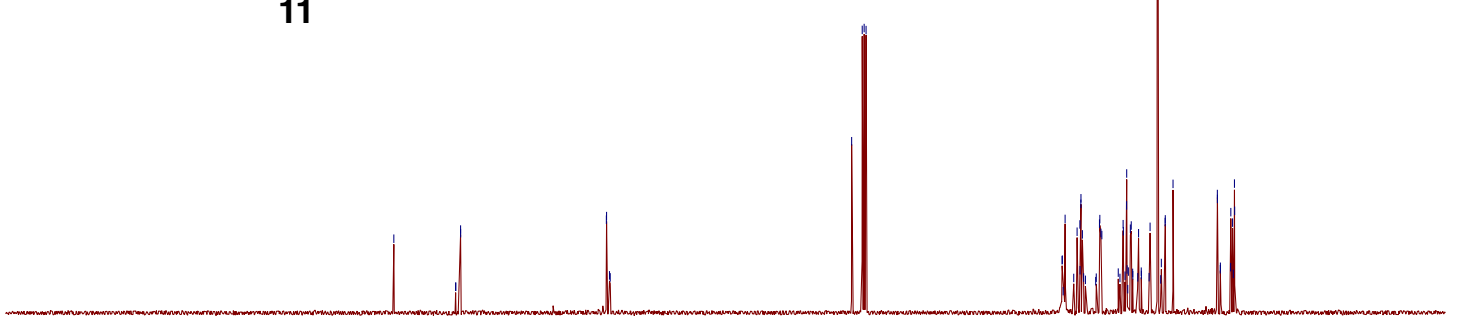

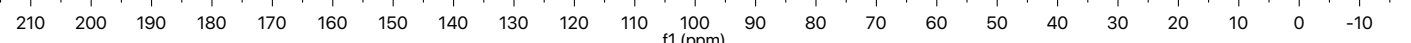

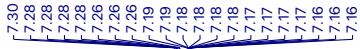

i.

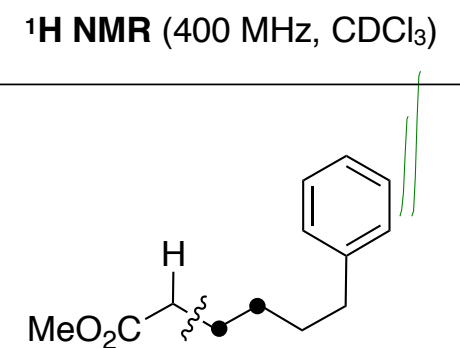

12
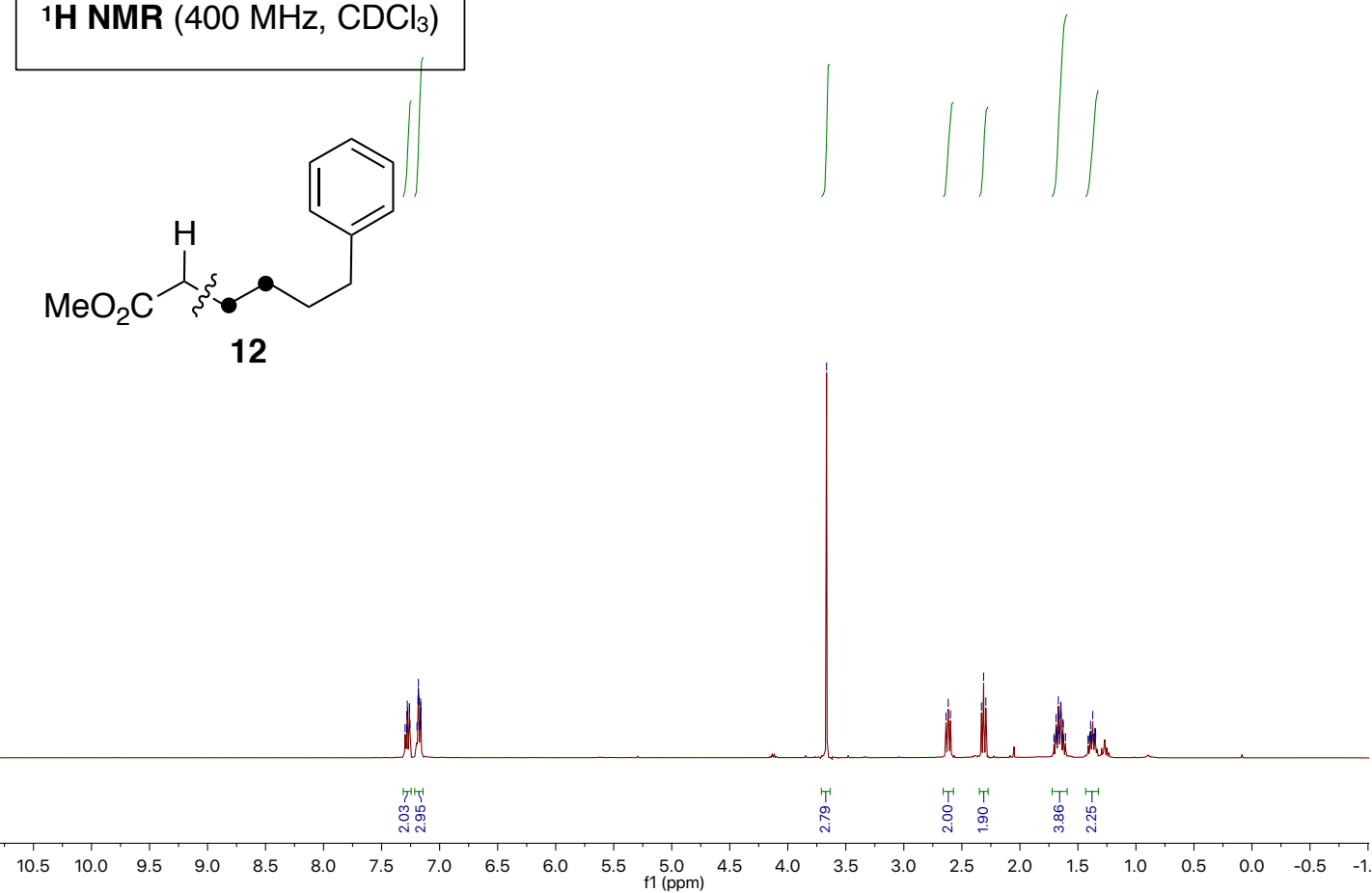

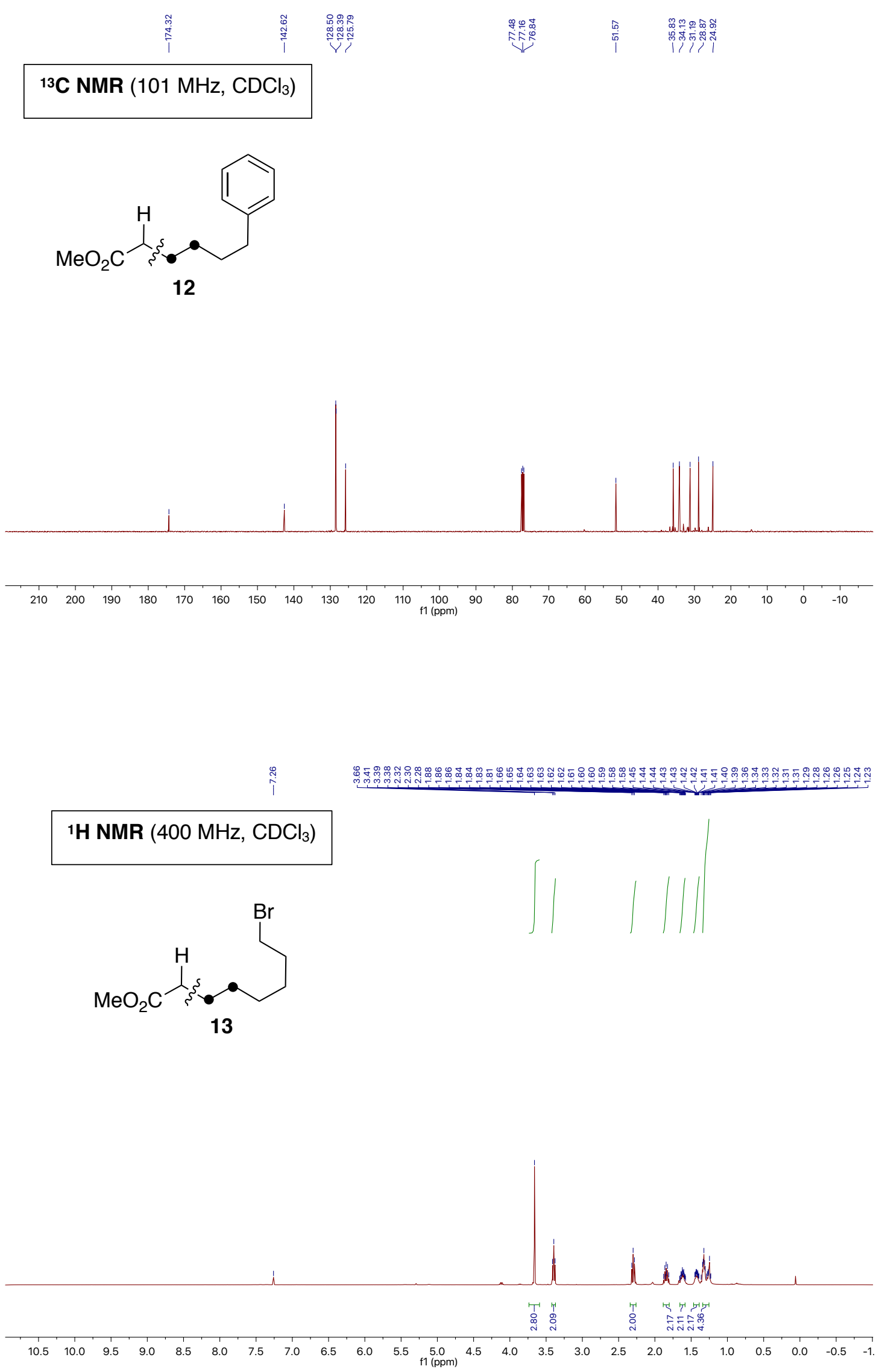


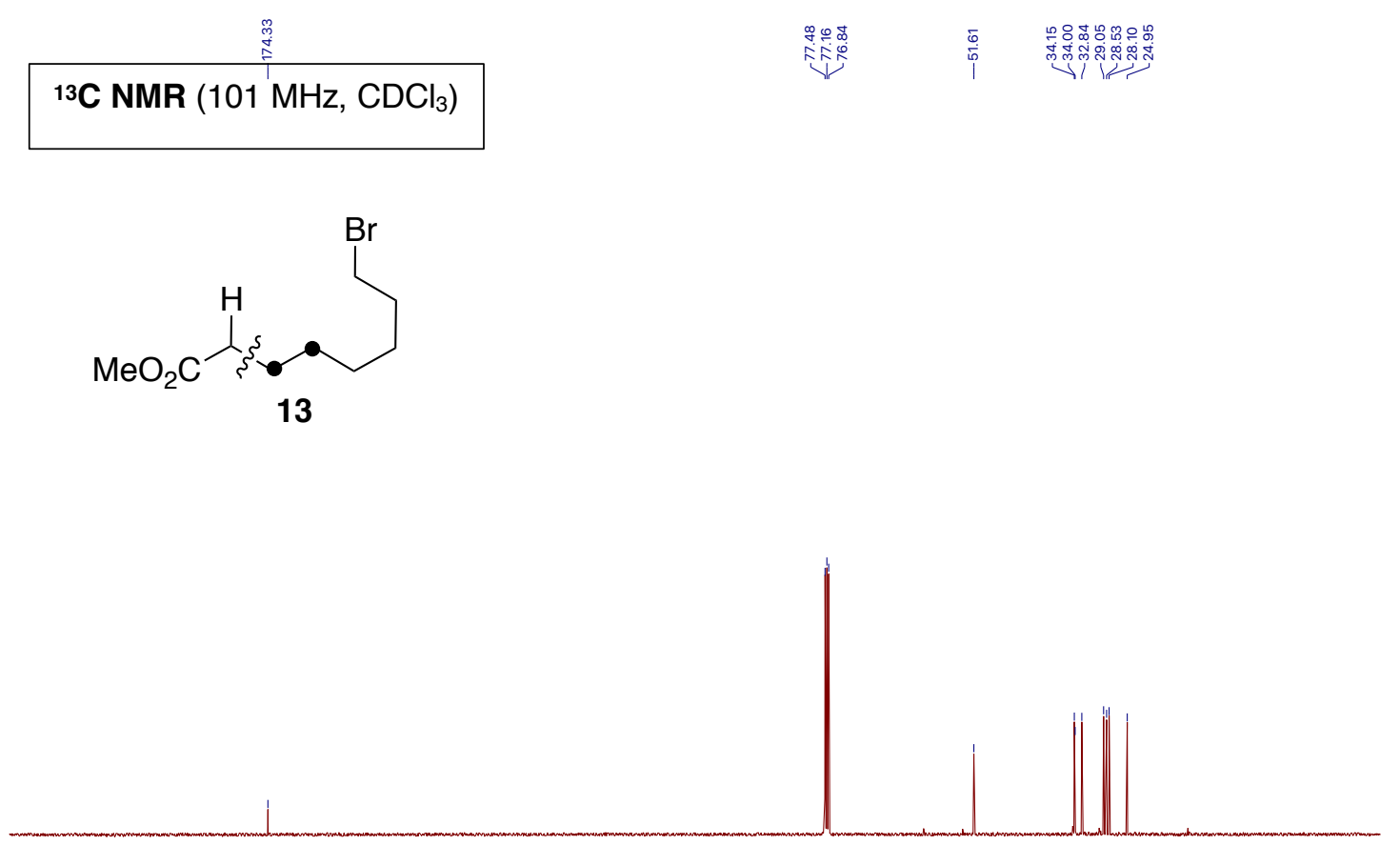

$\begin{array}{lllllllllllllllllllllllll}210 & 200 & 190 & 180 & 170 & 160 & 150 & 140 & 130 & 120 & 110 & \begin{array}{c}100 \\ \mathrm{f} 1(\mathrm{ppm})\end{array} & 90 & 80 & 70 & 60 & 50 & 40 & 30 & 20 & 10 & 0 & -10\end{array}$

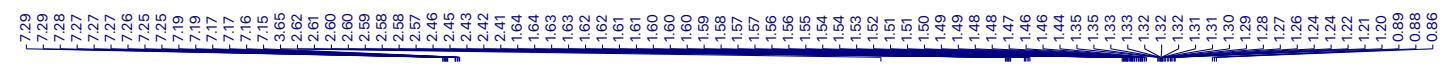

1H NMR (400 MHz, $\left.\mathrm{CDCl}_{3}\right)$<smiles>COC(=O)[C](CC(=[W])CCc1ccccc1)CC(C)C</smiles>

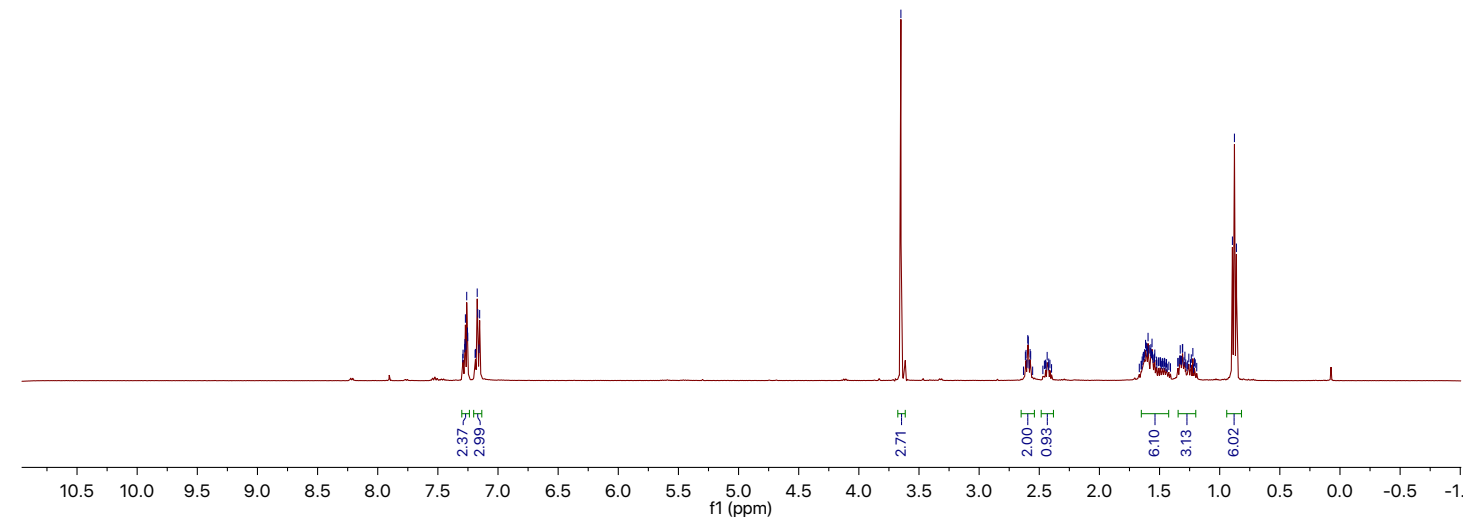




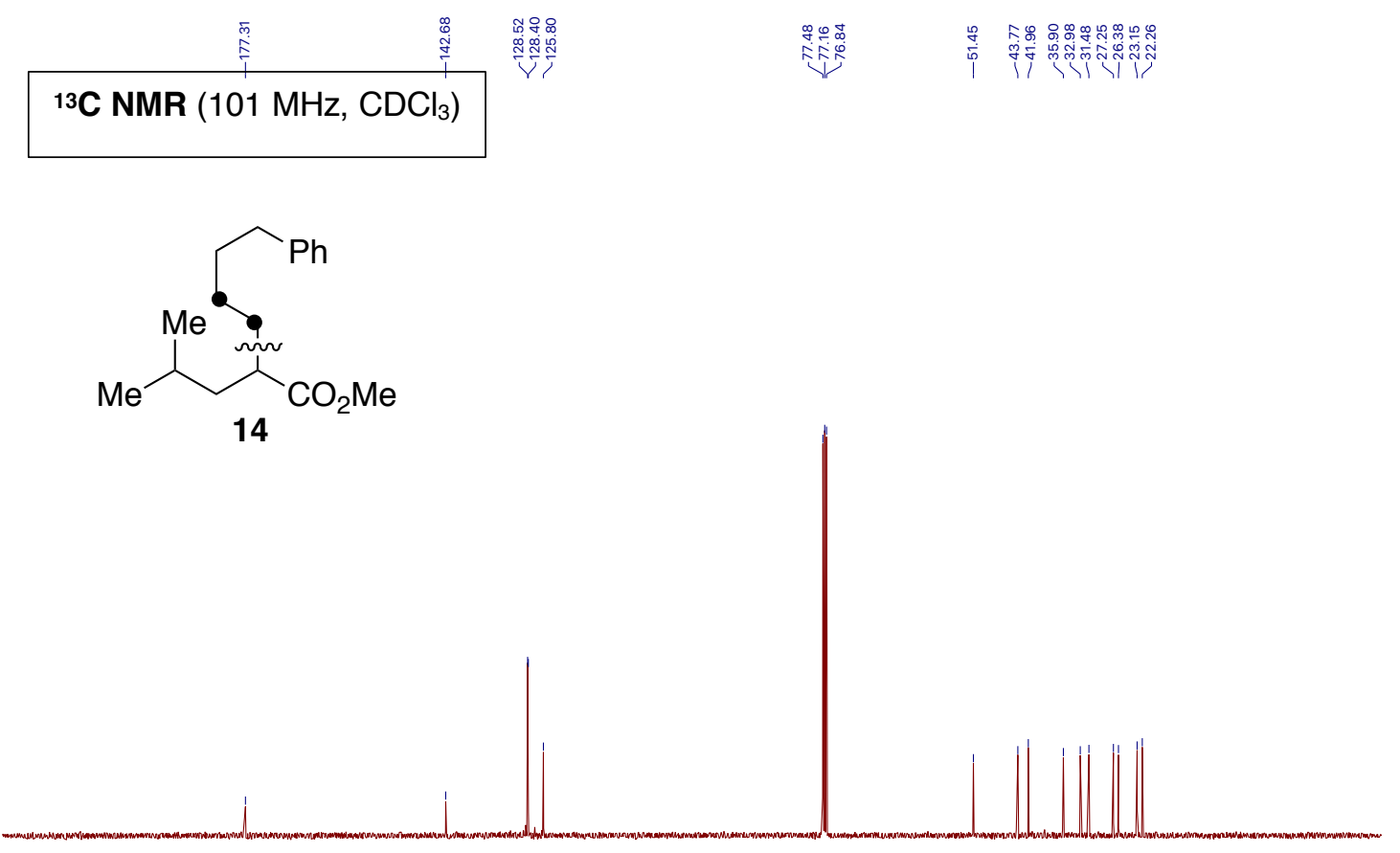

$\begin{array}{lllllllllllllllllllllllllllllllll}210 & 200 & 190 & 180 & 170 & 160 & 150 & 140 & 130 & 120 & 110 & 100 & 90 & 80 & 70 & 60 & 50 & 40 & 30 & 20 & 10 & 0 & -10\end{array}$

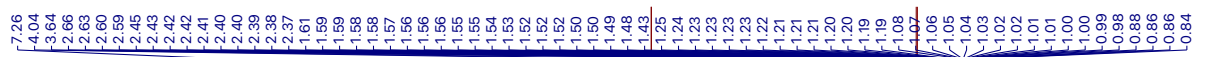

1H NMR (400 MHz, $\left.\mathrm{CDCl}_{3}\right)$
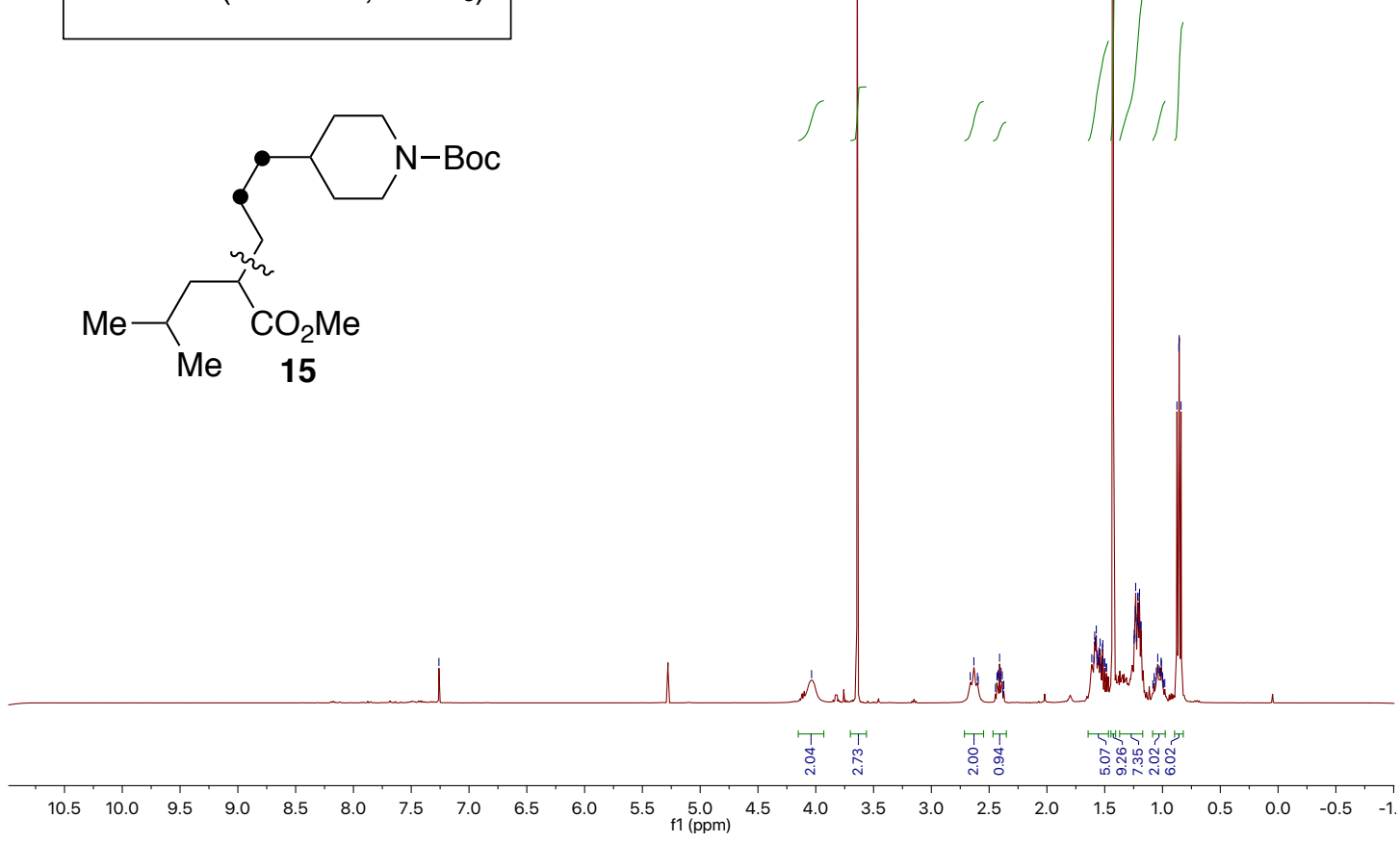
${ }^{13} \mathrm{C} \mathrm{NMR}\left(101 \mathrm{MHz}, \mathrm{CDCl}_{3}\right)$
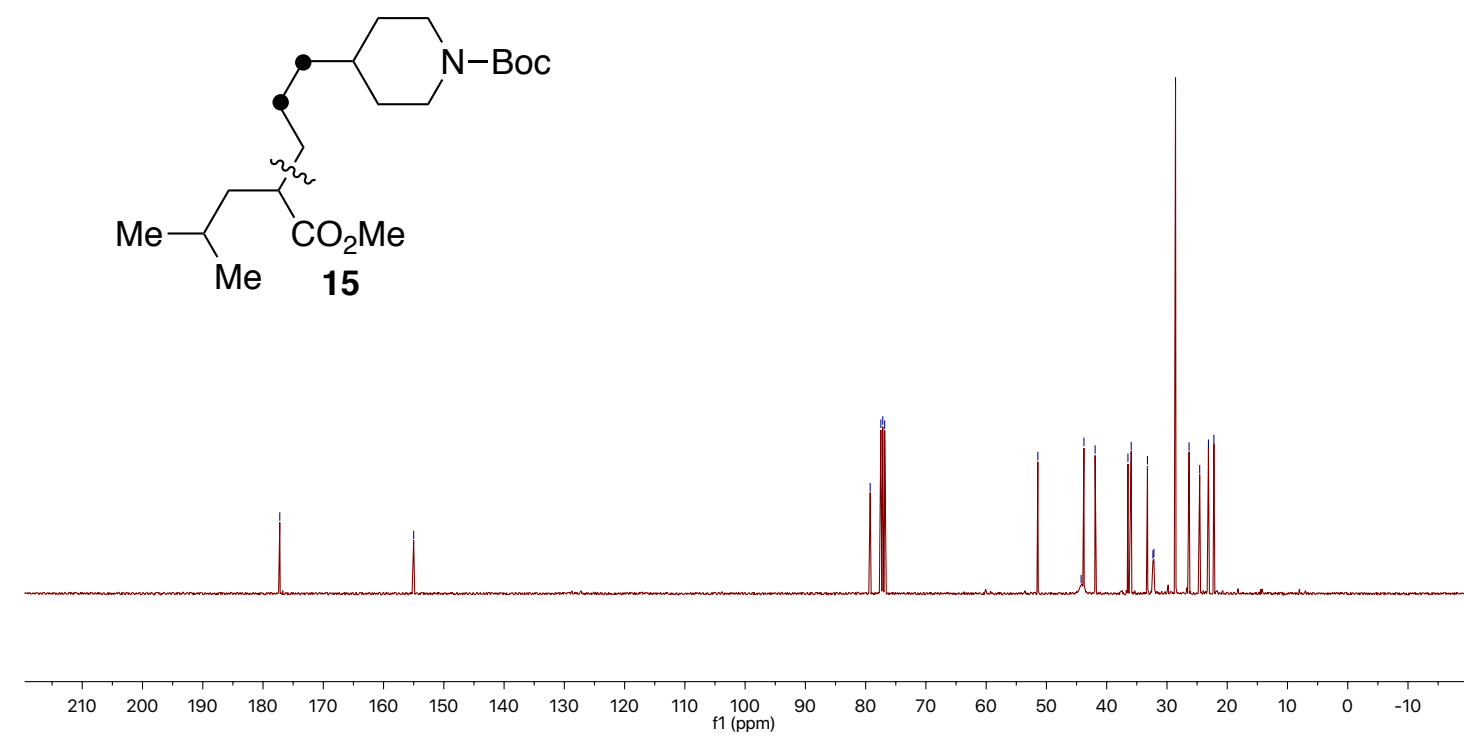

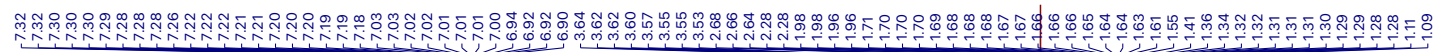

\section{H NMR $\left(400 \mathrm{MHz}, \mathrm{CDCl}_{3}\right)$}<smiles>Cc1cccc(C)c1OCC(C)CCCCc1ccccc1</smiles>

16

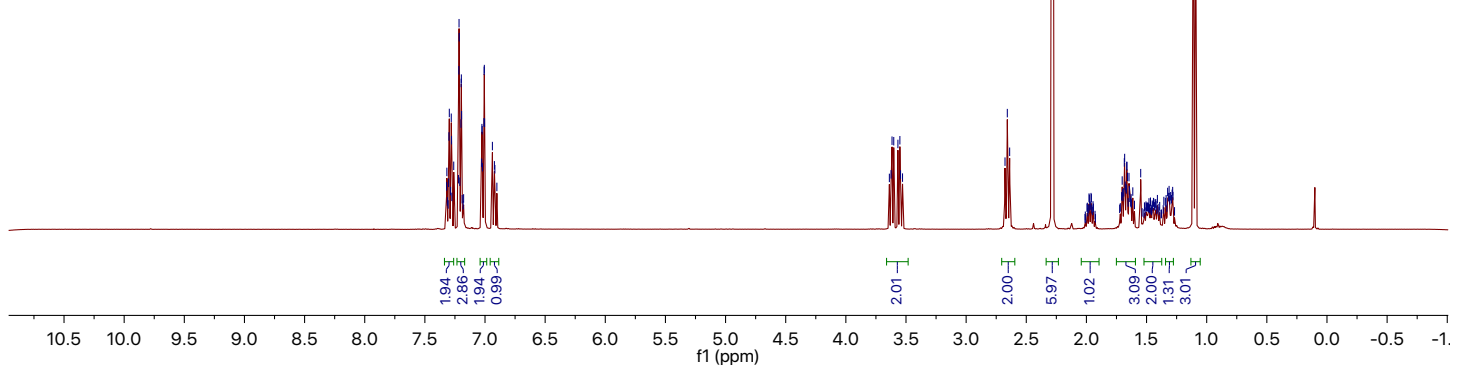




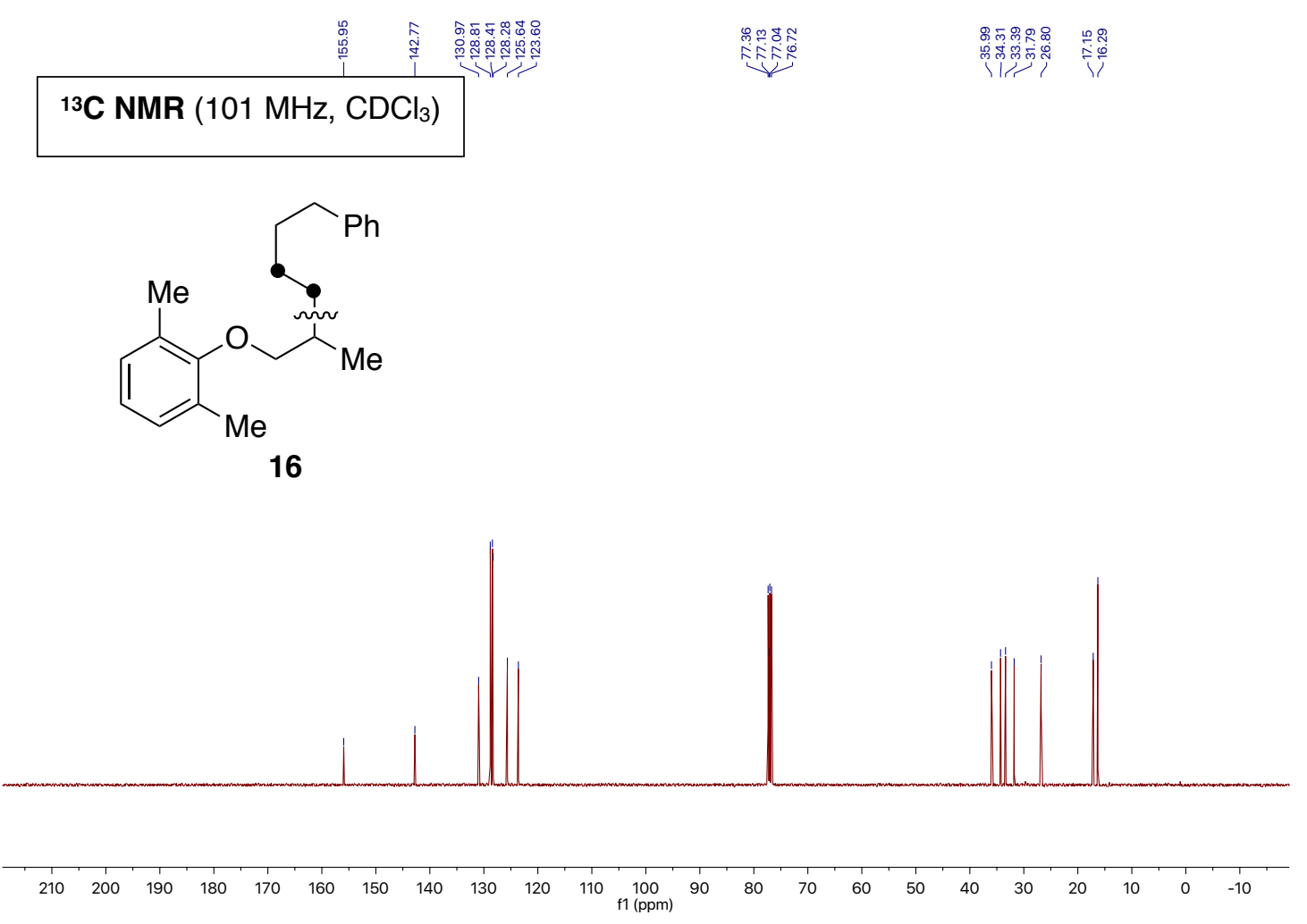

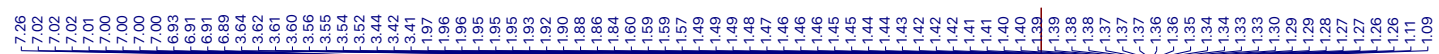
1H NMR (400 MHz, $\left.\mathrm{CDCl}_{3}\right)$<smiles>Cc1cccc(C)c1OCC(C)CCCCCCBr</smiles><smiles>CCCC[I-]I</smiles> 


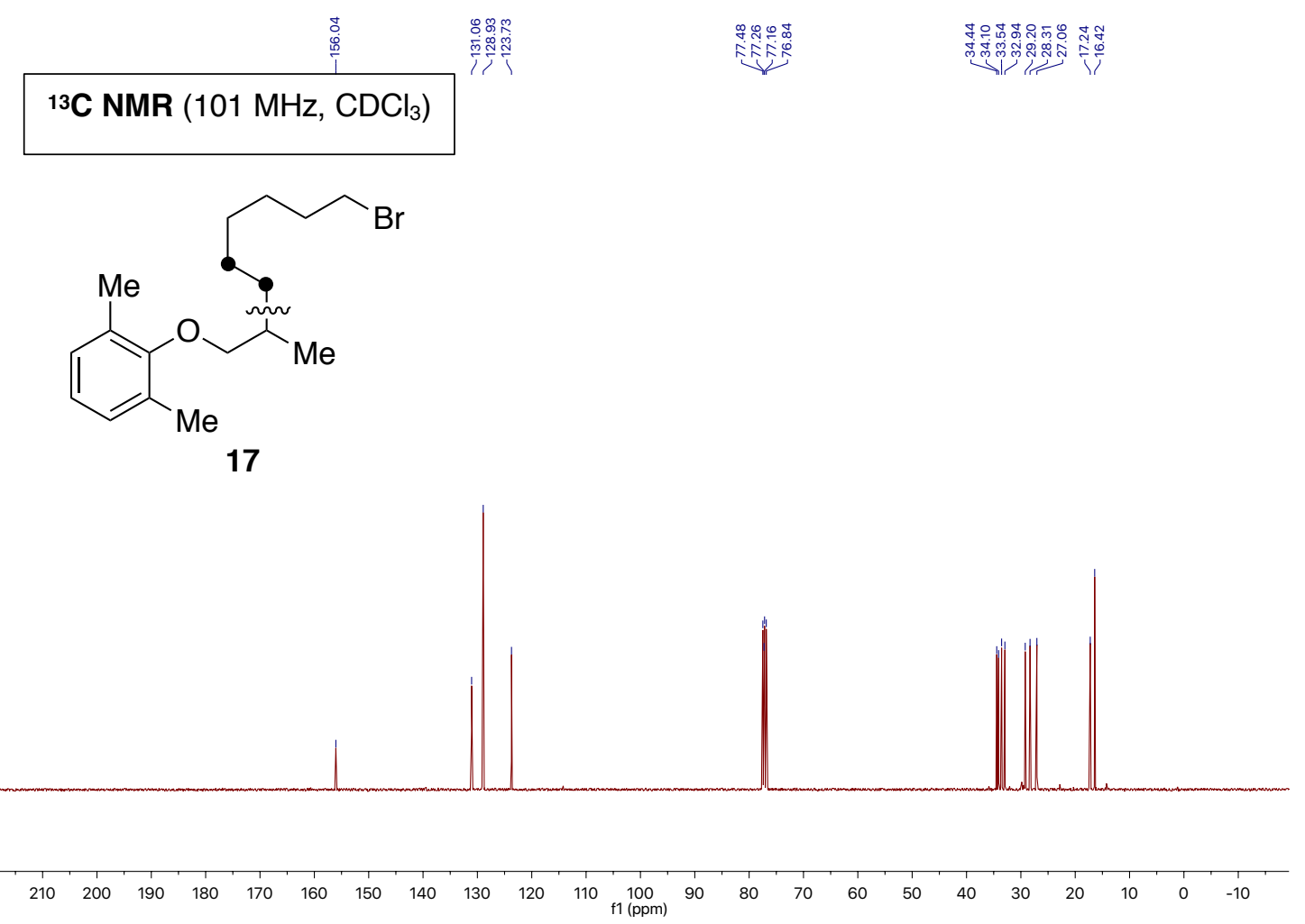

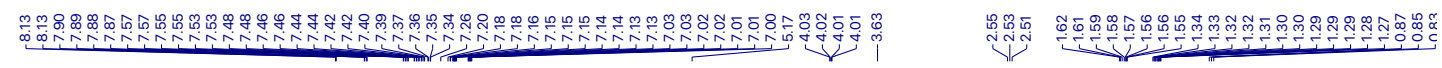

1H NMR (400 MHz, $\left.\mathrm{CDCl}_{3}\right)$<smiles>CCC(CCCc1cccc(Cl)c1)[C@H](CC)OC(=O)Cc1ccc2c(c1)C(=O)c1ccccc1CO2</smiles>
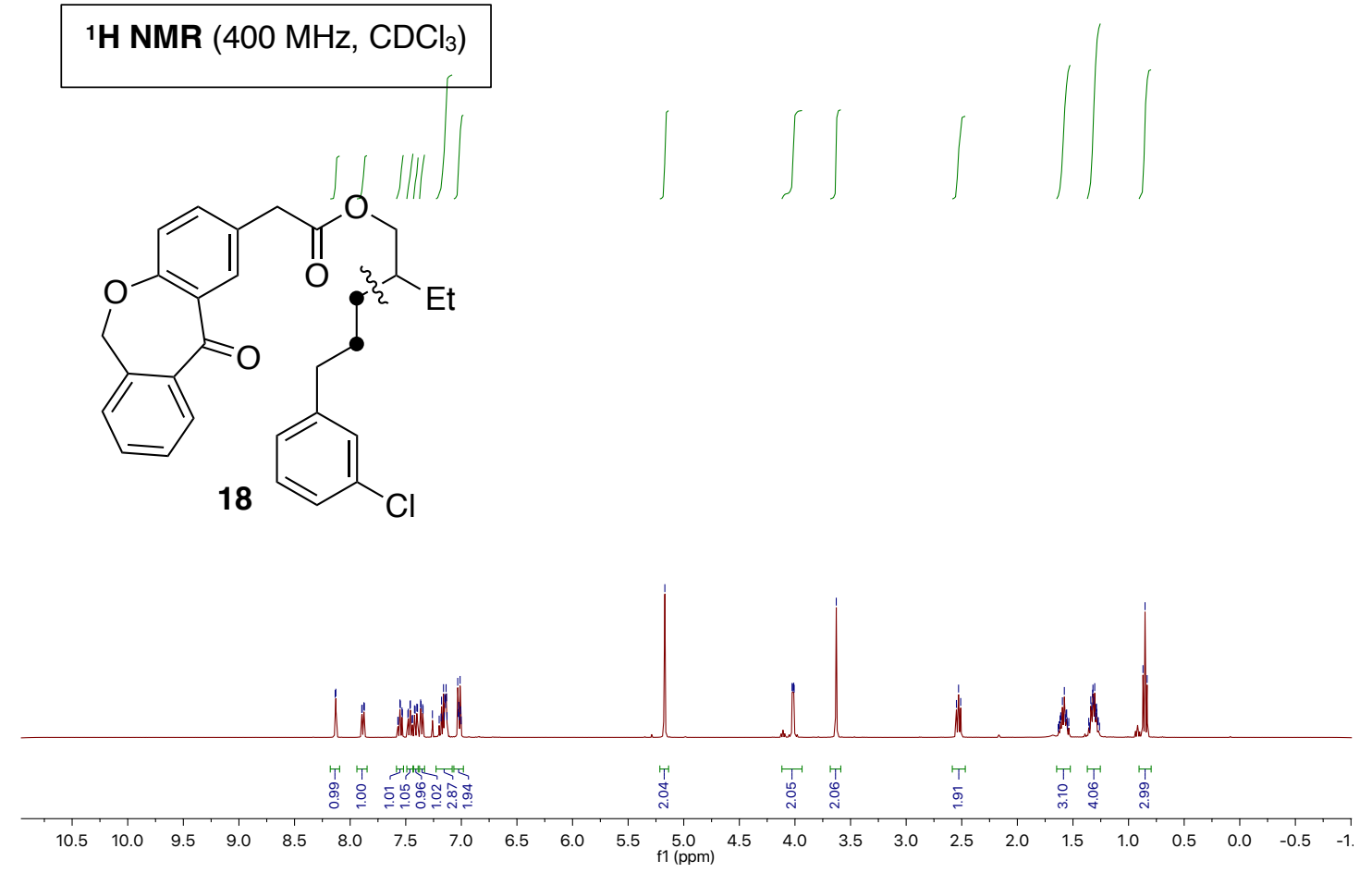
${ }^{13} \mathrm{C}$ NMR (101 MHz, $\left.\mathrm{CDCl}_{3}\right)$<smiles></smiles>

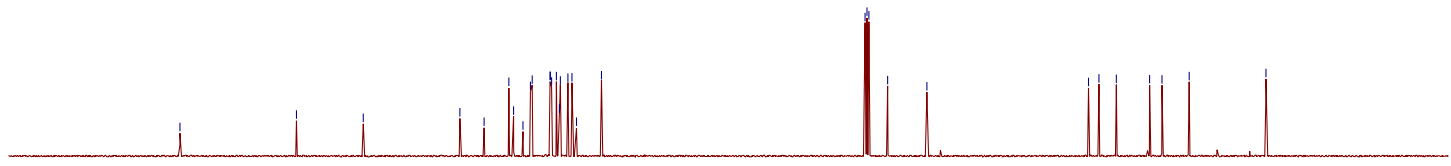

$\begin{array}{llllllllllllllllllllllllllllllllll}210 & 200 & 190 & 180 & 170 & 160 & 150 & 140 & 130 & 120 & 110 & 100 & 90 & 80 & 70 & 60 & 50 & 40 & 30 & 20 & 10 & 0 & -10\end{array}$

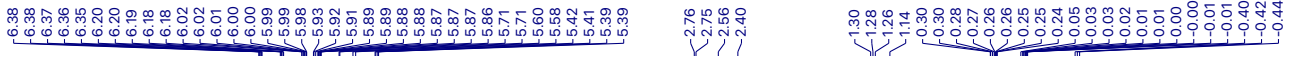

1H NMR (400 MHz, $\left.\mathrm{CDCl}_{3}\right)$<smiles>C#C[C@H](CC)[C@H](CC)CCCc1ccccc1</smiles>

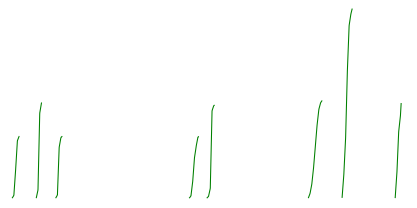

19

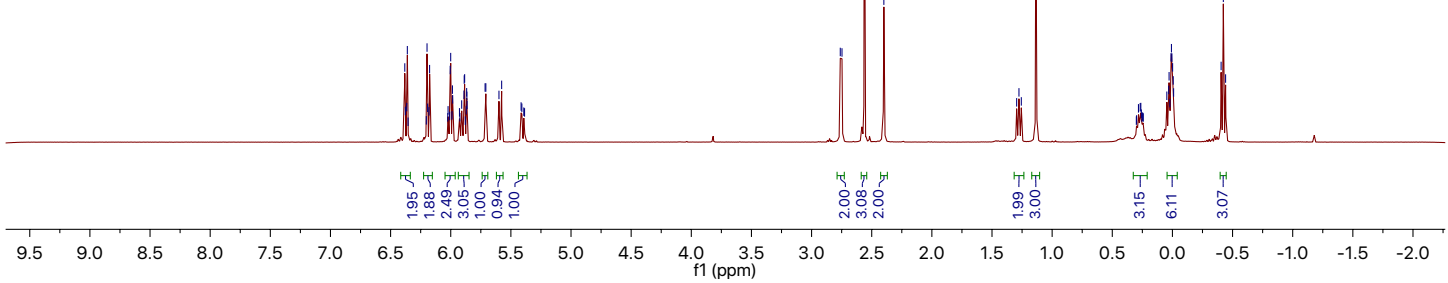


${ }^{13} \mathrm{C} \mathrm{NMR}\left(101 \mathrm{MHz}, \mathrm{CDCl}_{3}\right)$

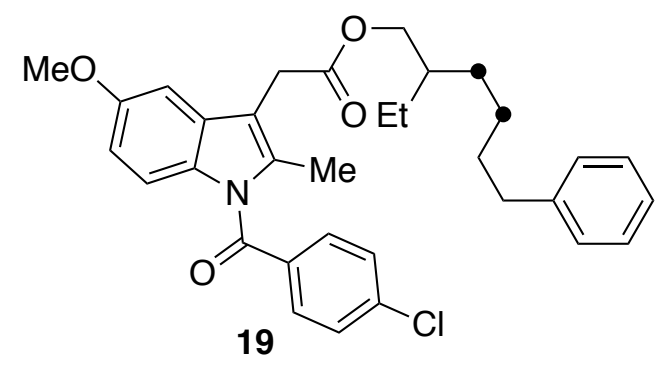

\begin{tabular}{llllllllllllllllllllllllllllllllllllll}
\hline 30 & 220 & 210 & 200 & 190 & 180 & 170 & 160 & 150 & 140 & 130 & 120 & 110 & 100 & 90 & 80 & 70 & 60 & 50 & 40 & 30 & 20 & 10 & 0 & -10 & -20
\end{tabular}

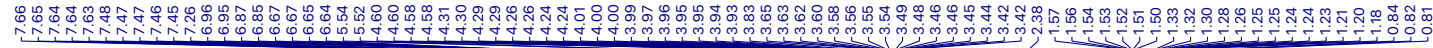

1H NMR (400 MHz, $\left.\mathrm{CDCl}_{3}\right)$<smiles>CCC(CCCCCCCOC[C@H]1O[C@@H]2OC(C)(C)O[C@@H]2[C@H]2OC(C)(C)O[C@@H]21)COC(=O)Cc1c(C)n(C(=O)c2ccc(Cl)cc2)c2ccc(OC)cc12</smiles>
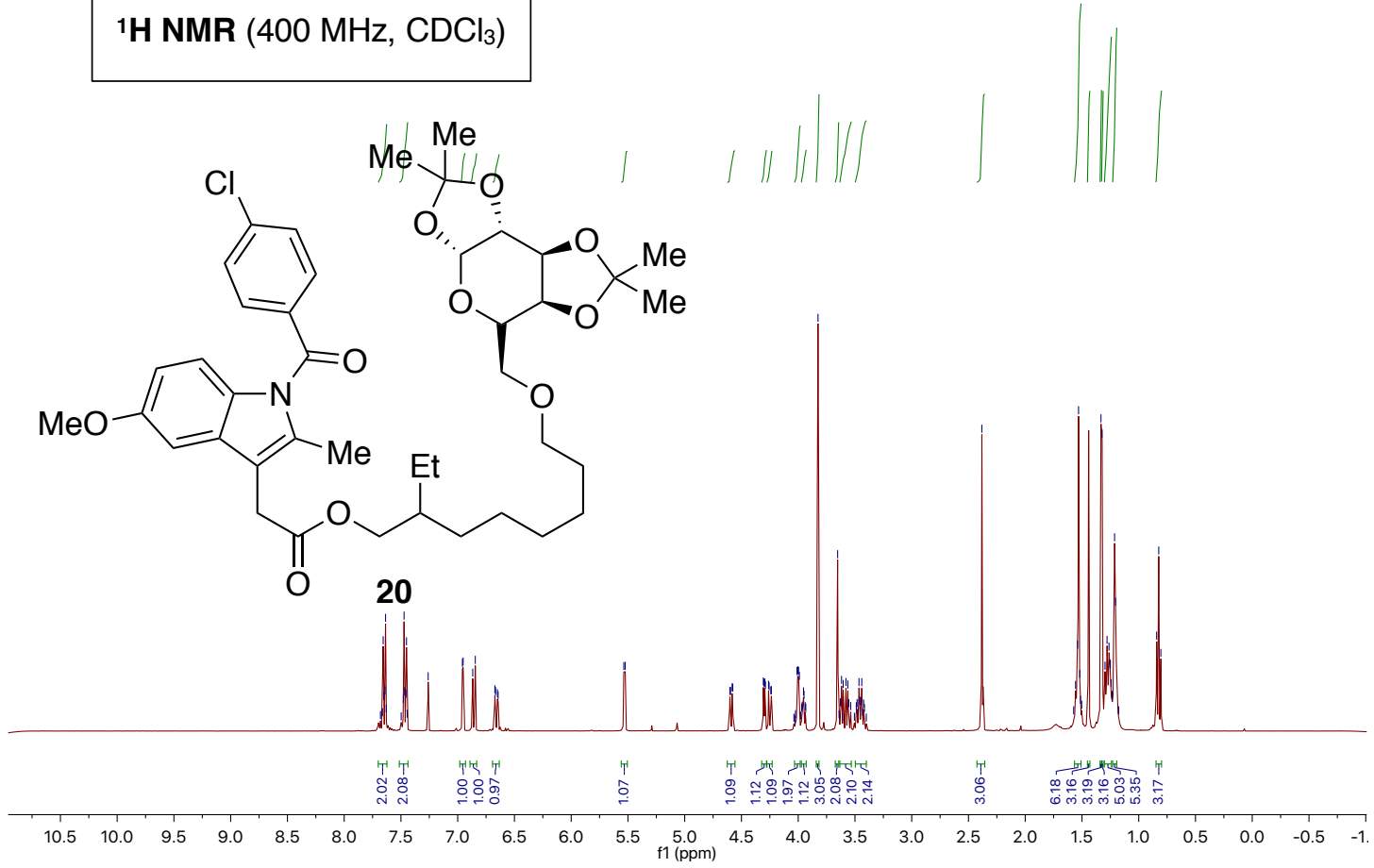


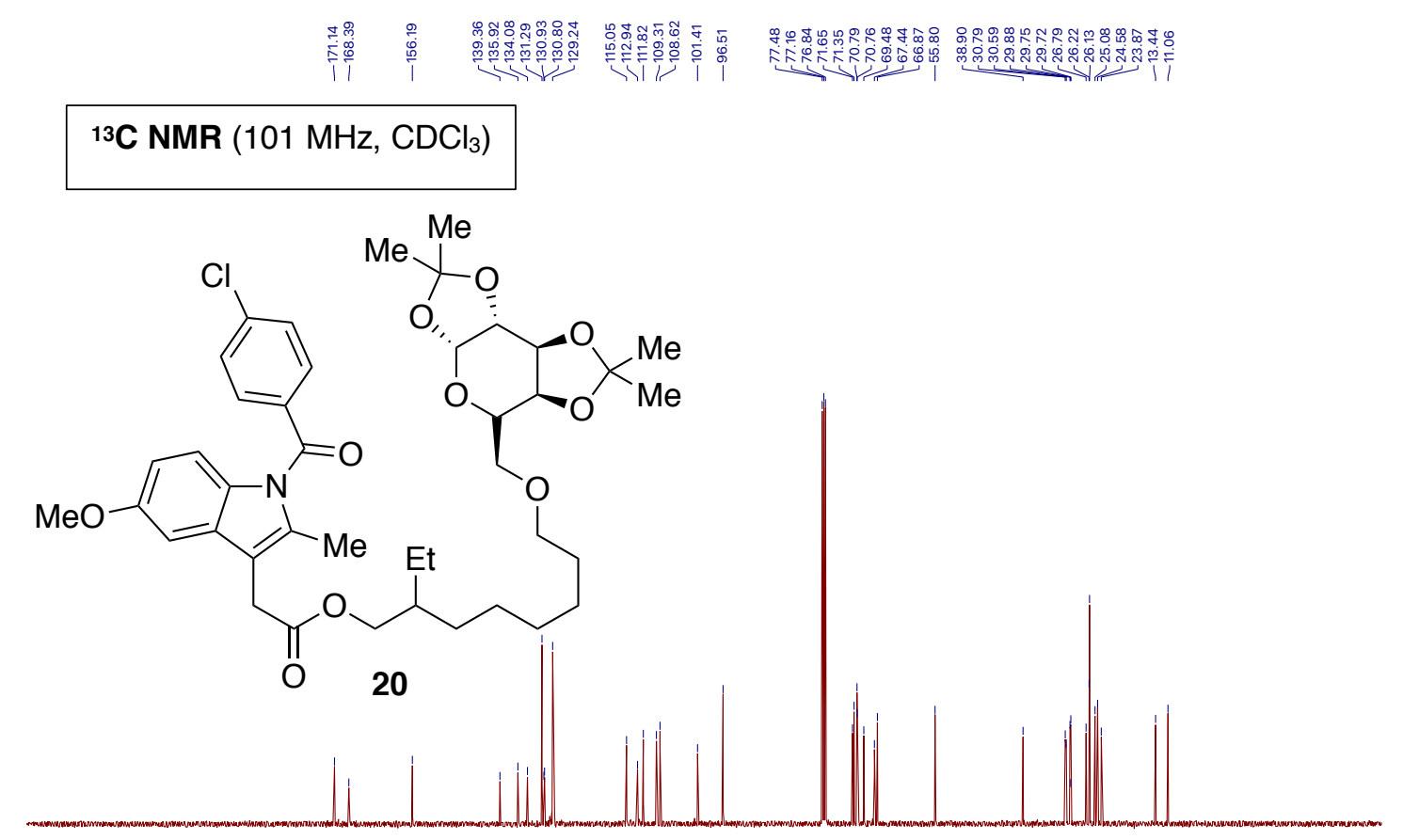

\begin{tabular}{lllllllllllllllllllllllllllllllllllll}
\hline 30 & 220 & 210 & 200 & 190 & 180 & 170 & 160 & 150 & 140 & 130 & 120 & 110 & 100 & 90 & 80 & 70 & 60 & 50 & 40 & 30 & 20 & 10 & 0 & -10 & -20
\end{tabular}

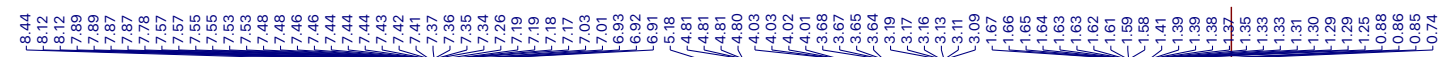

'1H NMR $\left(400 \mathrm{MHz}, \mathrm{CDCl}_{3}\right)$

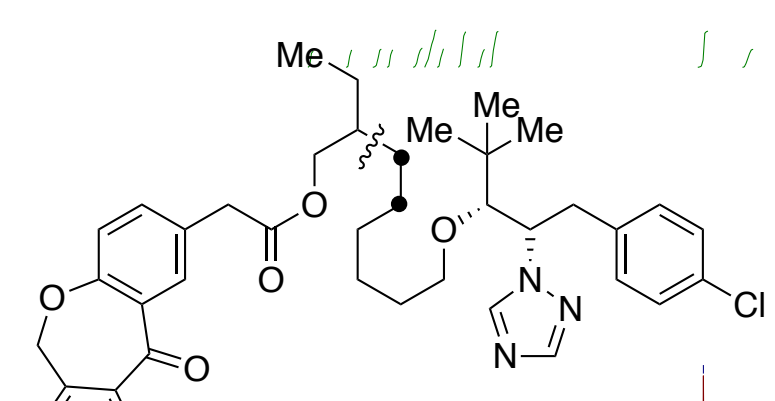

21

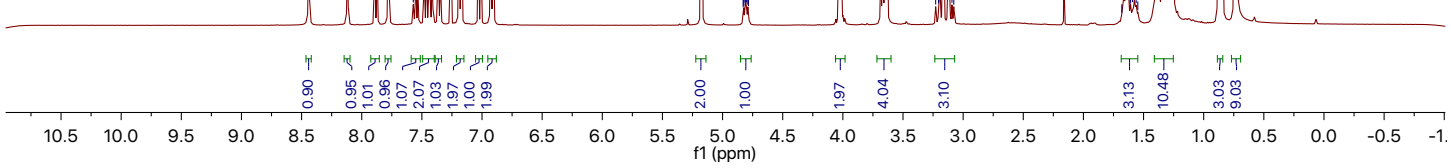




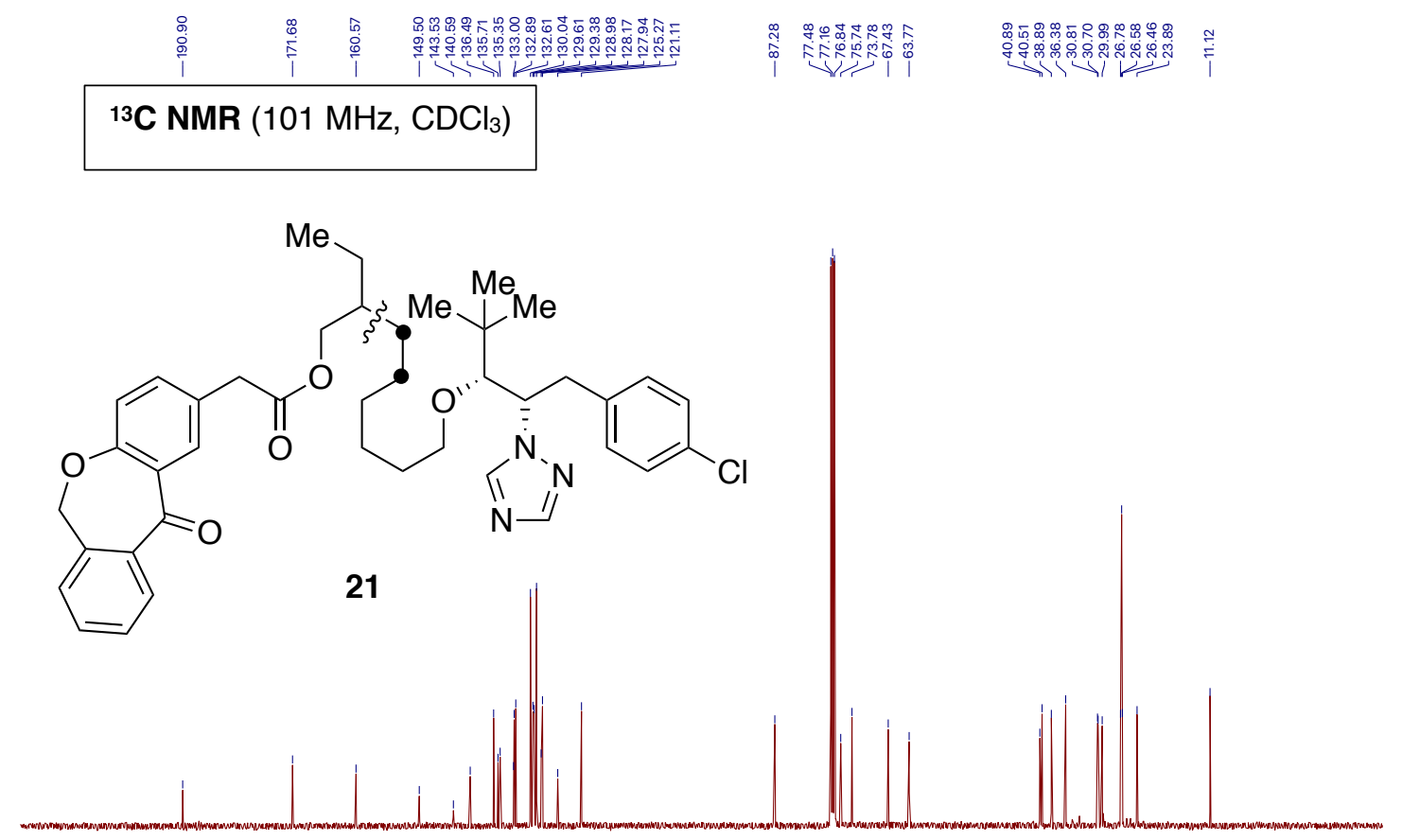

$\begin{array}{llllllllllllllllllllllllllllllllllll}210 & 200 & 190 & 180 & 170 & 160 & 150 & 140 & 130 & 120 & 110 & 100 & 90 & 80 & 70 & 60 & 50 & 40 & 30 & 20 & 10 & 0 & -10\end{array}$

1H NMR (400 MHz, $\left.\mathrm{CDCl}_{3}\right)$

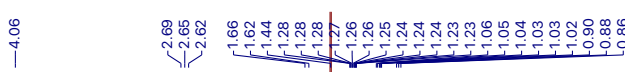<smiles>CCC1CCN(C(=O)OC(C)(C)C)CC1</smiles>

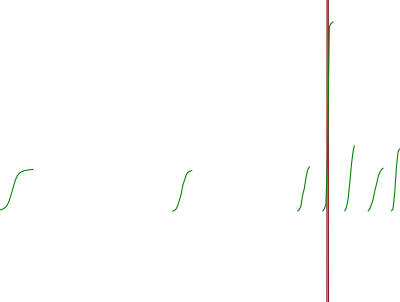




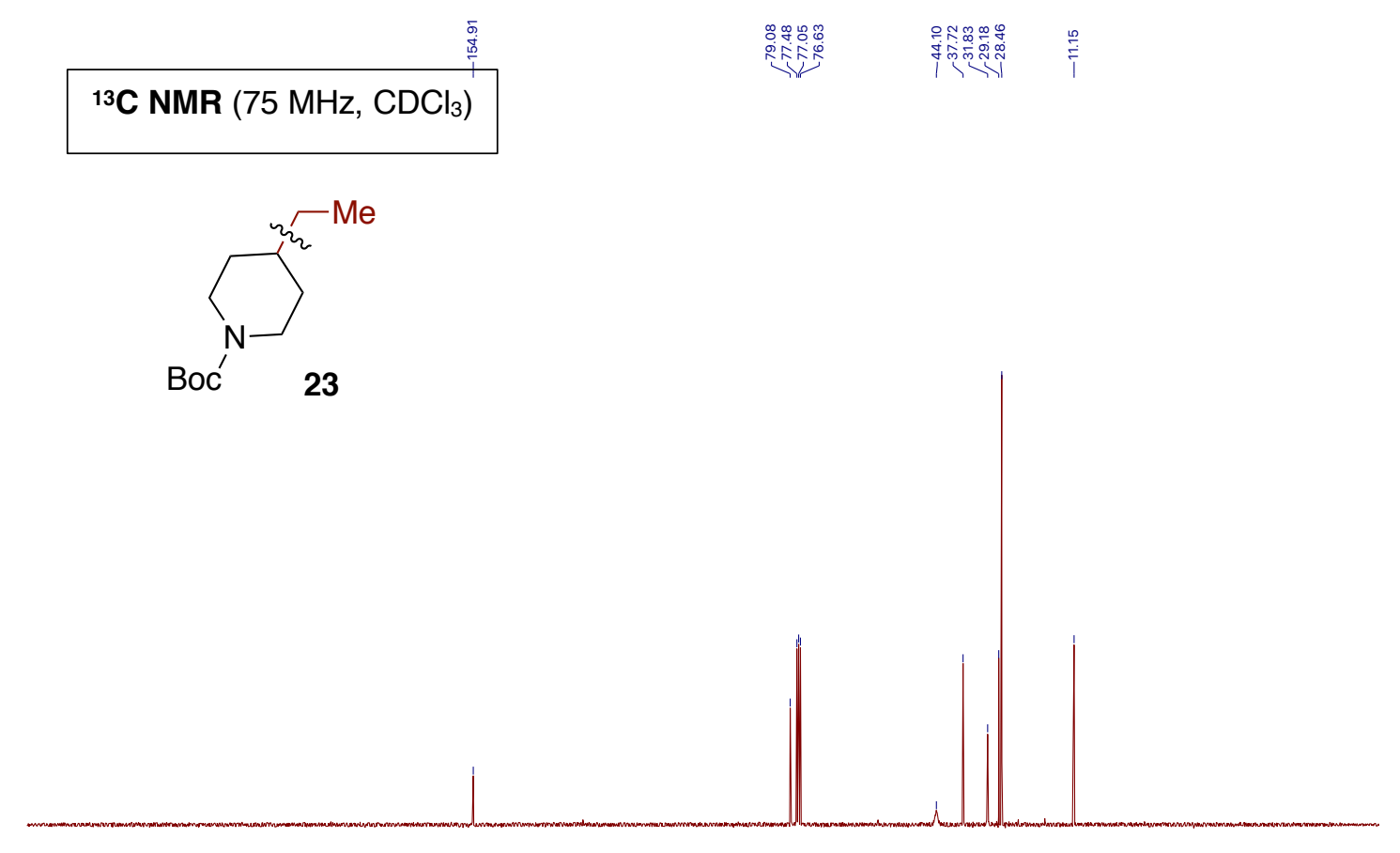

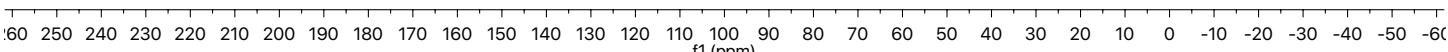

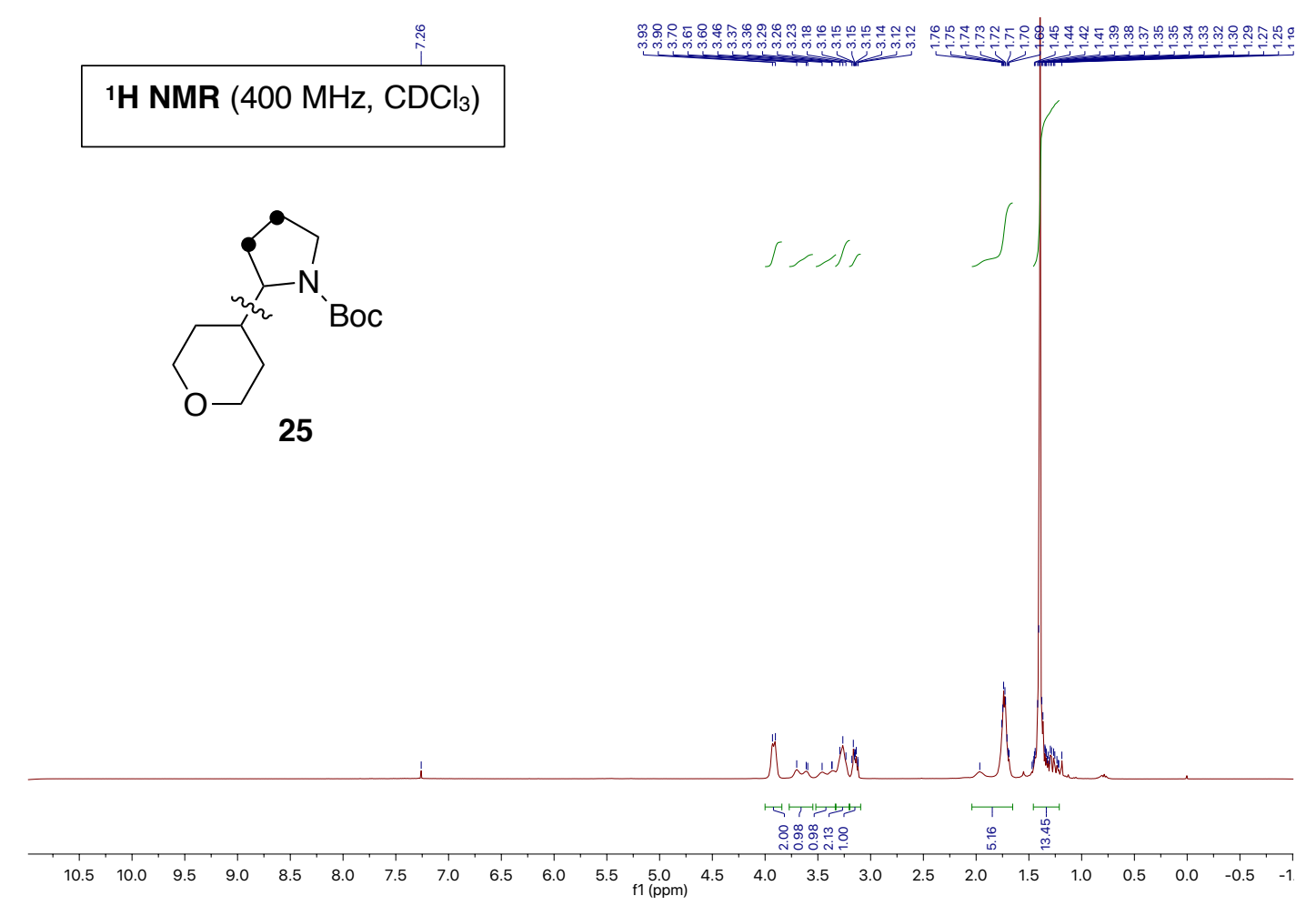


$\frac{2}{2}$

${ }^{13} \mathrm{C} \mathrm{NMR}\left(101 \mathrm{MHz}, \mathrm{CDCl}_{3}\right)$

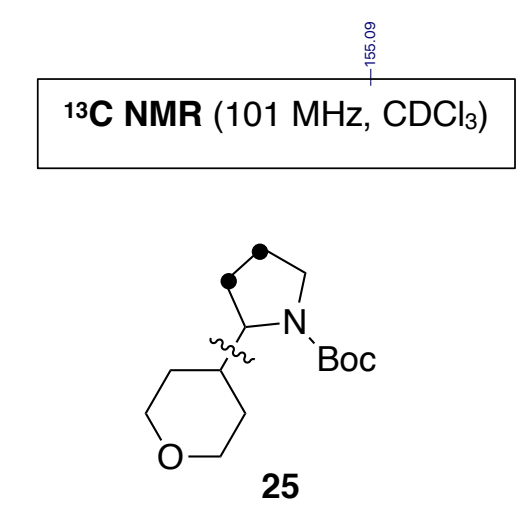

ail wil

$\begin{array}{lllllllllllllllllllllllll}210 & 200 & 190 & 180 & 170 & 160 & 150 & 140 & 130 & 120 & 110 & 100 & 90 & 80 & 70 & 60 & 50 & 40 & 30 & 20 & 10 & 0 & -10\end{array}$

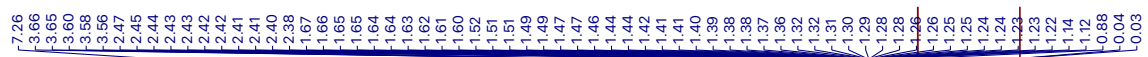

1H NMR (400 MHz, $\left.\mathrm{CDCl}_{3}\right)$

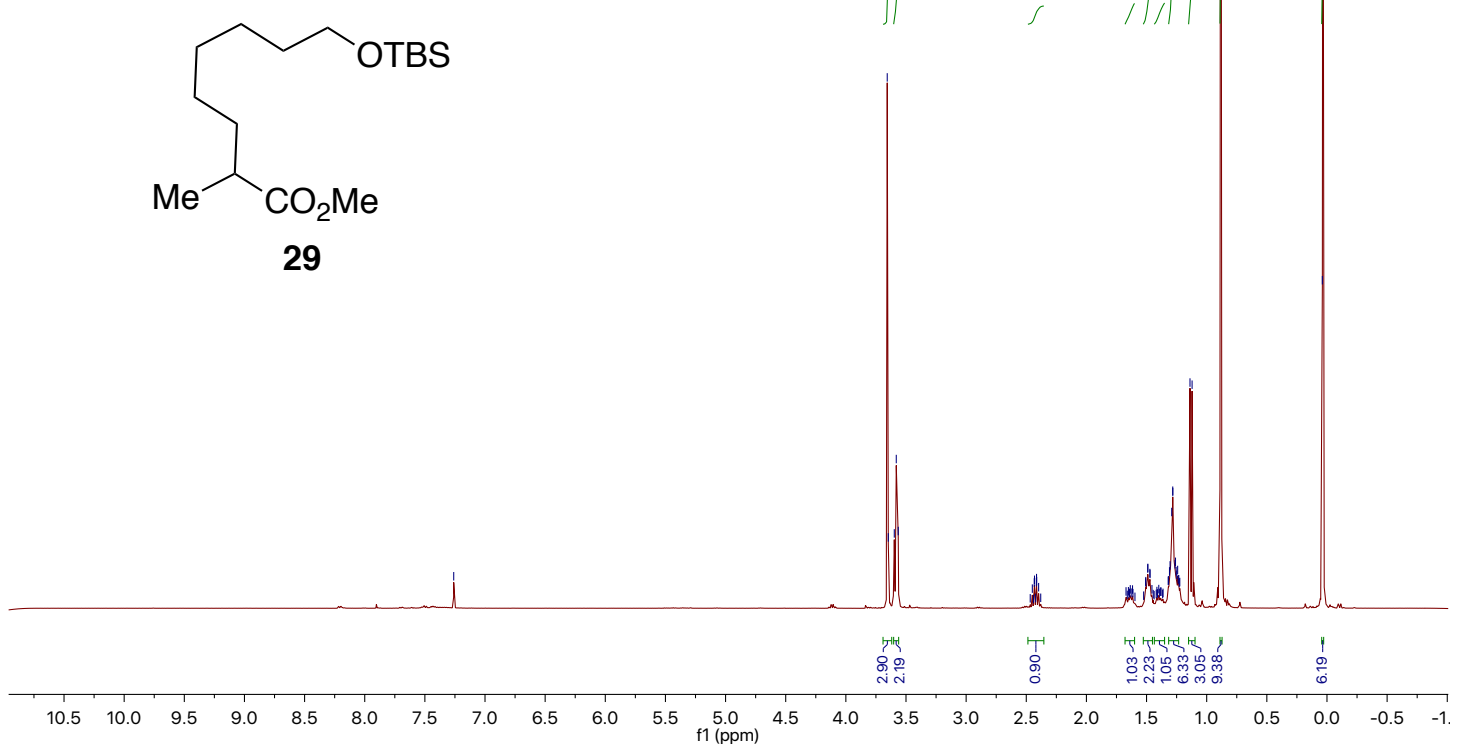




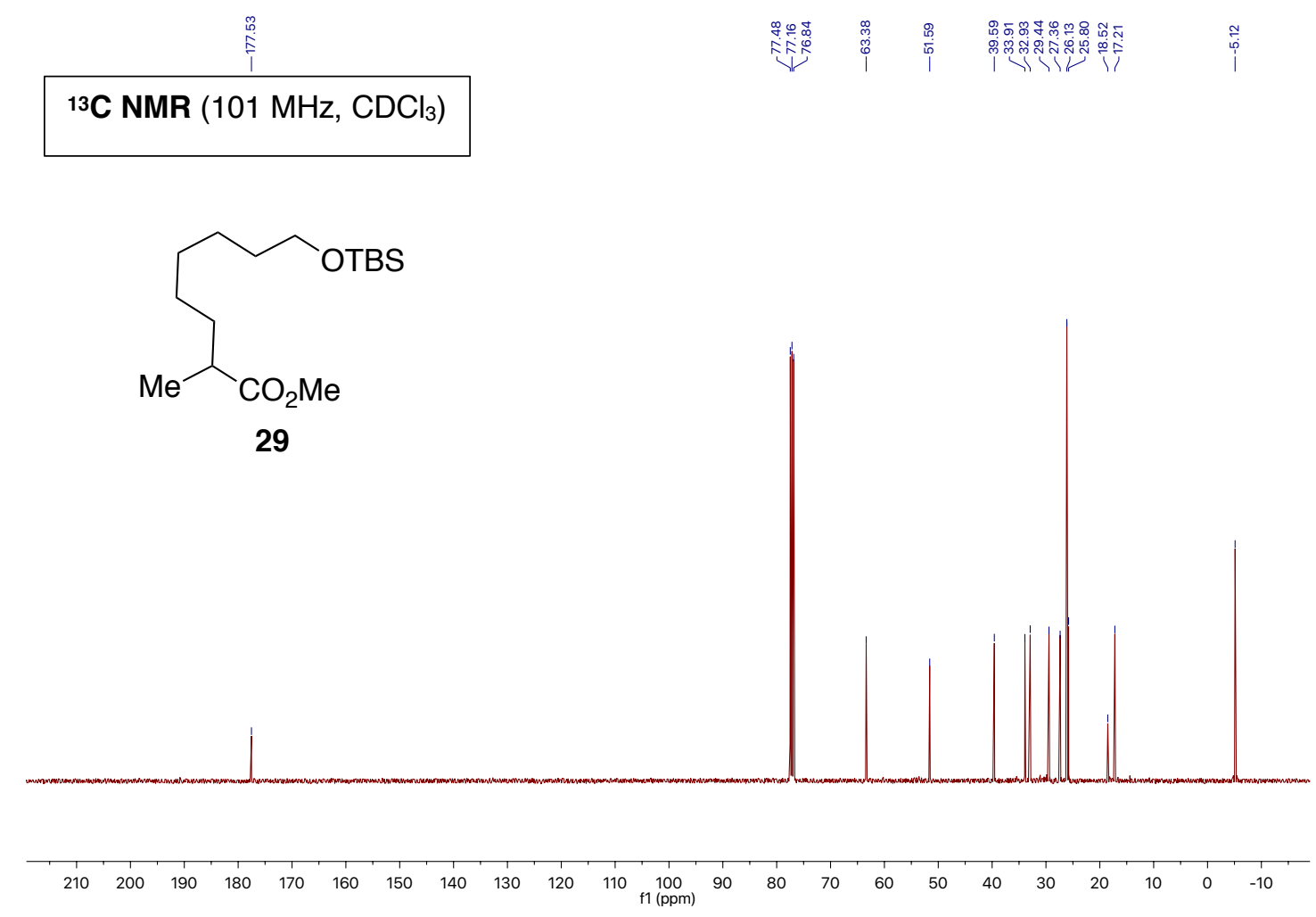

